Precision Measurement of the Lifetime and Decay Asymmetry of the Cascade-Zero Baryon

\begin{abstract}
A THESIS
SUBMITTED TO THE FACULTY OF THE GRADUATE SCHOOL OF THE UNIVERSITY OF MINNESOTA
\end{abstract}

BY

Keith Alan Thorne

IN PARTIAL FULFILLMENT OF THE REQUIREMENTS

FOR THE DEGREE OF

DOCTOR OF PHILOSOPHY

April, 1990 
(c) Keith Alan Thorne 1990 


\title{
Precision Measurement of the Lifetime and Decay Asymmetry of the Cascade-Zero Baryon
}

\author{
by Keith Alan Thorne \\ Under the supervision of Professor Kenneth J. Heller
}

\begin{abstract}
The non-leptonic decays of strange baryons such as the cascade-zero $\left(\Xi^{0}\right)$ are a sensitive probe of long-range strong interaction effects on electro-weak interaction physics. Recent improvements in the accuracy of the theoretical predictions of the decay amplitudes will soon require improved precision in the experimental measurements of the decay rates and asymmetry parameters. In an experiment at Fermilab, a multi-wire chamber magnetic spectrometer and a lead glass electromagnetic calorimeter were used to reconstruct the $\Lambda \rightarrow p \pi^{-}$and $\Xi^{0} \rightarrow \Lambda \pi^{0}$ hyperon decays in a high-energy neutral beam. The measured lifetime of the $\Lambda$, based on 724,000 events, was $2.62 \pm 0.01$ (statistical + systematic error) $\times 10^{-10} \mathrm{~s}$. This data, together with previous results, demonstrated the Lorentz invariance of the $\Lambda$ lifetime for momentum from 1 to $400 \mathrm{GeV} / \mathrm{c}$. The lifetime of the $\Xi^{0}$, from 88,000 events, was found to be $2.98 \pm 0.04$ (stat.) \pm 0.02 (syst.) $\times 10^{-10} \mathrm{~s}$. This measurement had an uncertainty almost three times smaller than any previous determination. The same $\Xi^{0}$ data was used to measure a value for the decay asymmetry product $\alpha_{\Lambda} \alpha_{\Xi}$ of $-0.242 \pm 0.006$ (stat.) \pm 0.006 (syst.).
\end{abstract}




\section{Acknowledgements}

High-energy physics experiments are very much a collective effort, dependent of the work of many more people than those researchers directly involved with the experiment. Many people at Fermilab gave what are too often unheralded efforts to make this experiment a success. Roger Tokarek deserves special praise for his extraordinary efforts as our beamline physicist, making sure that everything got installed and kept operating properly. Thanks go to the beamline control operators for listening to all our complaints about the beam quality, the magnets, etc., and interceding on our behalf with the masters of the accelerators. ( I'm sure that the coffee cake I brought in on Sunday mornings had nothing to do with it. ) In addition, I wish to recognize the operators in the main control room who endeavored to keep the Tevatron operating smoothly despite the tinkerings of the accelerator physicists. I also thank the Computer Department for help with our data acquisition electronics and software and the computer operators for mounting all the tapes and printer paper for my analysis and watching over the aging Cyber computers. Thanks must also go to the U. S. Department of Energy for footing the bill for all this.

I could not have completed my doctoral research without the guidance and support of my advisor, Kenneth Heller. His willingness to treat me more as a colleague than as his student contributed greatly to my satisfaction with my graduate work. Tom Devlin deserves much credit for his continued encouragement of my research topic and his development of the proper statistical approach for the lifetime analysis. I wish 
to thank Andy Beretvas for all his assistance and for always being as concerned with my personal welfare as with that of my research. Gordon Thomson, as spokesman for the experiment, carried the experiment through from its earliest conception to its final physical realization and analysis. I will remember Mike Longo's wisdom in the arcana of apparatus, including the fact that sometimes scotch tape is better than epoxy.

My gratitude also goes to the several physicists, technicians and engineers who worked on the construction and setup of the apparatus. Thanks go to Pat Ward for designing and building the gamma trigger electronics, and to Dan Feyma for the construction of the counters. Alexander Pal deserves a prize for having to share several months of early morning "farm" shifts with a certain exasperating and impossible thesis student. The efforts of Tom Diehl, Jeff Duryea, Pak Ming Ho and Tony Carracappa were greatly appreciated, both in terms of their work in assembling apparatus, and in pulling innumerable shifts to allow us to run the experiment for eight long months.

I would also like to express my gratitude to the post-docs who assisted us in the experiment. I thank Keith Krueger for organizing the lead glass calibration runs and analysis. I thank Scott Teige for setting up the data summary tapes and providing advice and assistance.

My deepest gratitude goes to my fellow thesis students, Peter Border and Nancy Grossman. Together, they cleaned and repaired all the MWPCs, even after one of the chambers was made into a shish-kebob by a piece of Unistrut. They manned the majority of the 8 p.m. to 4 a.m. "vampire" shifts, making those quiet hours the most productive of the experiment. I now cherish their tolerance and patience of my numerous unwarranted tirades at 4:30 a.m. . Peter's wisdom, perseverance and good humor helped assemble an experiment out of the jumble of cables and electronics we faced in the summer of 1983. His expertise in figuring out a groundhog's affinity for cables was amazing. Nancy's long efforts on the gamma trigger electronics made them a working system. Her hard work and insights rescued us from many wrong turns and 
disasters during the experiment. Her continued efforts since the experiment to analyze the data has made my analysis infinitely easier.

My final thanks go to my friends and relatives for seeing me through this long process. My greatest appreciation is of my parents, Ronald and Sally Thorne, for raising me and supporting me when I doubted myself the most, and of my wife Kathy, who saw me through the last stages of my research. 


\section{Dedication}

I wish to dedicate this dissertation to the memory of my late father, Ronald Richard Thorne, and to my mother, Sally Ann Thorne. 


\section{Table of Contents}

Abstract

Acknowledgements $\quad$ iii

Dedication $\quad$ vi

List of Tables $\quad$ xii

List of Figures $\quad$ xiv

1 Introduction 1

1.1 Non-Leptonic Hyperon Decays .................. 2

1.1.1 The $\Delta I=1 / 2$ Rule $\ldots \ldots \ldots \ldots \ldots \ldots$

1.2 Theories of Non-leptonic Decays . . . . . . . . . . . . 7

1.3 Experimental Prospects .......................... 12

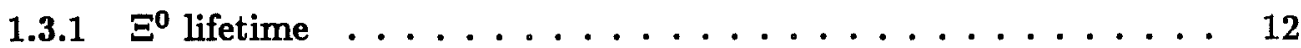

1.3.2 $\Xi^{0}$ Decay Asymmetry $\ldots \ldots \ldots \ldots$

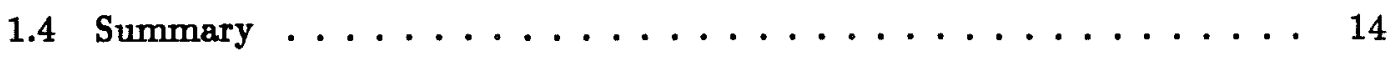

2 Apparatus $\quad 15$

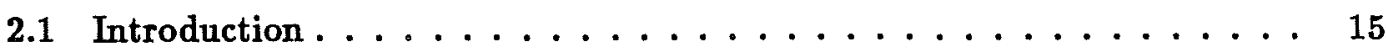

2.2 Proton Beamline ...................... 16 
2.3 Targets and Neutral Beam Channel . . . . . . . . . . . . . . 18

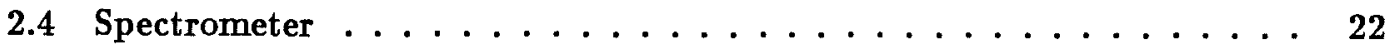

2.4 .1 Decay Volume ........................ 24

2.4.2 Charged-Particle Spectrometer . . . . . . . . . . 24

2.4.3 Photon Detector .................. 26

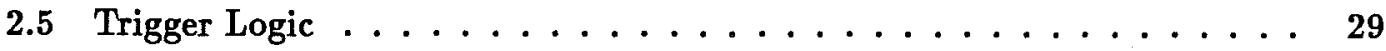

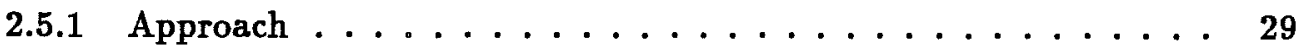

2.5.2 The F.C5 trigger ................... 31

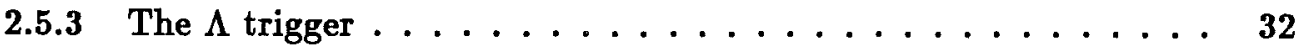

2.5.4 The $\Xi^{0}$ trigger $\ldots \ldots \ldots \ldots \ldots \ldots \ldots \ldots \ldots$

2.6 The $\gamma$ Trigger $\ldots \ldots \ldots \ldots \ldots \ldots \ldots \ldots \ldots \ldots \ldots$

2.6.1 Cluster-Counting Algorithm . . . . . . . . . . . . 34

$2.6 .2 \pi$-Killer Vetoes $\ldots \ldots \ldots \ldots \ldots \ldots \ldots \ldots$

2.6.3 Implementation . . . . . . . . . . . . . . 39

2.6.4 Performance .................... 39

2.7 Data Acquisition . . . . . . . . . . . . . . . . 40

2.7.1 MWPC Readout ................... 40

2.7.2 ADC and TDC Readout . . . . . . . . . . . . . 41

2.7.3 Latches and Scalers ................... 41

2.7.4 Recording the Event Data . . . . . . . . . . . . . 42

2.7.5 Between-Spill Readout . . . . . . . . . . . . . . 42

2.8 Experiment Operation ..................... 43

2.8.1 Chamber Alignment ... . . . . . . . . . . . 44

2.8.2 Lead Glass Calibration Runs . . . . . . . . . . . . . . . . 44

2.9 Detector Performance ..................... 45

2.9.1 Chamber Efficiency . . . . . . . . . . . . . 45 
2.9.2 Trigger Counter Efficiencies . . . . . . . . . . . . . 46

2.9.3 Lead Glass Energy and Position Resolution . . . . . . . . . . 46

3 Event Reconstruction

3.1 Introduction ...................... 52

$3.2 \Lambda$ reconstruction $\ldots \ldots \ldots \ldots \ldots \ldots \ldots \ldots \ldots \ldots$

3.2.1 Geometric Fit . ................... 53

3.2.2 Chamber centers ................... 55

3.2.3 Analysis Magnet Fields . . . . . . . . . . . . . 57

3.2.4 Decay Particle Identification $\ldots \ldots \ldots 6$

3.2.5 Kinematic $\Lambda$ fit $\ldots \ldots \ldots 63$

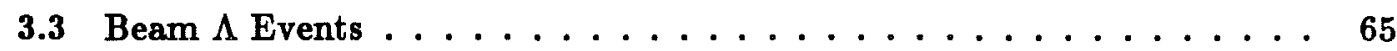

3.4 Photon Reconstruction . . . . . . . . . . . . . . 75

3.4.1 Cluster Selection . . . . . . . . . . . . . 75

3.4.2 Photon Cluster Identification . . . . . . . . . . 76

$3.5 \Xi^{0}$ Reconstruction $\ldots \ldots \ldots \ldots \ldots \ldots$

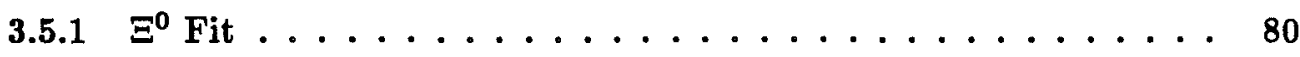

3.5.2 Bootstrap $\pi^{0}$ Calibration $\ldots \ldots \ldots . \ldots . \ldots 81$

3.5.3 $\Xi^{0}$ Events .......................... 82

4 Monte Carlo $\quad 100$

4.1 Neutral Beam Simulation . . . . . . . . . . . . . . . . 101

4.2 Particle Decay Simulation . . . . . . . . . . . . . . . 102

4.3 Charged Particle Simulation . . . . . . . . . . . . 105

4.3.1 Multiple Coulomb Scattering . . . . . . . . . . . . 105

4.3.2 Magnetic Fields . . . . . . . . . . . . . . . 106

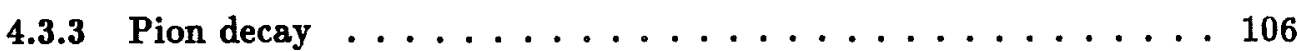


4.4 MWPC's and Scintillation Counters ................ 107

4.4.1 Chamber Inefficiencies . . . . . . . . . . . . . . 107

4.4.2 Multiple hits and chamber noise . . . . . . . . . . 108

4.4.3 Trigger counter efficiencies . . . . . . . . . . . . . 111

4.4.4 F.C5 and $\Lambda$ Trigger Simulation . . . . . . . . . . . . 114

4.5 Photons .............................. 114

$4.6 \Xi^{0}$ Trigger Simulation $\ldots \ldots \ldots \ldots \ldots \ldots \ldots \ldots \ldots$

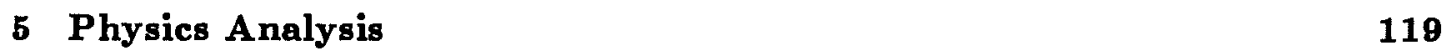

5.1 Lifetime Measurements - General . . . . . . . . . . . . . . . . 119

5.1.1 Lifetime Fitting Technique . . . . . . . . . . 120

$5.2 \Lambda$ Lifetime Fits ....................... 123

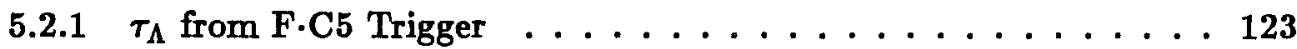

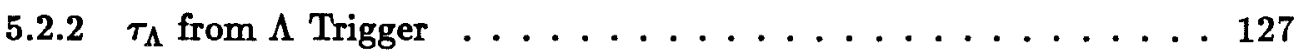

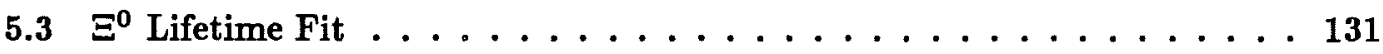

5.3.1 $\Xi^{0}$ Lifetime Using $\Xi^{0}$ Vertex $\ldots \ldots \ldots \ldots \ldots 131$

5.3.2 $\Xi^{0}$ Lifetime Using $\Lambda$ Vertex . . . . . . . . . . . 134

5.4 Asymmetry Measurements - General . . . . . . . . . . . 135

5.4.1 Hybrid $\alpha_{\Lambda} \alpha_{\Xi}$ Fit Technique . . . . . . . . . . . . 138

5.4.2 Initial $\alpha_{\Lambda} \alpha_{\Xi}$ Fit $\ldots \ldots \ldots . \ldots \ldots \ldots$

5.4.3 $\overrightarrow{\mathcal{P}}_{\Xi}$ and $\vec{B}_{\Lambda}$ Determination . . . . . . . . . . 141

5.4 .4 Final $\alpha_{\Lambda} \alpha_{\Xi}$ Fit . . . . . . . . . . . . . . . 148

$\begin{array}{llr}6 \text { Conclusions } & 151\end{array}$

$6.1 \Lambda$ Lifetime . . . . . . . . . . . . . . . . . . 151

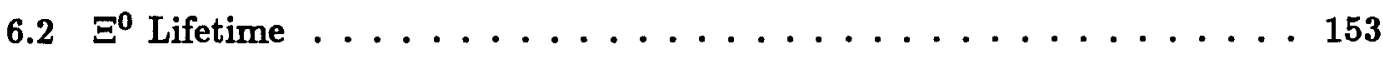

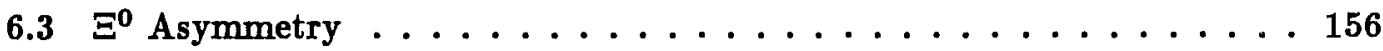


6.4 Theoretical Implications . . . . . . . . . . . . . . . . . 157

Appendix A. $\gamma$ Trigger Electronics

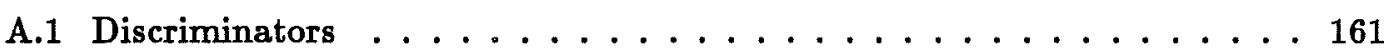

A.2 Veto Boards . . . . . . . . . . . . . . . . . . . 161

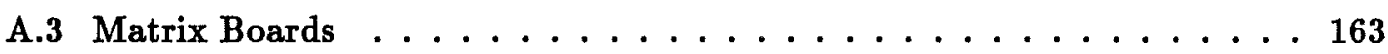

A.4 Summing Output Board ................... 166

$\begin{array}{ll}\text { References } & \mathbf{1 7 0}\end{array}$ 


\section{List of Tables}

1.1 Values for hyperon decay observables ............... 5

$1.2 \mathrm{~S}$ and $\mathrm{P}$-wave amplitudes $\ldots \ldots \ldots \ldots$

1.3 Experimental ratios of $\Delta I=3 / 2$ to $1 / 2$ amplitudes $\ldots \ldots \ldots \ldots$

1.4 S-wave amplitudes for PCAC-SU(3) and quark model fits compared to experimental measurements. ................ 9

1.5 P-wave amplitudes for PCAC, pole and diquark models compared to experimental results. ..................... 11

2.1 Target parameters $\ldots \ldots \ldots \ldots \ldots \ldots \ldots \ldots$

2.2 Z-positions of the spectrometer elements . . . . . . . . . . 23

2.3 Targets and magnet polarities during an 8 run cycle . . . . . . . . 43

2.4 Inefficiency (\%) of MWPC planes measured from reconstructed events . 46

3.1 Yields of reconstructed $\Lambda$ events $\ldots \ldots \ldots \ldots \ldots$

3.2 Yields of reconstructed $\Xi^{0}$ events $\ldots \ldots \ldots \ldots \ldots$

3.3 Analysis Magnet Field Parameters . . . . . . . . . . . . . . 59

3.4 Final beam $\Lambda$ criteria separated by target $\ldots \ldots \ldots \ldots \ldots$

3.5 Final $\Xi^{0}$ target pointing criteria separated by target $\ldots \ldots \ldots \ldots$

4.1 Average apparent 2-hit frequency (\%) in reconstructed events . . . . 108

4.2 Average probability(\%) of noise clusters per MWPC plane . . . . . . 109

5.1 Fits of $c \tau_{\Lambda}$ for DE and DW target F.C5 trigger data . . . . . . . 124 
5.2 Fits of $c \tau_{\Lambda}$ for combined F.C5 trigger data . . . . . . . . . 126

5.3 Systematic variation of $c \tau_{\Lambda}$ from F.C5 trigger data . . . . . . . 127

5.4 Fits of $c \tau_{\Lambda}$ for DE and DW target $\Lambda$ trigger events . . . . . . . . 128

5.5 Fits of $c \tau_{\Lambda}$ for combined $\Lambda$ trigger data . . . . . . . . . 128

5.6 Systematic variation of $c \tau_{\Lambda}$ from $\Lambda$ trigger data $\ldots \ldots \ldots$

5.7 Weighted average of $c \tau_{\Lambda}$ for F.C5 and $\Lambda$ trigger data . . . . . . . . 131

5.8 Fits of $c \Xi_{\Xi}$ from $\Xi^{0}$ vertex separated by target . . . . . . . . . . 132

5.9 Fits of $c \tau_{\Xi}$ from $\Xi^{0}$ vertex for combined DE+DW data . . . . . . . 132

5.10 Systematic variation of $c \tau_{\Xi}$ from $\Xi^{0}$ vertex fit $\ldots \ldots \ldots$. . . . . . 134

5.11 First pass on $\alpha_{\Lambda} \alpha_{\Xi}$ measurements, assuming $\overrightarrow{\mathcal{P}}_{\Xi}=0 \ldots \ldots 141$

5.12 Measurements of $\gamma_{\Xi} \mathcal{P}_{x}+B_{x}, \gamma_{\Xi} \mathcal{P}_{y}+B_{y}$ and $\gamma_{\Xi} \mathcal{P}_{z}+B_{z} \ldots \ldots 143$

$5.13 \gamma_{\Xi} \mathcal{P}_{z}$ and $B_{z}$ for DE and DW target event samples . . . . . . . 148

5.14 Final $\alpha_{\Lambda} \alpha_{\Xi}$ measurements . . . . . . . . . . . . . . . . . 149

6.1 Summary of experimental results and world averages . . . . . . 158 


\section{List of Figures}

1.1 Pseudo-scalar Spin $1 / 2$ Baryons . . . . . . . . . . . . . 2

1.2 Schematic for PCAC expansion of matrix elements . . . . . . . . 8

1.3 Quark annihilation, decay and penguin Feynman diagrams of non-leptonic weak decays . . . . . . . . . . . . . . . . . 10

2.1 E621 proton beamline optics $\ldots \ldots \ldots \ldots \ldots \ldots$

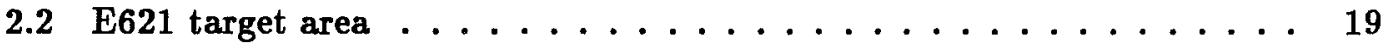

2.3 E621 neutral beam collimator . . . . . . . . . . . . . . . 21

2.4 The E621 spectrometer $\ldots \ldots \ldots \ldots \ldots \ldots \ldots$

2.5 Lead glass array and veto counters $\ldots \ldots \ldots \ldots \ldots$

2.6 Overlapped $\pi$-killer counters relative to the lead glass array. . . . . . . 28

2.7 E621 trigger diagram ..................... 30

2.8 Example cluster of lead glass blocks with signals due to an electromagnetic shower. ...................... 34

2.9 Example of a cluster edge-finding algorithm. . . . . . . . . . . 35

2.10 Application of edge-finding algorithm on three example clusters . . . . 36

2.11 Edge-finding algorithms used in the $\gamma$ trigger $\ldots \ldots \ldots \ldots$

2.12 Blocks in $\gamma$ trigger vetoed by hits in the $\pi$-killer array. . . . . . . . 38

2.13 Energy resolution of the lead glass array . . . . . . . . . . . 47

2.14 Linearity of lead glass array response . . . . . . . . . . . . . . 48 
2.15 Average position of the $e^{+}$and $e^{-}$tracks as a function of the calculated cluster center ....................... 50

3.1 Neutral vee fit topology .................... 53

$3.2 \chi^{2}$ per d.o.f. of the geometric fit for $\Lambda$ events $\ldots \ldots \ldots \ldots$

3.3 Effective bend plane $Z_{B E N D} \ldots \ldots \ldots \ldots \ldots$

3.4 Map of $\mathrm{Y}$ and Z-Axis Magnetic Field Components . . . . . . . . . 61

3.5 Distribution of $\mathrm{Y}$ residuals at chamber 5 for $\pi^{-}$tracks $\ldots \ldots \ldots$. . . 62

3.6 Invariant mass of beam $\Lambda$ events $\ldots \ldots \ldots \ldots$. . . . . . . 64

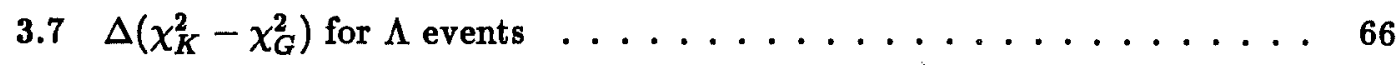

$3.8 R^{2}$ of beam $\Lambda$ 's from the downstream targets ........... 68

$3.9 \Delta \Theta_{x}$ and $\Delta \Theta_{y}$ distributions for DE target $\Lambda$ events $\ldots \ldots \ldots \ldots$

3.10 Momentum of DS beam $\Lambda$ events - F.C5 trigger . . . . . . . . . 71

3.11 Decay vertex $Z$ of DS beam $\Lambda$ events - F.C5 trigger . . . . . . . 72

3.12 Momentum of DS beam $\Lambda$ events $-\Lambda$ trigger . . . . . . . . 73

3.13 Decay vertex $Z$ of DS beam $\Lambda$ events $-\Lambda$ trigger $\ldots \ldots \ldots \ldots$

3.14 Number of blocks in photon showers for $\Xi^{0}$ events $\ldots \ldots \ldots$

3.15 Number of photon clusters in beam $\Lambda$ events $\ldots \ldots \ldots$

$3.16 \chi^{2}$ distribution for $\Xi^{0}$ fit $\ldots \ldots \ldots \ldots \ldots$

3.17 Distribution of reconstructed $\Xi^{0}$ decay vertices $\ldots \ldots \ldots$

3.18 Distribution of daughter $\Lambda$ decay vertices for $\Xi^{0}$ events . . . . . . 85

3.19 Momentum spectrum of daughter $\Lambda$ 's for $\Xi^{0}$ events $\ldots \ldots \ldots$

$3.20 \pi^{0}$ mass for accepted $\Xi^{0}$ events $\ldots \ldots \ldots \ldots \ldots$

3.21 Reconstructed $\pi^{0}$ mass for non-hole and hole $\Xi^{0}$ event samples $\ldots . . .88$

$3.22 \Xi^{0}$ mass distribution for accepted $\Xi^{0}$ events $\ldots \ldots \ldots \ldots$

$3.23 \Xi^{0}$ momentum distribution $\ldots \ldots \ldots \ldots \ldots$

3.24 Measured energy of photons in $\Xi^{0}$ events . . . . . . . . . . . 92 
$3.25 R_{\Xi}^{2}$ for $\Xi^{0}$ events from downstream targets . . . . . . . . . . 93

$3.26 \Delta X_{G L}$ and $\Delta Y_{G L}$ distributions for DE target $\Xi^{0}$ events $\ldots \ldots \ldots$

$3.27 R_{\Lambda}^{2}$ distribution for DS $\Xi^{0}$ events $\ldots \ldots \ldots \ldots$

3.28 Number of blocks in proton cluster in $\Xi^{0}$ events . . . . . . . . . . 97

3.29 Distribution of $\Delta(\gamma-p)$ for $\Xi^{0}$ events $\ldots \ldots \ldots \ldots$

4.1 Distribution of $\Xi^{0}$ events as a function of $\cos \theta \ldots \ldots \ldots$. . . . . . 104

4.2 Separation (cm) in X and $\mathrm{Y}$ of $p$ and $\pi^{-}$tracks at chamber C1 for $\Lambda$

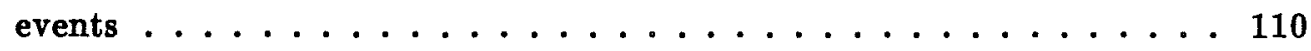

4.3 Distribution in $\mathrm{X}$ of $\pi^{-}$'s from $\Lambda$ trigger events at the B hodoscope . 112

4.4 Distribution in $\mathrm{X}$ of protons from $\Lambda$ trigger events at proton counters . . 113

4.5 Distribution in $\mathrm{X}$ of $\pi^{-}$tracks from $\Xi^{0}$ events at the $\mathrm{B}$ hodoscope $\ldots 117$

4.6 Rate of $V_{O R}$ vetoes vs. $x$ of $\pi^{-}$at the $B$ hodoscope . . . . . . . 118

5.1 Momentum dependence of $c \tau_{\Lambda}$ for F.C5 trigger events . . . . . . 125

5.2 Momentum dependence of $c \tau_{\Lambda}$ for $\Lambda$ trigger events . . . . . . . . 129

5.3 Momentum dependence of $c \tau_{\Xi}$ for $\Xi^{0}$ data . . . . . . . . . . 133

5.4 Definition of directions used in $\alpha_{\Xi}$ determination . . . . . . . . 137

$5.5 \alpha_{\Lambda} \alpha_{\Xi}$ as a function of $\Xi^{0}$ momentum, assuming $\overrightarrow{\mathcal{P}}_{\Xi}=0 \ldots \ldots 2$

$5.6 \gamma_{\Xi} \mathcal{P}_{x}+B_{x}$ as a function of $\Xi^{0}$ momentum $\ldots \ldots \ldots \ldots$

$5.7 \gamma_{\Xi} \mathcal{P}_{y}+B_{y}$ as a function of $\Xi^{0}$ momentum . . . . . . . . . 145

$5.8 \gamma_{\Xi} \mathcal{P}_{z}+B_{z}$ as a function of $\Xi^{0}$ momentum . . . . . . . . 146

$5.9 \alpha_{\Lambda} \alpha_{\Xi}$ as a function of $\Xi^{0}$ momentum . . . . . . . . . . . 150

6.1 Comparision of $\tau_{\Lambda}$ measurements $\ldots \ldots \ldots \ldots 2 \ldots \ldots \ldots$

6.2 Measurements of $\tau_{\Lambda}$ as a function of $\Lambda$ momentum $\ldots \ldots \ldots$

6.3 Comparision of $\tau_{\Xi^{0}}$ measurements . . . . . . . . . . . . 154

6.4 Measurements of $\tau_{\Xi^{0}}$ as a function of $\Xi^{0}$ momentum $\ldots \ldots \ldots 155$

6.5 Comparision of $\alpha_{\Lambda} \alpha_{\Xi}$ measurements ............... 156 
A.1 Block Diagram of the $\gamma$ Trigger Electronics . . . . . . . . . . . 160

A.2 Lead glass array blocks used in the $\gamma$ trigger . . . . . . . . . . . 162

A.3 Block diagram of $\gamma$ trigger Veto Board. . . . . . . . . . . 163

A.4 $\pi$-killer Overlap Circuit on the Veto Boards . . . . . . . . . . . . . . 164

A.5 Block Veto circuit on the Veto Boards . . . . . . . . . . . . . 165

A.6 Unit cell for a block on the Matrix Board . . . . . . . . . . . . . 167

A.7 Schematic of Summing Output Board . . . . . . . . . 168 


\section{Chapter 1}

\section{Introduction}

The field of particle physics has been engaged in studying the most basic constituents of matter and their interactions in order to understand the basic forces of nature. Over the past 30 years, this research has resulted in a remarkably successful set of theories. This "Standard Model" includes the Weinberg-Salam electro-weak gauge theory, which unified the description of the weak and electromagnetic forces; the quark model, which explained the hierarchy of hadronic particles; and Quantum Chromo-Dynamics (QCD) theory, which describes the strong force which bind the quarks together.

These theories have each been very successful at describing phenomena in particular regimes. The electro-weak theory gives excellent predictions of the interactions between leptons, which are particles that are not subject to the strong force. QCD only makes valid perturbative predictions of the dominant strong force for particle interactions involving large momentum transfers. It still remains to combine the electro-weak and QCD theories into a coherent structure which gives adequate predictions for all observed phenomena.

The weak decays of hadronic particles involve all these forces. The decays themselves are mediated by the weak interaction. During the decay, the quarks are undergoing strong interactions which bind them together into the new particles resulting from the 


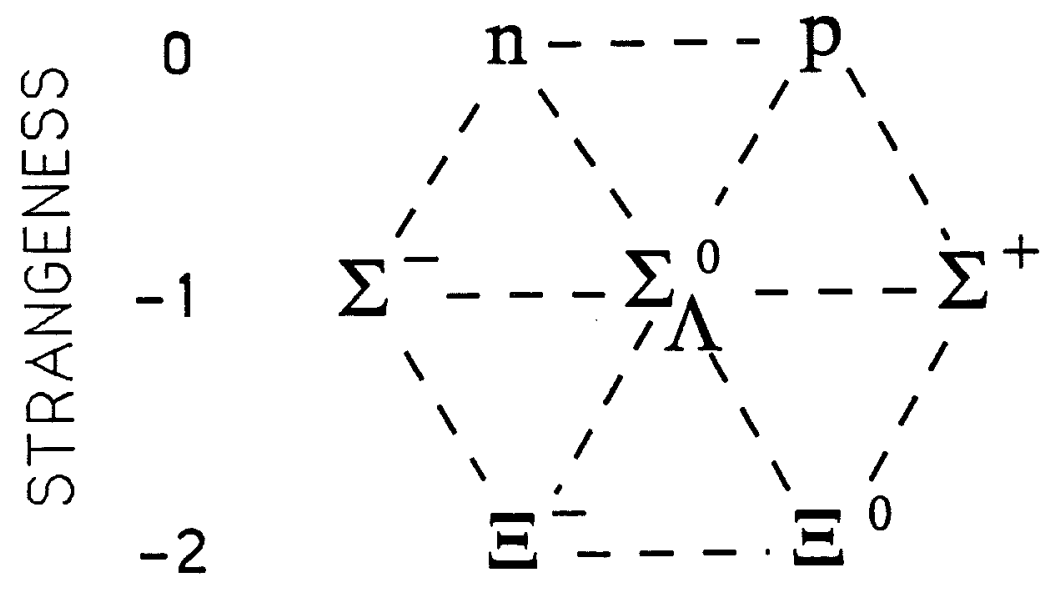

Figure 1.1: Pseudo-scalar Spin 1/2 Baryons

decay. Progress has been made on the theoretical understanding of these events which goes beyond simple phenomenological models. The prospect of improved theoretical predictions is cause to make more precise measurements of these decays in order to help refine the theories.

\subsection{Non-Leptonic Hyperon Decays}

Since their discovery over 30 years ago, the non-leptonic weak decays of the strange baryons have been used to study the physics of the weak interaction in particles (the hadrons) which also experience the strong force. These strange baryons, or "hyperons", include the ground-state members of the $\Lambda, \Sigma$ and $\Xi$ families of sub-atomic particles. They are similar to the more common protons(p) and neutrons(n), and occupy the same octet of spin 1/2 pseudo-scalar particles (Figure 1.1). The difference is that hyperons contain one or two "strange" quarks rather than only the lighter "up" and "down" quarks which make up the nucleons. The non-leptonic decays of the hyperons involve 
the decay of a strange quark via the weak interaction into up and down quarks, resulting in the decay of the "parent" hyperon $(B)$ into a "daughter" baryon $\left(B^{\prime}\right)$ and a $\pi$ meson, or pion.

These decay transitions have been characterized as due to a localized weak Hamiltonian $\left(H_{W}\right)$ operator. Because these baryons are spin $1 / 2$ and pions are spin zero, conservation of angular momentum $(\ell)$ restricts the weak Hamiltonian to having only $\ell=0$ ("S-wave") and $\ell=1$ ("P-wave") components. The weak Hamiltonian matrix element for these decays is of the form[1]

$$
\begin{aligned}
\mathcal{M} & =\left\langle B^{\prime}\left(p^{\prime}\right) \pi(q)\left|H_{W}\right| B(P)\right\rangle \\
& =\mathbf{G} m_{\pi}^{2} \bar{u}\left(p^{\prime}\right)\left[A+B \gamma_{5}\right] u(P)
\end{aligned}
$$

where $P, p^{\prime}$,and $q$ are the momenta of the parent, daughter baryon and pion respectively. $A$ and $B$ are the amplitudes of the $S$-wave and $P$-wave components. As weak decays are maximally parity-violating, chiral symmetry requires that the S-wave component be parity-violating and the $P$-wave be parity-conserving. $G$ is the coupling constant characterizing the weak decay, and $m_{\pi}$ is the rest mass of the charged pion.

The spin properties of the non-leptonic decays are best defined in the rest frame of the decaying hyperon of mass $M$. The decay matrix element can be defined in this frame as $[1]$

$$
\mathcal{M}=2 \mathrm{G} m_{\pi}^{2} M \psi_{B^{\prime}}^{\dagger}[S+P \vec{\sigma} \cdot \hat{n}] \psi_{B}
$$

where the $\psi$ 's are two-component spinors and $\hat{n}$ the unit vector along the momentum of the daughter baryon. $S$ and $P$ are also $S$-wave and $P$-wave amplitudes, and are related to $A$ and $B$ by

$$
\begin{aligned}
& S=\left(\frac{E+m^{\prime}}{2 M}\right)^{1 / 2} A \\
& P=\left(\frac{E-m^{\prime}}{2 M}\right)^{1 / 2} B
\end{aligned}
$$

where $E$ is the energy of the daughter baryon in the rest frame, and $m^{\prime}$ its rest mass. 
The $\mathrm{S}$ and $\mathrm{P}$-wave amplitudes can be expressed in terms of quantities which can be measured experimentally. One of these is the decay rate $(\Gamma)$ which is proportional to the magnitudes of the amplitudes:

$$
\Gamma=\frac{\mathrm{G}^{2} m_{\pi}^{4}}{2 \pi}|q|\left(|S|^{2}+|P|^{2}\right)
$$

The magnitude of $\left(\mathrm{G}^{2} m_{\pi}^{4} / 2 \pi\right)$ is $7.795 \times 10^{-15}$. The decay rate is determined experimentally by measuring the branching ratio $(B . R$.$) and lifetime (\tau)$, since $\Gamma=B . R . / \tau$. The relatively low rates of weak decays result in hyperon lifetimes on the order of $10^{-10}$ seconds. Precise measurements of the lifetimes have been possible because the highlyrelativistic hyperons produced from accelerators cover macroscopic distances of meters in the laboratory before decaying.

The mixing and relative phase of the $S$ and $P$ amplitudes was expressed by Lee and Yang[2] using three "decay asymmetry" parameters, $\alpha, \beta$, and $\gamma$, defined as:

$$
\alpha=\frac{2 \operatorname{Re}\left(S^{*} P\right)}{|S|^{2}+|P|^{2}} \quad \beta=\frac{2 \operatorname{Im}\left(S^{*} P\right)}{|S|^{2}+|P|^{2}} \quad \gamma=\frac{|S|^{2}-|P|^{2}}{|S|^{2}+|P|^{2}}
$$

The parameters satisfy the identity $\alpha^{2}+\beta^{2}+\gamma^{2}=1$. The values of these parameters are reflected in the spin asymmetry of the particles from these decays. In particular, the angular distribution of the daughter baryon, in the rest frame of the decaying baryon, is

$$
\frac{d n\left(B^{\prime}\right)}{d \Omega}=\frac{n\left(B^{\prime}\right)}{4 \pi}\left(1+\alpha \overrightarrow{\mathcal{P}}_{B} \cdot \hat{n}\right)
$$

where $\overrightarrow{\mathcal{P}}_{B}$ is the polarization of the parent and $\hat{n}$ is the unit vector along the daughter baryon momentum.

If the spins for an ensemble of identical spin $1 / 2$ particles are projected onto a given axis, the "polarization" of the spins of the ensemble relative to that axis can be determined. Polarization $(\mathcal{P})$ is defined as

$$
\mathcal{P}=\frac{N(\uparrow)-N(\downarrow)}{N(\uparrow)+N(\downarrow)}
$$




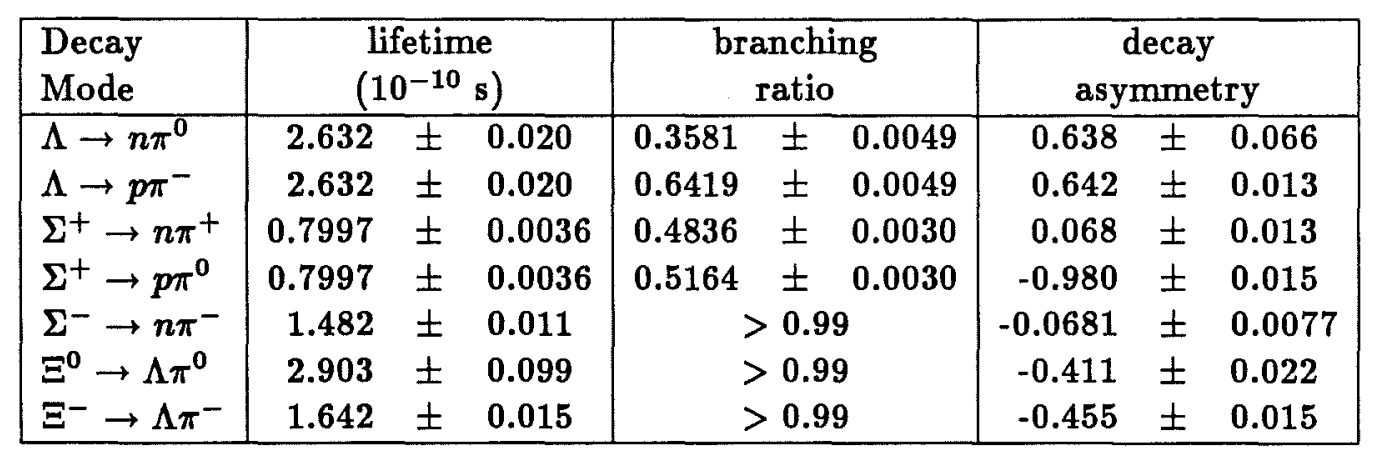

Table 1.1: Values for hyperon decay observables

where $N(\uparrow)$, and $N(\downarrow)$, are the number of particles with their projected spins parallel, and anti-parallel, to the axis. A polarization of zero corresponds to an isotropic distribution of spins. A polarization vector $(\overrightarrow{\mathcal{P}})$ can be formed from the components found with respect to three orthogonal axes $(x, y$ and $z)$.

The relationships in Equations 1.6 and 1.7 mean that the decay amplitudes $S$ and $P$, whose values should be predicted by theories of weak non-leptonic decays, can be determined experimentally through measurements of the branching ratio, lifetime, and decay asymmetry. Over many years, researchers have conducted experiments of increasing sophistication, resulting in quite precise measurements of the observables. The current world averages[3] are listed in Table 1.1 for the seven non-leptonic spin 1/2 hyperon decays. Any $\Sigma^{0}$ weak decays are overwhelmed by the dominant electromagnetic decay mode of $\Sigma^{0} \rightarrow \Lambda \gamma$. The largest remaining uncertainties are in the decay asymmetry of the $\Lambda \rightarrow n \pi^{0}$ mode, and in the lifetime of the $\Xi^{0}$.

These measurements have been used to derive the $\mathrm{S}$ and $\mathrm{P}$-wave amplitudes listed in Table 1.2. Time-reversal invariance has been assumed, thus $A$ and $B$ are relatively real and $\beta=0$.

\subsubsection{The $\Delta I=1 / 2$ Rule}

Despite having different electric charges, the proton and neutron are nearly identical with respect to the strong force which binds them together in the nucleus. This is now 


\begin{tabular}{|l|rcc|rcc|}
\hline Decay & \multicolumn{2}{|c|}{ A (S-wave) } & \multicolumn{3}{|c|}{ B (P-wave) } \\
\hline$\Lambda \rightarrow n \pi^{0}$ & -1.07 & \pm & 0.01 & -7.14 & \pm & 0.56 \\
$\Lambda \rightarrow p \pi^{-}$ & 1.47 & \pm & 0.01 & 9.98 & \pm & 0.24 \\
$\Sigma^{+} \rightarrow n \pi^{+}$ & 0.06 & \pm & 0.01 & 19.06 & \pm & 0.08 \\
$\Sigma^{+} \rightarrow p \pi^{0}$ & 1.47 & \pm & 0.05 & -12.07 & \pm & 0.56 \\
$\Sigma^{-} \rightarrow n \pi^{-}$ & 1.93 & \pm & 0.01 & -0.65 & \pm & 0.08 \\
$\Xi^{0} \rightarrow \Lambda \pi^{0}$ & 1.55 & \pm & 0.03 & -5.56 & \pm & 0.32 \\
$\Xi^{-} \rightarrow \Lambda \pi^{-}$ & 2.03 & \pm & 0.01 & -7.87 & \pm & 0.28 \\
\hline
\end{tabular}

Table 1.2: $\mathrm{S}$ and $\mathrm{P}$-wave amplitudes

attributed to the symmetry of the strong interaction under the exchange of $u$ and $d$ quarks. This invariance has been characterized by the quantum number Isospin $(I)$, which together with its "third-component" $I_{3}$ have the same algebraic and symmetry properties as a "true" spin. The two nucleons form an $I=1 / 2$ isospin doublet, the proton having $I_{3}=+1 / 2$, the neutron $I_{3}=-1 / 2$. This formalism has been extended to all the light hadrons. Hadrons with the same total number of $u$ and $d$ quarks form isospin multiplets. The $\Lambda$ is an $I=0$ singlet, the $\Xi^{\prime} s$ are $I=1 / 2$, while the $\Sigma^{\prime} s$ and $\pi^{\prime} s$ are $I=1$.

In non-leptonic weak decays, the isospin of the state is changed, the amount of change being either $1 / 2$ or $3 / 2$, and the weak Hamiltonian can be separated into $\Delta I=1 / 2$ and $\Delta I=3 / 2$ components. Using Clebsch-Gordon coefficients, the $S$ and $P$-wave decay amplitudes can be expressed in terms of these isospin amplitudes[4]. The $\Lambda$ and $\Xi$ decays have similar decompositions for the S-wave amplitudes:

$$
\begin{aligned}
A\left(\Lambda_{0}^{0}\right) & =\frac{1}{\sqrt{2}} A_{\Lambda}^{(1)}-\frac{1}{\sqrt{2}} A_{\Lambda}^{(3)} & A\left(\Xi_{0}^{0}\right) & =\frac{1}{\sqrt{2}} A_{\Xi}^{(1)}-\frac{1}{\sqrt{2}} A_{\Xi}^{(3)} \\
A\left(\Lambda_{-}^{0}\right) & =A_{\Lambda}^{(1)}+\frac{1}{2} A_{\Lambda}^{(3)} & A\left(\Xi_{-}^{-}\right) & =A_{\Xi}^{(1)}+\frac{1}{2} A_{\Xi}^{(3)}
\end{aligned}
$$

The same relations hold for the P-wave amplitudes. The notation on $\Lambda$ and $\Xi$ uses the charge of the parent (superscript) and decay pion (subscript) to identify the decay mode. The (1),(3) superscripts refer to the $\Delta I=1 / 2,3 / 2$ parts respectively. The weak interaction itself has no inherent isospin preference, so a priori one expects the $\Delta I=1 / 2$ 


\begin{tabular}{|c|r|r|}
\hline Hyperon & \multicolumn{1}{|c|}{$\mathrm{A}^{(3)} / \mathrm{A}^{(1)}$} & \multicolumn{1}{c|}{$\mathrm{B}^{(3)} / \mathrm{B}^{(1)}$} \\
\hline$\Lambda$ & $0.027 \pm 0.008$ & $0.030 \pm \mathbf{0 . 0 3 7}$ \\
$\Sigma$ & $-0.061 \pm 0.024$ & $-0.074 \pm \mathbf{0 . 0 2 7}$ \\
$\Xi$ & $-0.048 \pm 0.014$ & $\mathbf{0 . 0 3 3} \pm \mathbf{0 . 0 2 5}$ \\
\hline
\end{tabular}

Table 1.3: Experimental ratios of $\Delta I=3 / 2$ to $1 / 2$ amplitudes for hyperon non-leptonic decays. Derived from the $\mathrm{S}$ and $\mathrm{P}$-wave amplitudes of Table 1.2.

and $\Delta I=3 / 2$ amplitudes to be equal. However, it was found that the experimental lifetimes and asymmetries were consistent (Table 1.3) with $\Delta I=3 / 2$ amplitudes which are only a few percent of the the $\Delta I=1 / 2$ amplitudes. This empirical isospin selection rule, termed the " $\Delta I=1 / 2$ rule", also holds for the non-leptonic kaon decays $(K \rightarrow$ $\pi \pi$ ). Within the standard model, this $\Delta I=1 / 2$ dominance implies that there are large corrections to the weak Hamiltonian due to the strong interaction.

\subsection{Theories of Non-leptonic Decays}

The non-leptonic hyperon decays have proven to be a frustrating problem for the theorists. A number of theoretical models have been proposed, each addressing a particular aspect of the problem. None has been successful at explaining both the $S$ and $P$ wave amplitudes in a comprehensive manner.

The basic phenomenological approach in calculating the $S$ and $P$-wave amplitudes uses the Partially Conserved Axial Current (PCAC) model. This model invokes the general properties of the chiral symmetries of the weak decays to solve for the amplitudes, without requiring detailed knowledge of the interactions. Using the PCAC structure helps to reveal which processes are important for each of the calculations. The following presentation is adapted from a recent review by Donoghue[5]. The PCAC analysis makes the assumption that the weak Hamiltonian matrix elements can be expanded in the limit that the decay pion's momentum is zero. This "soft-pion" theorem is justified 


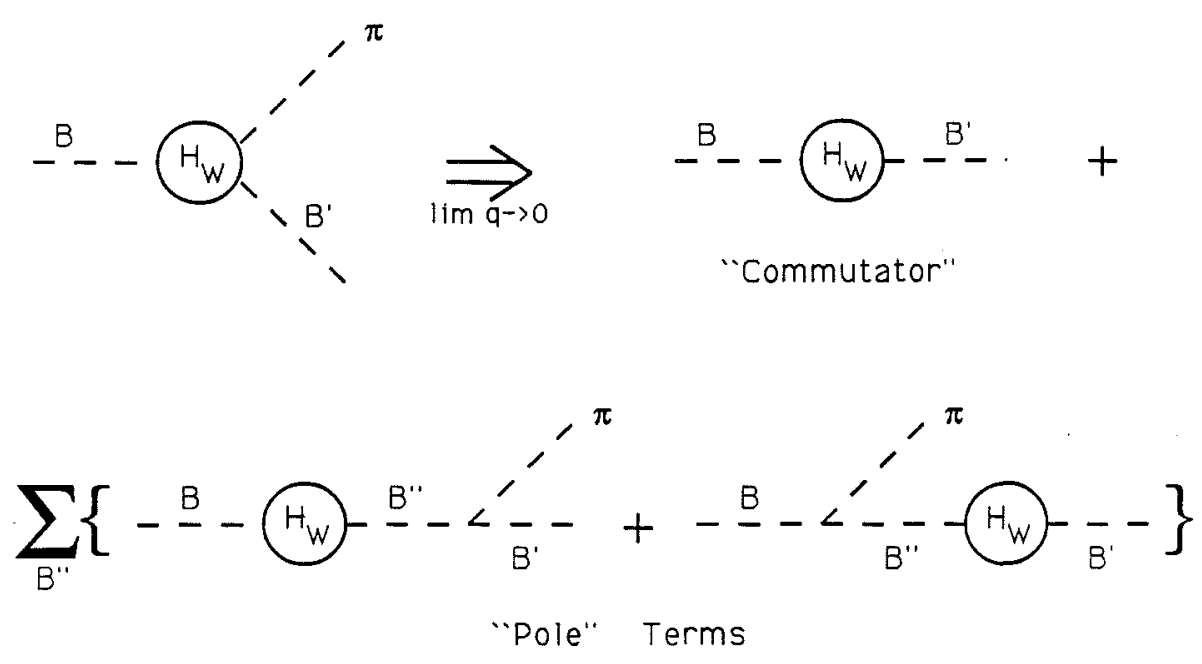

Figure 1.2: Schematic for PCAC expansion of matrix elements

by the fact that the pions in these decays have a typical momentum of about one hundred $\mathrm{MeV} / \mathrm{c}$ in the center of mass. Through this technique, the individual $B \rightarrow B^{\prime} \pi$ matrix elements can be expanded in terms of weak Hamiltonian matrix elements which are only between baryons. This expansion is shown schematically in Figure 1.2, where the first term on the right-hand side is the "commutator" between the parent $(B)$ and decay $\left(B^{\prime}\right)$ baryons, while the other two are the "pole" terms, which are summed over intermediate baryon $\left(B^{\prime \prime}\right)$ states. Equation 1.11 is the expansion for the parity-violating (P.V.) S-wave amplitude and Equation 1.12 is for the parity-conserving (P.C.) P-wave amplitude.

$$
\begin{aligned}
& \lim _{q \rightarrow 0}\left\langle B^{\prime} \pi\left|H_{W}^{P .} V_{\cdot}\right| B\right\rangle=\frac{-i}{2 F_{\pi}}\left\langle B^{\prime}\left|H_{W}^{P . C} \cdot\right| B\right\rangle \\
& +\sum_{B^{\prime \prime}}\left[\frac{\left\langle B^{\prime} \pi \mid B^{\prime \prime}\right\rangle\left\langle B^{\prime \prime}\left|H_{W}^{P} V_{\cdot}\right| B\right\rangle}{m_{B^{\prime \prime}}-m_{B}}+\frac{\left\langle B^{\prime}\left|H_{W}^{P} V_{\cdot}\right| B^{\prime \prime}\right\rangle\left\langle B^{\prime \prime} \pi \mid B\right\rangle}{m_{B^{\prime \prime}}-m_{B}}\right] \\
& \lim _{q \rightarrow 0}\left\langle B^{\prime} \pi\left|H_{W}^{P_{W} C \cdot}\right| B\right\rangle=\frac{-i}{2 F_{\pi}}\left\langle B^{\prime}\left|H_{W}^{P_{W} V} \cdot\right| B\right\rangle \\
& +\sum_{B^{\prime \prime}}\left[\frac{\left\langle B^{\prime} \pi \mid B^{\prime \prime}\right\rangle\left\langle B^{\prime \prime}\left|H_{W}^{P . C} \cdot\right| B\right\rangle}{m_{B^{\prime \prime}}-m_{B}}+\frac{\left\langle B^{\prime}\left|H_{W}^{P . C} \cdot\right| B^{\prime \prime}\right\rangle\left\langle B^{\prime \prime} \pi \mid B\right\rangle}{m_{B^{\prime \prime}}-m_{B}}\right]
\end{aligned}
$$

Note that the commutator terms have the opposite parity than that of the original 


\begin{tabular}{|l|c|c|r|}
\hline Decay & SU(3)-PCAC & Quark Model & Measured (A) \\
\hline$\Lambda \rightarrow n \pi^{0}$ & -1.08 & - & $-1.07 \pm 0.01$ \\
$\Lambda \rightarrow p \pi^{-}$ & 1.53 & 1.40 & $1.47 \pm 0.01$ \\
$\Sigma^{+} \rightarrow n \pi^{+}$ & 0 & 0 & $0.06 \pm 0.01$ \\
$\Sigma^{+} \rightarrow p \pi^{0}$ & 1.43 & 1.49 & $1.47 \pm 0.05$ \\
$\Sigma^{-} \rightarrow n \pi^{-}$ & 2.03 & 2.00 & $1.93 \pm 0.01$ \\
$\Xi^{0} \rightarrow \Lambda \pi^{0}$ & 1.42 & - & $1.55 \pm 0.03$ \\
$\Xi^{-} \rightarrow \Lambda \pi^{-}$ & 2.00 & 2.19 & $2.03 \pm 0.01$ \\
\hline
\end{tabular}

Table 1.4: S-wave amplitudes for PCAC-SU(3)[7] and quark model fits compared to experimental measurements of $A$ from Table 1.2 .

matrix element. This is due to the left-handed helicity of the weak interaction.

If one assumes that $\mathrm{SU}(3)$ flavor symmetry is valid (i.e. that $\mathrm{u}, \mathrm{d}$ and $\mathrm{s}$ quarks all have the same mass), then it has been shown[6] that the parity-violating baryon-baryon matrix elements $\left\langle B^{\prime}\left|H_{W}^{P . V} \cdot\right| B\right\rangle$ vanish. Applying this Lee-Swift Theorem to Equation 1.11 means that the S-wave amplitudes are dominated by the commutator term, while applying it to Equation 1.12 results in the P-wave amplitudes being dominated by the pole terms.

The S-wave terms have been calculated in several ways. The simplest model makes a two-variable parameterization of the baryon-baryon matrix elements, and fits them to the data. This SU(3)-PCAC fit[7] agrees quite well with the experimental data (Table 1.4). With the development of the quark model of the hadrons and of QCD, attempts have been made to calculate the baryon-baryon matrix elements using quark wavefunctions. It was found $[8]$ that there were significant corrections due to pole terms from $1 / 2^{-}$baryons. These pole terms vanish in the soft-pion limit. As shown in Table 1.4, these models also give reasonable results. Still unsettled is the fact that experimentally the S-wave baryon-baryon amplitudes have a form factor[7] ratio of $f / d=-2.4$, while the quark models have an inherent $f / d=-1$.

The quark model provided an understanding of how the dominance of $\Delta I=1 / 2$ amplitudes could arise from the dynamical interactions of quarks. The basic quark 

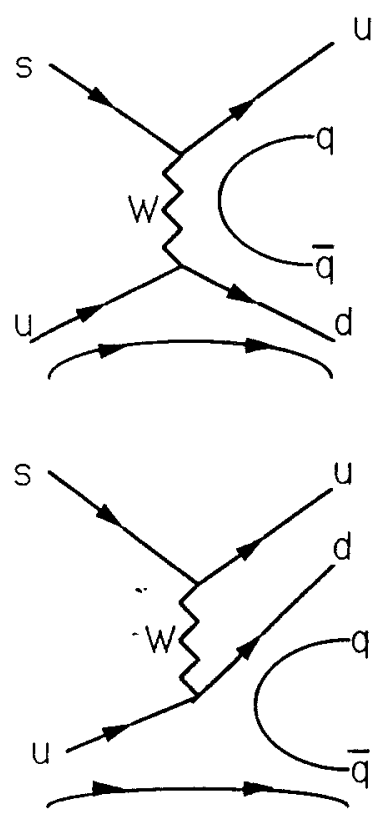

ANNIHILATION
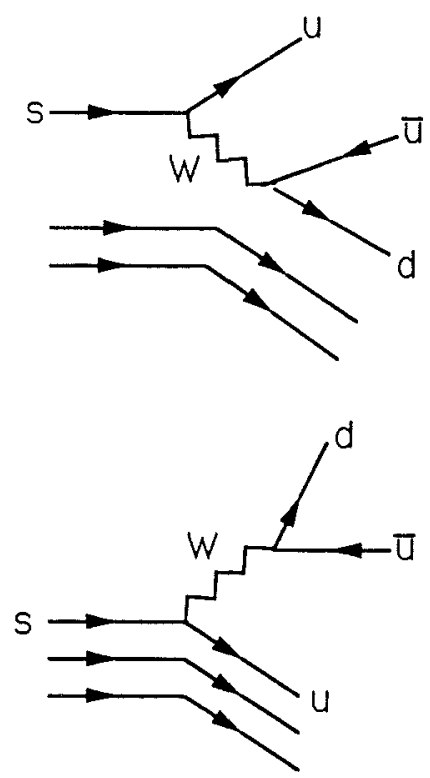

QUARK DECAY
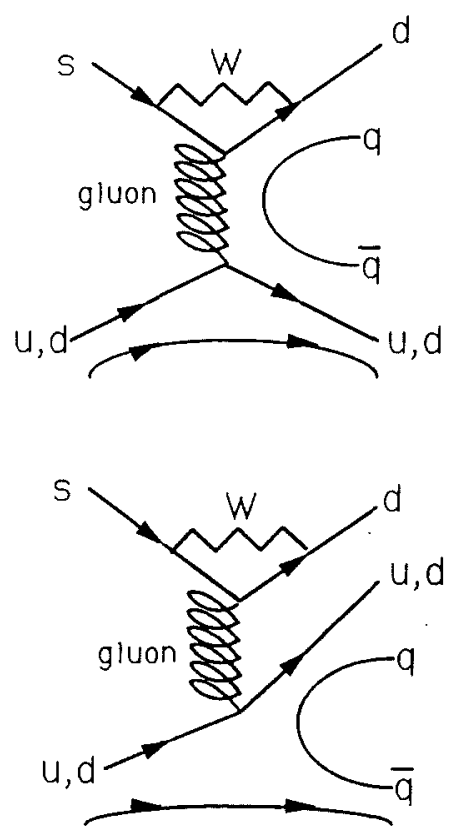

PENQUIN

Figure 1.3: Quark annihilation, decay and penguin Feynman diagrams of non-leptonic weak decays

interaction diagrams in non-leptonic baryon decay are quark annihilation, decay and the "penguin" diagrams involving gluon exchange (Figure 1.3). The "W" in the diagrams represents the charged vector boson which carries the weak force, just as the photon is the carrier of the electro-magnetic force. The penguin diagrams are inherently only $\Delta I=1 / 2$, while the other two diagrams embody both $\Delta I=1 / 2$ and $\Delta I=3 / 2$ amplitudes. In fact, if only the weak interaction is considered, these diagrams should have equal amount of $\Delta I=1 / 2$ and $\Delta I=3 / 2$. But $Q C D$ theory has an added symmetry which is termed "color". Because the baryons must be antisymmetric under the exchange of color, it has been shown[9] that the $\Delta I=3 / 2$ components are suppressed.

The $\mathrm{P}$-wave amplitudes have been much more difficult to calculate successfully. 


\begin{tabular}{|l|c|c|c|c|c|}
\hline Decay & PCAC & \multicolumn{2}{|c|}{ Pole Models } & Diquarks & Measured \\
Mode & Finjord(80) & Donoghue(86) & Nardulli(88) & Dosch(88) & $(B)$ \\
\hline$\Lambda \rightarrow n \pi^{0}$ & $-8.12 \pm 0.40$ & -5.2 & -5.78 & - & $-7.14 \pm 0.56$ \\
$\Lambda \rightarrow p \pi^{-}$ & $\mathbf{1 0 . 6 0} \pm \mathbf{0 . 5 6}$ & 7.4 & $\mathbf{8 . 0 8}$ & - & $\mathbf{9 . 9 8} \pm 0.24$ \\
$\Sigma^{+} \rightarrow n \pi^{+}$ & $\mathbf{0 . 1 6} \pm \mathbf{0 . 3 3}$ & 12.8 & $\mathbf{2 0 . 7 4}$ & 18.7 & $\mathbf{1 9 . 0 6 \pm 0 . 0 8}$ \\
$\boldsymbol{\Sigma}^{+} \rightarrow p \pi^{0}$ & $-4.91 \pm 0.17$ & -9.2 & -14.79 & -11.9 & $-\mathbf{1 2 . 0 7} \pm 0.56$ \\
$\Sigma^{-} \rightarrow n \pi^{-}$ & $\mathbf{- 5 . 3 2} \pm 0.23$ & -0.4 & -0.14 & - & $-0.65 \pm 0.08$ \\
$\Xi^{0} \rightarrow \Lambda \pi^{0}$ & $-1.06 \pm 0.56$ & -7.7 & -4.25 & -6.5 & $-5.56 \pm 0.32$ \\
$\Xi^{-} \rightarrow \Lambda \pi^{-}$ & $-1.50 \pm 0.78$ & -11.3 & -6.31 & -9.1 & $-7.87 \pm 0.28$ \\
\hline
\end{tabular}

Table 1.5: $\mathrm{P}$-wave amplitudes for PCAC[10], pole[7,11] and diquark[12] models compared to experimental measurements of $B$ from Table 1.2.

The PCAC model, using the values for baryon-baryon elements from the S-wave fits, makes predictions[10] which are significantly different than the experimental results (Table 1.5). Various solutions have been explored. Some theorists ascribe the discrepancy to terms which have been left out of the expansions because they vanish as $q \rightarrow 0$. Donoghue[7] allows the $\mathrm{P}$-wave fits to the pole terms to vary the values of $f$ and $d$, which results in an improved fit (Table 1.5). These particular values for $f$ and $d$ do not give a simultaneously good fit to the S-wave amplitudes, however. Other authors, such as Nardulli[11], have ascribed the differences to long-range strong-interaction effects. They take these into account by increasing the number of excited baryons poles summed over to include several other particle multiplets. Their results also show improved agreement, but this may only be a consequence of the increased number of degrees of freedom, and such extended pole models do not contribute to an understanding of the processes involved. Dosch et al.[12] have suggested considering the baryons as made up of a bound quark pair, the "diquark", and a another quark, as a way to take the strong quark interactions into account. Their results, which are only reported for modes where other non-soft-pion effects are small, are encouraging.

The difficulty in using QCD theory to calculate quark dynamics is that perturbative QCD theory has a "cutoff" energy of about $1 \mathrm{GeV}$, while these decays occur on energy scales of $\sim 200 \mathrm{MeV}$. Below this cutoff, which is set by the strength of the coupling 
constant $\alpha_{S}, \mathrm{QCD}$ interactions must be calculated in a non-perturbative fashion. It is hoped that the current work on "lattice QCD", which is showing progress in calculating the amplitudes in the similar $K \rightarrow \pi \pi$ decays will eventually yield definitive explanations of both the S and P-wave amplitudes.

\subsection{Experimental Prospects}

The recent improvements in the theoretical understanding and predictions of the nonleptonic decay amplitudes, especially for the $P$-waves, are cause to examine the existing data to see where improved measurements might be in order.

The $\Xi$ multiplet of $\Xi^{0}$ and $\Xi^{-}$is perhaps the best place to concentrate on making improved measurements. Because both of these particles have a daughter baryon, the $\Lambda$, which also undergoes a weak decay, the decay asymmetries $\left(\alpha_{\Xi}\right)$ can be measured to high precision without a detailed knowledge of the polarization of the $\Xi$ (See Section 1.3.2). Because both the $\Xi^{0}$ and $\Xi^{-}$decays end up in the same $I=1$ isospin state, small corrections to the $S$ and $P$-wave decay amplitudes, which are due to $\Lambda-\pi$ final-state scattering, cancel out when the amplitudes for the two decays are compared.

\subsection{1 $\Xi^{0}$ lifetime}

An improved measurement of the lifetime of the $\Xi^{0}$ is needed. The current world average for $\tau_{\Xi^{0}}$ is $2.903 \pm 0.099 \times 10^{-10} \mathrm{~s}$, which has an uncertainty five times larger than that for any of the other octet hyperon weak decays (See Table 1.1). Several authors[13,14] had observed how this imprecision limits the accuracy of experimental determinations of the $\Delta I=3 / 2$ component in $\Xi$ S-wave decay amplitudes(Table 1.3). The last measurement of $\tau_{\Xi 0}$ was reported in $1977[15]$, based on a sample of 6300 events. Since that time, experiments have been conducted at Fermilab involving 100,000's of such decays[16]. Hence, it is now feasible to collect sufficient numbers of $\Xi^{0} \rightarrow \Lambda \pi^{0}$ events to significantly 
reduce the uncertainty on $\tau_{\Xi^{0}}$.

The measurement of hyperon lifetimes is typically done by having a high-energy beam of hadrons, usually protons, interact in a small target to produce an intense beam which includes hyperons. The hyperons decay in flight in the laboratory, resulting in charged particles and photons whose positions and energy are measured by detectors. These quantities are used to reconstruct the decay vertex and momentum of each parent baryon which decayed. The decay vertexes $(z)$ for particles of a given momentum $(p)$ have an exponential distribution, $\exp (-m z / p \tau)$, which is a function of the lifetime $\tau$.

The $\Xi^{0}$ lifetime measurement is complicated by the fact that the $\Xi^{0}$ is a neutral particle which decays into two other neutral particles, the $\Lambda$ and $\pi^{0}$. Thus the decay vertex cannot be determined directly from the tracks of charged particles left in the detectors. The $\Xi^{0}$ vertex can be extracted from a kinematic fit using its daughter $\Lambda$, reconstructed from the $p$ and $\pi^{-}$it decays into, and the energies and positions of the two photons in a calorimeter. The $\Xi^{0}$ lifetime can also be measured from the distribution of the daughter $\Lambda$ vertices as a function of the $\Xi^{0}$ and $\Lambda$ momentum.

\subsection{2 $\Xi^{0}$ Decay Asymmetry}

The measurement of $\alpha$ in hyperon decays, as shown in Equation 1.8, is dependent on knowing the polarization of the parent baryon. In the case of the $\Xi^{\prime} s$, however, the subsequent decay of the daughter baryon can be used to measure the asymmetry even without having a beam of polarized $\Xi^{\prime} s$. As shown before, the angular distribution of the daughter protons in $\Lambda \rightarrow p \pi^{-}$is

$$
\frac{d n(p)}{d \Omega}=\frac{n(p)}{4 \pi}\left(1+\alpha_{\Lambda} \overrightarrow{\mathcal{P}}_{\Lambda} \cdot \hat{p}\right)
$$

where $\hat{p}$ is a unit vector along the proton momentum in the $\Lambda$ rest frame. Lee and Yang[2] showed that the daughter baryon is polarized in hyperon decays. For unpolarized 
$\Xi^{0}$ 's, the polarization of the daughter $\Lambda$ is just:

$$
\overrightarrow{\mathcal{P}}_{\Lambda}=\alpha_{\Xi_{0} 0} \hat{\Lambda}
$$

where $\hat{\Lambda}$ is the unit vector along the $\Lambda$ momentum. When substituted into Equation 1.13 , this definition of $\overrightarrow{\mathcal{P}}_{\Lambda}$ gives:

$$
\frac{d n(p)}{d \Omega}=\frac{n(p)}{4 \pi}\left(1+\alpha_{\Lambda} \alpha_{\Xi^{0}} \hat{\Lambda} \cdot \hat{p}\right)
$$

Thus the product of the asymmetries, $\alpha_{\Lambda} \alpha_{\Xi^{0}}$, can be determined by measuring the proton distribution in the $\Lambda$ rest frame. This technique avoids relying the reconstruction of the $\pi^{0}$, which often has much poorer resolution than that of the charged particles.

The existing world average for $\alpha_{\Lambda} \alpha_{\Xi^{\circ}}$ is $-0.264 \pm 0.006$, which given the existing average $\alpha_{\Lambda}$ of $0.642 \pm 0.013$ implies a value for $\alpha_{\Xi^{0}}$ of $-0.413 \pm 0.022$. This average is dominated by the results of one experiment[13], so it would be useful to re-measure it.

\subsection{Summary}

This thesis will present the results of precision $(\sim 1-2 \%)$ measurements of the $\Xi^{0} \rightarrow \Lambda \pi^{0}$ lifetime and decay asymmetry done in an experiment at the Fermi National Accelerator Lab (Fermilab). It will also present a precision measurement of the $\Lambda$ lifetime. The impact of these new results on the existing $\Delta I=1 / 2$ rule for $\Xi$ decays will then be briefly discussed. 


\section{Chapter 2}

\section{Apparatus}

\subsection{Introduction}

A large, relatively unbiased sample of $\Xi^{0}$ decays was required to measure the $\Xi^{0}$ decay properties of lifetime and asymmetry. In this experiment, a beam of protons interacted in a target producing a high-intensity collimated beam of neutral particles, which included $\Xi^{0}$ 's as well as $\Lambda$ 's, kaons, neutrons and photons. The $\Xi^{0}$ particle typically (> 99\% of the time) decays into a $\Lambda$ and $\pi^{0}$ particles. The $\pi^{0}$ decays immediately ( $\left.<10^{-16} \mathrm{~s}\right)$ into two photons $\left(\gamma^{\prime}\right.$ s), while the $\Lambda$ decays weakly, two-thirds of the time into a proton and $\pi^{-}$. A magnetic spectrometer measured the momenta and locations of the $p$ and $\pi^{-}$charged particles to allow the reconstruction of the parent $\Lambda$. A segmented lead glass calorimeter measured the energy and position of the photons, from which the $\pi^{0}$ could be reconstructed. A decay "event" was signaled by the presence of at least two oppositely-charged particles in the spectrometer and at least two showers in the lead glass array. This signal triggered the digitization and acquisition of the detector information. A computer read out this data and wrote it onto magnetic tapes for later analysis.

These data were taken during Fermilab experiment E621 in 1985. The main physics 
goal of that experiment was to measure CP violation in $K_{\pi 3}$ decays $\left(K \rightarrow \pi^{+} \pi^{-} \pi^{0}\right)$. The apparatus was optimized towards that end, and any modification required to make this measurement had to be compatible with that design. I will discuss below only those aspects of the apparatus relevant to the $\Xi^{0}$ and $\Lambda$ events.

\subsection{Proton Beamline}

The Fermilab accelerator provided beams of $800 \mathrm{GeV} / \mathrm{c}$ protons. These were extracted during a 22 second spill, with a typical repetition period of 60 seconds. Our experiment was located in the Proton Center (PC) beamline, which received from $10^{10}$ to $3 \times 10^{11}$ protons/spill, depending on our running conditions.

The PC beamline split the incoming beam into two nearly parallel beams. The beam direction was roughly north-east, termed Site North. The two separate beams were termed the East and West beams, owing to their relative positions at the targets. These beams were focussed as they were transported to a pair of targets upstream of the collimator. A schematic layout of the elements in both plan and elevation views is provided in Figure 2.1.

The splitting of the beam was initiated by an electrostatic septum in Enclosure P01, which split the beam vertically. The beams then drifted downstream for 538 meters, separating enough to match the double aperture of a pair of Lambertson magnets and the beginning on the next enclosure. Lambertson magnets are a special design with two beam holes such that one hole has a magnetic field, while the other hole, of circular cross-section, has no field. In our case, the upper hole had no field, through which the East beam passed. The lower hole, with a field, bent the West beam to the left. The dipole (bending) magnets in enclosure P01 were used to direct the beam to the septum. The quadrupole (focussing) magnets were to provide the best match of the beams to the apertures of the Lambertson magnet. This was done to minimize the production of muons and other secondaries which could become unwanted backgrounds 


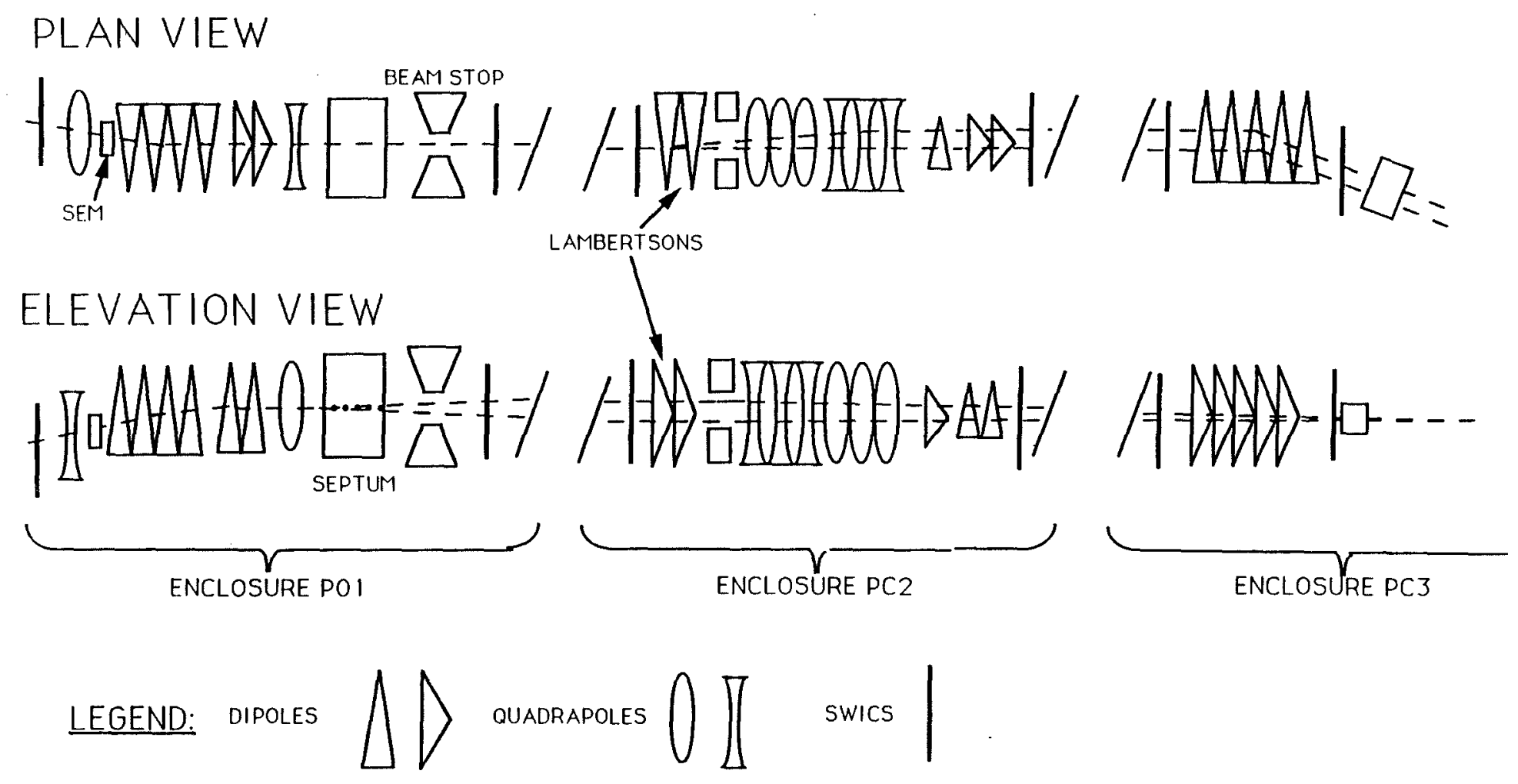

Figure 2.1: E621 proton beamline optics 
in our detector.

The magnets after the Lambertson were used to direct the beams onto the targets. The quadrupoles in $\mathrm{PC} 2$ rotated the beams into the horizontal plane, as well as focussed them transversely. A careful study of the beam tuning was made to ensure that both beams focussed at the targets located at the entrance to the neutral beam channel. The trim dipoles in PC2 were used to adjust the beam positions at the targets, while the string of dipoles in PC3 bent the beams east so that they lay along the collimator axis.

The beam transverse profile was monitored at several points in the beamline by Segmented Wire Ion Chambers (SWIC). These profiles could be characterized, assuming Gaussian distributions, by the standard-deviation in $\mathrm{x}\left(\sigma_{x}\right)$ and in $\mathrm{y}\left(\sigma_{y}\right)$. At the entrance to the collimator, the East beam typically had $\sigma_{x}=0.7 \mathrm{~mm}, \sigma_{y}=1.0 \mathrm{~mm}$, the West beam had $\sigma_{x}=1.0 \mathrm{~mm}, \sigma_{y}=1.5 \mathrm{~mm}$. The combined intensity of both beams was measured by a Secondary Emission Monitor (SEM) located before the split. The intensity of each beam was not measured directly, but instead inferred their relative intensity from event rates in the spectrometer.

\subsection{Targets and Neutral Beam Channel}

Each of the two proton beams interacted in one of two targets upstream of the collimator to produce two neutral beams. Figure 2.2 shows the relative orientation of the targets, and Table 2.1 their physical dimensions. The smaller Downstream (DS) target was located just inside the collimator and the sweeper magnet M1. The larger Upstream (US) target was located $2545.6 \mathrm{~cm}$ upstream of the other target. The relative intensities of the US and DS target proton beams was $\sim 20: 1$. This split was adjusted so that the spectrometer observed equal numbers of $K_{L}^{0}$ decays from each target. The targets were made of Hevimet, an alloy of $98 \%$ Tungsten, $2 \%$ Copper. Tungsten's short $(0.38 \mathrm{~cm})$ radiation length reduced the number of high energy photons escaping from the target which could become unwanted background events in the spectrometer. Each target was 


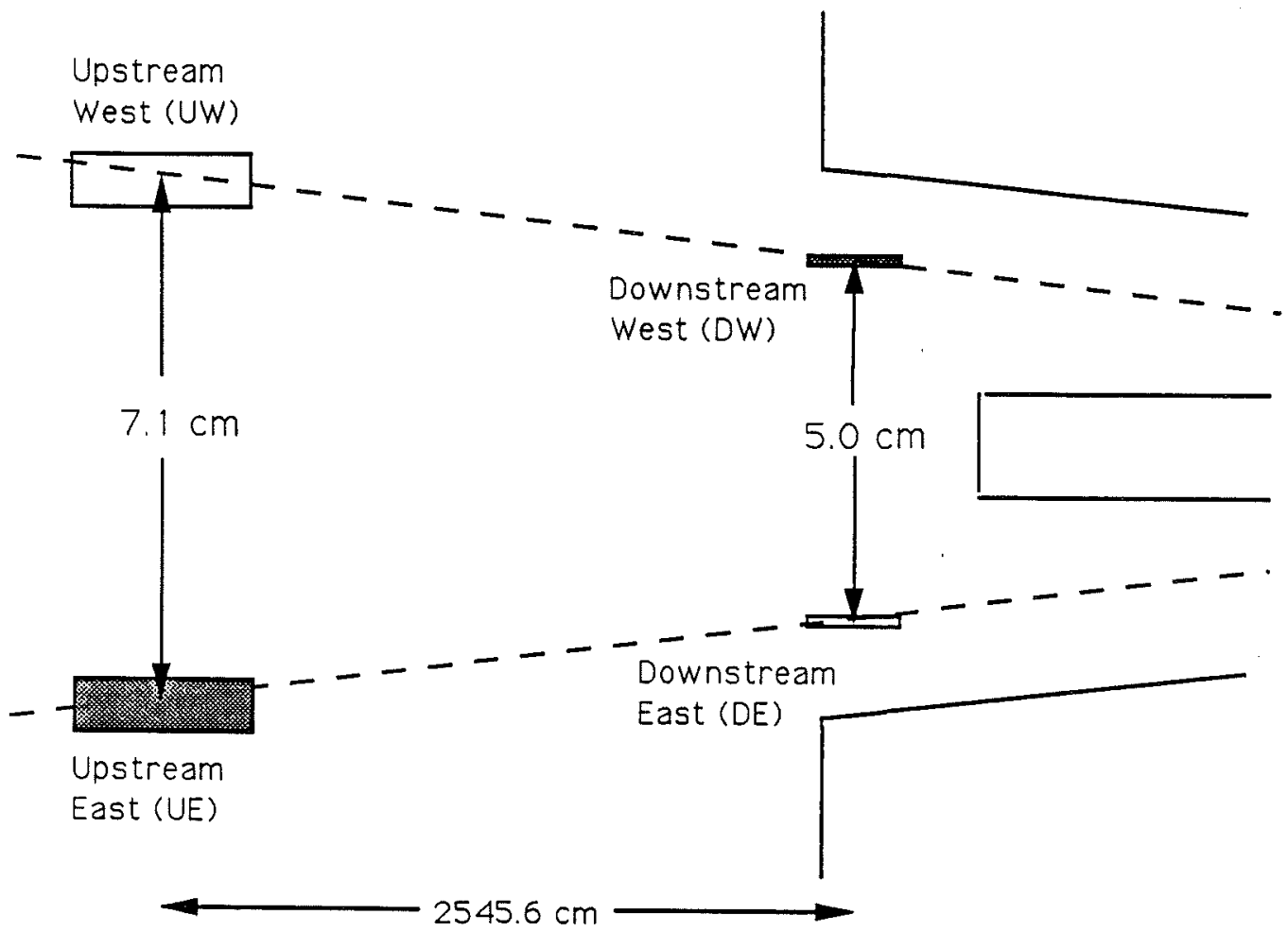

Figure 2.2: E621 target area

\begin{tabular}{|l|cc|}
\hline Parameter & DS Target & US Target \\
\hline Z position $(\mathrm{cm})$ & -726.4 & $\mathbf{- 3 2 7 2 . 0}$ \\
Material & $98 \%$ Tungsten & $98 \%$ Tungsten \\
Length $(\mathrm{cm})$ & 9.58 & 19.09 \\
Interaction lengths & 1 & 2 \\
Cross-section & circle & square \\
- dimensions $(\mathrm{cm})$ & diameter 0.35 & side 1.26 \\
\hline
\end{tabular}

Table 2.1: Target parameters 
attached to a motor drive for precise lateral positioning. Typically one target intersected the East beam while the other intersected the West beam. The exact lateral positions of the targets were adjusted so that the neutral beams produced intersected at the rear of the spectrometer. On a regular basis, the targets were moved so that they switched beams. This was done to reduce any systematic effects. This target switching required moving the septum vertically to change the fraction of the beam on each target, as well as adjusting the Lambertson and dipole magnets field strengths, but required no change in the focussing elements.

The collimator channels, detailed in Figure 2.3, defined the neutral particle beams seen by the spectrometer. The 7.31 meter long collimator channel was located in the middle of the M1 sweeper magnet. This magnet was operated at a current of 3600 Amps, producing a field of 35.72 kilogauss $(\mathrm{kG})$ with an integrated B·d $\ell$ of $261.1 \mathrm{kG}-\mathrm{m}$. This field swept charged particles out of the neutral beam channel. The polarity of this magnet was reversed on a regular basis to reduce systematic effects.

The collimator was formed by a series of brass blocks, each of which had a circular hole drilled through it to create the channel for the neutral beam. The collimator had three major sections. The first section was $390 \mathrm{~cm}$ long, with the diameter of the holes forming the channel decreasing along the section. This section was long enough that even $800 \mathrm{GeV}$ beam energy protons would be swept by the magnetic field into the wall of the channel upstream of the end of the section and thus be kept out of the spectrometer. The middle section had an neutral beam-defining aperture $0.32 \mathrm{~cm}$ in diameter. The holes in each block of this section subtended the same transverse angle (0.78 mrad) from a point on the DS target. This minimized the scattering of neutral beam particles in the channel. The channel of the defining section was lined with tungsten to increase the absorption of stray photons. In the last section the channel increased in diameter to keep material away from the neutral beam near the end of the collimator, where charged particles produced by scattering of beam particles might not be swept away 

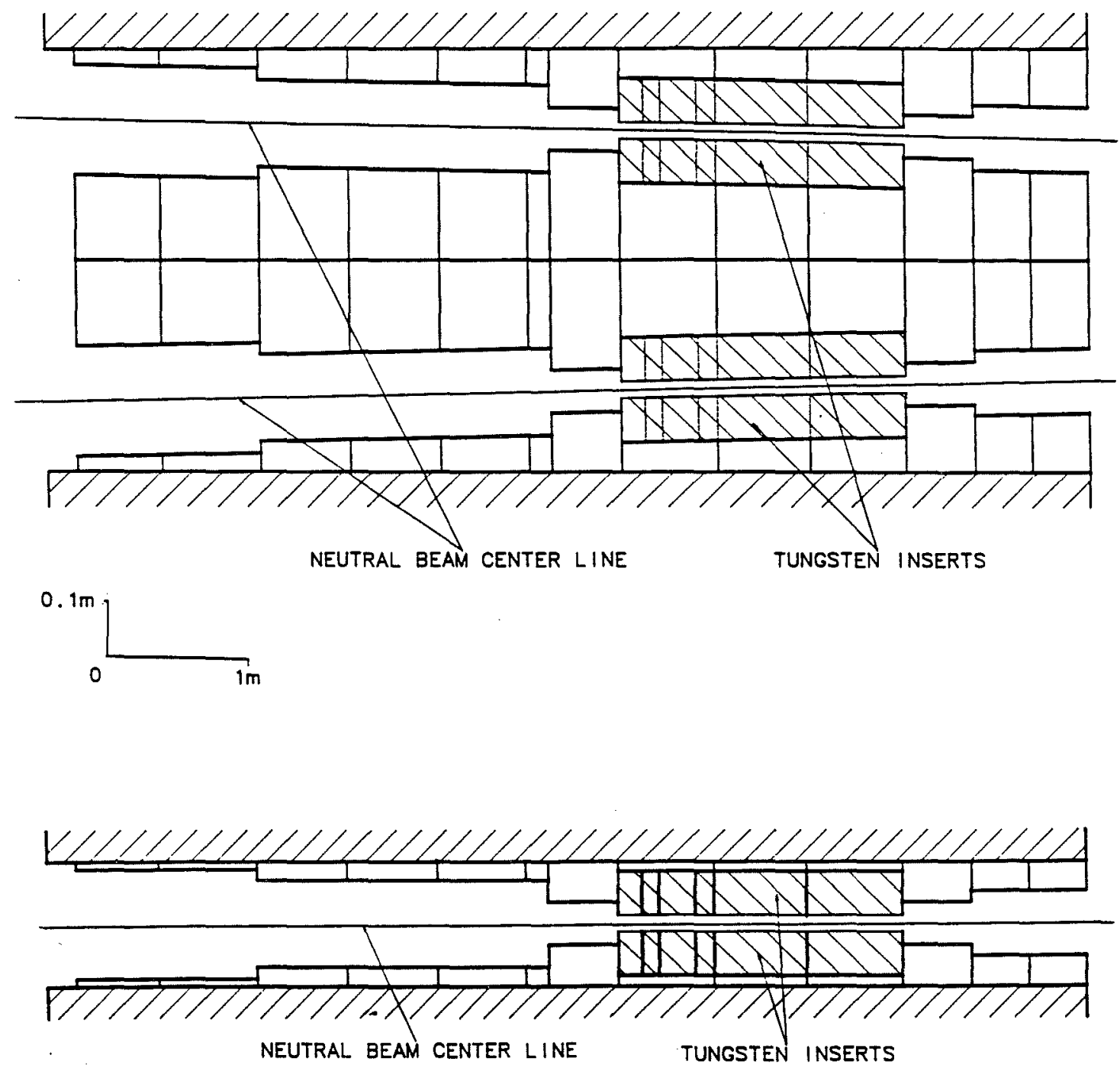

Figure 2.3: E621 neutral beam collimator 
by the magnetic field. The East and West collimator channels were convergent in the horizontal plane, with a relative angle of $0.8 \mathrm{mrad}$. The defining apertures of the two channels had a transverse separation of $4.74 \mathrm{~cm}$ center-to-center to enable the event reconstruction to identify the production target of each decaying neutral beam particle.

The neutral beams had only a small $(<1 \mathrm{mrad})$ angle relative to the proton beams which produced them. This minimized any differences in production between the DS and US targets and maximized the ratio of baryons to photons in each neutral beam. Photons and neutrons in the neutral beams formed unwanted background events in our spectrometer when they interacted with some material, producing secondary charged particles. The material in the region of the neutral beams, such as beampipe windows and air molecules, was minimized by making the beamline a continuous evacuated space from just downstream of the US target, through the collimator channel, and into the decay volume of the spectrometer.

\subsection{Spectrometer}

Figure 2.4 is a plan view of the spectrometer. The spectrometer had a vacuum chamber in which the neutral particle decays occurred. It also had trigger counters, wire chambers to measure charged particle positions, momentum analyzing magnets, and a segmented lead glass calorimeter to measure the energy and position of photons.

The $z$ axis of the coordinate system was along the West neutral beam channel. This was defined by the trajectory of a low-intensity proton beam used to align the chambers (See Section 2.8.1). The origin of the $z$ axis was at the downstream face of the sweeper magnet. The $-z$ direction was towards the accelerator (upstream), while $+z$ was towards the spectrometer (downstream). A right-hand coordinate system was used, with $+x$ towards the west (to the left looking downstream) and $+y$ being up. The $z$ positions of the spectrometer elements are listed in Table 2.2. 


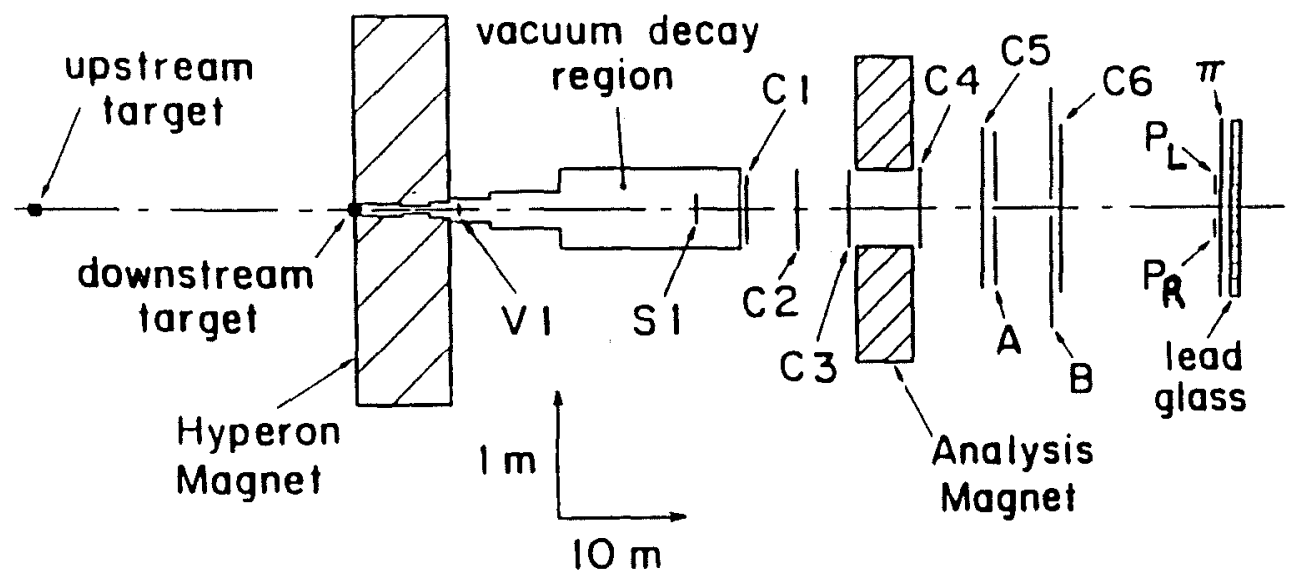

Figure 2.4: The E621 spectrometer

\begin{tabular}{|l|c|}
\hline Element & Z-Position $(\mathrm{cm})$ \\
\hline V1 Counter & 86.5 \\
DK Counter & 1949.8 \\
Decay Pipe Window & 2258.2 \\
Chamber C1 & 2322.4 \\
Chamber C2 & 2689.4 \\
Chamber C3 & 3053.3 \\
Magnet M2 & 3236.3 \\
Magnet M3 & 3465.5 \\
Chamber C4 & 3661.3 \\
Chamber C5 & 4165.5 \\
A Hodoscope & 4232.4 \\
B Hodoscope & 4737.7 \\
Chamber C6 & 4819.9 \\
$\pi, p$, V2 Counters & 6014.2 \\
Face of Lead Glass Array & 6092.2 \\
\hline
\end{tabular}

Table 2.2: Z-positions of the spectrometer elements. The origin of the $\mathrm{z}$-axis was at the downstream end of the collimator. 


\subsubsection{Decay Volume}

Neutral particle decays were accepted if they occurred in the $1863 \mathrm{~cm}$ long space between veto counter V1 and decay trigger counter DK. These counters each consisted of $1 \mathrm{~mm}$ thick plastic scintillator wrapped in $0.25 \mathrm{mil}(.006 \mathrm{~mm})$ aluminized mylar. Each was connected via a clear plastic "light pipe" to a $5 \mathrm{~cm}$ diameter photo-multiplier tube (RCA 8575). The scintillator was inside an evacuated pipe which was $10 \mathrm{~cm}$ in diameter near V1, increasing to a diameter of $61 \mathrm{~cm}$ at the DK counter. The vacuum ended in a thin 5 mil $(0.127 \mathrm{~mm})$ mylar/sailcloth window after the DK counter.

\subsubsection{Charged-Particle Spectrometer}

Six Multiple Wire Proportional Chambers (MWPC's) measured the position of charged particles passing through them. Labelled as C1-C6 in Figure 2.4, three were located in front of momentum-analyzing magnets M2 and M3, and three after them. These chambers had been used in several previous experiments[17,18]. The particular chambers used and their relative positions was the same as that previously used during a 1984 experiment[19].

Each chamber was a sandwich of 5 wire planes, each plane mounted on $0.5 \mathrm{~cm}$ thick G-10 fiberglass board. Three of the planes were high-voltage planes, wound with $2.5 \mathrm{mil}$ $(0.064 \mathrm{~mm})$ diameter copper-beryllium wire on $1 \mathrm{~mm}$ centers and typically held at a potential of $-3.0 \mathrm{kV}$. (Chamber $2 \mathrm{had}$ been rewound with $3.0 \mathrm{mil}$ wire.) The others were a pair of signal planes, wired orthogonally to each other with 1.0 mil $(0.025 \mathrm{~mm})$ diameter gold-plated tungsten wire on $2 \mathrm{~mm}$ centers. The signal plane orientations were $x$ and $y$, except for chamber 2 which was rotated $45^{\circ}$ to provide so-called $u$ and $v$ planes. Data from this chamber resolved ambiguities in matching up tracks in the $x$ planes with those in $y$. Each chamber was sealed with $0.5 \mathrm{mil}(0.013 \mathrm{~mm})$ mylar windows and filled with gas at just above atmospheric pressure. The gas was a mixture of Argon and Freon B1, bubbled through a bottle of methylal kept near $0^{\circ} \mathrm{C}$ by an 
ice-water bath. The resulting fractions by volume were typically Argon 94.4\%, Methylal $5.5 \%$, and Freon $\mathrm{B} 10.1 \%$.

Analyzing magnets M2 and M3 provided nearly uniform vertical magnetic fields which bent the charged particles in the $x-z$ plane to allow a determination of their momentum. Each magnet was 2.29 meters long with 1.83 meter long poles. M2 had an $\mathrm{x}$-y aperture of $25.4 \times 61.0 \mathrm{~cm}^{2}$, and M3 an aperture of $30.5 \times 61.0 \mathrm{~cm}^{2}$. Both M2 and M3 were operated at a current of 2500 Amps, which produced fields of $16.1 \mathrm{kG}$ and $11.5 \mathrm{kG}$ respectively, resulting in an effective combined transverse momentum $\left(P_{T}\right)$ "kick" of $1.55 \mathrm{GeV} / \mathrm{c}$. The polarity of these fields was reversed regularly to reduce systematic errors. The chambers and other spectrometer elements were aligned symmetrically in $x$ about the centerline of the magnets so that the acceptance would remain essentially the same when the fields were reversed.

Between chambers 5 and 6 there were two planes of counters, termed the A and B hodoscopes, which were 3 meters apart in $\mathrm{z}$. These were used to provide "fast" signals to trigger on the charged particles from the decays. Each was divided in $\mathrm{x}$ into several elements to allow triggering based on the momentum of the charged particles. Each element was a $5.72 \mathrm{~cm}$ wide by $0.48 \mathrm{~cm}$ thick piece of plastic scintillator, $40.64 \mathrm{~cm}$ high in the A hodoscope and $60.96 \mathrm{~cm}$ high in the $\mathrm{B}$ hodoscope. The elements were overlapped in the $\mathrm{x}$ view, and their centers were $5.08 \mathrm{~cm}$ apart. Each was wrapped in aluminum foil, opaque paper and tape, and connected to a photo-multiplier tube. The A hodoscope had 22 elements, with 11 elements on a side, and the B hodoscope had 28 elements, 14 on a side. Each hodoscope had a two-element gap $(9.53 \mathrm{~cm})$ between the left and right sides to allow for the passage of non-decaying neutral beam particles.

Between the chambers, inside the spectrometer magnet apertures and between chamber 6 and the lead glass were polyethylene bags filled with helium at just above atmospheric pressure. This reduced the amount of material in region of the neutral beam. The windows of the bags were $1 \mathrm{mil}(0.0254 \mathrm{~mm})$ thick. The spectrometer as a whole 
had 0.05 radiation lengths of material in the region of the beam.

\subsubsection{Photon Detector}

An array of 86 lead glass blocks, stacked in seven rows, was used to detect the pair of photons from the $\pi^{0}$ decay. Each block was $10 \times 10 \times 38.4 \mathrm{~cm}^{3}$, arranged with the long axis parallel to the beam. They were made of type SF2 lead glass, which has a radiation length of $3.2 \mathrm{~cm}$. Each row was staggered a half-block from the adjacent ones, so each block had only six nearest neighbors. A block was left out of the array in the center as the afore-mentioned "hole" to allow the neutral beam particles to pass through without showering. This arrangement is shown in Figure 2.5, along with the veto and trigger counters just upstream of the array. Each block was wrapped in foil, paper and tape, and had a plastic fixture attached to the downstream end with double-sided adhesive tape. This tape actually provided a more long-lasting attachment than the epoxy we had used previously. A $5 \mathrm{~cm}$ diameter photo-multiplier tube (RCA 6342A/V1) with a threaded plastic collar was screwed into a tapped hole in the fixture. This kept the tube in contact with the face of the block, using optical grease for the coupling. The different high voltages (HV) needed by the stages of the tubes to amplify the signals were provided by a resistor network in the bases connected to the end of the tubes. These bases provided the output signals from the tubes to external coaxial cables to be transmitted to the data acquisition electronics. The bases were typically operated at $-1400 \mathrm{~V}$.

These lead glass blocks had a depth of 12 radiation lengths, which was insufficient to contain all the electromagnetic shower energy, especially for higher energy photons. To improve the energy and position resolution for the photons, a pre-converter made up of lead sheets totalling $0.75 \mathrm{in}(1.91 \mathrm{~cm})$ in thickness were placed on the face of the array for most of the experiment. This pre-converter added 3 radiation lengths to the depth of the array, for a total of 15 . As this added volume was inactive, it did reduce the 


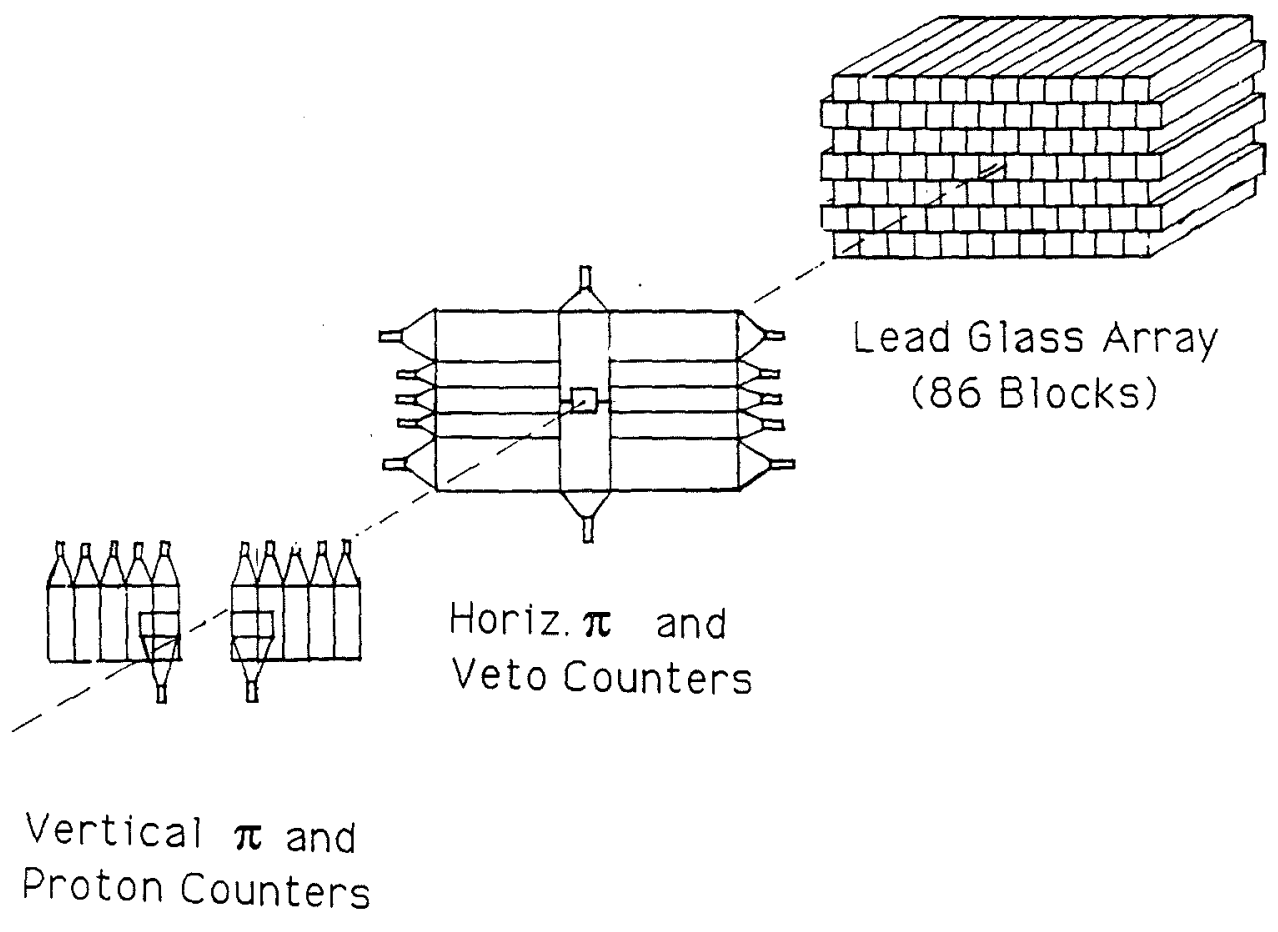

Figure 2.5: Lead glass array and veto counters 


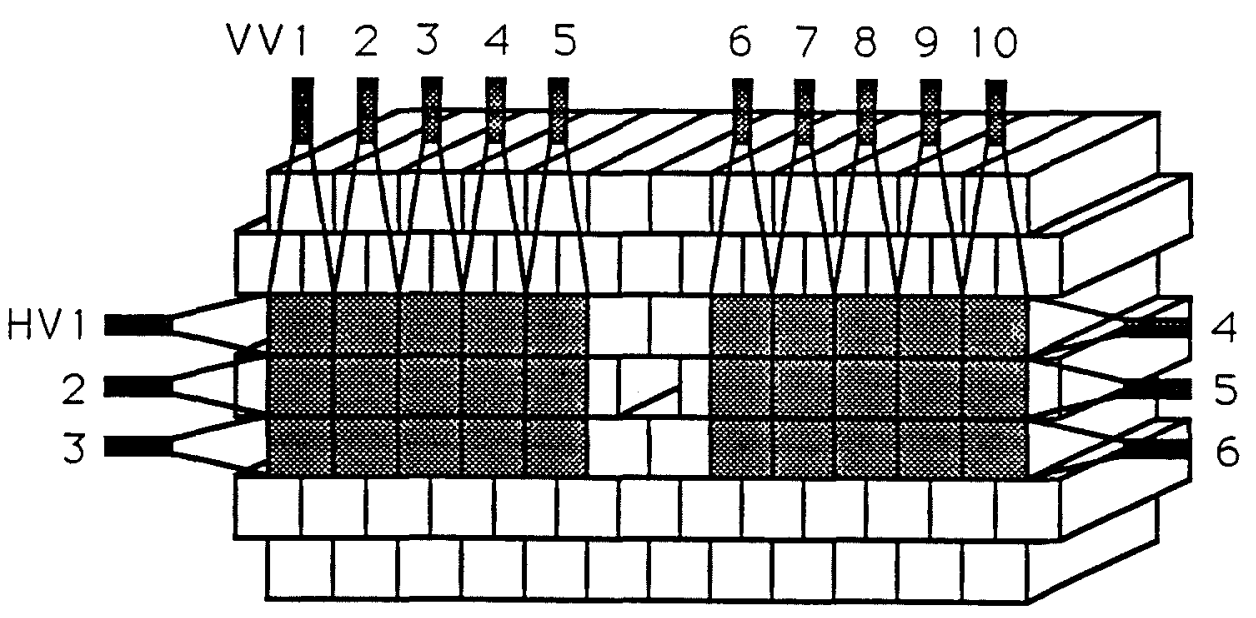

Figure 2.6: Overlapped $\pi$-killer counters relative to the lead glass array. There were 6 horizontal counters (HV1-6) and 10 vertical ones (VV1-10).

response for low-energy $(<10 \mathrm{GeV})$ photons. By initiating showers earlier in the array, it improved the energy resolution for high energy photons. The pre-converter reduced the loss of shower energy for photons which passed close to the cracks between blocks. This was because the shower tended to start in the lead, whereas without the lead sheets, the photons had a greater chance of passing down the cracks between the blocks and not starting a shower until a good distance into the array. The showers which started early tended to spread more transversely, which improved the measurement of their position in the array.

To veto charged particles which might cause showers in the array, two sets of counters were used. In the central region, where the charged particles could be from decay products in an event, a " $\pi$-killer" veto constructed of 16 overlapping horizontal and vertical counters (Figure 2.6) was used. A hit in an overlapped pair of these counters, which were similar in construction to the A and B hodoscope counters, served to remove a group of blocks behind the overlapped section from the $\gamma$ trigger. This is described in Section 2.6 below. The rest of the array was covered by six larger counters. A hit in 
any of these counters, which would not be from a valid decay product, vetoed events from the $\Xi^{0}$ trigger. Mounted in front of the $\pi$-killers were two small trigger counters, termed the proton counters $P_{L}$ and $P_{R}$. They were each $8 \times 4 \mathrm{in}^{2}\left(20.3 \times 10.2 \mathrm{~cm}^{2}\right)$ and mounted $2 \mathrm{in}(5.1 \mathrm{~cm})$ to either side of the hole to avoid overlapping the VT and VB counters. These counters were to allow events to be seen which had high-energy protons that missed the $A$ and $B$ hodoscopes. All of the above counters were located 1 meter in front of the lead glass array to reduce spurious vetoes due to backscatter from photon showers.

\subsection{Trigger Logic}

The trigger logic determined when it was appropriate to digitize and read out the detector information to capture a particle decay event. More than one set of electronic logic, each termed a trigger, was used at a time. Figure 2.7 shows how all the triggers used in the experiment were connected. Because the less selective triggers occurred at a rate that would overwhelm the data acquisition system, their outputs were passed through "prescalers". These units would only put out a one pulse for every $2^{\text {n }}$ input pulses, where $n$ is an integer. The only trigger not passed through a prescaler was the $K_{\pi 3}$ trigger, which had the lowest rate but made up the majority of the triggers written to tape.

\subsubsection{Approach}

The design of the $\Lambda$ and $\Xi^{0}$ decay triggers was influenced by how they would be used. For most of the data-taking period, these triggers were used parasitically. This meant that they were used at the same time as the main E621 triggers, but were restricted by prescalers to make up only $\sim 10 \%$ of all triggers written onto tape. The performance of our chambers limited the proton beam intensity that could be efficiently used, while the total number of triggers which could be written to tape during a spill was limited 


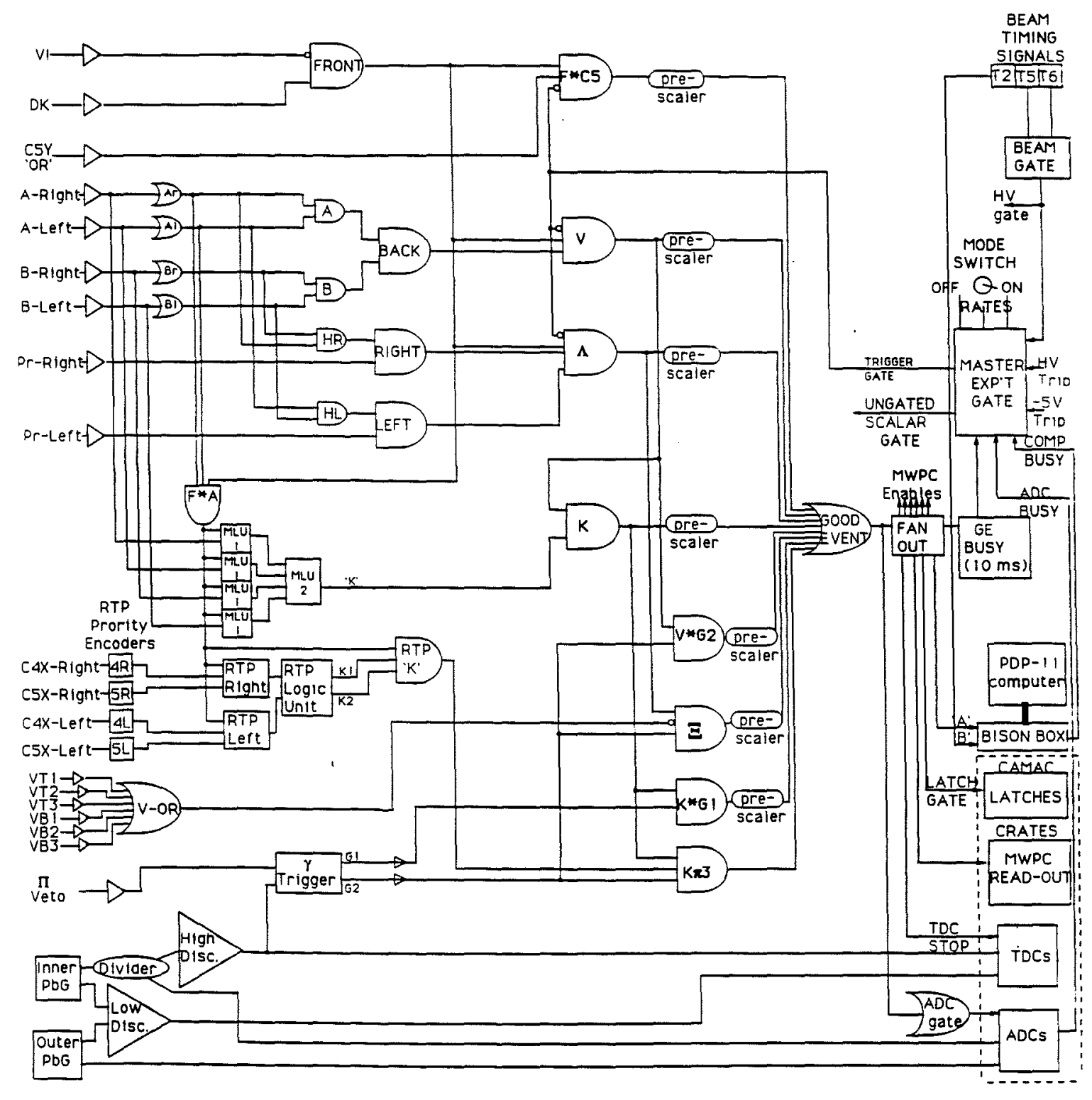

Figure 2.7: E621 trigger diagram 
by the data acquisition system. Hence, the $\Lambda$ and $\Xi^{0}$ triggers had to have as high a yield of reconstructable decays as possible. The $\Lambda$ and $\Xi^{0}$ triggers were arranged in a hierarchy of increasing complexity, so that the biases created in trying to achieve good yields could be studied at each level

Referring to Figure 2.7, the triggers used were the F.C5 , $\Lambda$, and $\Xi^{0}$ triggers. The F.C5 trigger was the least restrictive and events reconstructed from this sample were used to determine the efficiencies of the downstream trigger counters in the other triggers. The $\Lambda$ decay events from the F.C5 trigger were used to find the $\Lambda$ lifetime. The $\Lambda$ trigger, yielding mostly $\Lambda$ decays, had the same charged-particle trigger elements as the $\Xi^{0}$ trigger, but without using the $\gamma$ trigger. The $\Lambda$ decay events from the $\Lambda$ trigger were also used for lifetime measurements. The $\Xi^{0}$ trigger was the most restrictive, and provided the $\Xi^{0}$ event sample. Each of these triggers is presented in greater detail below.

\subsubsection{The F.C5 trigger}

This trigger started with the same basic "neutral decay" logic as the others. This was a signal in the DK counter without a corresponding signal from the V1 veto counter. This signified that a neutral particle had passed into the decay volume and subsequently decayed into one or more charged particles. This was the FRONT (F) part of the trigger. The other requirement was a signal from the logical OR of all the horizontal (or $y$-view) wires in Chamber 5, which indicated at least one charged particle was of high enough energy to pass through the field of the analyzing magnets. The full logical expression for the trigger was $D K \cdot \overline{V 1} \cdot C 5_{Y}$. This trigger was relatively unbiased, reflecting only the basic geometric constraints of the apparatus. It had a yield of $18 \%$ fully-reconstructed $\Lambda$ decays. 


\subsubsection{The $\Lambda$ trigger}

This trigger was designed to improve on the yield of the F.C5 trigger. It required two tracks of opposite charge downstream of the analyzing magnets, which had deflected then to opposite sides of the spectrometer. The logic for the $\Lambda$ trigger was $D K \cdot \overline{V 1}$. $L E F T \cdot R I G H T$, where $\operatorname{LEFT}(L)$ and $R I G H T(R)$ meant a track on that side of the apparatus downstream of the analyzing magnets. $L E F T$ was defined as $\left(A_{L} \cdot B_{L}\right)+P_{L}$, RIGHT as $\left(A_{R} \cdot B_{R}\right)+P_{R}$. $A_{L}$ (or $A_{R}$ ) was the logical OR of all the elements on the left (or right) side of the A hodoscope, similarly for $B_{L}, B_{R}$ and the $B$ hodoscope. The $A$ and $B$ hodoscope signals were used in coincidence to reduce backgrounds due to noise in the separate counter elements. The proton has a rest mass (938 MeV) six times greater than that of the $\pi^{-}(140 \mathrm{MeV})$. Thus for high-energy $\Lambda$ 's $(>7 \mathrm{GeV})$, the decay of the $\Lambda$ is very asymmetric in terms of lab momentum, with the proton being at least three times as energetic as the $\pi^{-}$. To increase the acceptance for high-energy protons which were deflected very little by the magnets, the proton counters $P_{L}, P_{R}$ located near the lead glass were added into the trigger. This trigger had a yield of $37 \%$ reconstructed $\Lambda$ decays.

The $\Lambda$ trigger did introduce some biases in the selection of events. The inner edges of the proton counters, being $4 \mathrm{in}(10 \mathrm{~cm})$ from the hole center so as not to overlap the lead glass vetoes, were not close enough to the neutral beams to catch all the protons. The most important events for a lifetime measurement are those at lower momentum, where the protons should not approach the inner edges of these counters. This placement of the proton counters was thus thought to be an acceptable compromise within the constraints of the apparatus. The main background to the $\Lambda$ events were the $K^{0} \rightarrow \pi^{+} \pi^{-}$decays. They were less numerous than the $\Lambda$ 's, and could be separated, in most cases, from the $\Lambda$ 's by the reconstruction. They were not a background for the $\Xi^{0}$ 's as they lacked a pi-zero. Thus it was not necessary to remove them at the trigger level. By not optimizing the $\Lambda$ trigger against the $K_{S}^{0}$ events, it could be kept simple 
and incur no additional bias.

The trigger symmetry in $\mathrm{x}$ meant that the acceptance was nearly identical for $\Lambda$ 's, and their anti-particles $\bar{\Lambda}$ 's, which decay as $\bar{\Lambda} \rightarrow \bar{p} \pi^{+}$and thus also for $\Xi^{0}$ 's and $\overline{\Xi^{0}}$ 's. Thus the anti-particle decay events were collected at the same time as the more copious particles, and had the same acceptances.

\subsubsection{The $\Xi^{0}$ trigger}

Topologically, a $\Xi^{0}$ decay is a $\Lambda$ with two $\gamma^{\prime}$ s from the $\pi^{0}$. The photon trigger described below provided a signal, G2, if there were at least two showers in the lead glass array not vetoed by hits in the $\pi$-killer counters. The other six veto counters, VT1,2,3 and VB1,2,3, covered the rest of the array and were used to veto events with spurious charged particles. The logical oR of the six veto counter outputs $\left(V_{O R}\right)$ was formed. A $\Xi^{0}$ trigger which rejected events with a $V_{O R}$ signal had a $7 \%$ yield for fully reconstructed $\Xi^{0}$ decays, which was three times greater than the $2.5 \%$ yield from a $\Xi^{0}$ trigger requiring

just the $\Lambda$ and G2 signals. To take advantage of this improved yield, the $\Xi^{0}$ trigger was made $\Lambda \cdot G 2 \cdot \overline{V_{O R}}$. This trigger, like the two $\Lambda$ triggers, was prescaled as otherwise it would have overwhelmed the $K_{\pi 3}$ trigger, for which $\Xi^{0}$ decays were a background. The details of the $\gamma$ trigger electronics are presented in Appendix A.

\subsection{The $\gamma$ Trigger}

The $\gamma$, or photon, trigger was designed to determine that there were at least two showers of energy in the lead glass blocks which were coincident with a neutral decay trigger and not associated with hits in the $\pi$-killer. The $\gamma$ trigger had to make its decision in $<100 \mathrm{~ns}$, so as not to adversely affect the trigger rate and data acquisition rate. The timing of the showers had to be as tight as feasible to help reject the main background of $\Lambda$ decays with two unassociated random photon showers. The trigger hardware was 


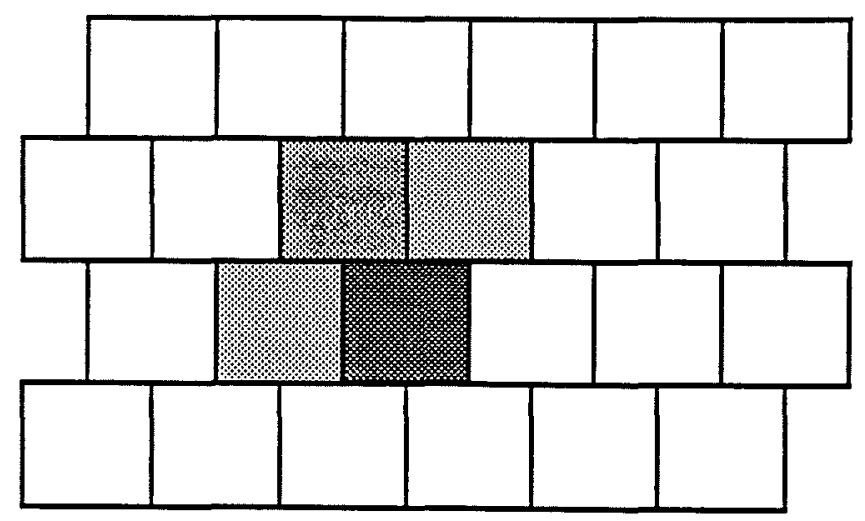

Figure 2.8: Example cluster of lead glass blocks with signals due to an electromagnetic shower. The shading indicates the relative magnitudes of the signals from the photo-multiplier tubes on each block.

kept relatively simple, in order to improve its chances of remaining reliable over the eight months of the experiment.

An electromagnetic shower in the lead glass array typically spread energy over a few blocks (Figure 2.8). Any contiguous group of blocks which each had an output signal from the its photomultiplier tube above a threshold was termed a "cluster". The trigger counted how many such clusters there were in the array.

The trigger needed to ensure that the clusters it counted were not overlapping, which could happen if the photons hit the array too close together. This was because such overlapping showers could not be separated by the reconstruction software with any degree of certainty.

\subsubsection{Cluster-Counting Algorithm}

The first step in identifying clusters was to pass a fraction of each block's photo-tube output through a discriminator which was set at a relatively high level equivalent to $\sim 2 \mathrm{GeV}$ of electromagnetic energy. This rejected small pulses due to noise and small 
Nearest- Neighbors of Block 'B':

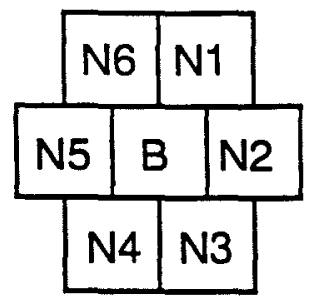

\section{Cluster-Edge Algorithm :}

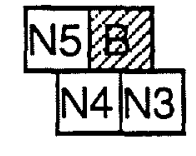

Pattern

\section{$E D G E=B * \bar{N} 3 * N 4 * \overline{N 5}$}

Logic

Figure 2.9: Example of a cluster edge-finding algorithm.

random photons, which could have confused the trigger. It also avoided rejecting clusters which were separated by a block with a signal too small to affect each cluster's energy resolution. The length of the cables from each block to the discriminator were adjusted so that the variation in the arrival times of the leading edges of all the incoming pulses from separate blocks of the array in an event was less than $5 \mathrm{~ns}$.

Cluster identification involved the novel use of an image processing technique called an "edge-finding algorithm". This technique takes advantage of the fact that a cluster is defined not so much by its size as by its edges, where it is surrounded by blocks which have no signals. Determining if a block was an "edge" of a cluster only required comparing it to some of its neighboring blocks. Figure 2.9 shows such an edge-finding algorithm. In the E621 lead glass array, each block had six "nearest-neighbors", which have been labelled in clockwise order N1-N6. In the example of Figure 2.9, the logical definition of "EDGE" was

$$
E D G E=B \cdot \overline{N 3} \cdot \overline{N 4} \cdot \overline{N 5}
$$


Cluster-Edge Algorithm Pattern : $\begin{array}{ll}\mathrm{N5} / \mathrm{g} \\ \mathrm{N} 4 \mathrm{~N} 3 \\ \mathrm{~N}\end{array}$ Application of Algorithm to Three Example Clusters :

(a)

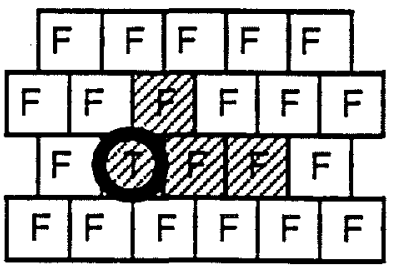

No. of Edges: 1 (b)

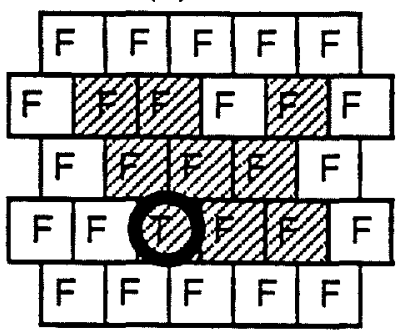

1 (c)

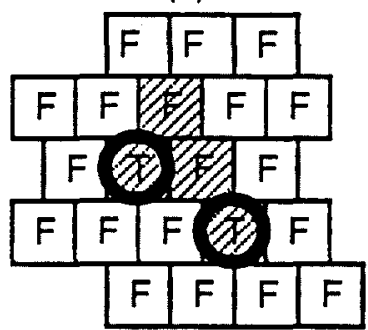

2

Figure 2.10: Application of edge-finding algorithm on three example clusters, labelled (a),(b) and (c).

Here, $B$ denotes a signal in the block and $\overline{N 3}, \overline{N 4}, \overline{N 5}$ the absence of signals from those three neighboring blocks. Figure 2.10 shows the application of this particular edgefinding algorithm to three example clusters. Applying the algorithm to each block results in only one TRUE(T) output for clusters (a) and (b). Thus, the algorithm not only signals that a cluster is present, it usually has only a single TRUE output per cluster. If any cluster in the array had only one TRUE edge output, then a simple sum of the outputs would determine the number of clusters. As can be seen in Figure 2.10(c), however, some clusters had two "edges" which satisfy the algorithm. To correct for this, three edge algorithms were implemented in parallel. Of the six possible edge algorithms, the ones shown in Figure 2.11 were chosen. The final number of clusters was determined by the minimum of the output of the three separate algorithms. This three algorithm method correctly determined the number of cluster in all but some 


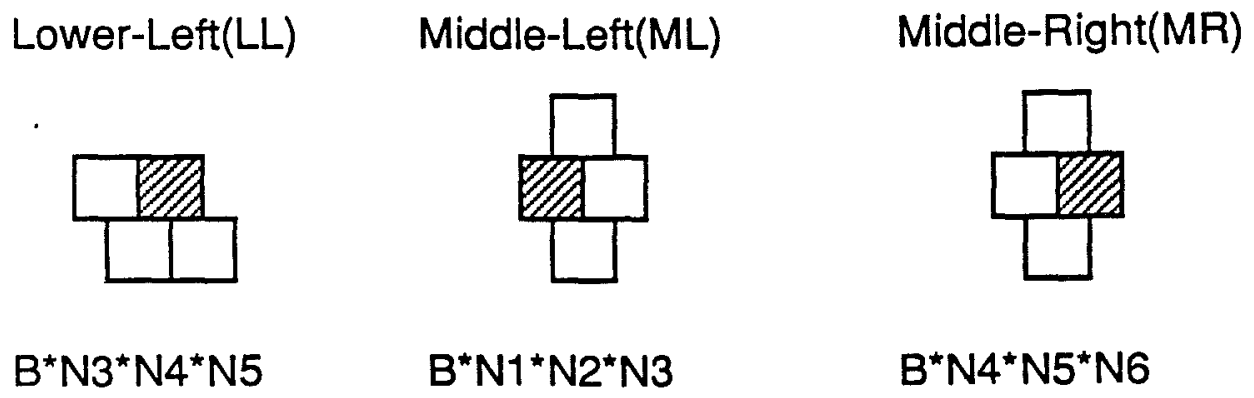

No. of Clusters $=$ Minimum(LL"ML*MR)

Figure 2.11: Edge-finding algorithms used in the $\gamma$ trigger

very extended clusters. The method was quite adequate for counting electromagnetic showers.

\subsection{2 $\pi$-Killer Vetoes}

As mentioned above, the $\pi$-killer counters were used to reject clusters caused by charged particles interacting in the lead glass array. The outputs of the 16 counters, 10 vertical and 6 horizontal (See Figure 2.6 above), were sent sent to discriminators $(25 \mathrm{mV}$ threshold) and the resulting logic signals (60 ns width) sent to the photon trigger. If a pair of overlapping (horizontal and vertical) counters both had signals, then veto signals were generated to prevent any signals from several underlying blocks from reaching the cluster-counting logic. As shown in Figure 2.12, if the two counters crossed over a block, then seven blocks were vetoed out of the trigger. If they crossed between two blocks, then a pattern of eight blocks was vetoed. 

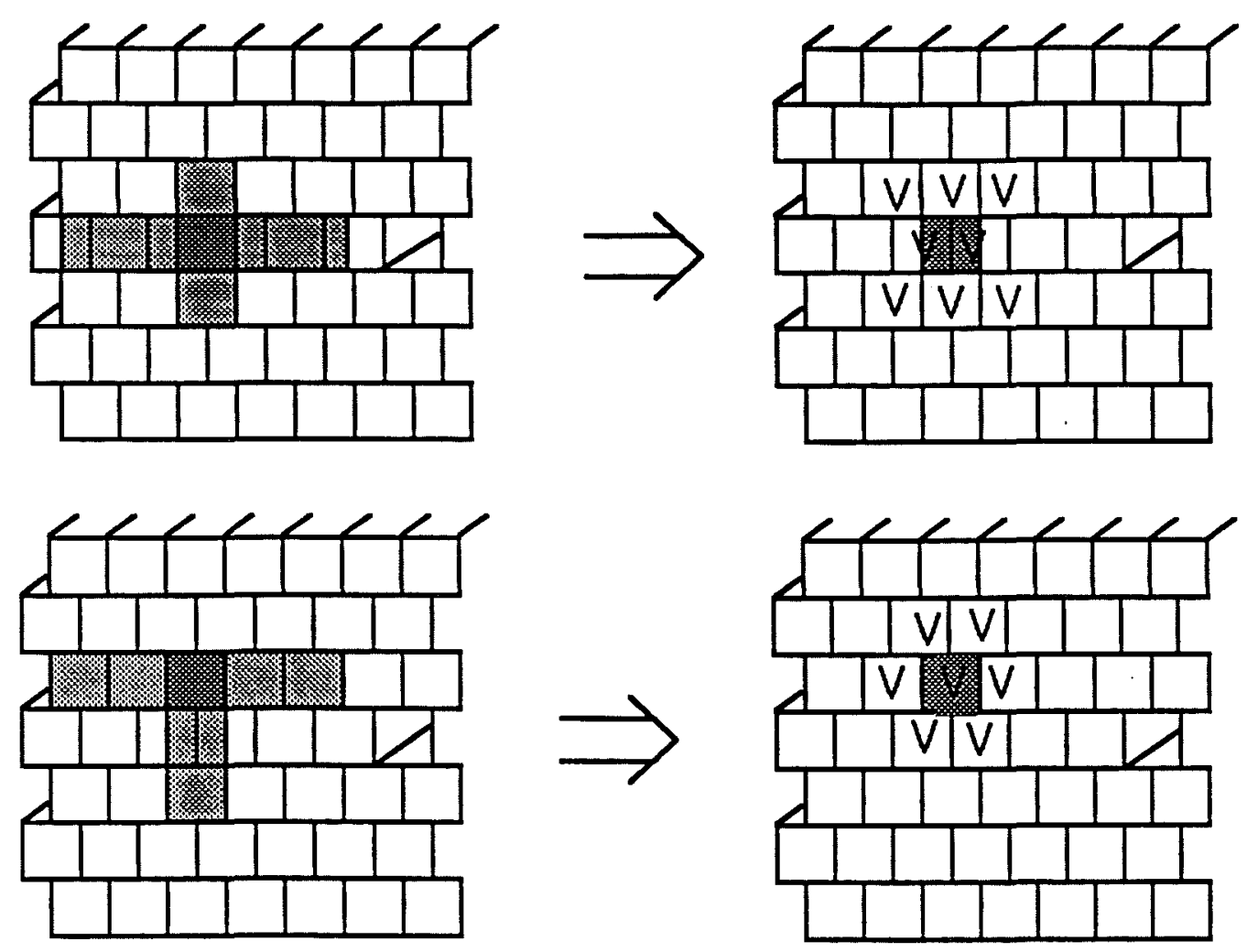

Figure 2.12: Blocks in $\gamma$ trigger vetoed by hits in the $\pi$-killer array. The first diagram shows that if hit horizontal and vertical counters overlapped between two blocks, then 8 adjacent blocks were removed from the trigger. The second diagram shows that if the overlap was centered on a block, then 7 blocks were vetoed from the trigger 


\subsubsection{Implementation}

To provide signals for the data acquisition and photon trigger, two anode outputs from the high-voltage splitter bases of the photomultiplier tubes were used. As shown in Figure 2.7, one output went directly to discriminators in the experimental hall (LeCroy 4416's), with low $15 \mathrm{mV}$ thresholds ( $0.4 \mathrm{GeV}$ ), whose outputs were sent by cables to a set of Time-to-Digital Converters(TDC's) in the trailers. The 34 blocks which were on the outside of the array were not used in the trigger because a shower centered in them was likely not to be fully contained by the array and thus would be reconstructed very poorly. These outer blocks had their other output sent directly to the Analog-to-Digital Converters(ADC's) in the trailer. For the 52 inner blocks, this output was unevenly split by a resistor triad. The majority of the signal went to the a set of discriminators with higher $25 \mathrm{mV}$ thresholds ( $\sim 2 \mathrm{GeV}$ ), whose outputs (20 ns width) went to both the glass trigger and another set of TDC's. These TDC's were used to verify that the $\gamma$ trigger inputs for different blocks had the same relative timing and to check the operation of the trigger.

\subsubsection{Performance}

The important performance measure for the $\gamma$ trigger was the efficiency that it had an output of ' $\geq 2$ ' clusters for events with 2 photon clusters in the array. To measure the efficiency, event were used which had satisfied the ' $K$ ' trigger, which had no requirement from the $\gamma$ trigger (See Figure 2.7). First, events which had 2 photon clusters, and for which a software simulation of the $\gamma$ trigger counted at least 2 clusters were analyzed. For the 2 photon events, the $\gamma$ trigger had a latched output of ' $\geq 2$ ' $85 \%$ of the time, and ' $\geq 1$ ' the remaining $15 \%$. Next, events with 1 photon cluster and an answer of ' $\geq 1$ ' from the simulator were studied. For these 1 photon events, the $\gamma$ trigger output ' $\geq 1$ ' $95 \%$ of the time, ' $\geq 2$ for $1.5 \%$ of the events, and 0 for the remainder. 


\subsection{Data Acquisition}

When one of the eight trigger signals (Figure 2.7) made it to the E621 Good Event gate, the data-acquisition process for an event was initiated. Gating signals were sent out to the apparatus electronics and to inhibit further triggers until the event readout was complete. A interrupt signal was sent to control electronics connected to the PDP-11 computer. This "A" interrupt signal caused the computer to immediately start executing a CAMAC readout cycle, as controlled by the data acquisition software. Between each spill, a "B" interrupt was generated to readout scalers and perform calibrations

\subsubsection{MWPC Readout}

Every time a wire in an MWPC had a signal above threshold, the amplifier card with the channel for that wire initiated a 940 ns long "one-shot" signal. This signal was differentiated to provide output signals from both its leading and trailing edges. The leading edge signal, termed the "fast OR", was used in forming triggers. The delayed output maintained the MWPC information from the time a particle passed through the chamber until an $150 \mathrm{~ns}$ long "enable" pulse could be received to hold the information for the readout. The long delay was necessitated by the length of our apparatus, the distance from the data-acquisition trailer to the apparatus (100 ft), as well as the delay of the trigger electronics. During the time this "one-shot" signal was active, all subsequent signals on that wire were ignored. The fraction of the time that a detector element or trigger was unable to respond to new inputs was referred to as the "deadtime". This wire dead-time was a possible source of inefficiency in the chambers. For instance, if the signal rate on a wire was $100,000 / \mathrm{s}(100 \mathrm{kHz})$, then there would be $\sim 10 \%$ inefficiency on the wire due to the dead-time from the one-shot. For this reason, the proton beam intensity was limited so that the overall signal rate for any chamber plane was $<500 \mathrm{kHz}$. Since the rate was spread over several wires, this meant that the wire dead-time inefficiency was kept small. 
The chamber readout electronics[17] were quite reliable. The readout itself was fast as it performed a "sparse readout" of only the amplifier cards with latched signals. There were typically 12-15 cards with signals in an event, The chamber readout's CAMAC interface read out one latched amplifier card every CAMAC cycle $(\sim 2 \mu \mathrm{sec})$. The output format was a unique 16-bit address word for each latched card which contained information on which of the 4 wires on the card were hit.

\subsubsection{ADC and TDC Readout}

ADCs were used to digitize the magnitude of the lead glass block outputs. A 200 ns wide gate was sent to the ADCs (LeCroy 2280's), which digitized the delayed output signals in an integrated charge mode with 12-bit resolution. The ADC controller subtracted the pedestal from each channel. LeCroy 4291 TDCs digitized the time between the receipt of a discriminated lead glass signal and the "Common Stop" signal from the good event gate. They had a 10-bit output, with 1 ns per bit. The high level TDC inputs from an event typically came 150 ns before the Common Stop, while the low level TDC inputs were about $50 \mathrm{~ns}$ before the Common Stop.

\subsubsection{Latches and Scalers}

Delayed signals from all of the counters and triggers were sent to latches, which only recorded those signals in coincidence with a gate sent for each good event. These latches were read out every event. These latch words were used to identify the type of trigger and to check the efficiencies of the counters and triggers.

The outputs of various chamber Fast ORs, counters, and triggers were also sent to scalers, which counted the total number of inputs. Some of these scalers were gated to be active during the entire spill, while other were gated to only be active when the triggers were not being held off. The ratio of these two types of scalers measured the dead-time percentage of the experiment. The scalers were read out and cleared once 
per spill.

\subsubsection{Recording the Event Data}

All data was read out through the CAMAC backplane of the crates. A crate controller in each crate passed its data up a multiwire cable to a Jorway Branch Bus PDP11/CAMAC Interface. A typical event record had about 5016 bit words in it. These event records were combined into 1920 -word (16 bits/word) buffers in computer memory before being written onto a 6250-bpi 9-track magnetic tape. The tape drive buffering was faster than the CAMAC readout driver, so spooling events to disc was not needed. Ignoring trigger dead-time considerations, the read-out could write up to $1000 \mathrm{events} / \mathrm{s}$.

Typically 2400 events were recorded per spill, filling a tape in about two and a half hours. During the digitization and read-out of the apparatus signals no other triggers could be accepted. This read-out dead-time resulted in a triggering inefficiency which was about $15 \%$.

\subsubsection{Between-Spill Readout}

Between every spill, a " $B$ " interrupt event was recorded. For these interrupts, the computer read in the ADC pedestals, and then sent them back to the ADC processor. These pedestals would then be subtracted by the ADC processor from the ADC channels for all events in the subsequent spill. This allowed the ADC's to be read out only for those channels with non-zero signals, which reduced the length of the event record. A command was also sent to the Digital-to-Analog Converter (DAC) on a voltage supply card connected to the ADC's. This card provided the reference voltage level which set the scale for the ADC digitization. This command reset this voltage to the established level to maintain the ADC calibration. A timing signal was sent to the TDC's to allow them to "Zero-Trim" each channel to maintain their calibration. The scalers, which recorded the rates of various triggers and counters, were read out and cleared. 


\begin{tabular}{|c|c|c|c|}
\hline Run & Targets & M1 Polarity & M2,3 Polarity \\
\hline 1 & UE/DW & Normal & Normal \\
2 & UE/DW & Normal & Reversed \\
3 & UE/DW & Reversed & Reversed \\
4 & UE/DW & Reversed & Normal \\
5 & UW/DE & Reversed & Normal \\
6 & UW/DE & Reversed & Reversed \\
7 & UW/DE & Normal & Reversed \\
8 & UW/DE & Normal & Normal \\
\hline
\end{tabular}

Table 2.3: Targets and magnet polarities during an 8 run cycle

\subsection{Experiment Operation}

The runs, one per tape, were organized in cycles of 8 . As shown in Table 2.3, for most cycles the first four runs were taken with US-East/DS-West targetting, and the second four with US-West/DS-East. The polarity of collimator magnet M1 was reversed every four runs, while the polarity of the M2 and M3 spectrometer magnets was reversed every two runs. This was all to average over any spatial asymmetries in the apparatus. Towards the end of the experiment, the vacuum seal on the collimator channel became sensitive to the M1 polarity reversals. To minimize the rupturing of this seal, the reversing of the M1 polarity was done much less frequently.

As has been discussed, the F.C5,$\Lambda$ and $\Xi^{0}$ triggers were passed through prescalers during most of the experiment. On a typical tape of 400,000 events, there were only 20-35,000 events from each of these three triggers. Towards the end of the experiment, ten runs were taken expressly for $\Xi^{0}$ data. The $\Xi^{0}$ trigger prescaler was set to 0 , and the $\Lambda$ trigger prescaler set to $2^{6}$. These runs yielded nearly a third of the $\Xi^{0}$ data set.

All the beamline elements were monitored and controlled to ensure that the targetting was maintained and any drifts were corrected. The $K_{\pi 3}$ yield from each target was analyzed as a function of the trigger rates and beam intensity. The results of this analysis were used in beamline controls software which adjusted the septum position to maintain the correct beam fractions on each target. 
To monitor the performance of the chambers, radioactive sources of $\mathrm{Fe}^{55}$ were mounted in the corner of each chamber outside of its active area. The wires under the $5.9 \mathrm{KeV}$ x-ray source were connected to an emitter-follower amplifier and routed to a panel in the trailers, where they could be observed on an oscilloscope. When operating properly, the signal was about $5 \mathrm{mV}$ in height. These could be used to adjust the high voltage to maintain chamber efficiency, which could vary due to fluctuation in the gas mixture and pressure.

\subsubsection{Chamber Alignment}

The chambers were optically surveyed and levelled when they were originally installed. The initial centerline of the chambers was established along the center wire of each plane. A more accurate alignment was done during a special "straight-through" run. A low intensity proton ( $1^{6}$ protons/spill) beam was guided with the beamline elements to the west hole of the collimator. For this run, both the sweeper and analyzing magnets had been turned off. This ensured that the proton beam described a straight line through the apparatus. The center of the beam profile in each MWPC plane was defined as the origin of the coordinate system at that plane. These values were refined in the actual reconstruction to correct for shifts in the locations of the wire planes due to removal of the planes during the experiment to fix broken wires.

\subsubsection{Lead Glass Calibration Runs}

To calibrate the lead glass calorimeter, and balance the gains of individual blocks so the response would be as uniform as possible, special " $e^{+} e^{-}$" calibration runs were made. The beamline was tuned to provide a single proton beam which would interact in the US target in the East position. Using this target greatly reduced the number of $\Lambda$ and $K_{S}^{0}$ decays in the spectrometer, as most of these particles would decay upstream of the decay volume. A small sheet of lead was put into the vacuum pipe just ahead of 
the V1 counter. Photons interacting in it would typically produce an positron-electron pair $\left(e^{+} e^{-}\right)$. As photons and electrons have a similar electromagnetic shower, the array could be calibrated by relating the pulse heights in the lead glass ADC's to the momentum of the electrons measured in the charged-particle spectrometer. To steer electrons into most blocks of the array, for these runs we also used the kicker magnet MV located just after V1. It had a horizontal field so that it split the $e^{+} e^{-}$pair vertically. Varying the MV field strength and polarity as well as the settings of the analyzing magnets which bent horizontally was used to take calibration runs with different blocks of the array begin illuminated by the $e^{+} e^{-}$tracks. To illuminate the central blocks, data was taken with the array displaced $28 \mathrm{in}(71.1 \mathrm{~cm})$ to the right.

The trigger was suitably changed to trigger on these decays. This trigger was

$$
V 1 \cdot D K \cdot(A \cdot B) \cdot\left(\Pi_{O R}+V_{O R}\right) \cdot P b G_{O R}
$$

where $A, B$ denoted signals in both sides of the $A, B$ hodoscopes, $\Pi_{O R}$ the OR of all the $\pi$-killer counters, $V_{O R}$ the aforementioned OR of the VT and VB counters, and $P b G_{O R}$ an OR of all the blocks in the $\gamma$ trigger. Several million reconstructable $e^{+} e^{-}$events were recorded with which to study the lead glass array response.

\subsection{Detector Performance}

\subsubsection{Chamber Efficiency}

The reconstructed neutral decay events were analyzed to measure the inefficiency of the chambers on a run-by-run basis. The averages for each of the twelve planes over the entire experiment for a sample of $\Lambda$ decays are listed in Table 2.4. While these apparent inefficiencies were not necessarily the actual single-track inefficiencies, they were proportional to them and provided a check that the chambers were operating properly. The $x$-plane inefficiencies were underestimated because the $x$-plane fits had only two to three planes in them, and hits from two planes were required in the fit. 


\begin{tabular}{|c|c|c|c|c|c|c|}
\hline Plane & \multicolumn{6}{|c|}{ Chamber No. } \\
\cline { 2 - 7 } & 1 & 2 & 3 & 4 & 5 & 6 \\
\hline Y(or V) & 4.91 & 4.64 & 4.85 & 6.09 & 4.95 & 8.45 \\
\hline $\mathrm{X}$ (or U) & 1.47 & 2.92 & 2.78 & 1.24 & 4.88 & 4.40 \\
\hline
\end{tabular}

Table 2.4: Inefficiency (\%) of MWPC planes measured from reconstructed events

The $y$-fits, which typically had four to five planes in them, were less sensitive to this selection bias and gave a truer picture of the performance of a chamber.

\subsubsection{Trigger Counter Efficiencies}

The efficiencies of the trigger counters were determined from the latch information for reconstructed F.C5 trigger events. The F.C5 trigger data was used because it did not have these counters in its logic.

In general, the trigger counters had efficiencies which were $>98 \%$. A few of the A and $B$ hodoscope elements had significant 10-20\% inefficiencies during some periods of the experiment. The events from the $\Lambda$ trigger were then compared against distributions of Monte Carlo (See Chapter 4 events which were generated with the counter efficiencies assumed constant. This was done to verify that the latch information was reflecting a true inefficiency in the counter and not just a faulty latch. The observed efficiencies were then used in generating the final MC samples.

\subsubsection{Lead Glass Energy and Position Resolution}

The energy and position resolution of the lead glass array was determined from the $e^{+} e^{-}$calibration run (Section 2.8.2) events. The energy resolution was determined by plotting the reconstructed cluster energies $(E)$ as a function of the momentum $(P)$ of the electron or positron, and finding the standard deviation of the Gaussian which best fit the distribution for each momentum bin. With the lead pre-converter in front of the array, the energy resolution was $\sigma(E) / E=0.22 / \sqrt{E}$, where $E$ is the energy in $\mathrm{GeV}$, 
$\mathrm{Pb}$ Glass Energy Resolution

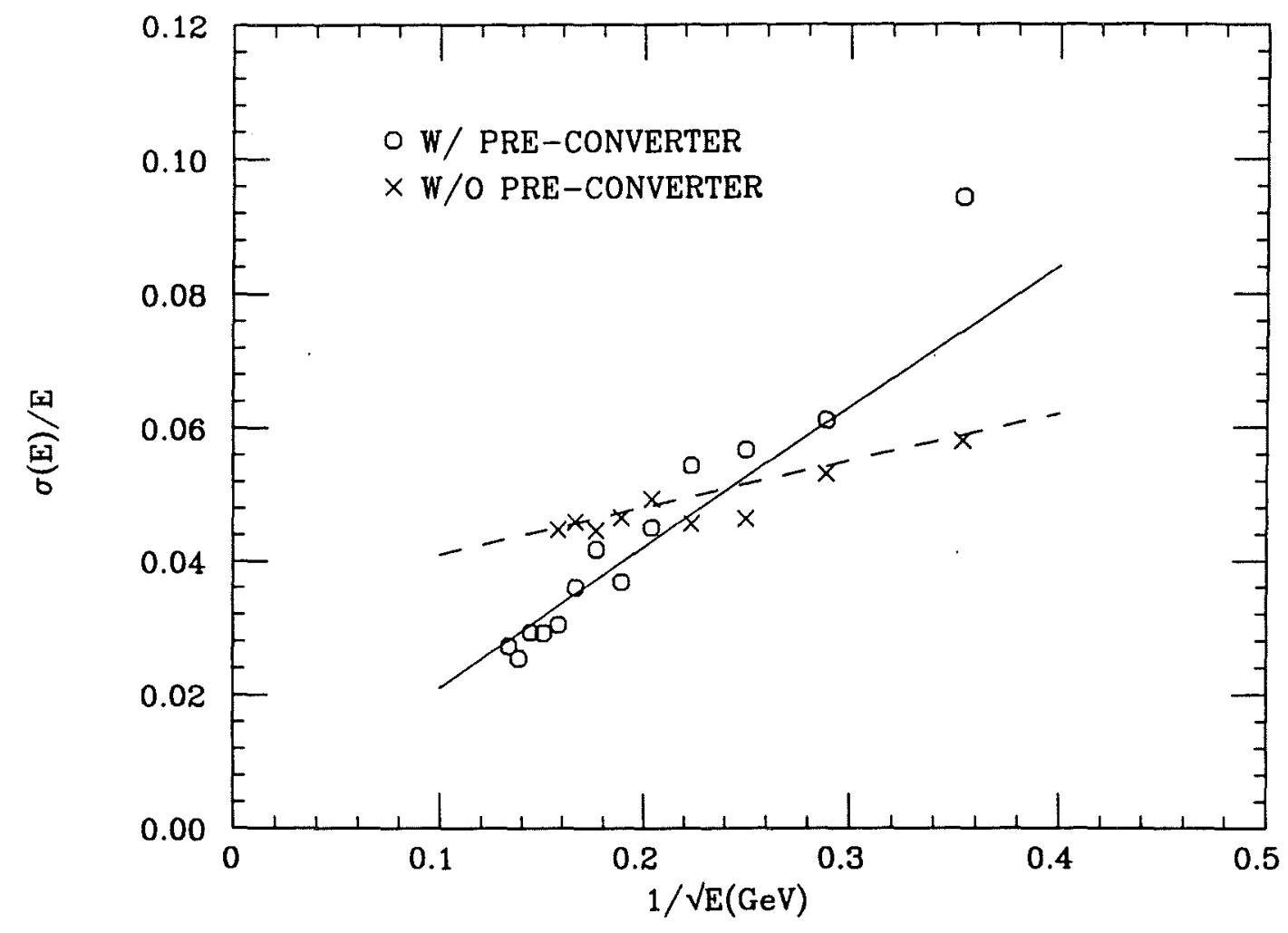

Figure 2.13: Energy resolution of the lead glass array both with and without the pre-converter. The lines indicate the fits described in the text.

while with the pre-converter removed, the resolution was $\sigma(E) / E=0.032+0.07 / \sqrt{E}$ (Figure 2.13). The resolutions are averaged over the entire array for clusters more than half a block $(5.58 \mathrm{~cm})$ from the hole and outer edge of the array. Clusters near the hole had a poorer energy resolution. To correct for the energy lost down the hole in such clusters, a separate set of gain constants was used for the six blocks next to the hole when the cluster center was within one half-block of the hole. These constants were typically $10 \%$ larger than the normal constants for these blocks.

The linearity of the response of the lead glass array as a function of energy was determined by plotting the average $E / P$ ratio. The response (Figure 2.14) of the array 
$\mathrm{Pb}$ Glass Linearity

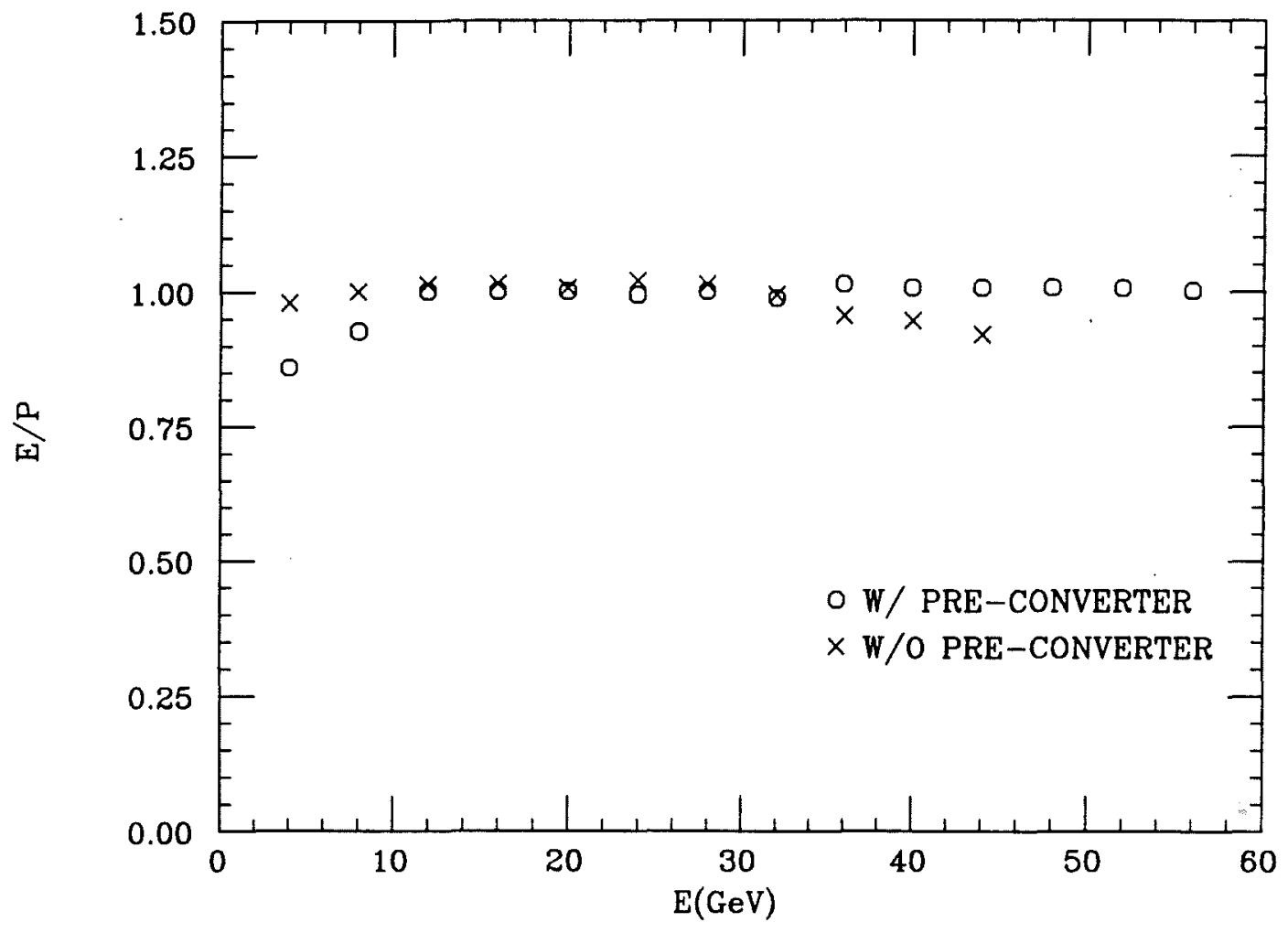

Figure 2.14: Linearity of lead glass array response(E) as a function of track momentum (P) for $e^{+} e^{-}$events 
for most of the experiment, when the pre-converter was in place, was flat above $10 \mathrm{GeV}$, but fell off with decreasing energy for energies less than $10 \mathrm{GeV}$. The response with the lead pre-converter removed was flat for low energy, but fell off steadily above $20 \mathrm{GeV}$. In both cases, rather than correct for this in the analysis, the non-linearity measured from the $e^{+} e^{-}$data was put into the MC simulation.

The first approximation $\left(X_{C L U S T E R}, Y_{C L U S T E R}\right)$ to the center of the reconstructed shower was calculated using first moment equations, one for the $\mathrm{x}$ coordinate

$$
X_{C L U S T E R}=\frac{\sum_{i=1}^{n} X_{i} E_{i}}{\sum_{i=1}^{n} E_{i}}
$$

and one for the y coordinate. The summation was over all blocks in the cluster. $E_{i}$ was the energy and $X_{i}$ the $\mathrm{x}$ position of the center of block $i$ of the cluster. The accuracy of this approximation was analyzed by using the $e^{+} e^{-}$data. These events were first binned by the value of $\left|X_{C L U S T E R}-X_{B L O C K}\right|$, where $X_{B L O C K}$ was the $\mathrm{x}$ position of the center of the block within whose edges $X_{C L U S T E R}$ lay. This procedure served to combine all the data from the different blocks in the array into one plot. Then for each such bin, the average of the $x$ positions of the reconstructed $e^{+}$or $e^{-}$from each event projected to the face of the lead glass array was found. Again, these position were taken as the offset from the center of the block. This average, termed $\overline{X_{T R A C K}}$, was plotted as a function of $X_{C L U S T E R}$, which is shown in Figure 2.15. These calculations were repeated for the $y$ coordinate. If the first moment calculation had accurately determined the actual center of the clusters, then for each bin $\overline{X_{T R A C K}}$ should have equaled $\left|X_{C L U S T E R}-X_{B L O C K}\right|$. The plots in Figure 2.15 clearly show that this was not the case. The only agreement was at the very center of the main block, and on the edge between blocks, where typically equal amounts of the energy of the cluster went into the two adjoining blocks. This systematic error in the first moment calculation was due to the non-uniformity of the energy distribution within photon clusters, which was in fact highly centralized. Because the first moment calculation did not weight cluster contributions from outside the main block heavily enough, it gave a position which, on 
Avg. $X_{\text {TRACK }}$ vs. $X_{\text {CLUSTER }}$

Avg. $Y_{\text {TRACK }}$ vS. $Y_{\text {CLUSTER }}$
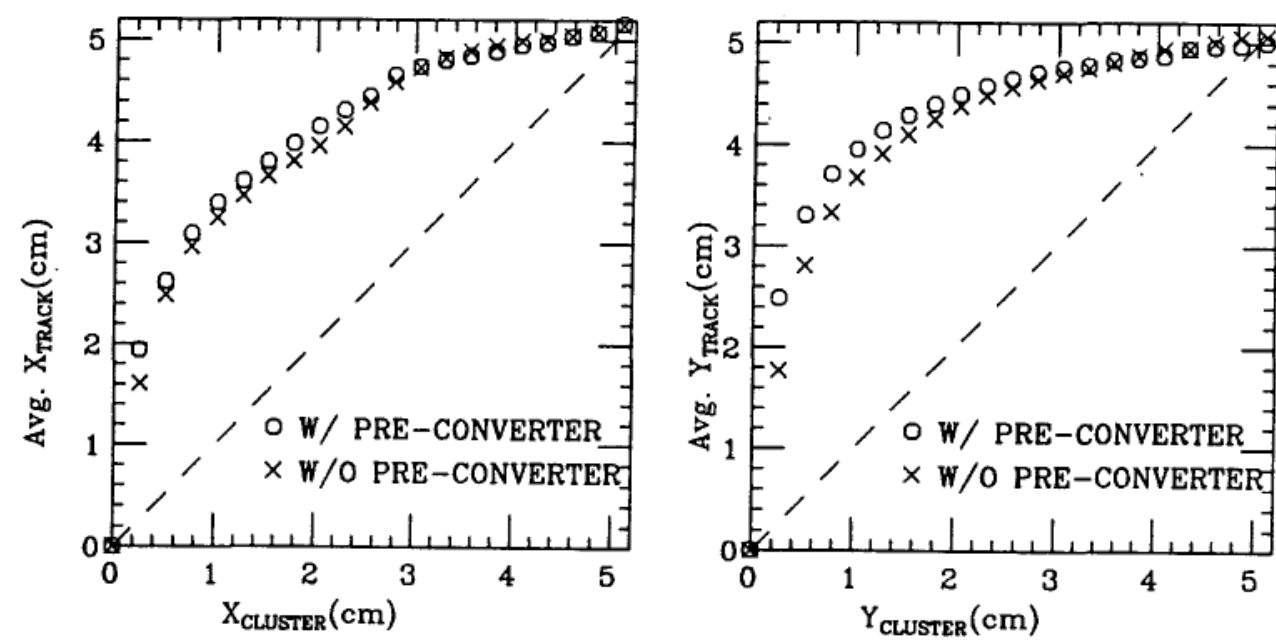

Figure 2.15: Average positions $\overline{X_{T R A C K}}$ and $\overline{Y_{T R A C K}}$ of the $e^{+}$and $e^{-}$tracks as a function of the calculated cluster center coordinates, $X_{C L U S T E R}$ and $Y_{C L U S T E R}$ respectively. These quantities are all taken as offsets from the center of the main block of the cluster. Please note that only the absolute values are used. 0 is the block center, and $5.08 \mathrm{~cm}$ the nominal edge of the block. Note the large deviation of the plots from equality of the $\overline{T R A C K}$ and CLUSTER quantities, which is represented by the dashed line. 
average, was too close to the center of the main block when compared to the projected track. To correct the calculated center of a reconstructed photon cluster in the $\Xi^{0}$ data for this systematic error, first the offset of $X_{C L U S T E R}$ from the center of the main block was found. Next, the corresponding value of $\overline{X_{T R A C K}}$ was interpolated from the data of Figure 2.15, and then the absolute value of the center restored by adding back the $\mathbf{x}$ position of the main block center. These corrected shower centers, for showers not near the edges of the array, had position resolutions of $\sigma_{x}, \sigma_{y}=1.1 \mathrm{~cm}$ with the lead pre-converter on, and $\sigma_{x}, \sigma_{y}=1.2 \mathrm{~cm}$ with the lead pre-converter off, as determined from the $e^{+} e^{-}$data.

Originally, it had been intended to take only a few sets of calibration runs, and to use the $\pi^{0}$ mass as a constraint in the $\Xi^{0}$ and $K_{\pi 3}$ event reconstructions to check the run-by-run drifts in calibration. It turned out that the neutral beam intensity was high enough to cause significant radiation damage in the central blocks of the array, and to darken them over time, reducing their response. This damage caused a steady decrease in the $\pi^{0}$ mass in reconstructed events, if it was assumed that the gain constants were constant over time. To combat this degradation of the array, periodically a portion of the array was unstacked and blocks in the center were swapped with spare clear ones. The photomultiplier tubes were kept in the same places in the array. The intense UV light of two mercury vapor lamps to was used to clear up the darkened blocks between restackings. After each re-stack, a new set of $e^{+} e^{-}$calibration runs was taken to rebalance the gains and to adjust the timing of block signals. 


\section{Chapter 3}

\section{Event Reconstruction}

\subsection{Introduction}

The events recorded on the data tapes during the experiment were analyzed to identify neutral particle decays. If evidence for a decay was found in an event, the analysis program attempted to reconstruct the decay vertices, particle momenta and positions from the MWPC signals. These quantities were used to identify $\Lambda$ decays. Those events with an identified $\Lambda$ decay were further analyzed for evidence of photon showers in the lead glass array. Events from the $\Xi^{0}$ trigger which had a $\Lambda$ decay and two reconstructed photon showers were then analyzed to see if they were consistent with being a $\Xi^{0}$ decay. If so, a fit was made to reconstruct the $\Xi^{0}$ decay vertex and momenta.

\section{2 $\Lambda$ reconstruction}

The reconstruction of the $\Lambda \rightarrow p \pi^{-}$decay in an event was performed by fitting the "hit" wires recorded by the six MWPC's. The first step was to attempt to fit these hits to a "neutral vee" topology and to determine the decay vertex and charged particle tracks. If this purely geometric fit was successful, then the momentum of each of the charged particles was calculated using the angles their tracks were bent through by the 


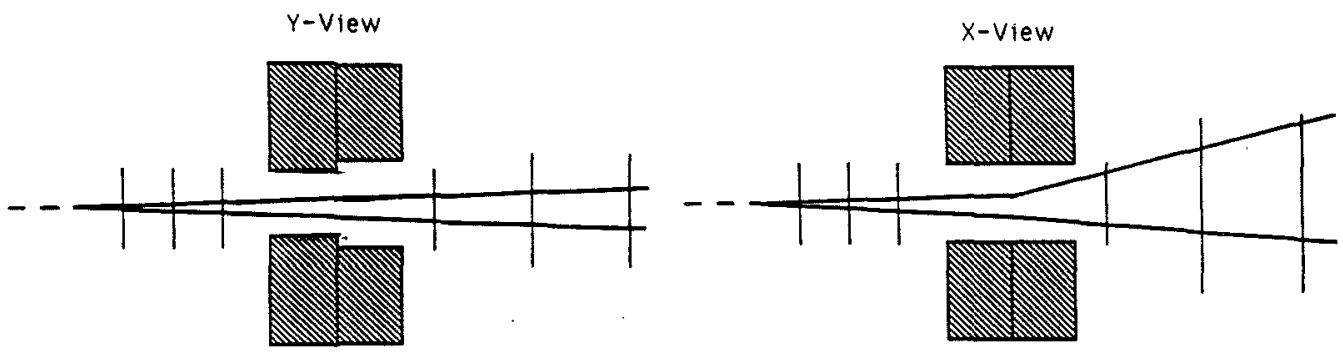

Figure 3.1: Neutral vee fit topology

field of the analyzing magnets. These momenta allowed the invariant mass of the decay to be calculated under different hypotheses for the identities of the charged particles. These invariant mass calculations were used to identify the $\Lambda$ decays. Once the decay particle was identified, a more complete kinematic fit, using the known particle masses as constraints, was performed to enhance the decay vertex resolution.

\subsubsection{Geometric Fit}

The charged track topology required in the fit was a "neutral vee", as shown in Figure 3.1. In the $y$ view, it required two straight tracks which diverged from a point upstream of the first chamber. In the $\mathrm{x}$ view, two tracks were required upstream of the magnets which diverged from this decay point. The upstream tracks had to match up with two tracks in the chambers downstream of the magnets, meeting near the effective bend plane of the magnets. The fitting program first searched for divergent straight tracks in the $y$ view, starting at the downstream chambers and working upstream. In $x$, the program began by fitting the pre- and post-magnet hits separately to straight lines, then attempted to match them up at the effective bend plane. The fitted tracks were required to bend in opposite directions at the magnet bend plane, as well as to have a vertex upstream of $\mathrm{C} 1$ with a z-coordinate consistent with that in the $\mathrm{y}$ view. Lastly, 


\begin{tabular}{|c|r|r|r|r|r|r|}
\hline \multirow{2}{*}{$\begin{array}{c}\text { Reconstruction } \\
\text { Result }\end{array}$} & \multicolumn{3}{|c|}{ F.C5 Trigger } & \multicolumn{3}{c|}{$\Lambda$ Trigger } \\
\cline { 2 - 7 } & \multicolumn{2}{|c|}{ Data } & MC & \multicolumn{2}{c|}{ Data } & MC \\
\cline { 2 - 7 } & Events & $(\%)$ & $(\%)$ & Events & $(\%)$ & $(\%)$ \\
\hline Triggers & $\mathbf{3 , 4 5 7 , 2 0 0}$ & - & - & $3,181,400$ & - & - \\
Neutral Vee & 864,300 & 25.0 & 74.8 & $2,083,800$ & 65.5 & 81.8 \\
$\Lambda$ & 622,300 & 18.0 & 74.5 & $1,177,100$ & 37.0 & 81.5 \\
$\bar{\Lambda}$ & 25,180 & 1.0 & - & 56,530 & 1.5 & - \\
\hline
\end{tabular}

Table 3.1: Yields of reconstructed $\Lambda$ events

\begin{tabular}{|c|r|r|r|}
\hline \multirow{2}{*}{$\begin{array}{c}\text { Reconstruction } \\
\text { Result }\end{array}$} & \multicolumn{3}{|c|}{$\Xi^{0}$ Trigger } \\
\cline { 2 - 4 } & \multicolumn{2}{|c|}{ Data } & MC \\
\cline { 2 - 4 } & Events & $(\%)$ & $(\%)$ \\
\hline Triggers & $7,074,800$ & - & - \\
Neutral Vee & $3,183,200$ & 45.0 & 92.0 \\
$\Lambda$ & $1,697,700$ & 24.0 & 91.8 \\
$\bar{\Lambda}$ & 95,530 & 1.4 & - \\
\hline$\geq 2 \gamma^{\prime} s$ & 499,030 & 7.1 & \\
\hline
\end{tabular}

Table 3.2: Yields of reconstructed $\Xi^{0}$ events

the $u$ and $v$ plane hits in $\mathrm{C} 2$ were used to match up the $\mathrm{y}$ tracks with corresponding $\mathrm{x}$ tracks. The geometric fit did not require a hit for every track in every chamber, and so was tolerant of small chamber inefficiencies.

The "residual" for a track from a plane was the distance between the fitted track position and the actual position of the hit. These residuals were used to form a $\chi^{2}$. The average Gaussian $\sigma$ for these residuals was $0.04 \mathrm{~cm}$ The track vectors and decay vertex were adjusted until the $\chi^{2}$ was minimized to determine the best fit. The yields of reconstructed events is shown for the F.C5 and $\Lambda$ triggers in Table 3.1, for both data and Monte Carlo $\Lambda$ samples. Table 3.2 shows the breakdown for the $\Xi^{0}$ trigger data and MC samples.

Events were rejected on the basis of several criteria. The fraction of real events with more than two tracks in the $y$ view was due to interactions of neutral beam particles with the material in the apparatus. The events lost due to an insufficient number of hits in the chambers were due both to chamber inefficiencies and to $\pi^{-}$'s which did not pass 
through all the chambers. The $\Lambda$ and $\Xi^{0}$ triggers lost fewer events from this than the F.C5 events because the $\Lambda$ trigger required a $\pi^{-}$track downstream of the magnets. The small opening angles and high momentum of many $\Lambda$ decays caused the fit to fail due to the inability to determine conclusively which of the two $y$ tracks went with which of the $x$ tracks. Events which had no apparent separation of the tracks upstream of the magnets were labelled " $\gamma \rightarrow e^{+} e^{-"}$ decays. They were due both to actual $\gamma \rightarrow e^{+} e^{-}$events in the real data, and $\Lambda$ decays with insufficient opening angles. The $\chi^{2}$ per degree of freedom (d.o.f.) of the geometric fit for $\Lambda$ events is shown in Figure 3.2.1. Only events which had a $\chi^{2} /$ d.o.f. less than 3.0 were used. Note that in this figure, the distribution for the real events is indicated by the circles with accompanying error bars indicating the statistical uncertainty. The corresponding distribution for the Monte Carlo events (Chapter 4), normalized to the real event distribution, is indicated by the histogram. This format is used throughout this and the following chapter, except where only real event distributions are shown. In those cases, only a histogram showing the real events is shown.

\subsubsection{Chamber centers}

The chambers were initially aligned by using the set of chamber centers found with the alignment run described in Section 2.8.1. A closer examination of the run-by run behavior was made by studying the averages of the residuals from the fitted events. The averages had offsets from zero on the order of $0.1 \mathrm{~mm}$. Two sources for these offsets were determined. A few times during the experiment a chamber had to be removed to fix a wire which had broken inside it. Only the chamber wire planes were removed to make the repairs, leaving the chamber structure in place. The planes were expected to have returned to the same positions when re-installed. However, a few systematic shifts on the order of $0.2 \mathrm{~mm}$ were seen due to these repairs. For the final analysis, six different sets of chamber center values were used, one each for every time a chamber 


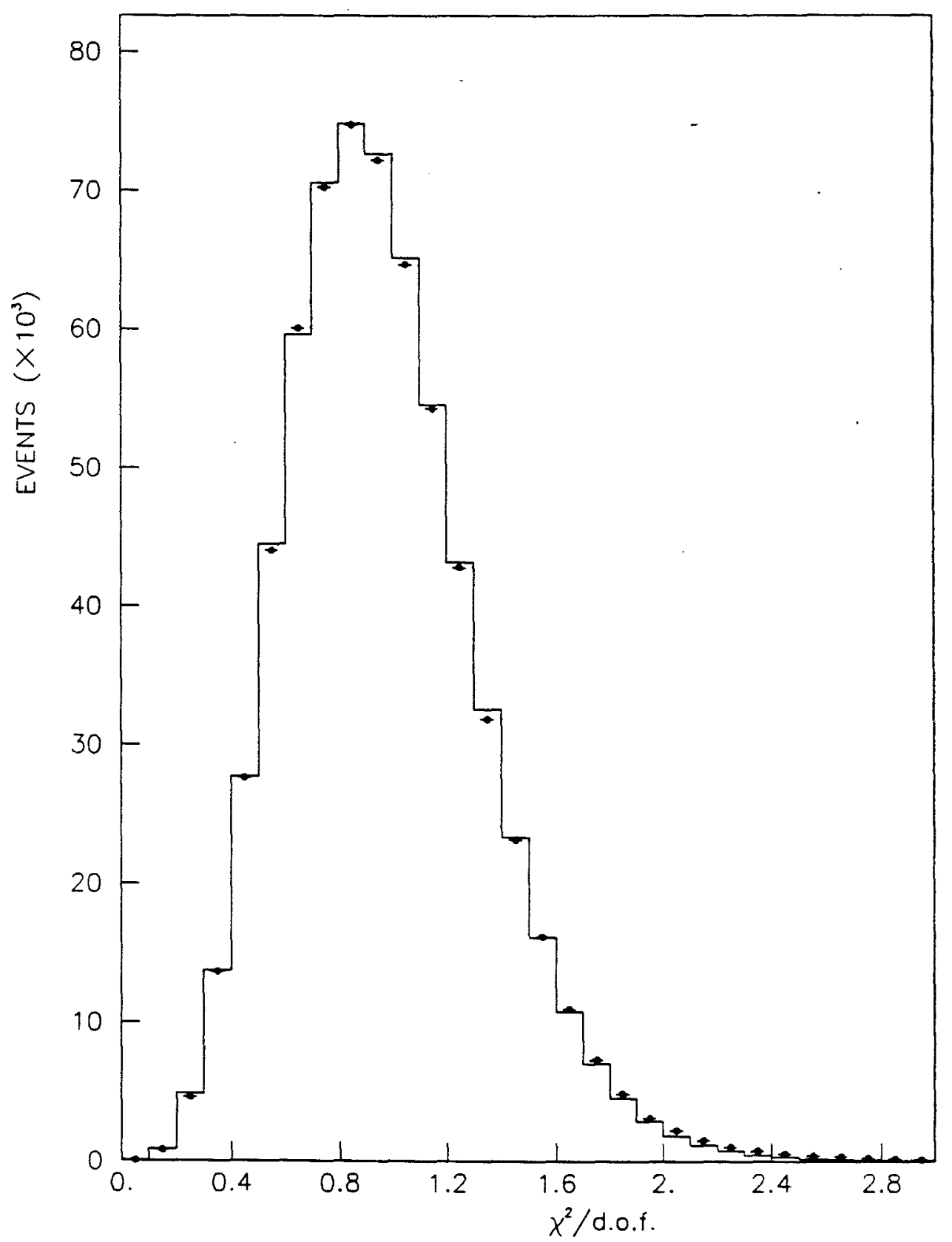

Figure 3.2: $\chi^{2}$ per d.o.f. of the geometric fit for $\Lambda$ events. Only events with a $\chi^{2} /$ d.o.f. $<3.0$ were used. Note that the real $\Lambda$ events are plotted as the "dots" with statistical error bars, while the "histogram" shows the cooresponding MC event sample. This format continues for all the following such plots. 
was removed for repair. The rest of the run-to-run variations, which often were as large as the above systematic effects, were assumed to be due to random fluctuations in the height of the PC pit floor and differential expansions due to temperature fluctuations. It was decided not to correct for these fluctuations in the analysis, as they made no systematic effect. We instead chose to model this behavior in the MC by varying the assumed centers run-by-run, based on the observed offsets of the residuals in the $\Lambda$ data.

The events were studied to determine whether there were significant relative rotations of the chambers about the z-axis. This would be revealed by the y-residual having a dependency on $\mathrm{x}$, and a corresponding $\mathrm{x}$-residual dependence on the $\mathrm{y}$ position in the chamber. All the rotations were found to be $<1$ mrad., and making a correction for them in the analysis had no effect on the $\chi^{2}$ of the fit.

\subsubsection{Analysis Magnet Fields}

The magnetic field in the two analysis magnets was oriented in the $y$ direction $\left(B_{Y}\right)$. This field caused the transverse momentum component $P_{x z}$ of the charged particles to be rotated about the $\mathrm{y}$ axis as they passed through the magnets. This momentum rotation was reflected in the relative angle $\phi$ between the $\mathrm{x}$-view particle tracks in the chambers upstream and downstream of the magnets. This angle is related to the transverse momentum kick $P_{T}$ of the magnetic field.

$$
\sin \phi=P_{T} / P_{x z}
$$

For a uniform field of magnitude $B$ in kiloGauss and length $L$ in centimeters, the $P_{T}$ is defined as

$$
P_{T}=3 \times 10^{-4} B L
$$

in units of $\mathrm{GeV} / \mathrm{c}$.

To fit together the upstream and downstream $x$-view tracks, an effective bend plane 


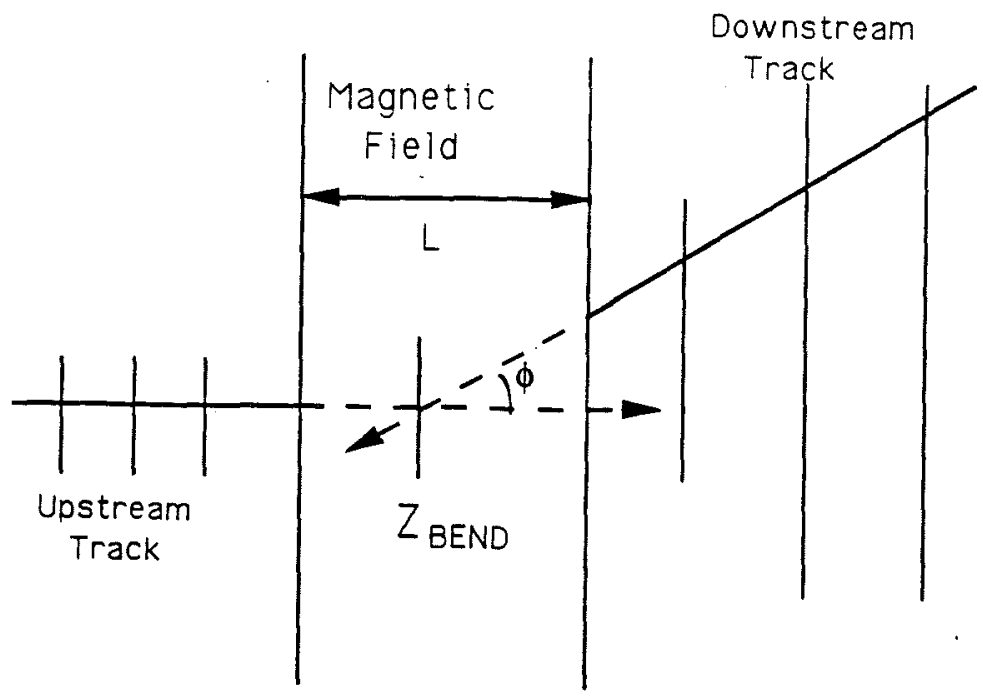

Figure 3.3: Effective bend plane $Z_{B E N D}$

was used(Figure 3.3). For a uniform magnetic field of length $\mathrm{L}$, this $Z_{B E N D}$ plane is at the mid-point $(\mathrm{L} / 2)$ of the field, with small corrections due to the bend angle $\phi$ and the entrance angle $\alpha$.

In this experiment, there were actually had two magnets of the same length but with different magnetic field strengths with a gap between them. The expression for the position of this effective bend plane relative to the beginning of the first magnet, expanded to lowest order in $\phi$ and $\alpha$, was a function of the field lengths $\ell$ and $\ell^{\prime}$, the gap $s$, and the ratio $\lambda$ of the the first magnet's $P_{T}$ to that of both magnets together.

$$
\begin{aligned}
Z_{B E N D}= & \frac{1}{2}\left[(\ell+s)(2-\lambda)+\left(\ell^{\prime}+s\right)(1-\lambda)-s\right] \\
& +\frac{1}{2}\left[(\ell+s) \lambda(3-2 \lambda)+\left(\ell^{\prime}+s\right)(1-\lambda)(1+2 \lambda)\right] \alpha \phi \\
& +\frac{1}{2}\left[(\ell+s) \lambda\left(2-\lambda^{2}\right)+\left(\ell^{\prime}+s\right)(1-\lambda)(1+\lambda)^{2}\right] \phi^{2}
\end{aligned}
$$

The nominal value for $Z_{B E N D}$, equivalent to the first line of Equation 3.4, was in the limit that $\phi$ and $\alpha=0$. As the two magnets were run at the same current but had different coil separations, $10 \mathrm{in}(25.4 \mathrm{~cm})$ for the first and $12 \mathrm{in}(30.48 \mathrm{~cm})$ for the second, 


\begin{tabular}{|l|c|c|}
\hline Parameter & Units & Magnitude \\
\hline Magnet 1 Length & $\mathrm{cm}$ & 184.7 \\
Magnet 2 Length & $\mathrm{cm}$ & 188.8 \\
Gap & $\mathrm{cm}$ & 44.5 \\
$\lambda=P_{T}(1) / P_{T}$ & & 0.578 \\
$Z_{B E N D}$ & $\mathrm{~cm}$ & 189.1 \\
$P_{T}$ & $\mathrm{GeV} / \mathrm{c}$ & 1.554 \\
\hline
\end{tabular}

Table 3.3: Analysis magnet field parameters used in the reconstruction. Note that the $Z_{B E N D}$ value above is given as offset from the beginning of the first magnet's field. In terms of the experiment coordinates, it was $3333.0 \mathrm{~cm}$.

the ratio $\lambda$ was expected to have been $>0.5$. In a previous experiment[20] with the same magnets, a value of 0.545 was used for $\lambda$. That experiment also used values of $184.7 \mathrm{~cm}$ and $188.8 \mathrm{~cm}$ for the effective field lengths of the two magnets. In principle, the nominal $Z_{B E N D}$ could have been calculated from these field lengths, the length of the field-free gap between the magnets, and this field ratio of 0.545 . In practice, the nominal value of $Z_{B E N D}$ was adjusted to optimize the yield of reconstructable events, which also minimized the average $\chi^{2}$ for reconstructed events, which turned out to be $3333.0 \mathrm{~cm}$ from the downstream face of the collimator. The value of $\lambda$ was then adjusted to $\mathbf{0 . 5 7 8}$, so the effective bend plane expression (Equation 3.4 ) yielded this value for $Z_{B E N D}$. The field parameters used in the event reconstruction and the MC are shown in Table 3.3. Using these parameters in the MC event simulation resulted in consistent $\chi^{2}$ and reconstructed $\Lambda$ masses.

The value of total $P_{T}$ used was set to a value which gave the reconstructed $K^{0}$ events the accepted rest mass of $497.7 \mathrm{MeV} / \mathrm{c}^{2} . K^{0}$ decays, rather than $\Lambda$ decays, were used because their reconstructed mass is seven times as sensitive to the magnitude of $P_{T}$. The $K^{0}$ data was checked for several different runs to refine the $P_{T}$ value. It was concluded that there was no significant run-to-run variation needed in $P_{T}$. The value used in the final analysis was $1.5535 \mathrm{GeV} / \mathrm{c}$.

A field map of the analysis magnets was made several months after the run using the Fermilab "Ziptrack" apparatus. The magnets were not in exactly the same relative 
positions, as they had been moved $12 \mathrm{in}(30.48 \mathrm{~cm})$ apart along the z-axis in preparation for the next experiment. This map (Figure 3.4) showed that the dominant $B_{Y}$ magnetic fields were quite uniform inside the magnet and fell to near zero at the aperture of the mirror plates on either end of each magnet. The effective field lengths extracted from this measurement were $189.7 \mathrm{~cm}$ for the first magnet and $191.6 \mathrm{~cm}$ for the second. The maximum $B_{Y}$ fields measured in the center of each magnet were $15.68 \mathrm{kG}$ and $11.39 \mathrm{kG}$ respectively. Using these quantities to calculate the $P_{T}$ of each magnet, a value for $\lambda$ of 0.577 was obtained. This was consistent with the value for $\lambda(0.578)$ derived from the optimization of the reconstruction $\chi^{2}$ mentioned above.

At the ends of the magnets the $B_{Y}$ fields varied rapidly with the $z$ position. This change induced a small z-component to the field $\left(B_{Z}\right)$ at that point. These "fringe" fields were zero at the mid-plane, but rose with increasing $y$ above and below the mid-plane. The $B_{Z}$ fields measured by the Ziptrack at a point $3.0 \mathrm{~cm}$ above the midplane are shown in Figure 3.4 superimposed on the $B_{Y}$ fields. These $B_{Z}$ fields were only significant at the four ends of the $B_{Y}$ fields. These fields coupled to the $\mathrm{x}$ and $\mathrm{y}$ components of a particle's momentum, and thus only had a significant effect when $P_{x y}$ was large. This occurred at the exit of the second magnet, where the $x$-momentum had been boosted to at least $1.5 \mathrm{GeV} / \mathrm{c}$ by the $B_{Y}$ fields. The fringe fields acted to slightly focus the particles in the $y$-view as a function of momentum and the distance above or below the horizontal mid-plane of the magnet gaps. The effect was greatest for the low energy $\pi$ 's. This gave a kink to the y-tracks not accounted for in the reconstruction. Evidence for this deflection of the y-tracks was seen in the data by looking in the tails of the distribution of y-residuals at C5 (Figure 3.5). This small effect was not corrected for in the event reconstruction, but instead the MC simulated the effect of these fringe fields. 


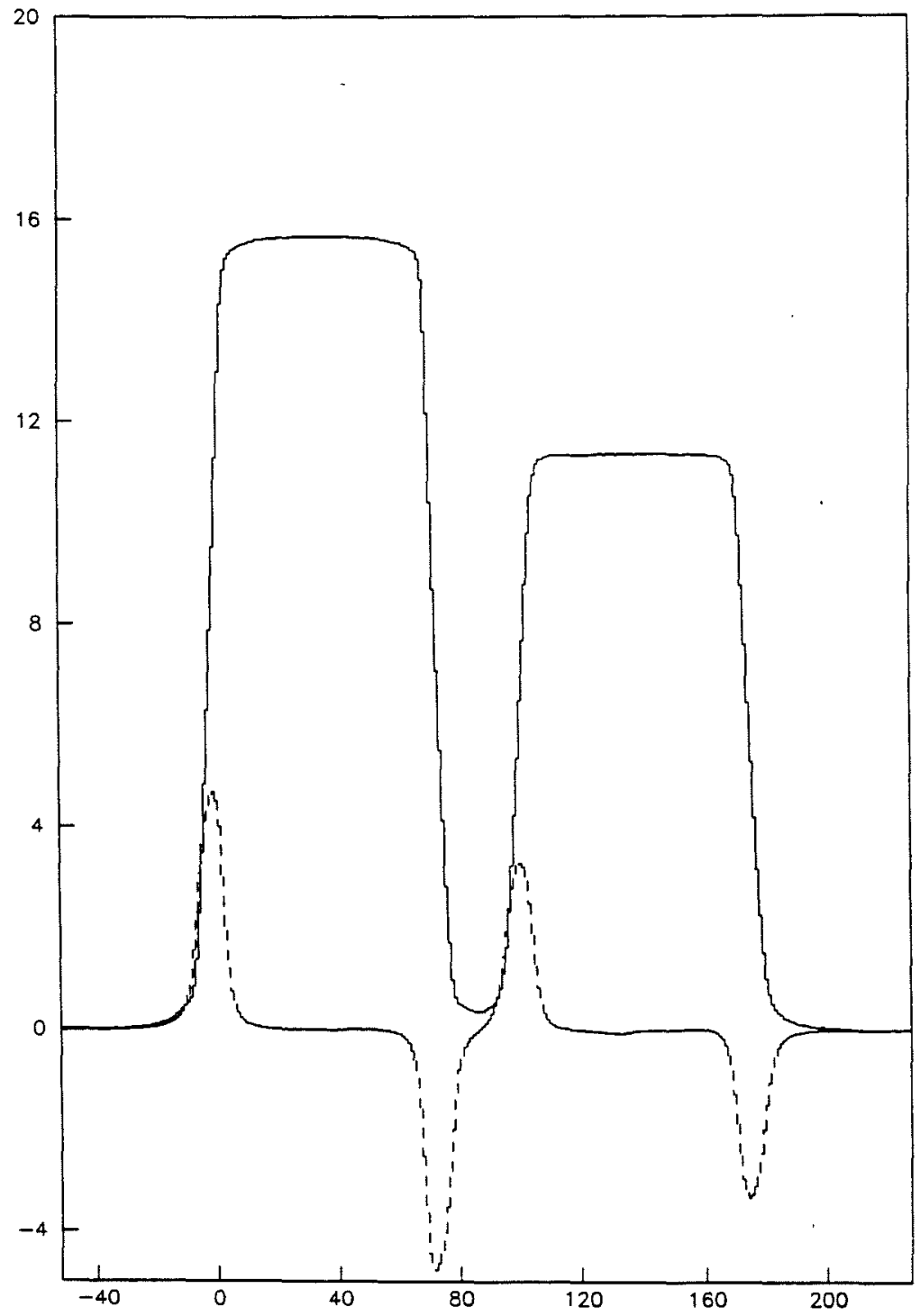

Figure 3.4: Map of $\mathrm{Y}$ and $\mathrm{Z}$ - Axis Magnetic Field Components. The $B_{Z}$ fringe fields indicated were those measured at $3.0 \mathrm{~cm}$ above the midplane of the magnets 


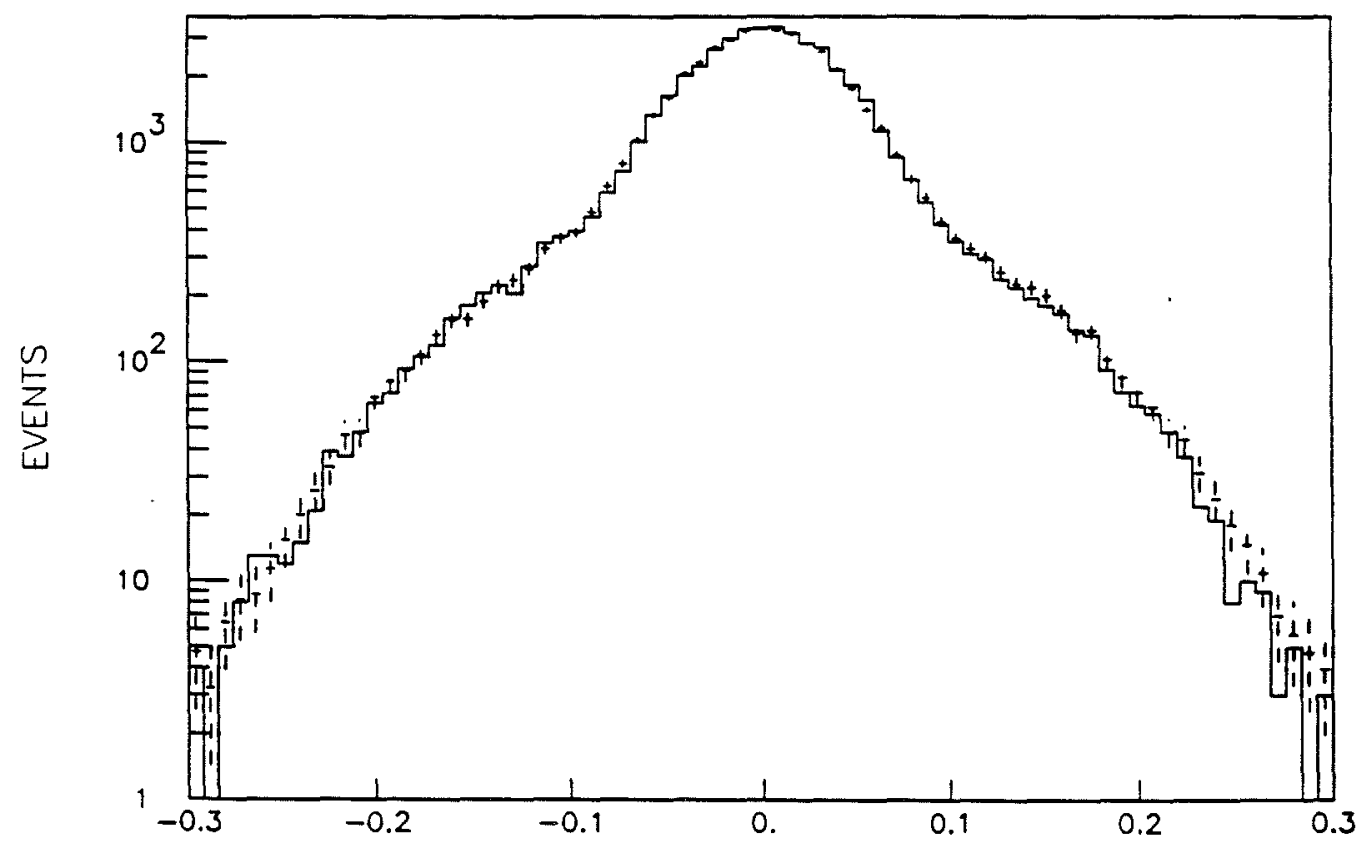

Figure 3.5: Distribution of $Y$ residuals at chamber 5 for $\pi^{-}$tracks. A logarithmic scale is used to show the tails due to the bending from the fringe fields. Note that the real event distribution is plotted as the "crosses" with statistical error bars, while the "histogram" shows the cooresponding MC event sample. 


\subsubsection{Decay Particle Identification}

The identification of the decay particle was done indirectly, by making hypotheses about the identities of the two charged particles and comparing the reconstructed rest mass to that of candidate neutral parents. The decays allowed by the neutral vee topology were $\Lambda \rightarrow p \pi^{-}, K^{0} \rightarrow \pi^{+} \pi^{-}$, and $\bar{\Lambda} \rightarrow \bar{p} \pi^{+}$. The events were classified by their reconstructed rest mass $M$, where $M^{2}=m_{a}^{2}+m_{b}^{2}+2 E_{a} E_{b}-2 \vec{p}_{a} \cdot \vec{p}_{b}$. Here a denotes the higher-momentum track and $b$ the lower. The analysis required $M$ to be $<3 \sigma$ away from the known rest mass for it to be identified as a particular decay, where $\sigma$ was the uncertainty in the reconstructed mass. The sign of the higher momentum particle was used to separate $\Lambda$ and $\bar{\Lambda}$ 's. The yield of $\Lambda$ events from the F.C5 and $\Lambda$ triggers is shown in Table 3.1 above and from the $\Xi^{0}$ trigger on Table 3.2. There was a class of events which passed both the $\Lambda$ and $K^{0}$ invariant mass requirement. This ambiguity occurred in events where the ratio $\left|\vec{p}_{a}\right| /\left|\vec{p}_{b}\right|=6.33$. Previous experiments $[18,21]$ have shown that due to their relative production cross-sections, the majority of the ambiguous $\Lambda$ $/ K^{0}$ decays were $\Lambda$ 's, while for the ambiguous $\bar{\Lambda} / K^{0}$ decays, they were $K^{0}$ 's. These ambiguous decays were eliminated from the $\Lambda$ event sample, but they were kept in the $\Xi^{0}$ sample, as the subsequent $\Xi^{0}$ fit should have rejected the $K^{0}$ 's.

The distribution of reconstructed $\Lambda$ masses, peaked at the $\Lambda$ rest mass of 1116 $\mathrm{MeV} / \mathrm{c}^{2}$, is shown for $\Lambda$ events in Figures 3.6. The $\sigma$ of the distribution, indicating the mass resolution, was $2.3 \mathrm{MeV} / \mathrm{c}^{2}$. The $\Lambda$ 's from $\Xi^{0}$ events had a better mass resolution because of the lower average momentum of the daughter $\Lambda$ 's compared to $\Lambda$ 's from the target.

\subsubsection{Kinematic $\Lambda$ fit}

To improve the decay vertex resolution, a additional, more constrained fit to the events with an identified $\Lambda$ decay was made. This was done by re-fitting the wire hits under the $\Lambda \rightarrow p \pi^{-}$hypothesis. The additional assumption of the known particle rest masses 


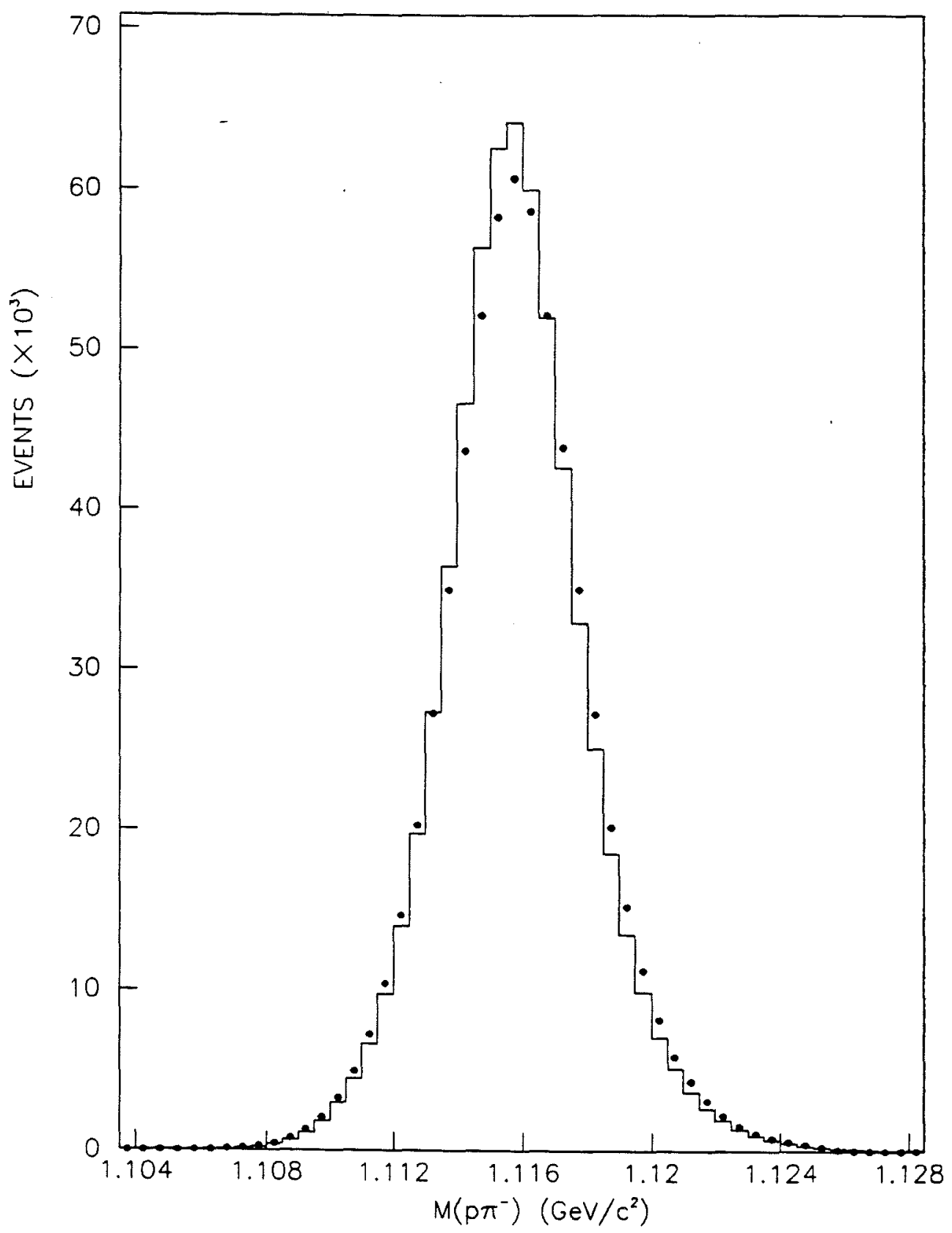

Figure 3.6: Invariant mass of beam $\Lambda$ events. The distribution is peaked at the $\Lambda$ rest mass of $1116 \mathrm{Mev} / \mathrm{c}^{2}$, with a $\sigma$ of $2.3 \mathrm{MeV} / \mathrm{c}^{2}$. Again, the real $\Lambda$ events are plotted as "dots", the MC events as the "histogram". 
added three constraints as elements of the total $\chi^{2}$. The decay vertex was optimized by moving it along $\mathrm{z}$ in $1 \mathrm{~cm}$ steps, re-doing the fit each time, until the minimum $\chi^{2}$ was realized. Analysis of Monte Carlo events revealed that this kinematic fit improved the average vertex z-resolution $\sigma$ from $75 \mathrm{~cm}$ to $50 \mathrm{~cm}$. This difference between this "kinematic" $\chi^{2}\left(\chi_{K}^{2}\right)$ and the existing geometric fit $\chi^{2}\left(\chi_{G}^{2}\right)$ is plotted for $\Lambda$ events in Figure 3.7. Only events with $\Delta\left(\chi_{K}^{2}-\chi_{G}^{2}\right)<10$ were kept, in order to eliminate poorly-reconstructed events. This eliminated only $2 \%$ of the $\Lambda$ events.

\subsection{Beam $\Lambda$ Events}

A beam $\Lambda$ event was defined as one where the $\Lambda$ was produced in one of the targets and travelled through the collimator into the decay volume without scattering off the walls of the collimator. This sample of events served two purposes. The first was as the data for the $\Lambda$ lifetime fits. The second was to define the neutral beam axes to be used in the $\Xi^{0}$ fits.

The backgrounds to be eliminated were $\Lambda$ 's produced in the collimator, and those which came from $\Xi^{0}$ decays. These backgrounds were removed by requiring all beam $\Lambda$ candidates to have a decay vertex and momenta which fell within the angular phase space of events from the targets. As the $\Lambda$ data itself was used to establish the target positions and phase space, the selection criteria were applied in steps to ensure selfconsistent answers.

The first step was to identify which of the two beams a given event came from. This was accomplished by projecting the reconstructed $\Lambda$ momentum vector upstream from its decay vertex to the $z$-plane at the middle of the collimator. The events from the two targets were well-separated in $X_{\text {COLLIMATOR. Events with }} X_{\text {COLLIMATOR }}<-2.05$ cm were declared East beam events, while those greater than this were declared West beam events. 


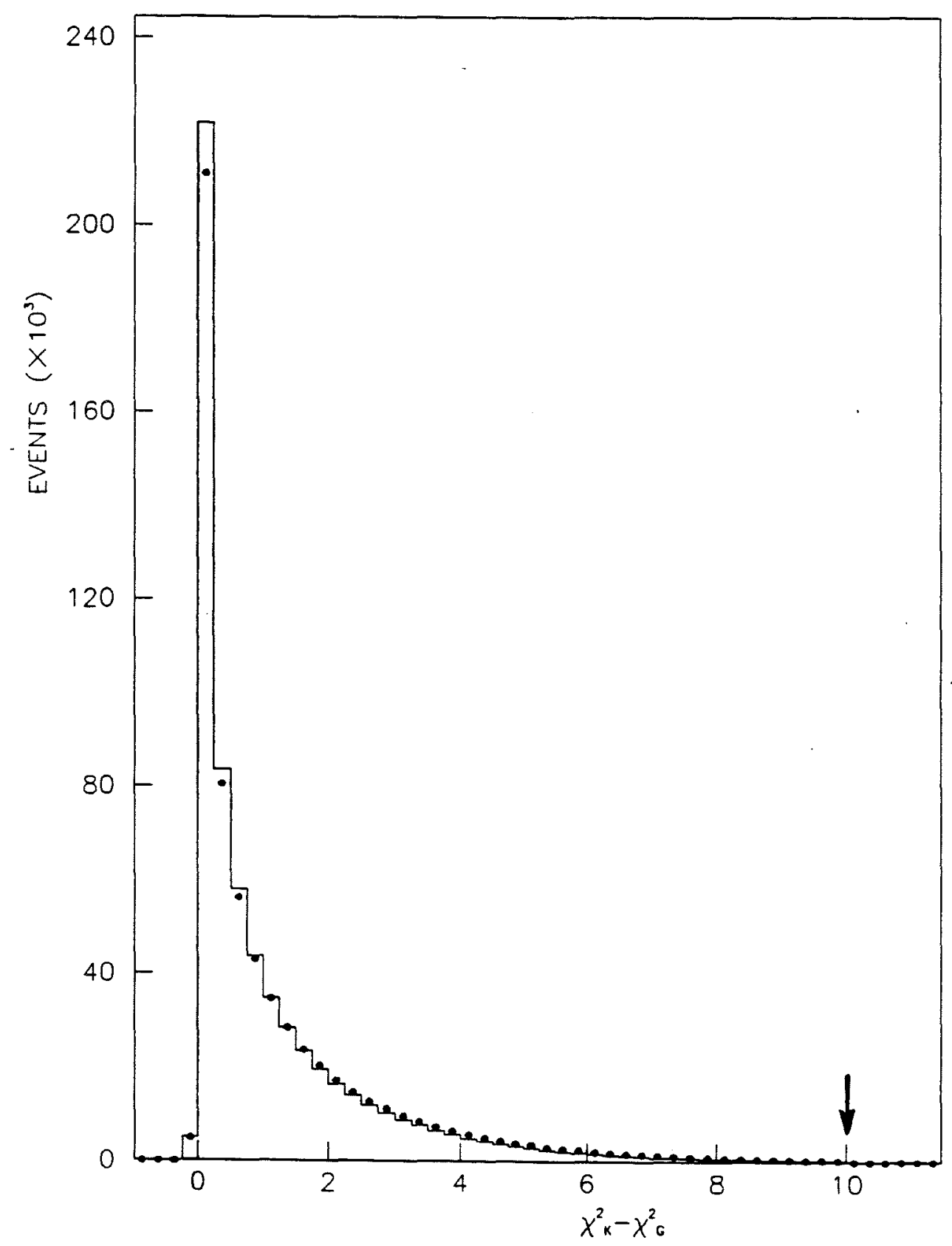

Figure 3.7: $\Delta\left(\chi_{K}^{2}-\chi_{G}^{2}\right)$ for $\Lambda$ events. Only events with $\Delta\left(\chi_{K}^{2}-\chi_{G}^{2}\right)<10$ were used. Again, the real $\Lambda$ events are the "dots", the MC events are the "histogram". 
All $\Lambda$ events from each target for a run were then summed together to give an initial measurement of the axis of the neutral beam in each run. The $\Lambda$ momenta were projected back upstream from the decay vertex to the $z$-plane of the target to define the average $\mathrm{x}$ and $\mathrm{y}$ at the target. The average beam angles $\Theta_{x}$ and $\Theta_{y}$, defined respectively as the momentum ratios $P_{x} / P_{z}$ and $P_{y} / P_{z}$ of the fitted $\Lambda$, completed the definition of the neutral beam axis.

Selection criteria based on the deviation of $\Lambda$ 's from the neutral beam axis were used to identify the beam $\Lambda$ events. First, the square of the distance between the projected position of the momentum vector of an event and the center of the neutral beam at the target plane was calculated. The true beam $\Lambda$ 's had a narrow'distribution peaked at zero in this " $R^{2}$ " quantity, while the collimator-produced and daughter $\Lambda$ 's had a very broad distribution in $R^{2}$. Initial requirements for beam $\Lambda$ 's were $R^{2}<0.45 \mathrm{~cm}^{2}$ and $R^{2}<1.50 \mathrm{~cm}^{2}$, for the downstream and upstream targets respectively. The neutral beam axis was re-calculated using those events which passed these criteria. The $R^{2}$ distributions for beam $\Lambda$ candidates are shown in Figure 3.8. For the downstream target events, the final criterion was $R^{2}<0.25 \mathrm{~cm}^{2}$. For upstream target events, where the beam and daughter $\Lambda R^{2}$ distributions were clearly separated, the criterion was $R^{2}<0.90 \mathrm{~cm}^{2}$.

The next stage of analysis of the $\Lambda$ data added requirements on the beam angles as well as the final $R^{2}$ requirements. Histograms of the difference $\left(\Delta \Theta_{x}\right.$ and $\left.\Delta \Theta_{y}\right)$ between event angles and the averages beam angles were used to reveal the remaining backgrounds and to choose the maximum allowed $\Delta \Theta_{x}$ and $\Delta \Theta_{y}$. These criteria were checked by comparing the real event distributions to those from MC beam $\Lambda$ events. Figure 3.9 shows the distributions and indicates the requirements for DE events. These histograms have all beam $\Lambda$ selection criteria applied except that using the quantity being plotted, so that the remaining background can be seen. The final criteria separated by target are shown in Table 3.4. The upstream targets had a wider $R^{2}$ range 


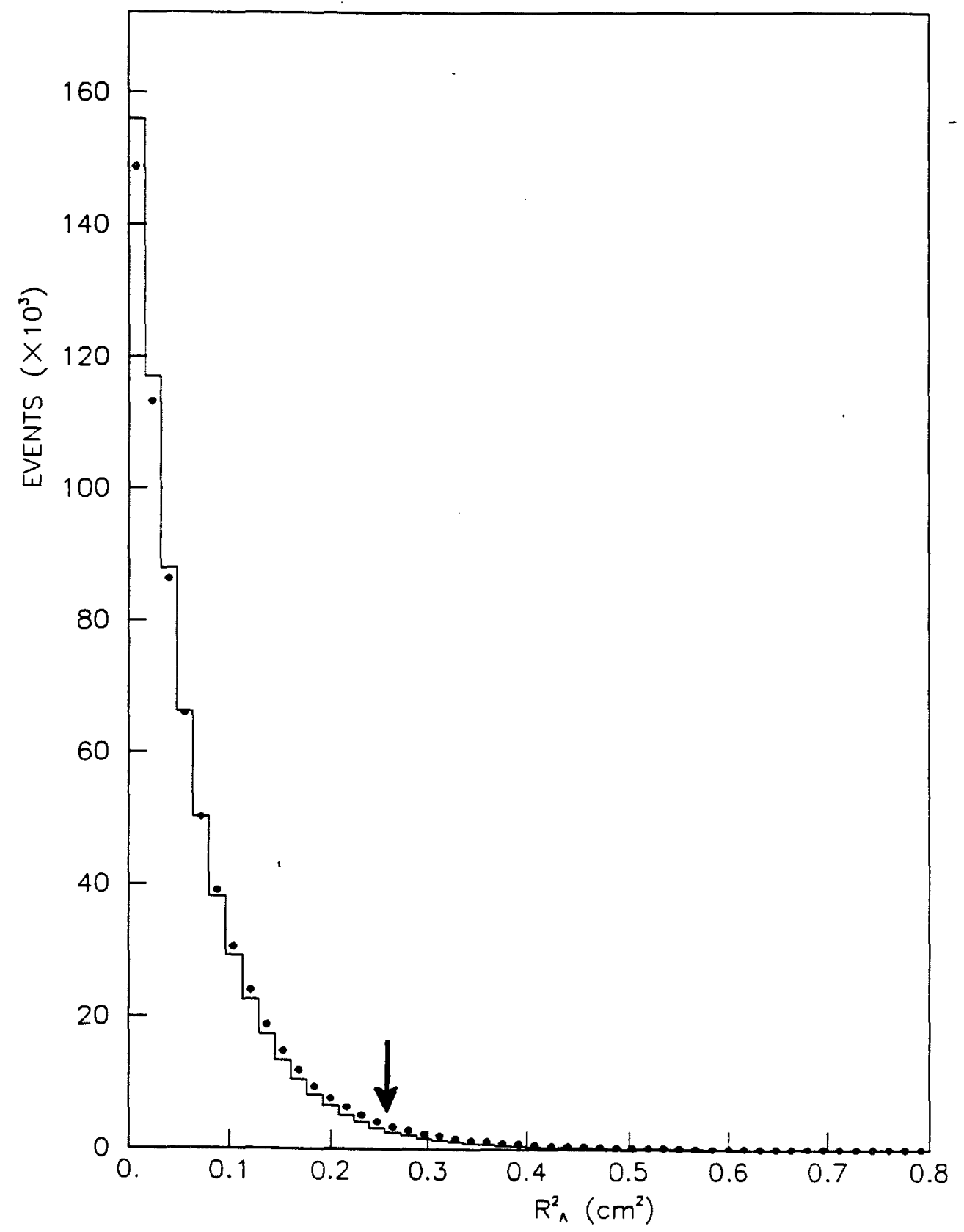

Figure 3.8: $R^{2}$ of beam $\Lambda$ 's from the downstream targets. Only $\Lambda$ decays with $R_{\Lambda}^{2}$ $<0.25 \mathrm{~cm}^{2}$ were used in the beam $\Lambda$ sample. (Data - dots , MC - histogram) 

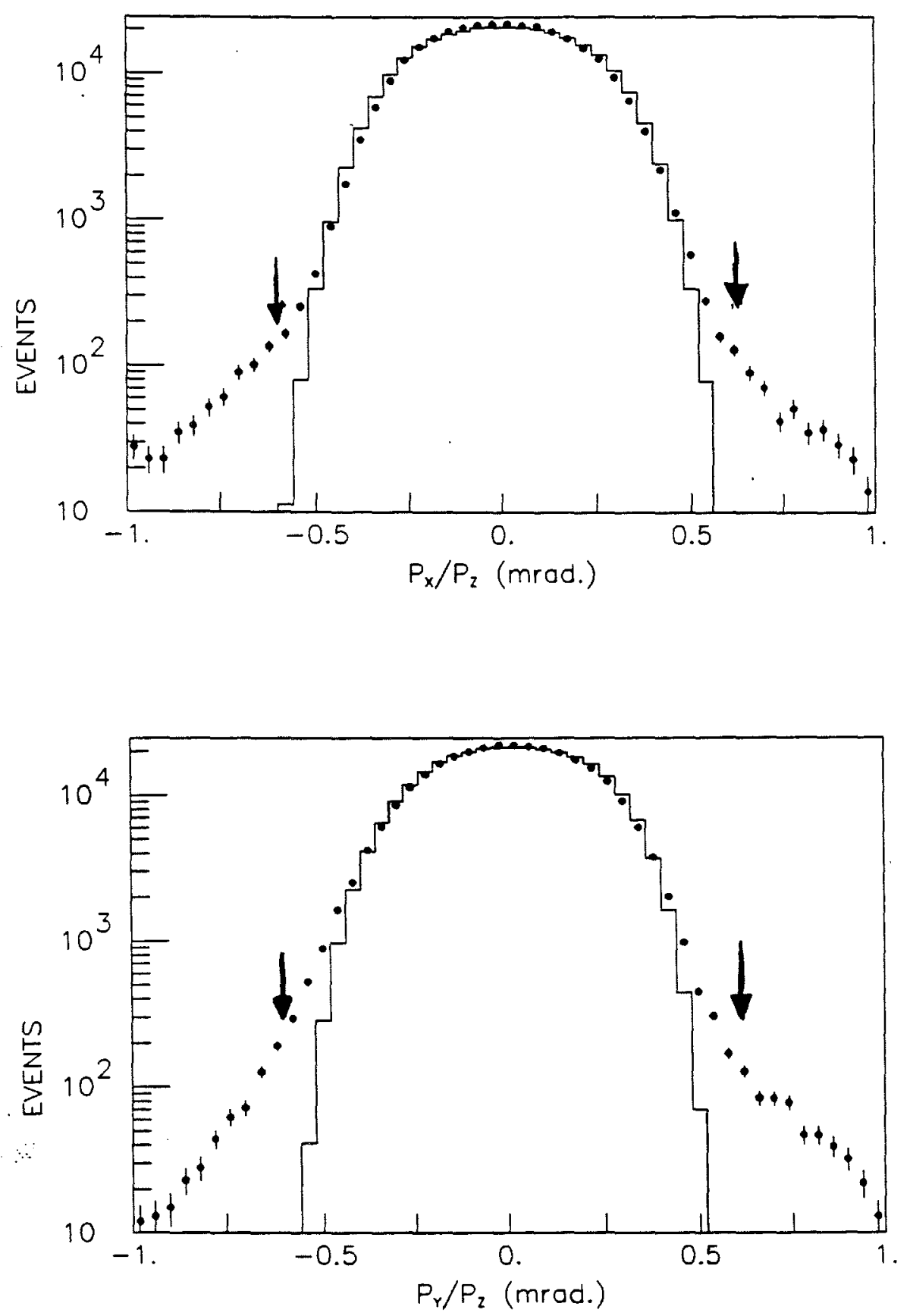

Figure 3.9: $\Delta \Theta_{x}$ and $\Delta \Theta_{y}$ distributions for $D E$ target $\Lambda$ events. The selection criteria used to identify beam $\Lambda$ 's are shown by the arrows. (Data - dots , MC - histogram) 


\begin{tabular}{|c|c|c|c|c|c|}
\hline \multirow{2}{*}{$\begin{array}{l}\text { Beam } \Lambda \\
\text { Quantity }\end{array}$} & \multirow[b]{2}{*}{ Units } & \multicolumn{4}{|c|}{ Target } \\
\hline & & $\mathrm{DE}$ & $\overline{\mathrm{UE}}$ & $\overline{\mathrm{DW}}$ & UW \\
\hline$R^{2}$ & $\mathrm{~cm}^{2}$ & $<0.25$ & $<0.90$ & $<0.25$ & $<0.90$ \\
\hline$\left|\Delta \Theta_{x}\right|$ & mrad. & $<0.600$ & $<0.275$ & $<0.625$ & $<0.300$ \\
\hline$\left|\Delta \Theta_{y}\right|$ & mrad. & $<0.600$ & $<0.175$ & $<0.700$ & $<0.200$ \\
\hline
\end{tabular}

Table 3.4: Final beam $\Lambda$ criteria separated by target

both because that target was much farther from the detector, which meant the projected position had a larger uncertainty due to the finite resolution, and because of the greater width of the parent proton beam. The upstream beams had tighter beam angle requirement because of the greater distance between that target and the collimator resulted in a narrower beam in the apparatus. Differences between East and West beam angle criteria were due mainly to differences in the parent proton beam shapes, with some effect from the different collimator tilts.

In Figures 3.10 and 3.11 are shown the momentum and decay vertex $\mathrm{z}$ distributions for the downstream(DS) target beam $\Lambda$ events from the F.C5 trigger compared to the MC events. The momentum distribution was cut off at the low end by the low acceptance for low-momentum $\pi^{-}$'s. The decay vertex distribution clearly shows the effect of the trigger counters V1 and DK, whose z-positions are indicated. Figures 3.12 and 3.13 are for the downstream target beam $\Lambda$ 's events from the $\Lambda$ trigger. There were fewer high-momentum events in this sample due to the low acceptance of the proton counters for high-momentum particles. These plots show the distributions to be used in measuring the $\Lambda$ lifetime. Events whose acceptance was sensitive to the locations of the V1 and DK counters and to the modelling of the vertex resolution were removed from the final samples by requiring the $Z$ of the decay vertex to lie between 200 . and 1900. $\mathrm{cm}$ downstream of the end of the collimator. These restrictions are indicated on the $\Lambda$ decay vertex plots. 


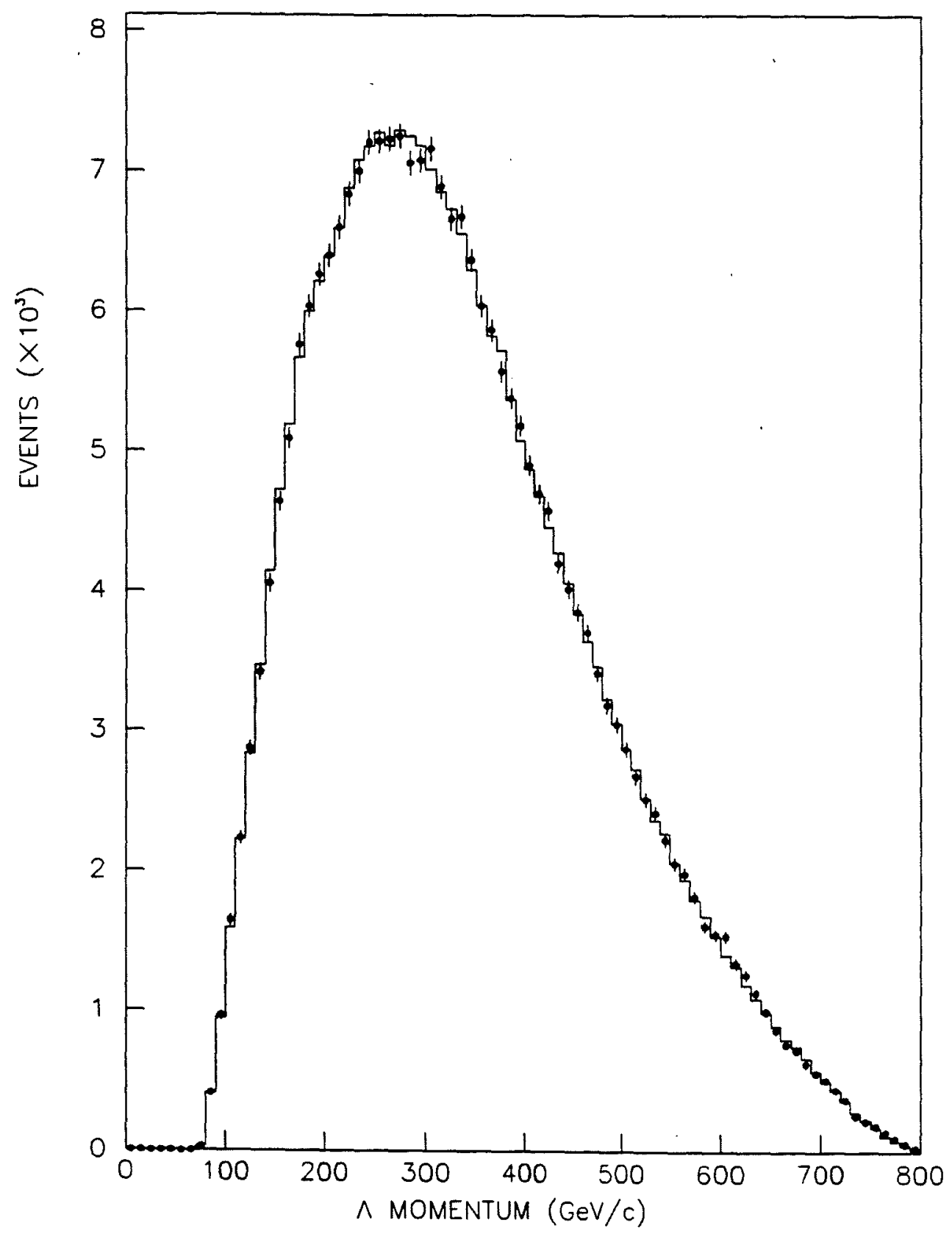

Figure 3.10: Momentum of DS beam $\Lambda$ events - F.C5 trigger. (Data - dots , MC histogram) 


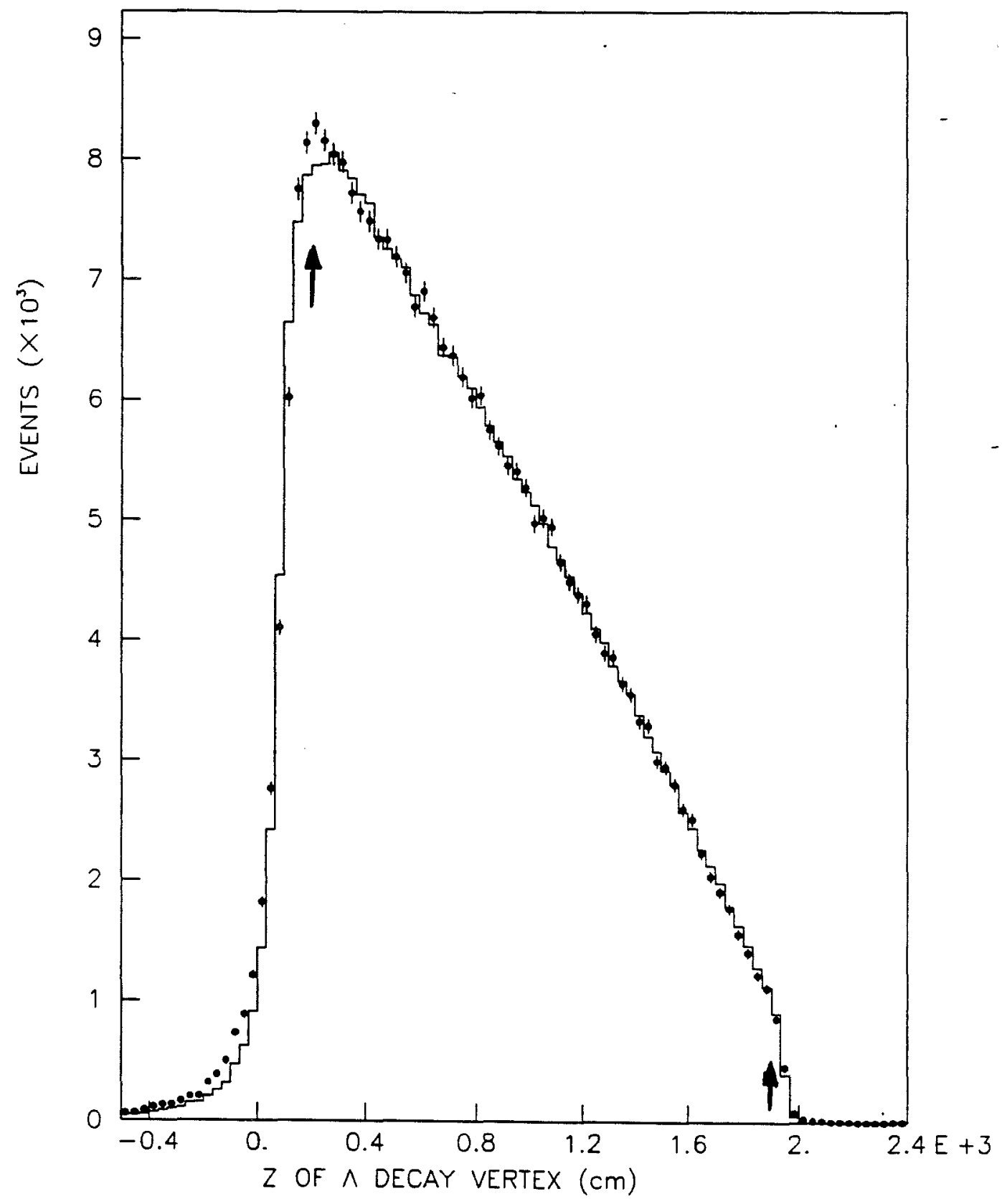

Figure 3.11: Decay vertex $\mathrm{Z}$ of DS beam $\Lambda$ events - F.C5 trigger. The allowed region for $\Lambda$ decays from 200. to 1900. $\mathrm{cm}$ is shown by the arrows. (Data - dots , MC histogram) 


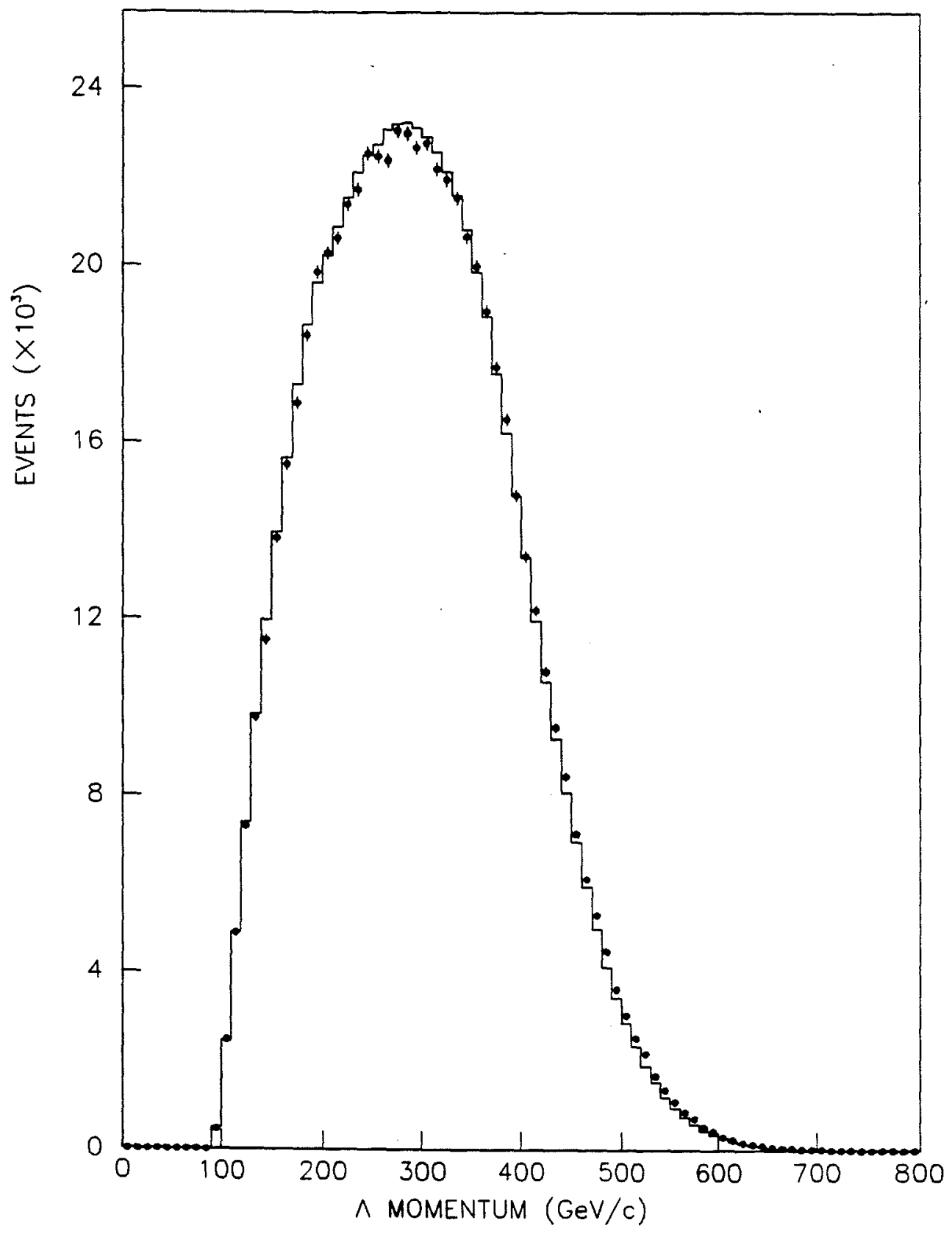

Figure 3.12: Momentum of DS beam $\Lambda$ events - $\Lambda$ trigger. (Data - dots, MC - histogram) 


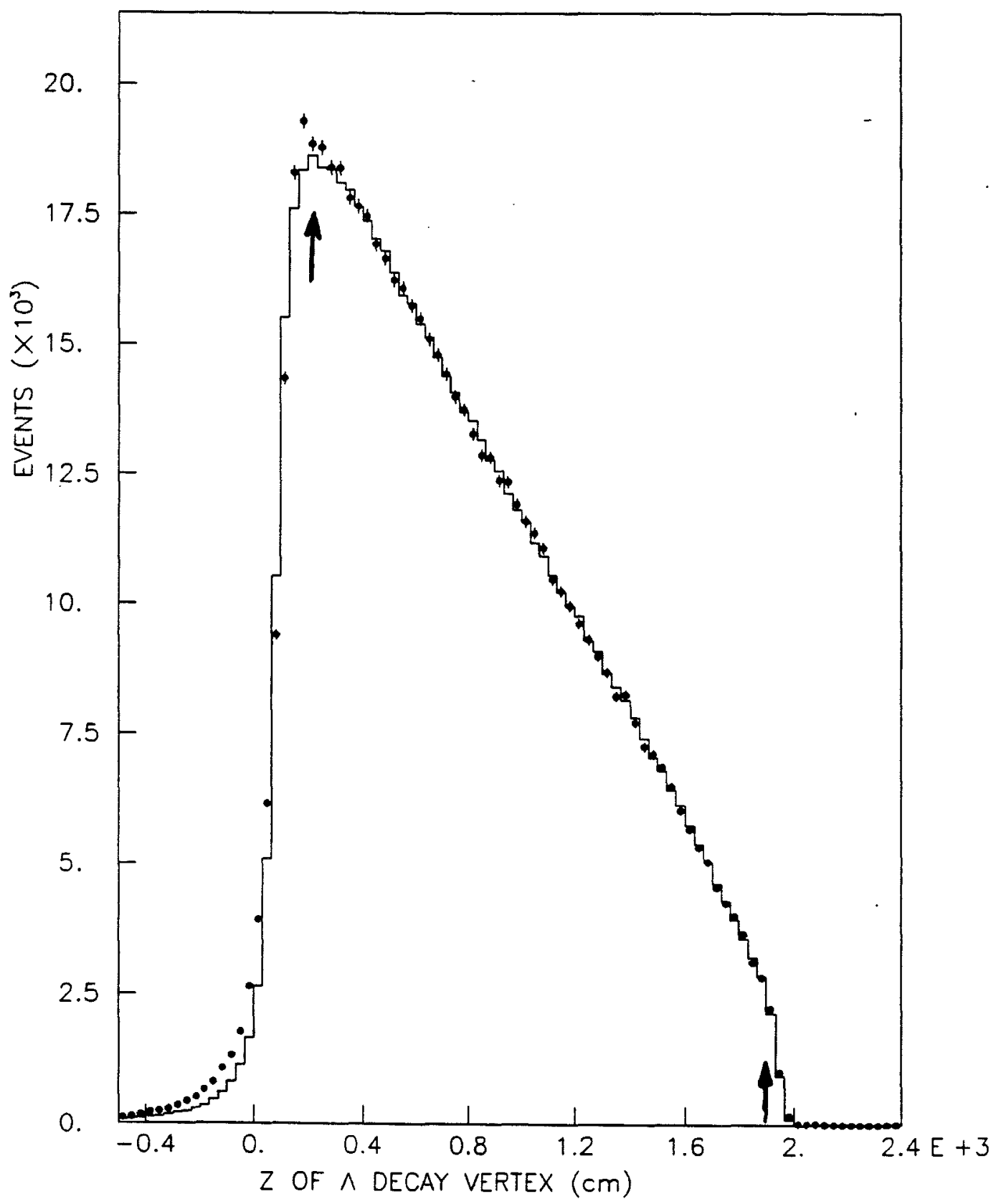

Figure 3.13: Decay vertex $\mathrm{Z}$ of DS beam $\Lambda$ events - $\Lambda$ trigger. The allowed region from 200. to 1900. cm is shown.(Data - dots , MC - histogram) 


\subsection{Photon Reconstruction}

After the $\Lambda$ reconstruction and fits were completed, the lead glass data were analyzed for events from the $\Xi^{0}$ trigger. Events from other triggers were also analyzed in order to determine the efficiency of the photon trigger. The ADC and TDC signals from the blocks were used find candidate photon shower clusters. Selection criteria were used to separate the photon showers from those created by charged hadrons. The energy and position of each cluster were calculated. Events which had at least two photon clusters in them underwent a preliminary $\pi^{0}$ fit and were passed on to the $\Xi^{0}$ reconstruction.

\subsubsection{Cluster Selection}

Before forming the lead glass data into clusters, the ADC and TDC data for each block were examined. Only blocks with an ADC signal above a threshold value and with TDC signals indicating that its timing was within one RF bucket (18 ns) of the trigger were included in the clustering.

An ADC threshold was imposed for several reasons. The first was to reject very small ( $\sim 5$ counts) signals caused by imperfect pedestal subtraction and noise. Another was that small signals that fell below the threshold of the discriminator to the low-threshold (regular) TDCs would lack timing information. The threshold level in terms of ADC counts was established by analyzing raw event records and plotting the probability of a valid TDC signal as a function of ADC counts. The resulting error function distribution showed a $50 \%$ efficiency level at 15 ADC counts. Even for signals above this TDC level there was an advantage in rejecting them. This was because clusters could have blocks on their edges which did not have a significant amount of the energy of the cluster, but were adjacent to the edge of another cluster. By not considering these blocks when forming clusters, the yield of separate clusters would be increased. If the threshold was too high, it would decrease the energy and position resolution. An ADC threshold of 20 counts was finally established as a reasonable compromise. 
Both the high-threshold ( $\gamma$ trigger) and low-threshold TDC's were used for the signal timing. For the inner blocks in the $\gamma$ trigger, the trigger TDC data was checked first. Such blocks were used in the clustering if the trigger TDC was non-zero and within 20 ns of the average TDC value for that block. The averages were established for every run by examining the distribution of all TDC's from a block for all events and finding the peak. The $<20 \mathrm{~ns}$ requirement was determined by studying TDC distributions for reconstructed $\Xi^{0}$ 's, with its width determined by the jitter in the timing due to the finite trigger overlap time and different slew rates for lead glass signals at the input to the discriminators. The trigger TDC was checked first because it was less likely to have a false value or be zero due to being held off by an earlier signal. This was because the TDC's would ignore new inputs for $250 \mathrm{~ns}$ after receiving an input. For blocks not in the trigger, or if the trigger TDC was zero, the low TDC data was checked. If the low TDC was non-zero and within $20 \mathrm{~ns}$ of the average TDC time, then the block was used in the clustering.

After these ADC and TDC checks, those blocks which were found to have good signals were then arranged in clusters of adjacent blocks, with no restriction on the size of the cluster. For a cluster, each block's ADC signal was multiplied by the gain constants from the $e^{+} e^{-}$runs to determine the cluster energy and the energy distribution used to determine the cluster center (See Section 2.8.2)

\subsubsection{Photon Cluster Identification}

All of the clusters found in the event were checked against various photon shower criteria. A major problem was that the relatively high-momentum protons from the $\Lambda$ decays always struck the array, unlike the pions from the $K^{0} \rightarrow \pi^{+} \pi^{-} \pi^{0}$ decays, which usually had a low enough momentum to be swept off the array by the analysis magnets. The protons, and the $\pi^{-}$'s which struck the array, either passed through the blocks, leaving a small signal due to Cherenkov light, or they underwent a strong interaction, 
leaving a large extended cluster due to the hadronic shower. Several strategies were employed to reject these hadron shower clusters. If the center of a cluster was within $15 \mathrm{~cm}$ (equal to one-and-a-half block widths) of the projected track of a charged particle, or if the center of the cluster was in a block which was vetoed by the $\pi$-killer, then the cluster was eliminated as a candidate. If a block in the cluster had a signal which saturated the range of the ADC (4096 counts), which could happen due to the large amount of light produced in hadronic showers, the cluster was also removed from the list.

Photon clusters tend to be small and compact in lateral extent, taking up only a few blocks, (Figure 3.14), while hadronic showers can be very extended. Clusters with more than 7 blocks or which had an energy distribution which was not sharply peaked at the center blocks were removed. Requiring $>1 \mathrm{GeV}$ in the cluster eliminated those clusters caused by very low energy photons which did not come from $\pi^{0}$ decays.

Those clusters passing the photon criteria were then separated according to the location of their reconstructed centers. If the cluster was within $5.6 \mathrm{~cm}$ of the outer edge of the array, it was defined as an "edge" clusters. If they were within $5.6 \mathrm{~cm}$ of the edge of the hole in the middle of the array, they were defined as "hole" clusters. The energy of the hole cluster was then adjusted using the hole block gain constants (Section 2.25). Those clusters not near the edge or hole were termed regular or "nonhole" clusters. Only events with two and only two photon clusters were retained in the final $\Xi^{0}$ sample.

The beam $\Lambda$ events were analyzed for the presence of photon clusters. As shown in Figure 3.15, only $5 \%$ of the beam $\Lambda$ events had even one photon cluster. To further eliminate any background due to $\Xi^{0}$ decays, the events in the final beam $\Lambda$ samples were required to have no photon clusters in them. 


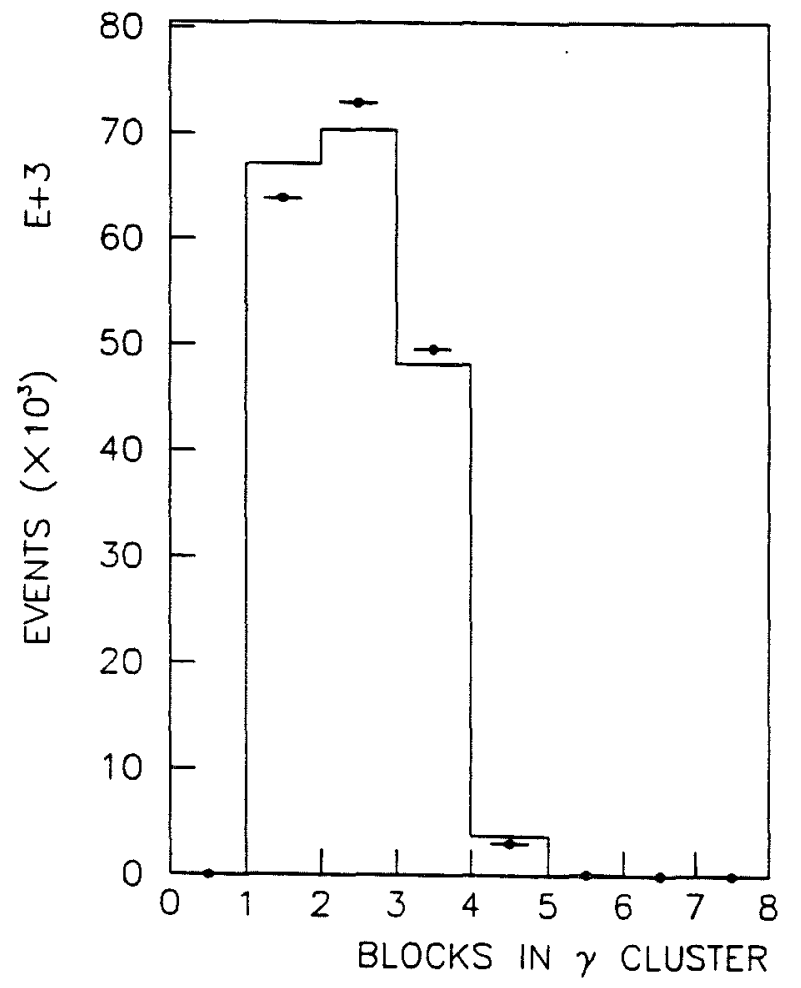

Figure 3.14: Number of blocks in photon showers for $\Xi^{0}$ events. (Data - dots , MC histogram) 


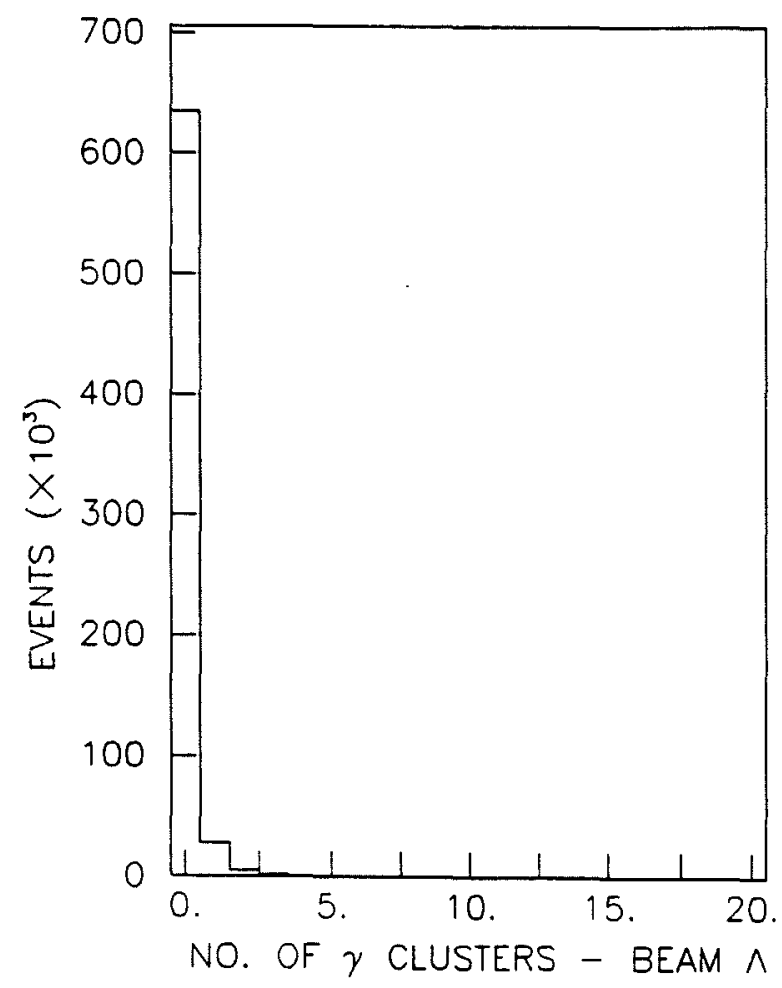

Figure 3.15: Number of photon clusters in beam $\Lambda$ events. Only events with no photon clusters were used. 


\section{$3.5 \quad \Xi^{0}$ Reconstruction}

\subsection{1 $\Xi^{0}$ Fit}

The $\Xi^{0}$ fit $[16,20]$ used kinematic constraints to determine the $\Xi^{0}$ decay vertex and momenta from the reconstructed $\Lambda$ and photon parameters. The fit required that the $\Xi^{0}$ vertex be within the volume formed by the intersection of the projected $\Lambda$ momentum vector and the neutral beam. The fit also constrained the reconstructed $\Xi^{0}$ momentum to project along the neutral beam back to the collimator and target. The neutral beam was defined by the neutral beam axis (Section 3.2 ) positions at the target and collimator, with its width determined by the size of the physical apertures at the target and collimator. Each of these constraints were used as elements in the overall $\chi^{2}$ of the fit.

The initial $\Xi^{0}$ vertex for the fit was found by projecting the $\Lambda$ momentum to the z-plane which was 0.4 times the distance from the exit of the defining section of the collimator to the $\Lambda$ decay vertex. The fit then forced the $\Xi^{0}, \Lambda$ and $\pi^{0}$ masses, and varied the measured $\Lambda$ momenta and photon parameters within their uncertainties to minimize the overall $\chi^{2}$. It then moved the $\Xi^{0}$ along the projected $\Lambda$ path within the neutral bearn volume until the position with the smallest $\chi^{2}$ was found. The vertex was not permitted to be downstream of the $\Lambda$ vertex and the search was halted at the upstream end of the collimator $(z=-331.5 \mathrm{~cm})$. The fit then calculated the resulting $\Xi^{0}, \Lambda, \pi^{0}$ and daughter particle momenta.

The uncertainties in the $\Lambda$ momenta used to compute the $\Xi^{0}$ fit $\chi^{2}$ were those derived from the earlier kinematic fit. The uncertainties in the photon energies and positions were the resolutions measured with the $e^{+} e^{-}$data (Section 2.8.2). Gaussian uncertainties equivalent to the size of the physical target and collimator apertures were used for the neutral beam $\chi^{2}$ contributions. 


\subsubsection{Bootstrap $\pi^{0}$ Calibration}

The $\Xi^{0}$ fit could be performed using the positions of the photon clusters but without using the reconstructed photon energies as constraints. The fitted photon energies which resulted from this "1-constraint" fit were then used to correct the ADC gain constants on a run-to-run basis. This "bootstrap" procedure was necessary because the yellowing of the lead glass blocks over time, caused by the large neutral particle flux in the spectrometer, reduced the light which reached the photomultiplier, and hence the output signal. This required the gain constants to be raised in order to maintain the lead glass energy calibration.

More restrictive criteria were used to select the $\Xi^{0}$ events used to determine the gain constants to make sure that only true $\Xi^{0}$ decays were included. A requirement that $\chi_{\Xi}^{2}$ $<5$ was imposed. A requirement of $R_{\Lambda}^{2}>0.15 \mathrm{~cm}^{2}$ was made on downstream target events to reject beam $\Lambda$ events. The clusters were required to have an energy $>4 \mathrm{GeV}$ and to not be "edge" clusters, where the shower energy might not be fully contained.

To reduce the statistical errors in calculating the gain constants for a particular run, the events from three consecutive runs were added together. The new constants were calculated with the same matrix solution technique used for the $e^{+} e^{-}$runs data (Section 2.8.2). The 92 resulting gain constants for each run, one for each blocks plus six additional constants for use with hole clusters, were then grouped with those from the other runs that occurred between the same lead glass array re-stackings. The constants from this group of runs were smoothed by fitting them to straight lines which could vary linearly with the run number. This fit allowed for the increase with time of the gain constants, which changed most rapidly in the blocks next to the hole. For blocks with only a few events per run in them, the weighted average of the constants from all runs in the group was used. These low-statistics blocks were on the outer parts of the array and did not experience any significant yellowing during the experiment.

The resulting constants were then used as input to new $\Xi^{0}$ fits. The energies and 
positions of the clusters were recalculated, and the best pair for a $\pi^{0}$ re-selected if needed. This bootstrap procedure rapidly converged, requiring only a few iterations.

\subsection{3 $\Xi^{0}$ Events}

After the bootstrap calibration was complete, the $\Xi^{0}$ events were fit again, this time including the photon energies as constraints. The $\chi_{\Xi}^{2}$ distribution for events with a successful fit is shown in Figure 3.16. All events were required to have a $\chi_{\Xi}^{2}<40$. The $z$ positions of the reconstructed $\Xi^{0}$ decay vertices are shown in Figure 3.17. Initially, events were required to have $z_{\Xi}>-331 \mathrm{~cm}$ (the beginning of the defining section of the collimator) to eliminate events which had failed to find a suitable $\Xi^{0}$ decay vertex. After studying the MC and data $\Xi^{0}$ events further, it was determined to require events in the final $\Xi^{0}$ sample to have a vertex farther downstream than the exit of the collimator ( 0 $\mathrm{cm}$ ). The distribution of daughter $\Lambda$ decay vertices peaks towards the end of the decay volume, as is shown in Figure 3.18. The same requirement that the $\Lambda$ decay vertex lay between 200. and 1900. cm was made on these events as had been made on the beam $\Lambda$ events. The momentum spectrum of the daughter $\Lambda$ 's (Figure 3.19) is much softer than that of the beam $\Lambda$ 's.

The reconstructed $\Xi^{0}$ and $\pi^{0}$ rest masses were calculated using the $\Lambda$ momenta, photon energies and positions determined before the $\Xi^{0}$ fit together with the fitted $\Xi^{0}$ decay vertex. The pre-fit values were used because in the fit the $\Xi^{0}$ and $\pi^{0}$ rest masses had been forced to be equal to the known values. The $\pi^{0}$ mass resolution was about $13 \mathrm{MeV}$, as shown in Figure 3.20. This resolution was $12 \mathrm{MeV}$ for events where both photons were non-hole (a "non-hole $\pi^{0}$ ") and $14 \mathrm{MeV}$ for events with at least one photon next to the hole ( $a$ "hole $\pi^{0}$ ") (Figure 3.21). The mass resolution was worse for hole $\pi^{0}$ 's because of the greater energy and position uncertainty caused by the photon shower's leaking energy into the hole.

The $\Xi^{0}$ mass resolution (Figure 3.22 ) was $15 \mathrm{MeV}$, predominantly due to the $\pi^{0}$ mass 


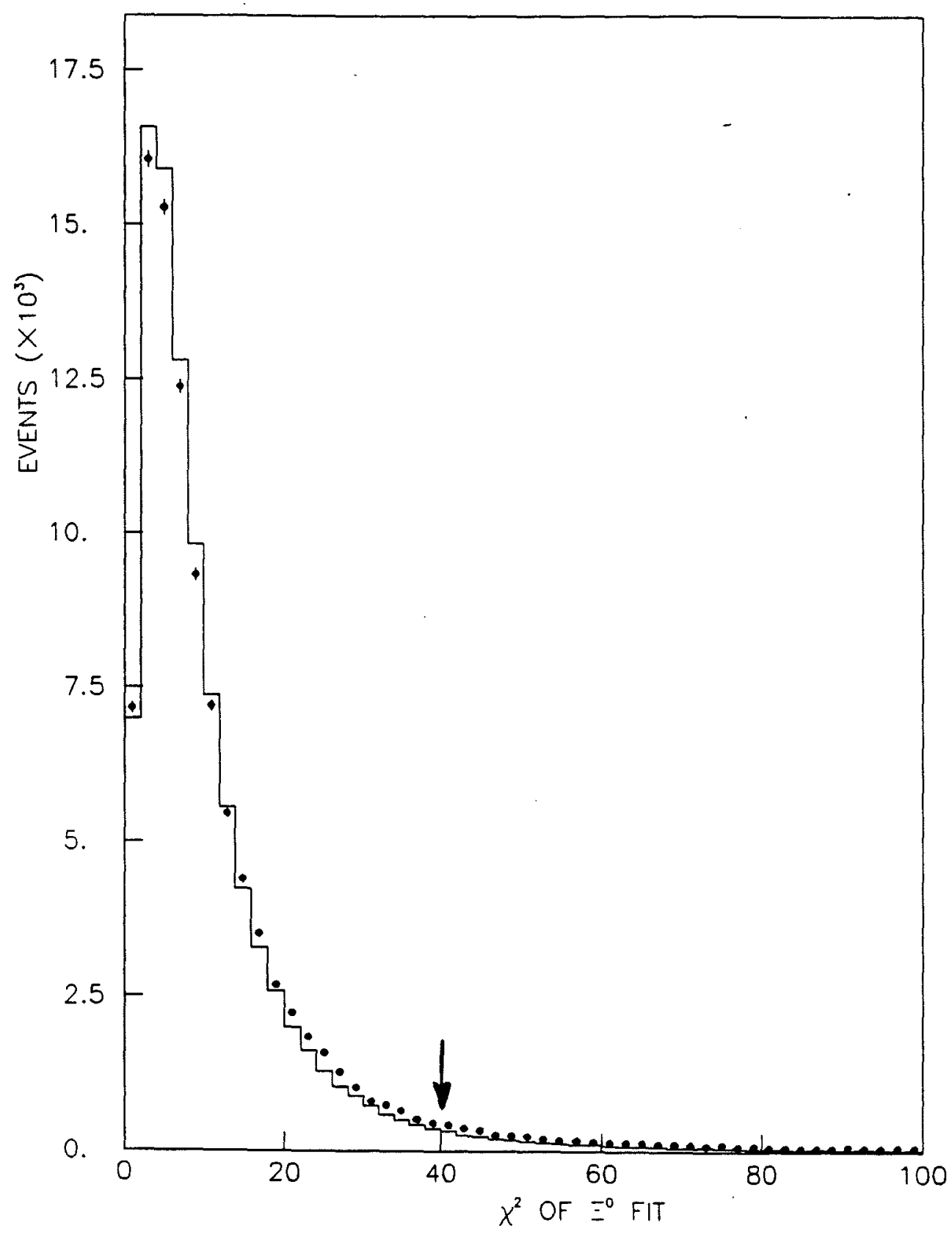

Figure 3.16: $\chi^{2}$ distribution for $\Xi^{0}$ fit. Only events with $\chi_{\Xi}^{2}<40$ were used.(Data dots , MC - histogram) 


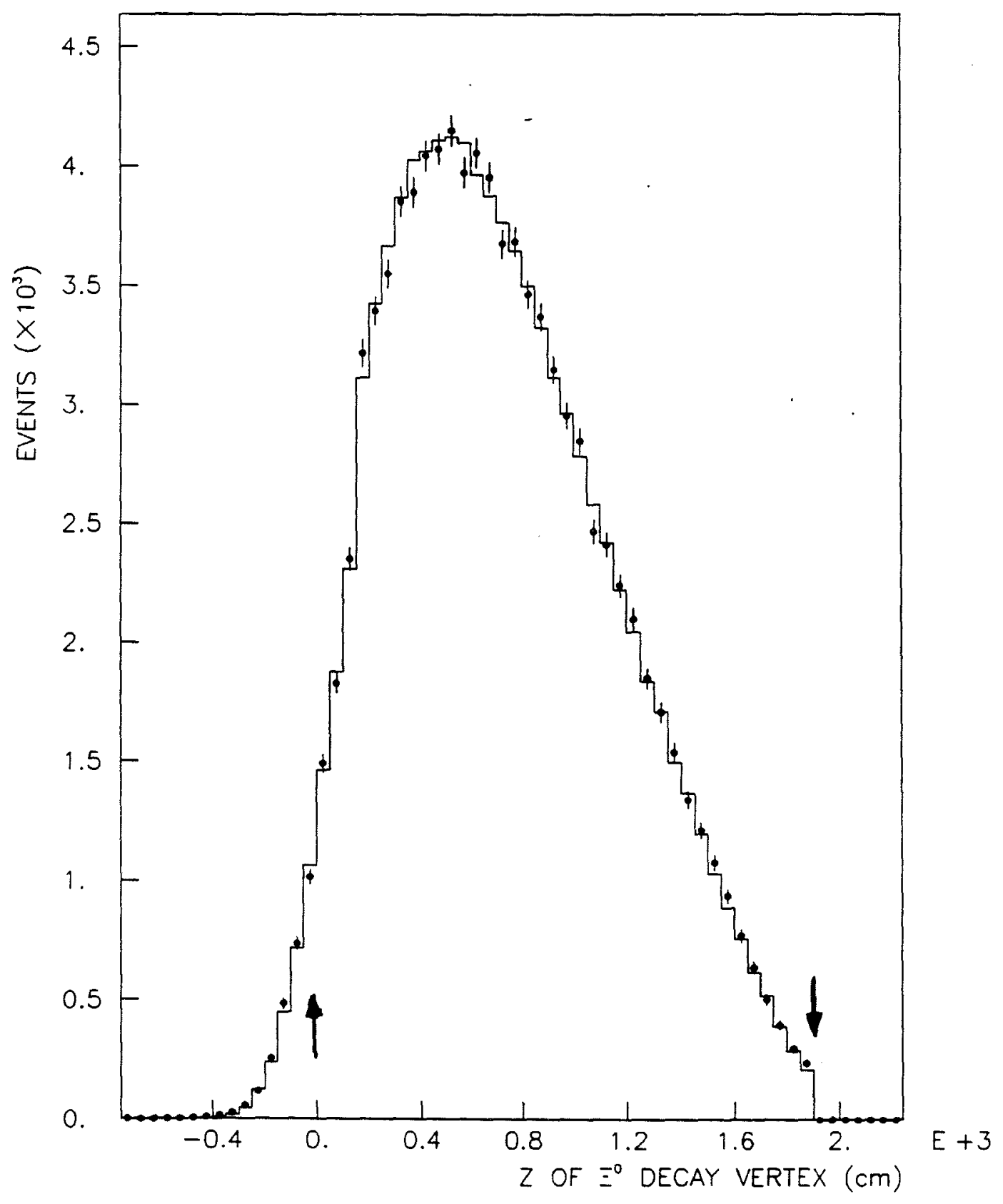

Figure 3.17: Distribution of reconstructed $\Xi^{0}$ decay vertices. $\Xi^{0}$ events were required to have a vertex with $\mathrm{z}$ between 0 . and 1900 . $\mathrm{cm}$ from the collimator. (Data - dots , MC - histogram) 


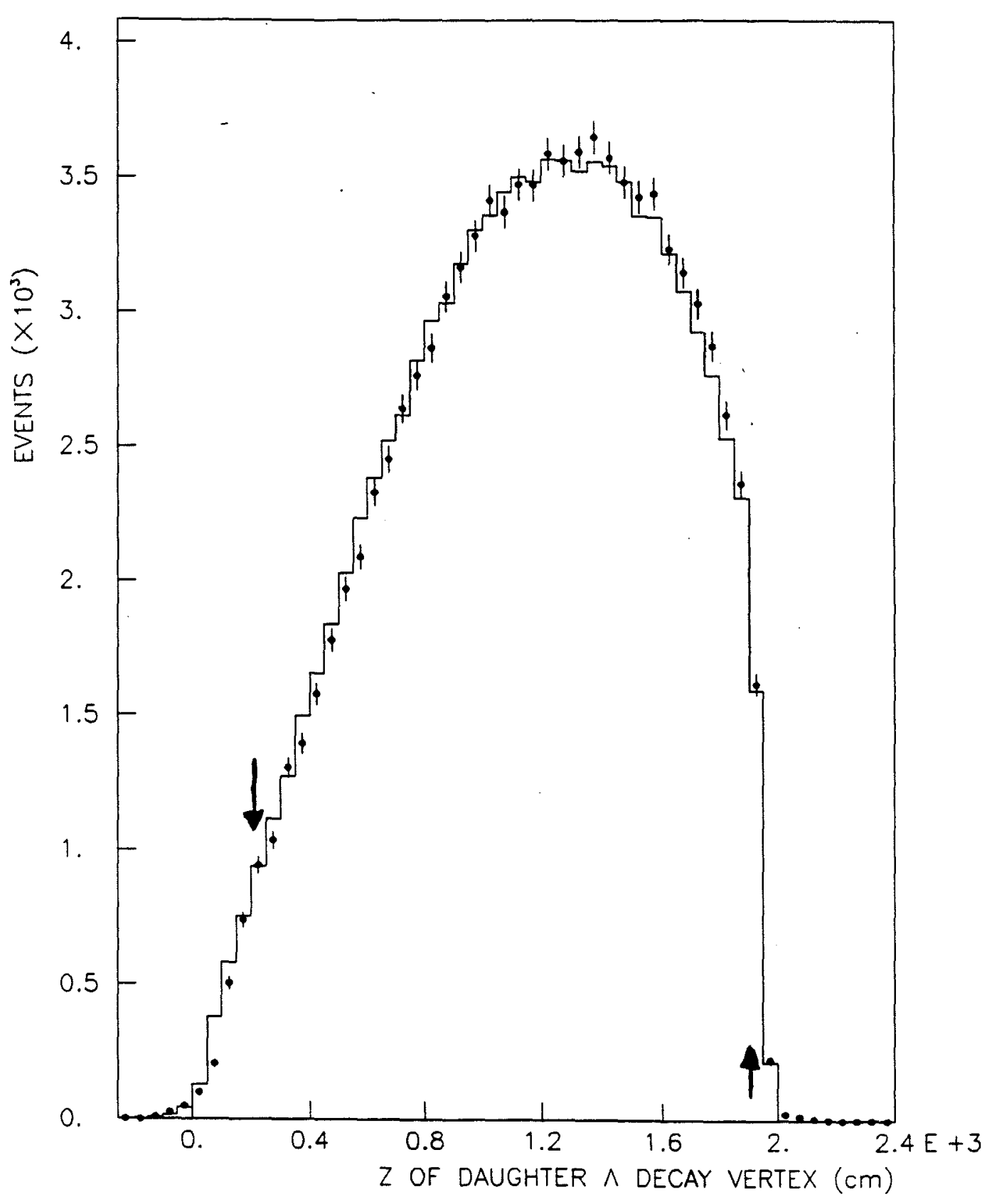

Figure 3.18: Distribution of daughter $\Lambda$ decay vertices for $\Xi^{0}$ events. Like the beam $\Lambda$ events, these were required to fall between 200 . and $1900 . \mathrm{cm}$ downstream of the collimator. (Data - dots , MC - histogram) 


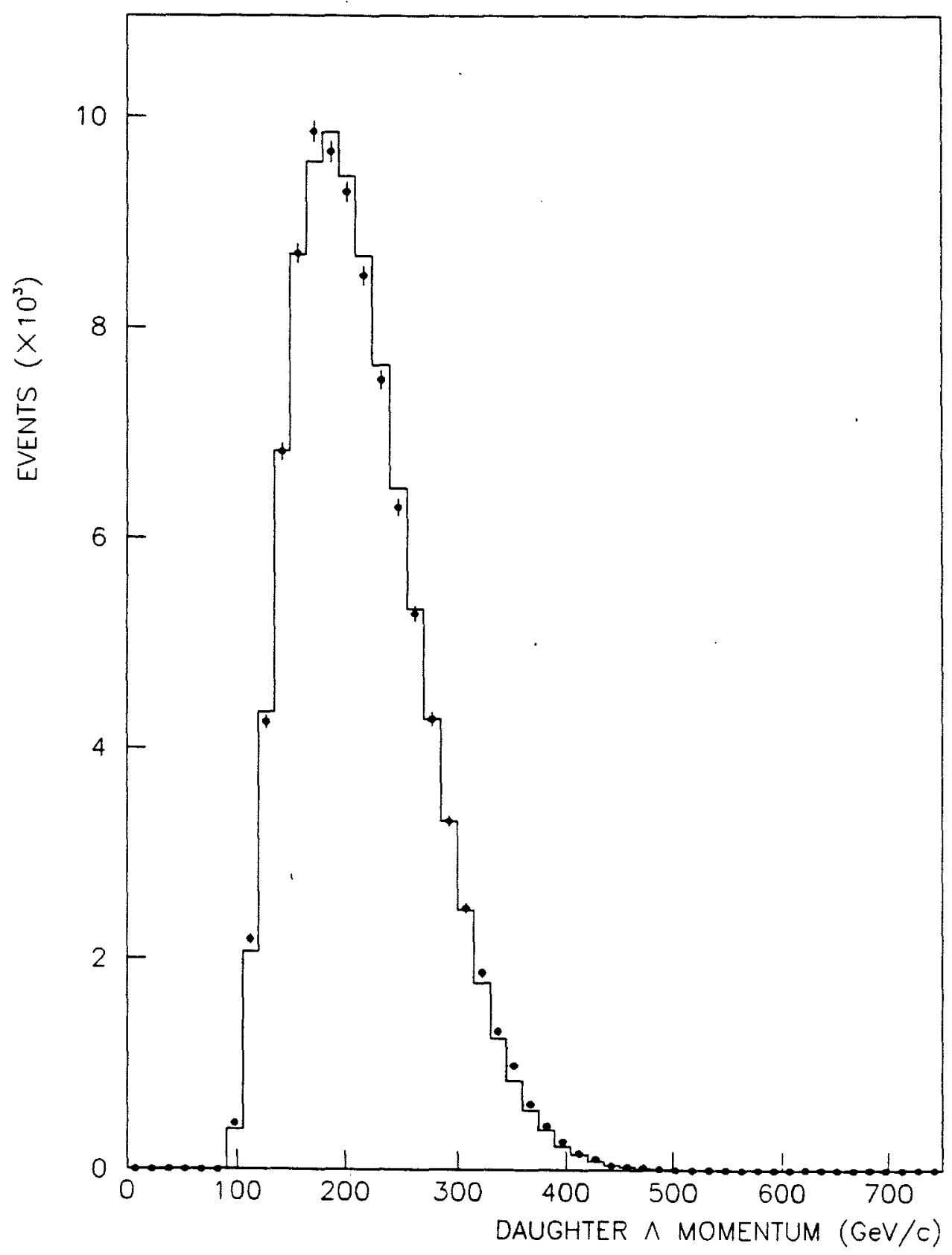

Figure 3.19: Momentum spectrum of daughter $\Lambda$ 's for $\Xi^{0}$ events. (Data - dots , MC histogram) 


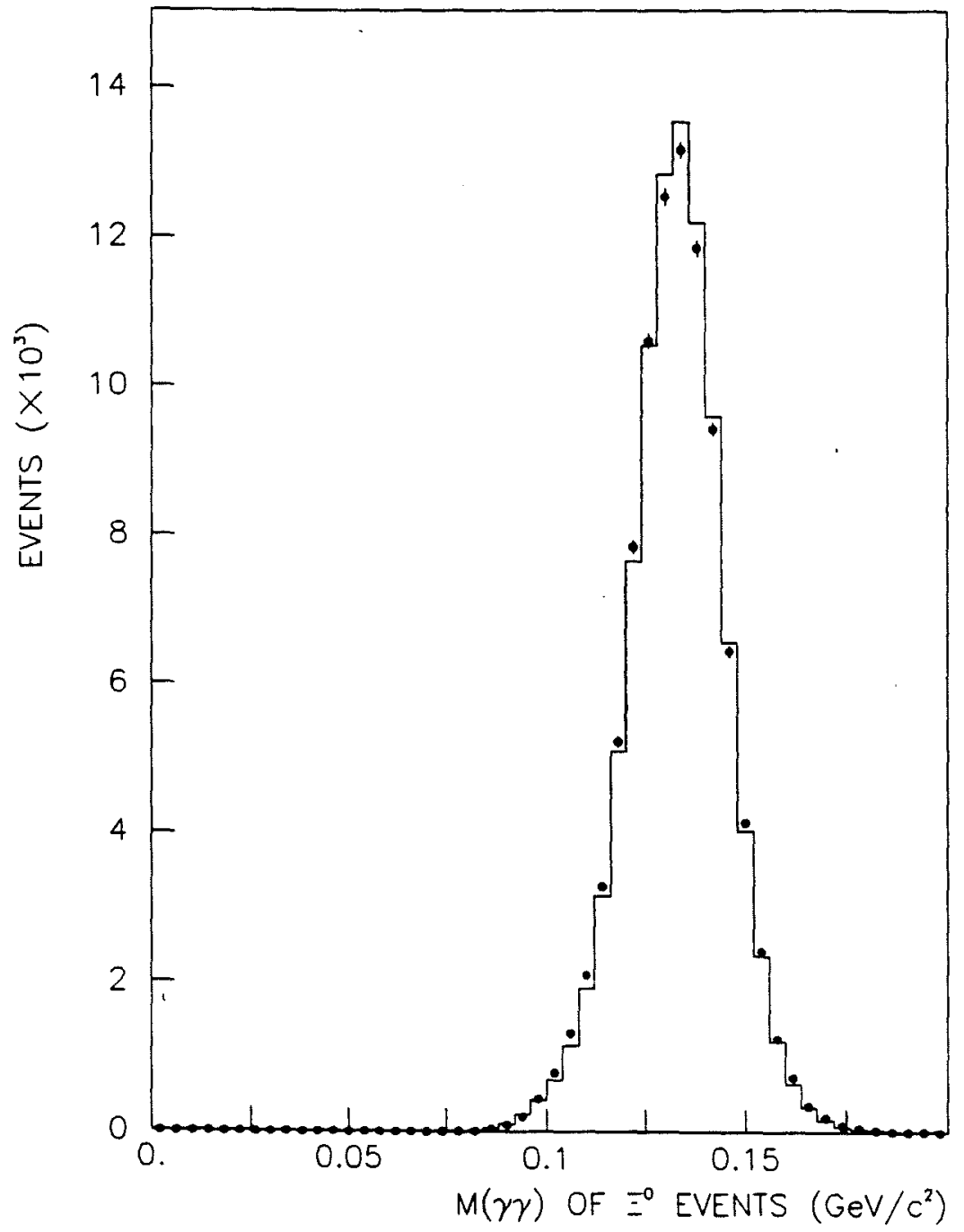

Figure 3.20: $\pi^{0}$ mass for accepted $\Xi^{0}$ events. (Data - dots , MC - histogram) 


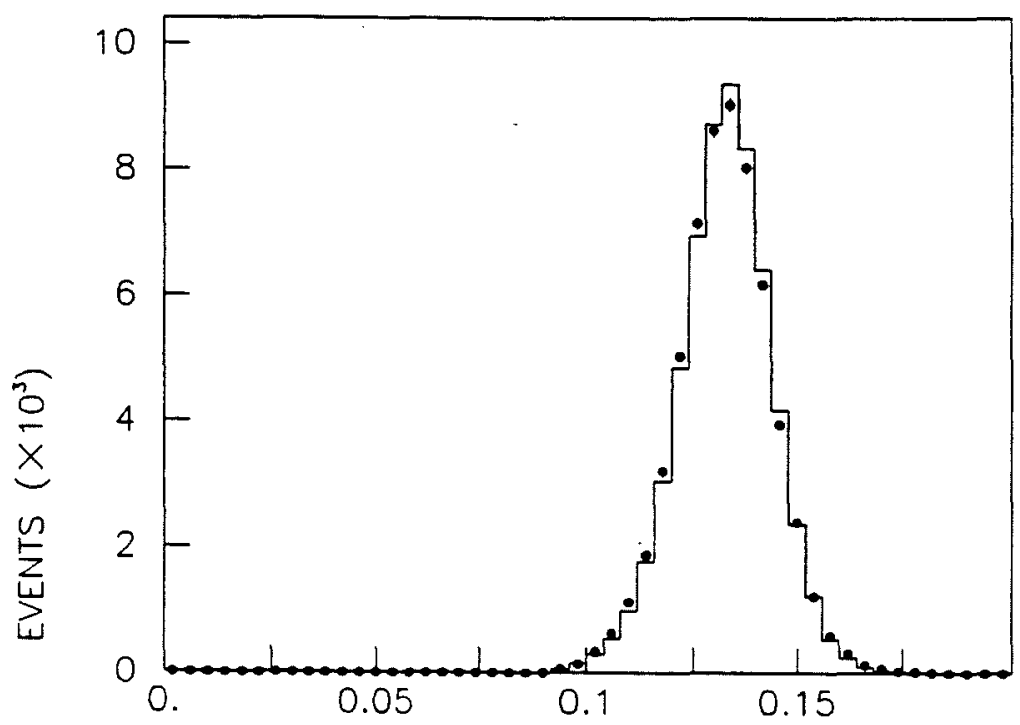

(a) $M(\gamma \gamma)-$ NON-HOLE EVENTS $\left(\mathrm{GeV} / \mathrm{c}^{2}\right)$

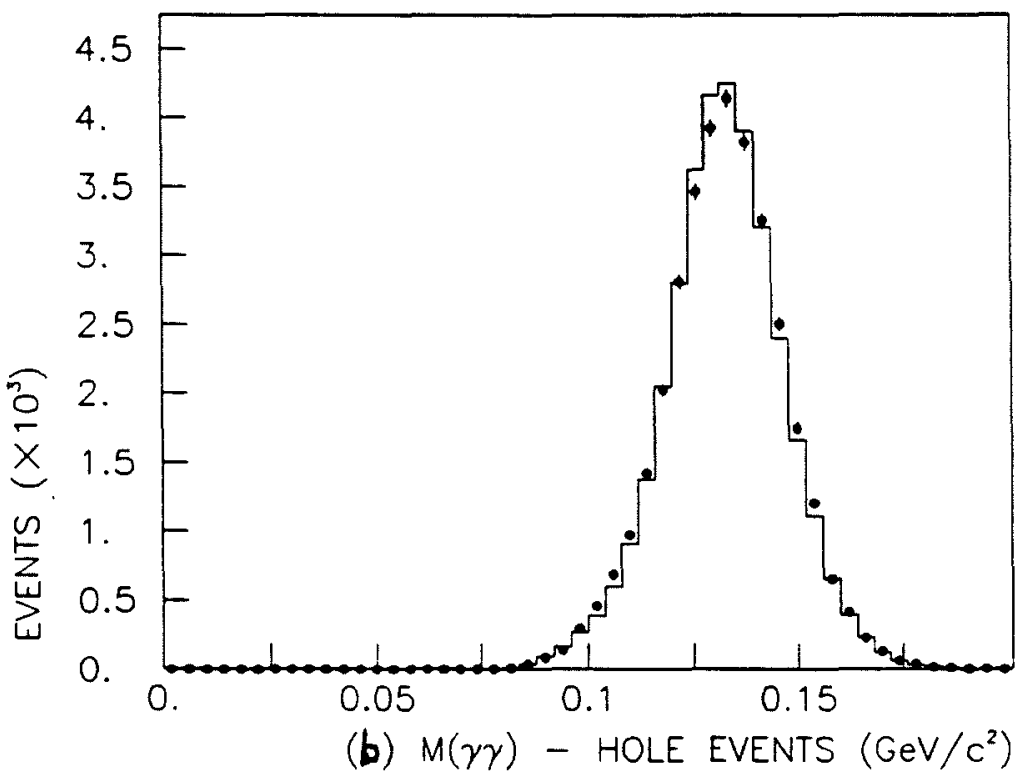

Figure 3.21: Reconstructed $\pi^{0}$ mass for (a) non-hole and (b) hole $\Xi^{0}$ event samples (Data - dots , MC - histogram) 
uncertainty. The momentum spectrum for accepted $\Xi^{0}$ events (Figure 3.23) was limited on the low-momentum end by the $\Lambda$ trigger acceptance and on the high-momentum end because of the low acceptance for high energy photons which passed through the hole in the lead glass array.

Further selection criteria were used on the $\Xi^{0}$ events to reduce contamination from background events due to beam $\Lambda$ 's and collimator-produced $\Xi^{0}$ 's. Events which had a reconstructed photon with energy below $5 \mathrm{GeV}$ had significantly larger average $\chi^{2}$, perhaps due to contamination from beam $\Lambda$ events, and were removed from the final sample. This requirement is indicated in the photon energy distributions for $\Xi^{0}$ events in Figure 3.24. The momentum vector of the fitted $\Xi^{0}$ was projected from its reconstructed vertex upstream to the plane of the target. The $R^{2}$ between this projected position and the neutral beam at the target was calculated (Figure 3.25) and events with $R_{\Xi}^{2}>0.35 \mathrm{~cm}^{2}$ for the downstream target were removed from the sample. The upstream target events were required to have $R_{\Xi}^{2}<0.90 \mathrm{~cm}^{2}$.

Selection was also based on the beam angles of the $\Xi^{0}$ 's. Because the neutral beam axis had been used in the $\Xi^{0}$ fit, the $\Theta_{x}$ and $\Theta_{y}$ angles calculated from the resulting fit vectors were constrained to fall along the neutral beam. To prevent the selection criteria from being biased by the results of the $\Xi^{0}$ fit, fit-independent quantities which reflected the beam angles of the $\Xi^{0}$ event were substituted for $\Theta_{x}$ and $\Theta_{y}$. First, the momentum vector of the $\Lambda$ from the previous kinematic $\Lambda$ fit was projected from its decay vertex to the z-plane at the face of the lead glass. Then, the momentum-weighted averages, termed $X_{G L}$ and $Y_{G L}$, of the $\mathrm{x}$ and $\mathrm{y}$ positions of the photon showers and the $\Lambda$ at the lead glass were calculated. Due to momentum and energy conservation, these average positions were equivalent to the projected positions of the parent $\Xi^{0}$. As was done with $\Theta_{x}$ and $\Theta_{y}$ for the beam $\Lambda$ sample (See Section 3.3), the $\Xi^{0}$ candidates from each target were summed on a per-run basis in order to establish the average $X_{G L}$ and $Y_{G L}$ for each run. In later passes, histograms of the difference $\left(\Delta X_{G L}\right.$ and $\Delta Y_{G L}$ 


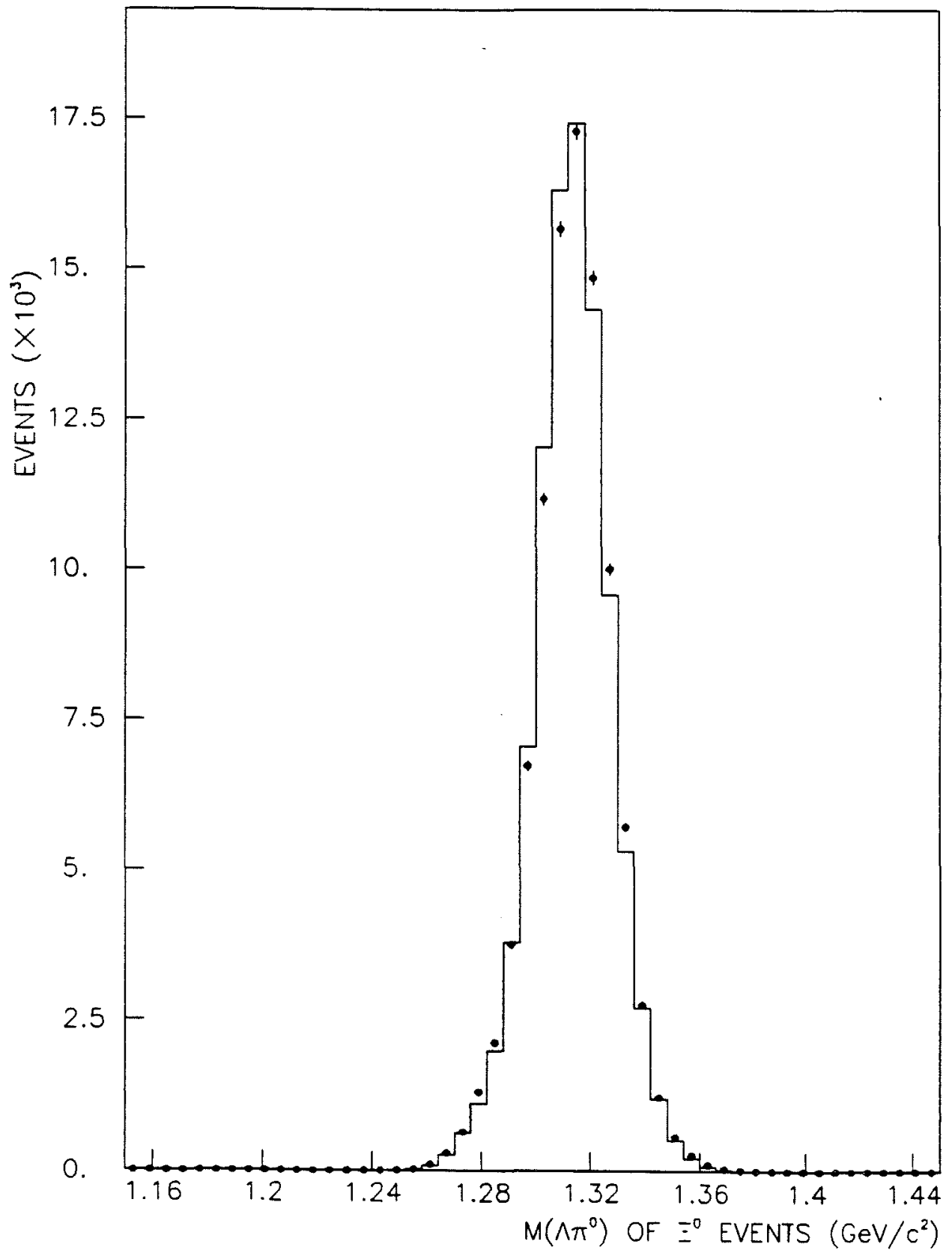

Figure 3.22: $\Xi^{0}$ mass distribution for accepted $\Xi^{0}$ events. (Data - dots , MC - histogram) 


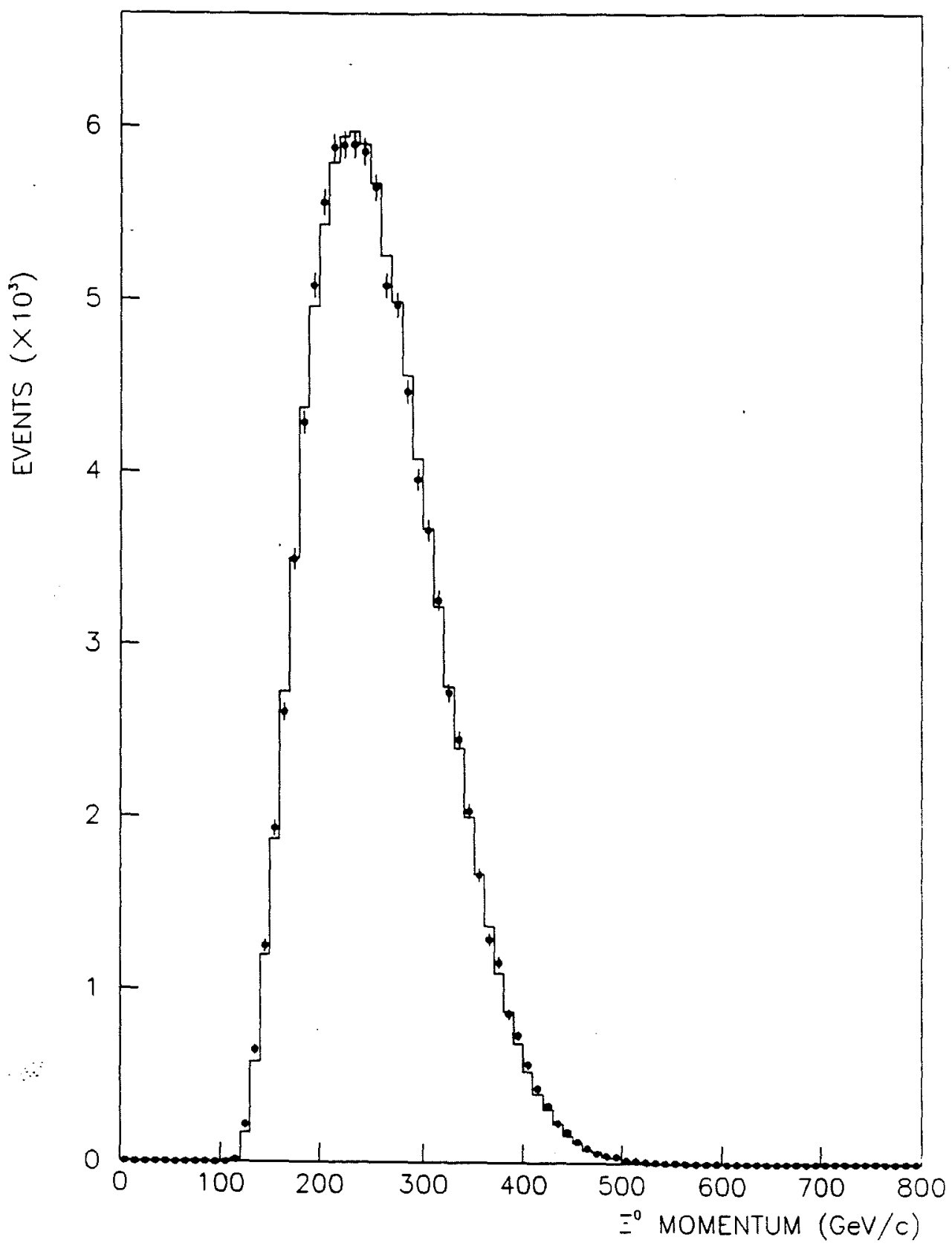

Figure 3.23: $\Xi^{0}$ momentum distribution. (Data - dots , MC - histogram) 

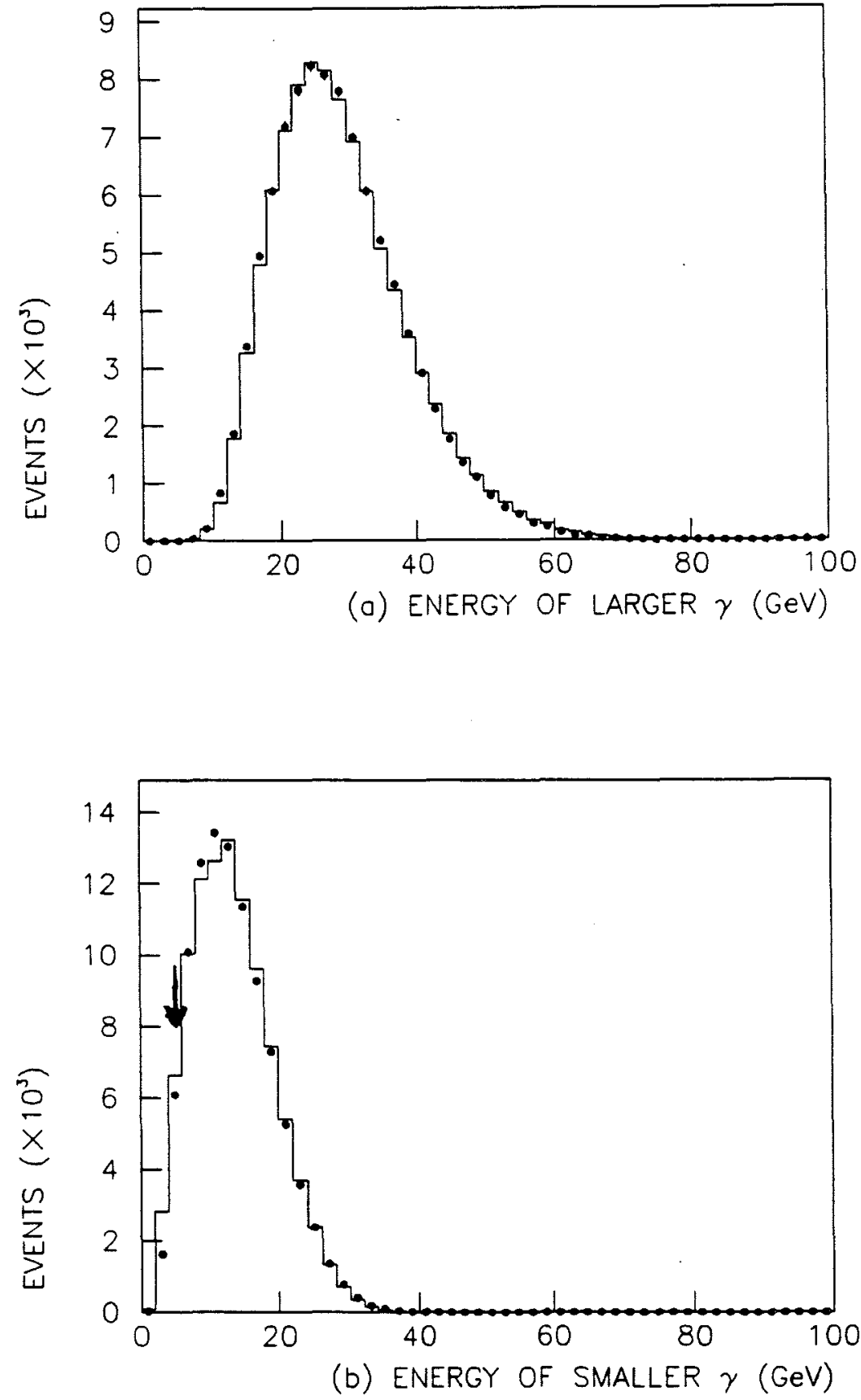

Figure 3.24: Measured energy of photons in $\Xi^{0}$ events. The two photons in an event were separated by energy, the greater being plotted in (a), the lesser in (b). The $5 \mathrm{GeV}$ requirement is indicated by the arrow.(Data - dots , MC - histogram) 


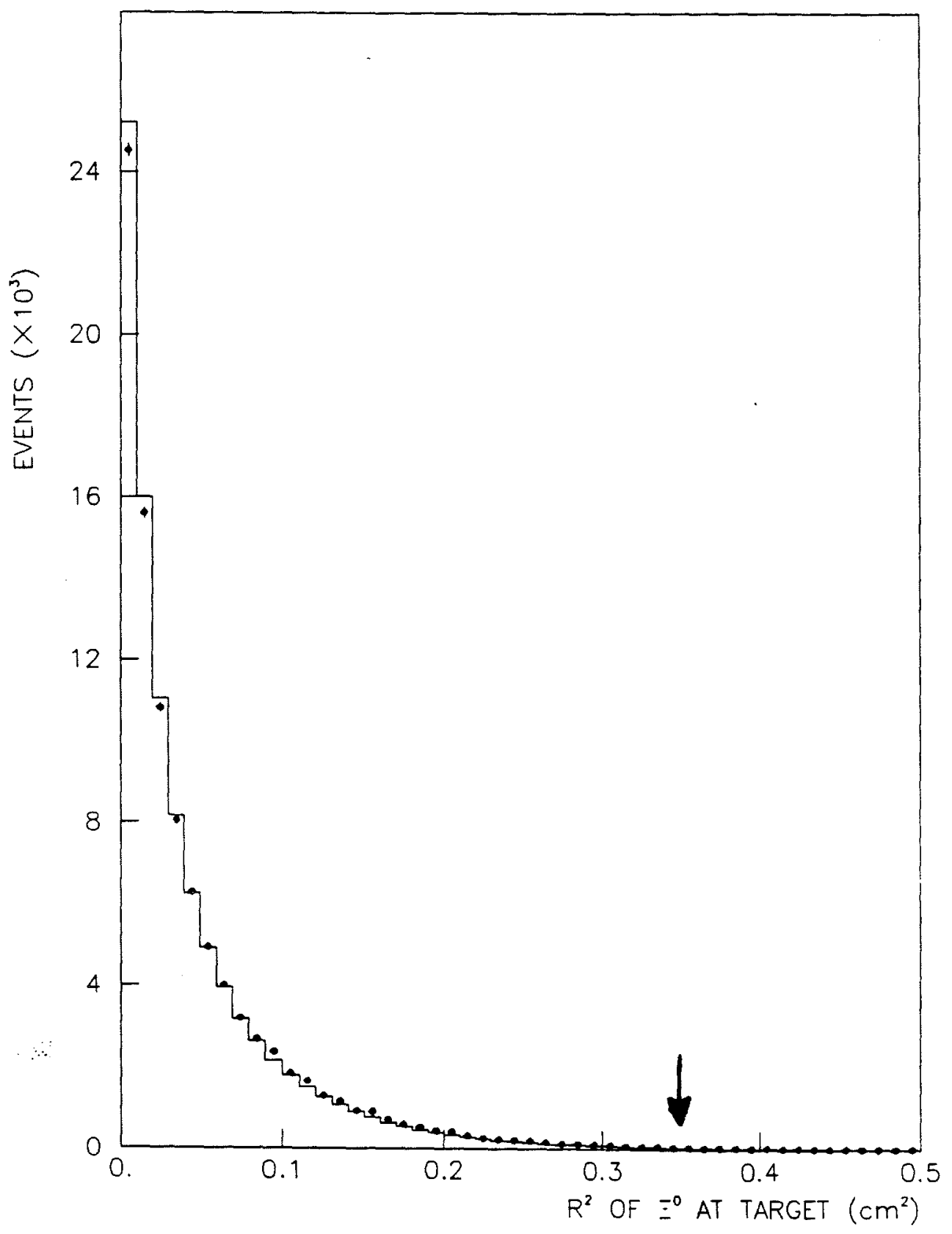

Figure 3.25: $R_{\Xi}^{2}$ for $\Xi^{0}$ events from downstream targets. Only events with $R_{\Xi}^{2}<$ $0.35 \mathrm{~cm}^{2}$ were used.(Data - dots , MC - histogram) 


\begin{tabular}{|c|c|c|c|c|c|}
\hline$\Xi^{0}$ Event & & \multicolumn{5}{|c|}{ Target } \\
\cline { 3 - 6 } Quantity & Units & DE & UE & DW & UW \\
\hline$R_{\Xi}^{2}$ & cm $^{2}$ & $<0.35$ & $<0.90$ & $<0.35$ & $<0.90$ \\
$\left|\Delta X_{G L}\right|$ & $\mathrm{cm}$ & $<4.0$ & $<1.5$ & $<4.5$ & $<1.7$ \\
$\left|\Delta Y_{G L}\right|$ & $\mathrm{cm}$ & $<4.0$ & $<1.5$ & $<4.0$ & $<1.5$ \\
\hline
\end{tabular}

Table 3.5: Final $\Xi^{0}$ target pointing criteria separated by target

) between $X_{G L}$ and $Y_{G L}$ for a $\Xi^{0}$ event and the average for that run were made. The distributions of $\Delta X_{G L}$ and $\Delta Y_{G L}$ are plotted for DE $\Xi^{0}$ events in Figure 3.26. The background events appear in the tails of these distributions. The final $\Xi^{0} R^{2}, \Delta X_{G L}$ and $\Delta Y_{G L}$ criteria separated by target are shown in Table 3.5.

Even after applying the selection criteria discussed above, there remained an apparent contamination from beam $\Lambda$ 's. This was seen in the $R_{\Lambda}^{2}$ distribution for the $\Xi^{0}$ events (Figure 3.27). Studying the $\Xi^{0}$ fit $\chi^{2}$ as a function of this $R_{\Lambda}^{2}$ showed that the large $\chi^{2}$ events typically had low values of $R_{\Lambda}^{2}$. To remove this beam $\Lambda$ background, the $\Xi^{0}$ events were required to have a $R_{\Lambda}^{2}>0.15 \mathrm{~cm}^{2}$.

Additional selection criteria were used to remove the effects of the large proton showers in the lead glass. A substantial fraction of the data $\Xi^{0}$ events also had a large shower in the lead glass due to the interaction of the proton (See Figure 3.28). Such proton-induced showers caused the acceptance of $\Xi^{0}$ events to be systematically biased towards event topologies where the two photon showers did not occur near the position where the proton intersected the lead glass array. This distortion in the acceptance was revealed by comparing the distributions of $\Delta(\gamma-p)$, the distance between the projected proton track at the lead glass and the center of a photon shower. The MC events tended to have more events closer to the proton track than the data events(Figure 3.29). The simplest technique to eliminate this bias would have been to only use data $\Xi^{0}$ events which had a small or zero signal in the block hit by the proton. This criterion was unacceptable because it was caused an added bias towards events where the proton had gone down the crack between two blocks, and thus had a much lower probability 

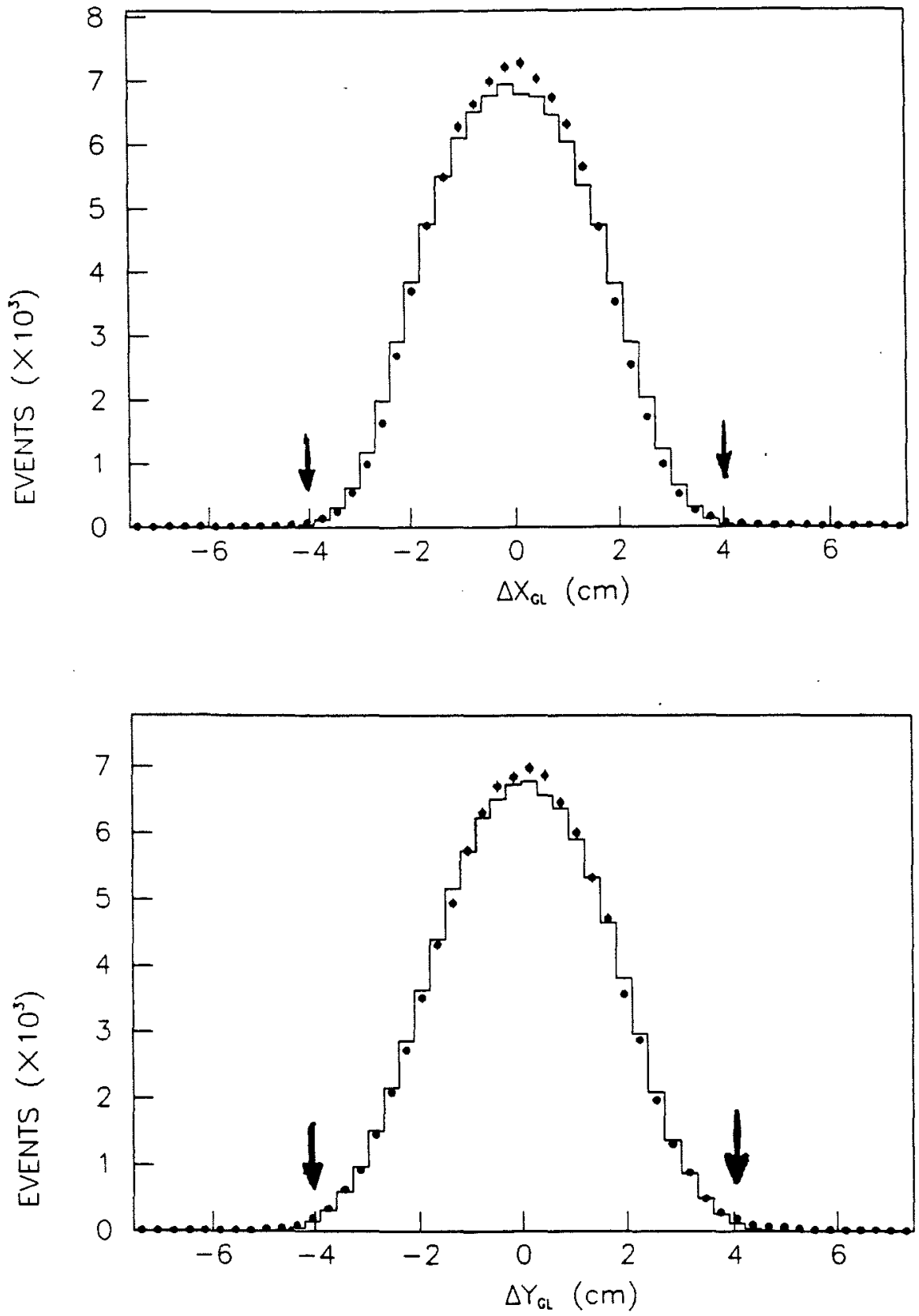

Figure 3.26: $\Delta X_{G L}$ and $\Delta Y_{G L}$ distributions for DE target $\Xi^{0}$ events. The selection criteria used are indicated by the arrows.(Data - dots , MC - histogram) 


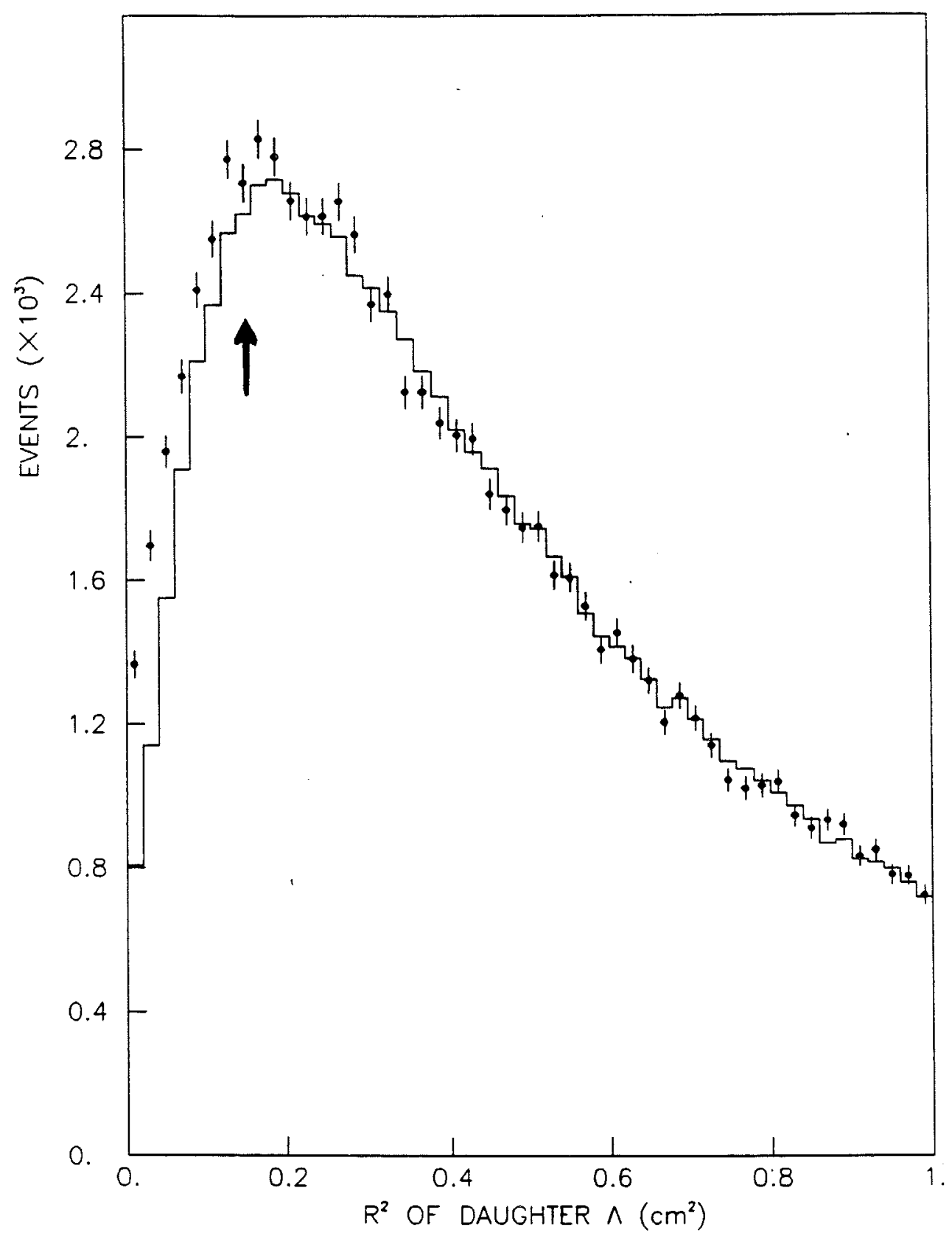

Figure 3.27: $R_{\Lambda}^{2}$ distribution for DS $\Xi^{0}$ events, showing the beam $\Lambda$ contamination at small values of $R_{\Lambda}^{2}$. Only events with $R_{\Lambda}^{2}>0.15 \mathrm{~cm}^{2}$, indicated by the arrow, were used.(Data - dots , MC - histogram) 


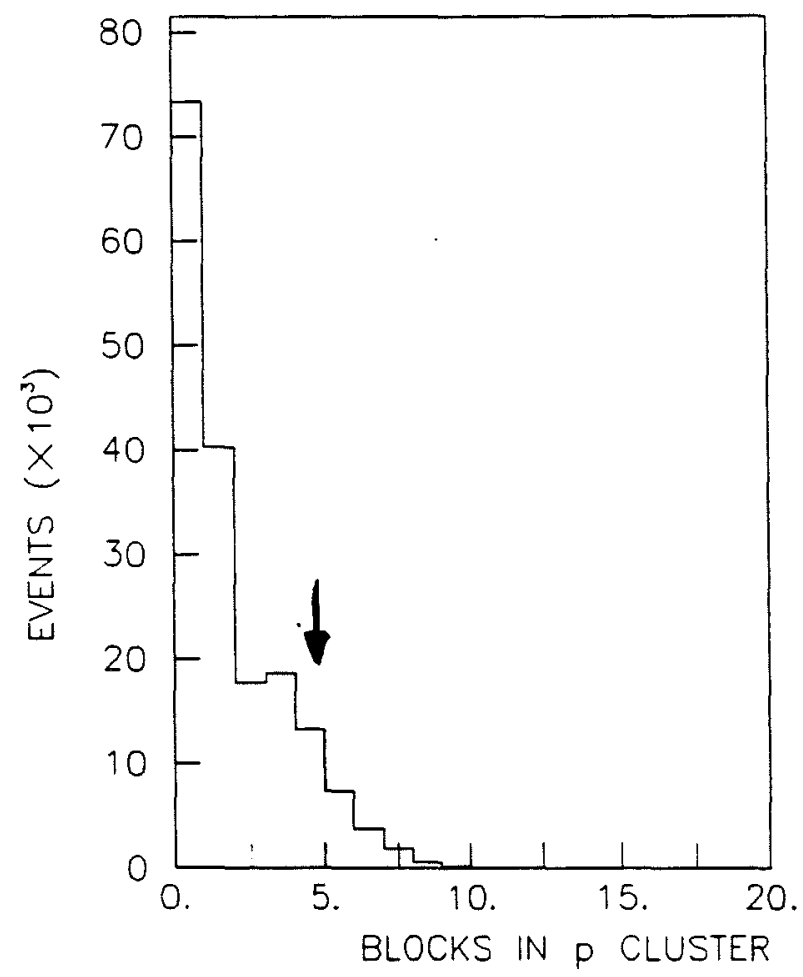

Figure 3.28: Number of blocks in proton cluster in $\Xi^{0}$ events. Most events had 0 blocks, indicating no cluster due to the proton. Only events with fewer then 4 blocks were used in the final $\Xi^{0}$ sample. 


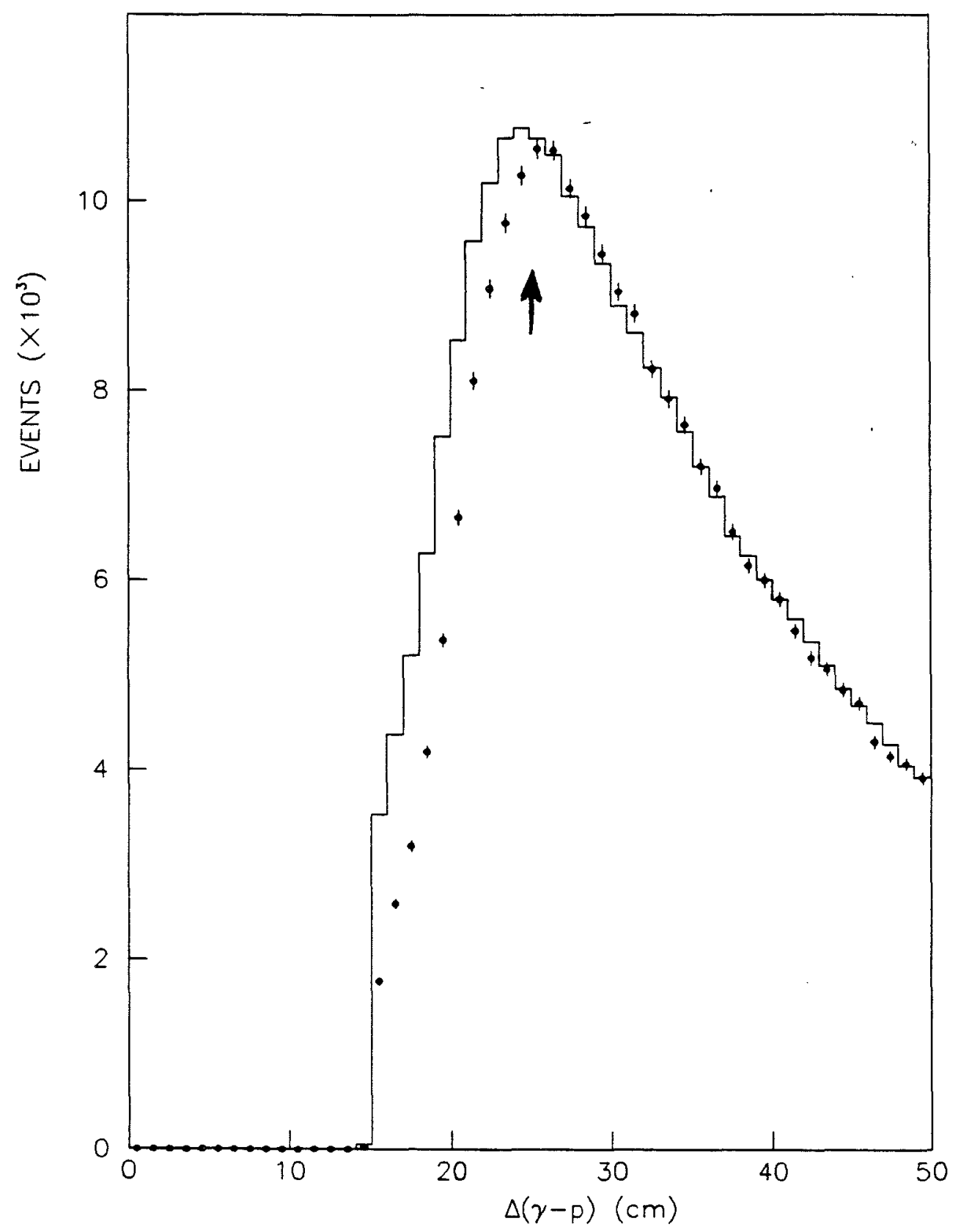

Figure 3.29: Distribution of $\Delta(\gamma-p)$ (distance between photon showers and the proton track) for $\Xi^{0}$ events. The $\Xi^{0}$ event requirement of $\Delta(\gamma-p)>25 \mathrm{~cm}$ is indicated. (Data - dots , MC - histogram) 
of showering. Instead, a cluster in the lead glass array due to the proton was required to have fewer than 4 blocks in it and each photon shower was required to be $>25 \mathrm{~cm}$ from the proton track. The combination of these two requirement avoided the bias due to the cracks and was used in the final $\Xi^{0}$ event selection. 


\section{Chapter 4}

\section{Monte Carlo}

To make the lifetime measurements, it was required to calculated the combined probability that the apparatus would detect a particle decay and that the analysis would reconstruct it successfully. This "acceptance" was not necessarily uniform as a function of particle momentum and decay behavior, and so had to be corrected for. The acceptance was determined using a computer simulation of the experiment with a randomlygenerated sample of decay particles with pre-determined values for the lifetime and the decay asymmetry. For each "Monte Carlo" (MC) event, simulated detector signals were generated. The resulting MC event records were analyzed using the same programs used to reconstruct the real events, so that the MC event samples would reflect any biases due to the reconstruction and selection criteria.

The MC program generated candidate $\Xi^{0}$ and $\Lambda$ decay events which were randomly distributed according to chosen values for the lifetime and decay asymmetry. These events were generated with momentum and neutral beam phase space distributions to match those found in the real events. The program simulated the effects of the apertures of the collimator and apparatus on these MC events. It simulated the effects of multiple scattering and the magnetic fields on the charged particle trajectories, For MC events which satisfied the simulated triggers, event records were generated simulating the 
response of each of the charged particle and photon detector elements. In most cases, the inputs to the detector simulations were derived from detailed analyses of the signals seen from the real events.

\subsection{Neutral Beam Simulation}

The neutral beam part of the MC provided $\Lambda$ and $\Xi^{0}$ events which had the same distributions in beam phase $\operatorname{space}\left(P, \Theta_{x}, \Theta_{y}\right)$ and origin at the $\operatorname{target}\left(X_{T}, Y_{T}, Z_{T}\right)$ as were seen in the real data. Possible particle origins and trajectories were generated at the target, and then checked against the physical apertures of the collimator. The particle momentum $(P)$ was chosen randomly from a input spectrum that was adjusted so that the reconstructed MC momentum distribution would match that seen in the data.

First, a point $\left(X_{T}, Y_{T}\right)$ was randomly chosen on the target according to Gaussian distributions whose centers and widths simulated the shape of the incident proton beam at the target. To simulate an observed deviation of the proton beam from a pure Gaussian distribution, the target was tilted slightly ( 5 mrad.) to the z-axis of the neutral beam. Points that did not fall within the cross-section of the target were rejected. $Z_{T}$ was chosen randomly along the length of the physical target. Next, a point was chosen at random distributed uniformly across the aperture (radius $=0.168 \mathrm{~cm}$ ) of the collimator at the beginning of the defining section $(z=-335.4 \mathrm{~cm})$. The points at the target and collimator served to define $\Theta_{x}$ and $\Theta_{y}$. The particle trajectory was then projected to the end of the defining section and to the exit of the collimator, and checked that it passed through the apertures of the collimator at these points.

The beam Gaussian centers and widths used were adjusted slightly on a run- byrun basis to match the fluctuations in the data. The position distributions of the reconstructed decay, when projected back to the target, were dominated by resolution effects. It was more accurate to determine the beam Gaussians by correlations with 
the projected distributions at the face of the lead glass array. Because the experiment coordinate system had not been externally defined, the surveyed positions of the targets and collimator were difficult to use. Instead, the targets and collimator positions were established by looking at the averages of the data distributions combined for all the runs between changes in the chamber centers.

Previous experiments [18][21] had found that the production of $\Lambda$ 's and $\Xi^{0}$ 's is a function of the transverse momentum $P_{T}$ of the particle relative to the axis of the incident proton beam. In this experiment, the proton beams had an incident angle which was at most $1 \mathrm{mrad}$, and so $P_{T}$-dependent effects were expected to be minimal. As the $\mathrm{MC}$ events simulated the $\Theta_{x}$ and $\Theta_{y}$ distributions of real beam $\Lambda$ events as a function on momentum sufficiently, no $\boldsymbol{P}_{T}$-dependence was introduced into the MC beam phase space.

\subsection{Particle Decay Simulation}

For candidate MC events which passed the collimator cuts, the MC program simulated the decay of the beam particle into the daughter particles seen by our detectors. The $z$ of the decay vertex along the particle' trajectory from the target was randomly chosen from an exponential distribution, $\exp (-m z / p c r)$, where $m$ was the known rest mass of the decaying particle, $p$ its simulated momentum and $\tau$ the lifetime chosen for the MC. In the case of the $\Lambda$ 's, a faster procedure was used which weighted the choice of the particle momentum by the relative integrated probability that a particle of a particular momentum would decay in the decay volume between the V1 and DK trigger counters. A decay point was chosen and then the momenta were Lorentz transformed to the $\Lambda$ 's rest frame, where an isotropic decay into $p$ and $\pi^{-}$was performed, with the resulting momenta Lorentz transformed back to the lab frame.

The $\Xi^{0}$ 's were more complicated. The same technique of decay-volume weighting of the momentum spectrum was used, but the range of allowed $\Xi^{0}$ decay vertices was 
from the end of the defining collimator to the DK counter. The $\Xi^{0}$ was then Lorentz transformed to its rest frame, where it was decayed isotropically into the $\Lambda$ and $\pi^{0}$. The momenta for these daughter particles were then transformed back to the lab frame. The decay of the $\pi^{0}$ into its two photons was then simulated. If the decay point of the $\Xi^{0}$ was upstream of the exit of the collimator, then the trajectories of the $\Lambda$ and photons were checked at the collimator apertures, and an event rejected if they did not pass through them. Next the decay vertex of the $\Lambda$ was randomly determined from the $\Xi^{0}$ decay point using the $\Lambda$ lifetime. If the $\Lambda$ decay vertex fell between the V1 and DK counters, the event was accepted.

For MC $\Xi^{0}$ events with an accepted $\Lambda$ decay vertex, the decay of the $\Lambda$ into its daughter particles was then simulated. It was here that the decay asymmetry of the $\Xi^{0}$ was taken into account. The $\Xi^{0}$ beam polarization was measured (See Section 5.4.3), and found to be consistent with zero. The MC $\Xi^{0}$ events were thus generated without any beam polarization. In the case of no $\Xi^{0}$ polarization, the daughter protons have an angular distribution of

$$
\frac{d n}{d \cos \theta}=\frac{n}{2}\left(1+\alpha_{\Lambda} \alpha_{\Xi^{0}} \cos \theta\right)
$$

where $\theta$ is the angle between the outgoing proton and the $\Lambda$ momentum vector in the rest frame of the $\Lambda$ reached by first Lorentz transforming to the $\Xi^{0}$ rest frame. The existing world average values for $\alpha_{\Lambda}$ and $\alpha_{\Xi^{0}}$ of 0.628 and -0.458 were used in generating the MC events. The protons were randomly distributed according to this equation, with the $\pi^{-}$'s having the opposite direction in the rest frame. The daughter momenta were then Lorentz transformed back to the lab frame. As is shown in Figure 4.1, the distribution of reconstructed $\Xi^{0}$ events did not exhibit the linear dependence on $\alpha_{\Lambda} \alpha_{\Xi}$ expected from Equation 4.1. This was because the acceptance was not uniform as a function of $\cos \theta$. 


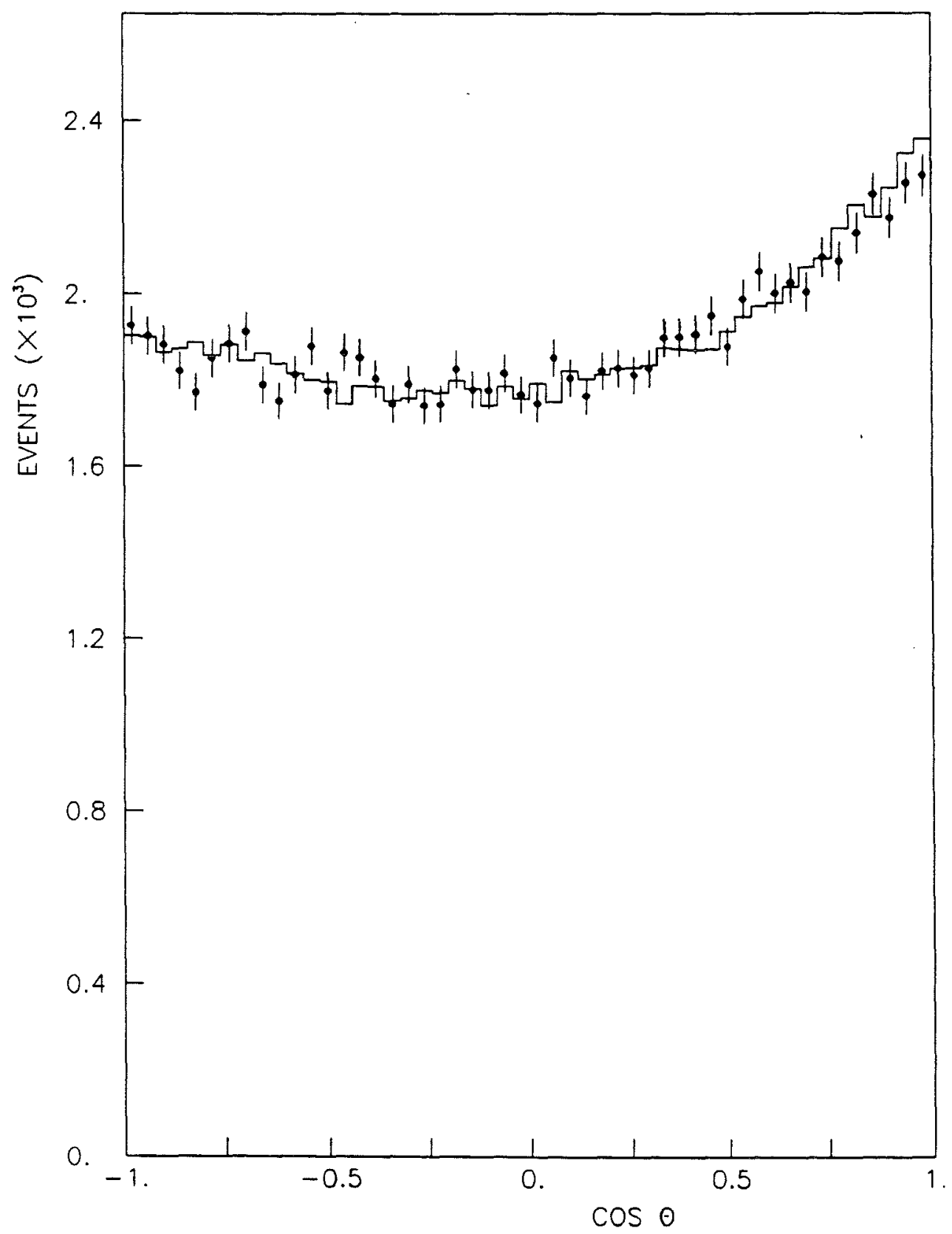

Figure 4.1: Distribution of $\Xi^{0}$ events as a function of $\cos \theta$. Note that the expected linear dependence on $\alpha_{\Lambda} \alpha_{\Xi}$ is masked by the variation in the acceptance. (Data-dots, MC - histogram) 


\subsection{Charged Particle Simulation}

Modelling the charged particle trajectories with the MC consisted of determining their path through the apparatus, including the bend due to the analyzing magnets field, checking whether they passed through all apertures, and allowing for multiple scattering and decay of the $\pi^{-}$'s.

\subsubsection{Multiple Coulomb Scattering}

Any charged particle passing through matter undergoes small-angle elastic scatterings which are predominantly due to Coulombic interactions with nuclei. This multiple scattering has the effect of reducing the position resolution on the tracks of the particles. This scattering was modelled in the MC by having each distinct layer of apparatus material scatter the charged particles through a random angle according to a Gaussian approximation[22] of width (in radians)

$$
\theta_{0}=\frac{14.1 M e V / c}{p \beta} Z_{\text {inc }} \sqrt{L / L_{R}}\left(1+\frac{1}{9} \log L / L_{R}\right)
$$

$p, \beta$ and $Z_{\text {inc }}$ are the momentum(in $M e V / c$ ), velocity and charge number of the particle ( 1 for protons and $\pi^{-}$'s) and $L / L_{R}$ was the thickness of the material in terms of radiation lengths. Our apparatus had been designed to minimize the amount of material which could cause this scattering. The spectrometer material from the DK counter to chamber C6 totalled $9.5 \%$ of a radiation length. The effect of this scattering on the position resolution was weighted by how far upstream it occurred. Nearly half $(4.3 \%$ out of $9.5 \%$ ) of the total radiation lengths occurred in the A and B hodoscope elements, which only affected the tracks in C6, a chamber which the low momentum particles most affected by multiple scattering often missed. That this model gave the correct amount of multiple scattering was checked by looking at the C4 y-residual as a function of the particle momentum. Multiple scattering would impart a linear dependence of the width of the residual as a function of $1 / p^{2}$. In $\Lambda$ 's, this was hard to evaluate, as the asymmetric 
106

decay yielded very soft pions or very stiff protons. $K_{\pi 2}$ decays, however, showed this effect clearly and this multiple scattering model gave good agreement between the MC and data residual widths.

\subsubsection{Magnetic Fields}

The $B_{Y}$ fields of the analysis magnets were modelled as two separate uniform dipole fields each having a length equal to that assumed in the reconstruction (See Section 3.2.3). The particle trajectories were bent in a continuous arc while they passed through the field of each magnet, being allowed to drift in the gap between the two fields. The magnitude of the fields was calculated from the parameters used in the reconstruction (Table 3.3). Particles whose path hit the walls of the magnets, or failed to pass through the apertures of the mirror plates were rejected.

The effect of each fringe field was simulated as an instantaneous rotation of the $P_{x y}$ momenta vector about the $z$-axis at each end of the two magnets. The strength of the fringe fields was calculated from the field map data by integrating $B_{Z} d \ell$ in the region of the edge of the $B_{Y}$ field for different heights (y-positions) in the aperture of the magnet. These were converted to equivalent $P_{T}$ values and then fit to $P_{T}(y)=m y+b$ where $P_{T}$ was near zero at $y=0$.

\subsubsection{Pion decay}

The lifetime of a charged pion is $2.603 \times 10^{-8} \mathrm{~s}$. Its major decay mode is into a neutrinomuon $(\nu-\mu)$ pair. If this occurred, it would bend the apparent trajectory of the $\pi^{-}$, affecting the position resolution. For a typical $(25 \mathrm{GeV}) \pi^{-}$in our experiment, the cumulative probability that it would decay in flight before chamber C6 was $3 \%$. We compared the MC distributions with and without the simulation of these decays, and concluded that they produced no observable effects. 


\subsection{MWPC's and Scintillation Counters}

As the trajectories of the proton and $\pi^{-}$through the apparatus were calculated, the MC simulated the signals created by them in the MWPCs and in the scintillation counters of the trigger. For each chamber plane, the MC determined the wire closest to the simulated particle trajectory. The number of this wire was entered in an array of "hit" wires, which was later put onto the MC event records. These chamber hits were corrected for the chamber inefficiencies and had extra wires added to reflect the multiple hits due to double-hits and delta-rays. Extra chamber hits due to random noise were also included. For the counters, the MC program determined which elements the particle passed through and corrected the simulated outputs for any inefficiencies. The amount of inefficiency and multiple hits required was determined by analyzing the actual experimental data, and then requiring the MC events to reproduce the measured results.

\subsubsection{Chamber Inefficiencies}

As mentioned in Section 2.9.1, the real event reconstruction program calculated the apparent inefficiency of each chamber plane. The wires were grouped by MWPC amplifier card (four wires per card), and statistics on inefficiency kept for each card in each chamber plane for each run in the experiment.

For generating MC events, these card inefficiencies were averaged over all the runs to minimize the effects of statistical fluctuations. The run-to-run variation in the inefficiencies was simulated by multiplying these averaged inefficiencies by a correction factor, one per plane, which was different for each run. For every hit wire, the card on which it resided was determined and a random number between zero and one was chosen. If this random number was less than the inefficiency fraction, then the hit was removed from the list. These correction factors not only accounted for the run-to-run changes, but also raised the inefficiencies to correct for the bias in the reconstructed 


\begin{tabular}{|c|c|c|c|c|c|c|}
\hline \multirow{2}{*}{ Plane } & \multicolumn{6}{|c|}{ Chamber No. } \\
\cline { 2 - 7 } & 1 & 2 & 3 & 4 & 5 & 6 \\
\hline Y(or V) & 2.0 & 2.2 & 3.4 & 1.6 & 4.4 & 4.3 \\
X (or U) & 9.2 & 6.3 & 2.9 & 6.5 & 1.9 & 6.0 \\
\hline
\end{tabular}

Table 4.1: Average apparent 2-hit frequency (\%) in reconstructed events

data towards events without missing hits. These factors were adjusted until the reconstructed MC events yielded the same apparent efficiencies as that found in the data. These inefficiencies raised the geometric $\chi^{2}$ of the reconstructed MC events slightly, but did not have a large effect otherwise.

\subsubsection{Multiple hits and chamber noise}

When a charged particle passed between two adjacent MWPC wires, there was a chance that both wires would have large enough signals induced in them to be recorded as hits in the detector. This probability was greatest when the particle passed close the midpoint between the wires. These "double-hits" were used to advantage by the event reconstruction to improve the position resolution. Higher percentages of tracks which created such double-hits in a plane were reflected in smaller widths of the residual distribution. Such double-hits can also be due to low-energy electrons (delta-rays) created by the charged particles interacting with matter. The MC simulated the doublehits based on their observed frequency in the reconstructed real events.

The raw chamber hits for the reconstructed events were examined by the analysis program. It determined for each plane which hits were associated with the tracks (on-track) and which were not (off-track). The on-track hits were broken down into clusters of adjacent wires, and how many 1-wire,2-wire, 3-wire, etc. clusters there were was tabulated. The resulting percentages of 2-wire on-track clusters per plane averaged over the experiment are shown in Table 4.1.

At first, the MC program assumed all the 2-wire on-track clusters were due to true double-hits. The fraction of the space around the mid-point between wires which 


\begin{tabular}{|c|c|c|c|c|c|c|}
\hline Plane & \multicolumn{6}{|c|}{ Chamber No. } \\
\cline { 2 - 7 } & 1 & 2 & 3 & 4 & 5 & 6 \\
\hline $\mathrm{Y}$ (OR V) & 8.4 & 11.9 & 8.6 & 10.2 & 10.5 & 15.3 \\
$\mathrm{X}$ (OR U) & 9.2 & 6.3 & 2.9 & 6.5 & 1.9 & 6.0 \\
\hline
\end{tabular}

Table 4.2: Average probability(\%) of noise clusters per MWPC plane

resulted in a simulated double-hit was adjusted for each plane on a per-run basis, so that the reconstructed $\mathrm{MC}$ events had the same percentage of 2-wire clusters on their tracks as was seen in the data. This wire separation fraction was typically, especially for chambers $\mathrm{C} 1,2$ and 3, larger then the observed 2-wire hit probability because the reconstruction had a strong bias favoring events with such double-hits. While this MC procedure gave the correct 2-wire percentages, the MC events still had residual widths and average geometric fit $\chi^{2}$ 's which were smaller than the data. This indicated that the double-hit percentages being used were too great. The surplus 2-wire clusters in the data were ascribed to random processes such as the delta-rays and cross-talk between wires. These processes were simulated by generating $1.6 \%$ of the chamber track hits in each plane with random hits in adjacent wires. With this fraction of 2-wire clusters removed from the percentages of double-hits used in the MC, the residual widths and $\chi^{2}$ 's agreed with those in the data. This improved the agreement between the distribution of $\mathrm{MC}$ and real events as a function of the separation between the reconstructed proton and $\pi^{-}$tracks at the first chamber (Figure 4.2). Events with a track separation at $\mathrm{C} 1$ of $<0.5 \mathrm{~cm}$ in the $\mathrm{x}$-view or $<0.25 \mathrm{~cm}$ in the $\mathrm{y}$-view were cut from the final event samples, so that the lifetime fits would not be sensitive to the exact double-hit percentages and their effects on the resolution,

The off-track hits found in the data were ascribed to random tracks and noise. Statistics for these off-tracks clusters were tabulated both for how many such noise clusters there were per plane in an event, and for how many wires made up each noise cluster. These statistics were tabulated for each run of the experiment. The probability of a noise cluster in each plane averaged over the experiment is shown in Table 4.2. The 

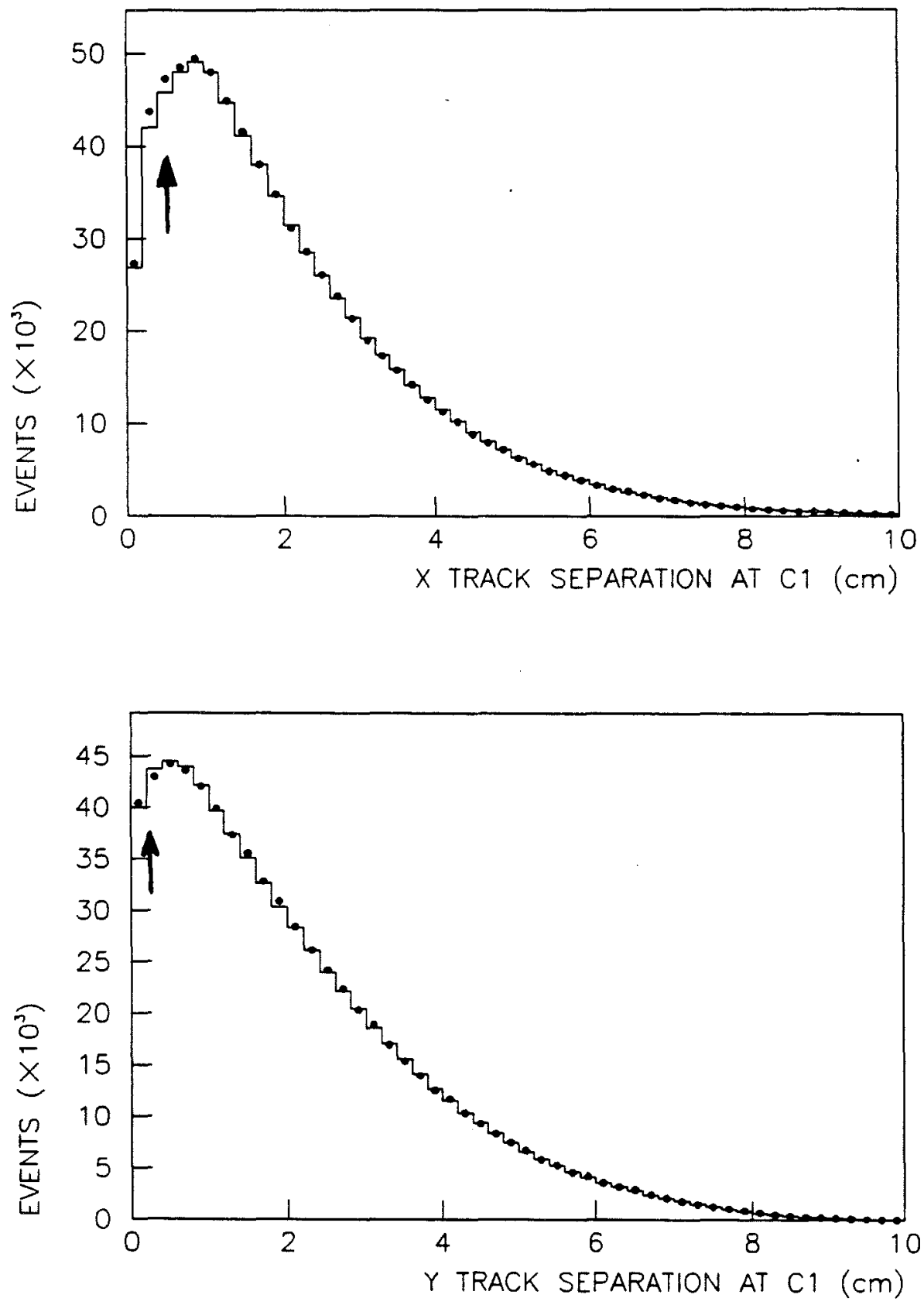

Figure 4.2: Separation (cm) in $\mathrm{X}$ and $\mathrm{Y}$ of $p$ and $\pi^{-}$tracks at chamber $\mathrm{C} 1$ for $\Lambda$ events. (Data - dots, MC - histogram) 
MC program was tried both with and without these random noise hits being simulated. Other than a slight decrease in the percentage of reconstructed events, these noise clusters had no significant effect on the results of the MC, and were thus not included.

\subsubsection{Trigger counter efficiencies}

As mentioned in Section 2.9.2, the F.C5 trigger events were analyzed to determine the efficiency of the scintillation counters in the $\Lambda$ and $\Xi^{0}$ triggers in registering the passage of charged particles. A fiducial cut requiring that the track be greater than $1 \mathrm{~cm}$ in from the edges of the counter was imposed to make the efficiency results insensitive to the position resolution. These counter efficiencies were simulated in the generated MC events. Figure 4.3 compares the distribution of $\pi^{-}$tracks at the $B$ hodoscope from real and MC $\Lambda$ events from the $\Lambda$ trigger.

The efficiencies of the proton and $\pi$-killer counters were also calculated from the F.C5 trigger events. The proton counters appeared to have a consistent $93 \%$ efficiency, but MC studies comparing F.C5 and $\Lambda$ trigger event distributions indicated that the proton counter response was more consistent with near $100 \%$ efficiency. This discrepancy could be caused by a slight mis-timing of the proton counter signals into the latch gate used to determine the efficiency. The distribution in $\mathrm{x}$ of proton tracks from $\Lambda$ trigger events at the plane of the proton counters is shown in Figure 4.4. The MC generated latch bit signals for all the counters which had been fired in an event.

Selection criteria were added to the event analysis to both ensure that the real events had satisfied the $\Lambda$ trigger with their proton and pion tracks and to minimize any effect from small mis-matches of the outside counter edges between the MC and the real apparatus. At the $\mathrm{B}$ hodoscope, the $\mathrm{x}$ position of the $\pi^{-}$track was required to be $>-94 \mathrm{~cm}$ and $<90 \mathrm{~cm}$, as indicated in Figure 4.3. At the A hodoscope, the cuts were $>-64 \mathrm{~cm}$ and $<58 \mathrm{~cm}$. At the proton counters, the $x$ position of the proton track was required to be $>7 \mathrm{~cm}$ or $<-14 \mathrm{~cm}$, as indicated on Figure 4.4 . 

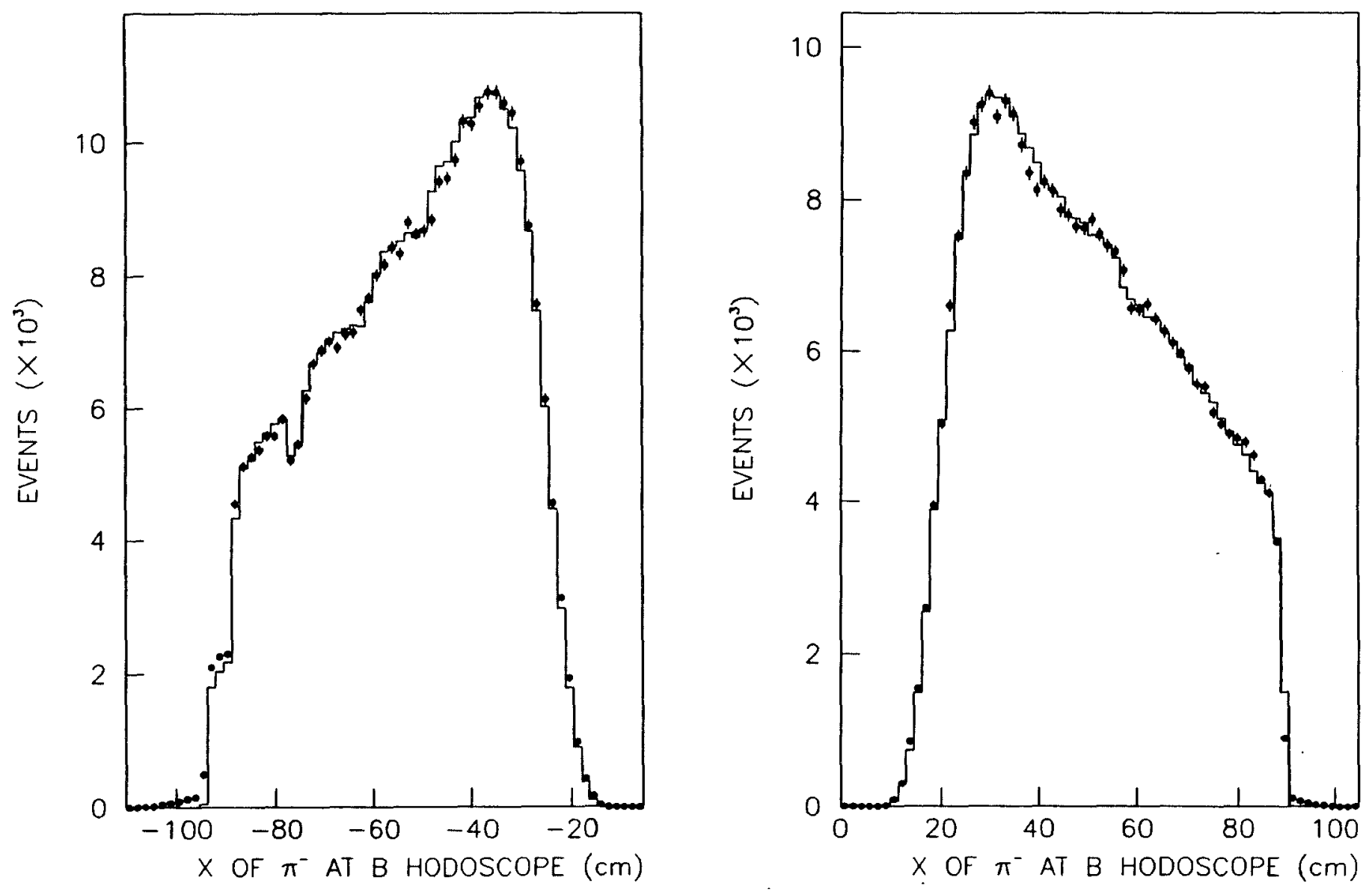

芯

Figure 4.3: Distribution in $\mathrm{X}$ of $\pi^{-}$'s from $\Lambda$ trigger events projected to the plane of the B hodoscope. (Data - dots, MC - histogram) 

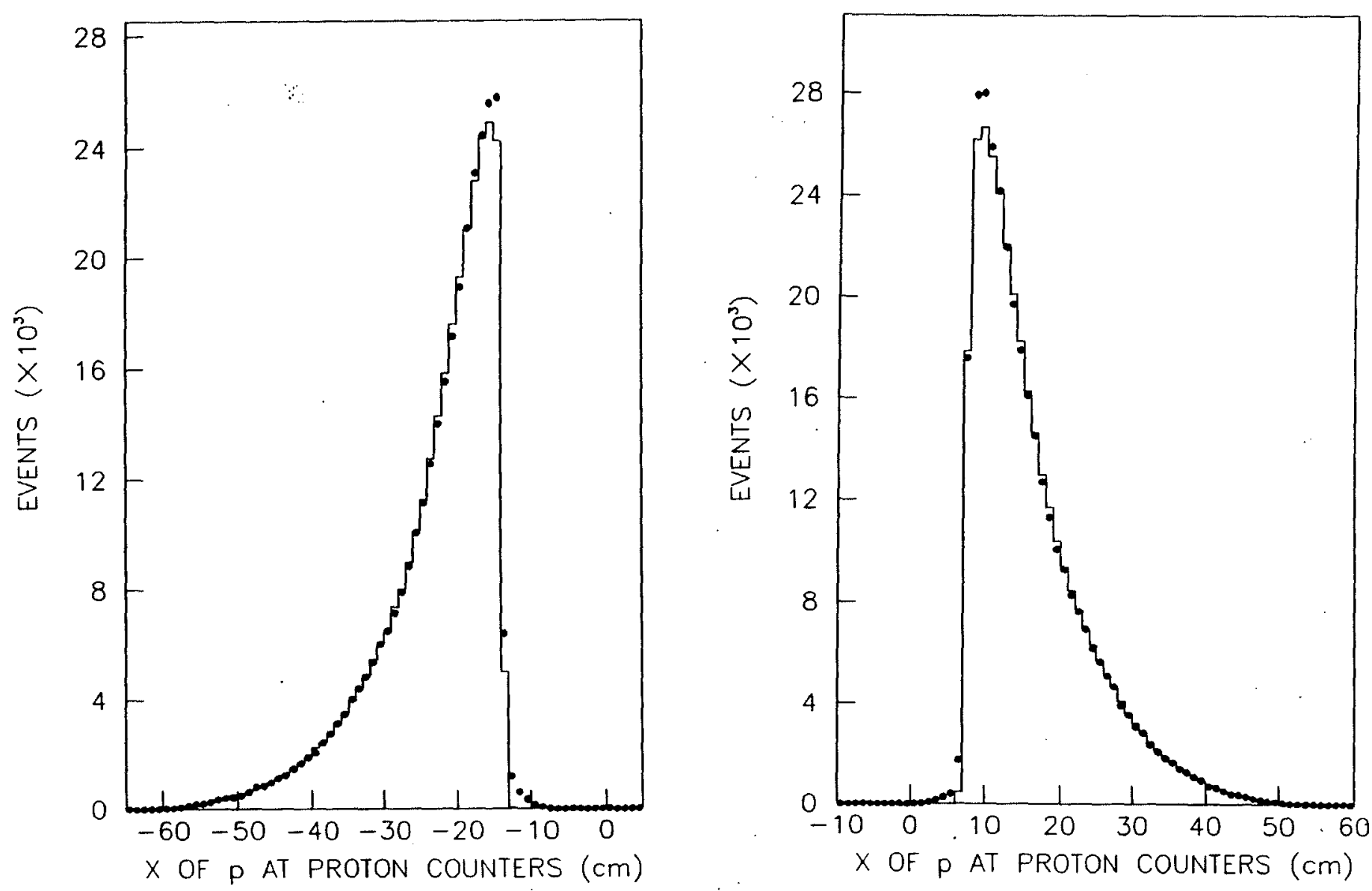

Figure 4.4: Distribution in $\mathrm{X}$ of protons from $\Lambda$ trigger events projected to the plane of the proton counters. (Data - dots, MC - histogram) 


\subsubsection{F.C5 and $\Lambda$ Trigger Simulation}

For each candidate event, the MC program checked if it satisfied the given trigger. The F.C5 trigger was simulated by checking that there was at least one wire hit in the $y$-plane of Chamber C5. The $\Lambda$ trigger was simulated by examining the latch bits of the trigger counters and requiring that the trigger logic described in Section 2.5.3 was satisfied.

\subsection{Photons}

The MC program projected the simulated photons from the $\Xi^{0}$ decay to the plane of the lead glass array. If either of these trajectories intercepted the walls of the magnets, the event was rejected. Events were also rejected if either photon failed to hit a block in the array. The MC simulated the shower caused in the array by each photon and determined the resulting ADC and TDC signals.

The overall energy response of the array to the incident photons was simulated using E/P distributions derived from the $e^{+} e^{-}$calibration data. Histograms of the $\mathrm{E} / \mathrm{P}$ distributions were prepared with the $e^{+} e^{-}$data from non-hole,non-edge tracks broken down by the track momentum $P$ into $4 \mathrm{GeV}$ bins. These distributions reflected both the resolution and any non-linearity of the response. The $e^{+} e^{-}$data was further divided into two samples, one with tracks which which landed near the center of a block and those with positions away from the center. The events away from center had greater tails in their response towards low $\mathrm{E} / \mathrm{P}$, indicating losses due to shower energy disappearing down the cracks between blocks. The MC simulation used the energy and position of the photon to choose the appropriate E/P histogram, then randomly selected an $\mathrm{E} / \mathrm{P}$ value according to the distribution. This value was multiplied by the energy of the photon to determine the amount seen by the array.

The lateral spread of the energy of the shower in $\mathrm{x}$ and $\mathrm{y}$ was simulated by the sum 
of two Gaussian distributions. The first Gaussian, representing the core of the shower, had a $\sigma$ of $0.47 \mathrm{~cm}$ in both $\mathrm{x}$ and $\mathrm{y}$, while the second, representing the tails, had a $\sigma$ of $2.33 \mathrm{~cm}$. The fraction of the shower energy in the core Gaussian was randomized as a Gaussian distribution centered at 0.78 with a $\sigma$ of 0.08 . Slightly narrower showers with sigmas of $0.21 \mathrm{~cm}$ and $1.94 \mathrm{~cm}$, respectively, were used to simulate the lateral spread for runs without the pre-converter. Each Gaussian was centered at the position of the photon at the array. The fraction of each distribution in a particular block was determined by calculating the area under the $x$ Gaussian which fell between the limits in $\mathbf{x}$ of that block, and multiplying it by the area under the $y$ Gaussian within the block. This was done for the block the photon hit as well as the six nearest-neighbor blocks. The fractions in each block were then normalized so that their sum was unity. The portion of the shower energy associated with the distribution was multiplied by the fraction to yield the amount in each block. If the center block was adjacent to the hole or to the outer edge of the array, then the amount of the distribution over the hole or off the edge was calculated and included in the sum, with the resulting portion of the shower energy assumed lost.

Once the amount of energy in each block due to both photons was determined, then the ADC and TDC signals were simulated. The ADC signal in each block was calculated by dividing the energy by the gain constant for that block. The response of the TDC discriminators as a function of ADC counts was parameterized by an error function, which was then used to randomly determine if the regular TDC had a signal for a given block in each event. The trigger TDC response curve had a similar shape, but with a $50 \%$ efficiency point at about 80 ADC counts.

\section{6 $\Xi^{0}$ Trigger Simulation}

The trigger TDC signals, along with any $\pi$-killer signals due to the charged particle were used to simulate the $\gamma$ trigger. The program implemented the same cluster-counting 
logic as in the hardware trigger, and removed blocks from the trigger as required by examining the latch bits of the $\pi$-killer counters. Cuts were added to the event analysis to ensure that the real data events had also triggered the $\gamma$ trigger with their photon showers, rather than due to insufficiently vetoed hadron showers. The cuts required that both photons showers had in-time trigger TDC signals, and that were not vetoed by $\pi$-killer overlaps.

The $\Xi^{0}$ trigger also required no signal from any of the V2 counters. The proton and $\pi^{-}$tracks had been projected to the plane of these counters, and the event was rejected if they intersected any of the six counters.

An unexpected depletion of real $\Xi^{0}$ events was observed as a function of the $\mathbf{x}$ position of the $\pi^{-}$at the $B$ hodoscope, peaking at $x=+70 \mathrm{~cm}$ (Figure 4.5). The explanation was provided by examining events from the $\Lambda$ trigger for V2 latch signals which were not due to the tracks in the event. This revealed that most of the time, the $V_{O R}$ of the six V2 counters fired for only $20 \%$ of the events, but that when the $\pi^{-}$ was near $+70 \mathrm{~cm}$, this veto rate rose to near $60 \%$ (Figure 4.6 ). This increase in the veto rate was apparently due to the low momentum $\pi^{-}$'s striking the wall on the West side of the pit the apparatus was located in, creating a shower of secondary particles which, if the angle was right, would hit the V2 counters on the left side of the array. This was confirmed in the $\Lambda$ data by an increase in the occurrence of hits in the veto counter on that side of the lead glass array, while the frequency of hits in the far-side counters remained constant. Rather than cut out this whole region of the B hodoscope $\mathrm{x}$ distribution, it was decided to make corrections in the MC $\Xi^{0}$ trigger simulation. MC events were randomly vetoed based on the probabilities, as a function of the $\pi^{-}$ position, measured from the $\Lambda$ trigger data. 


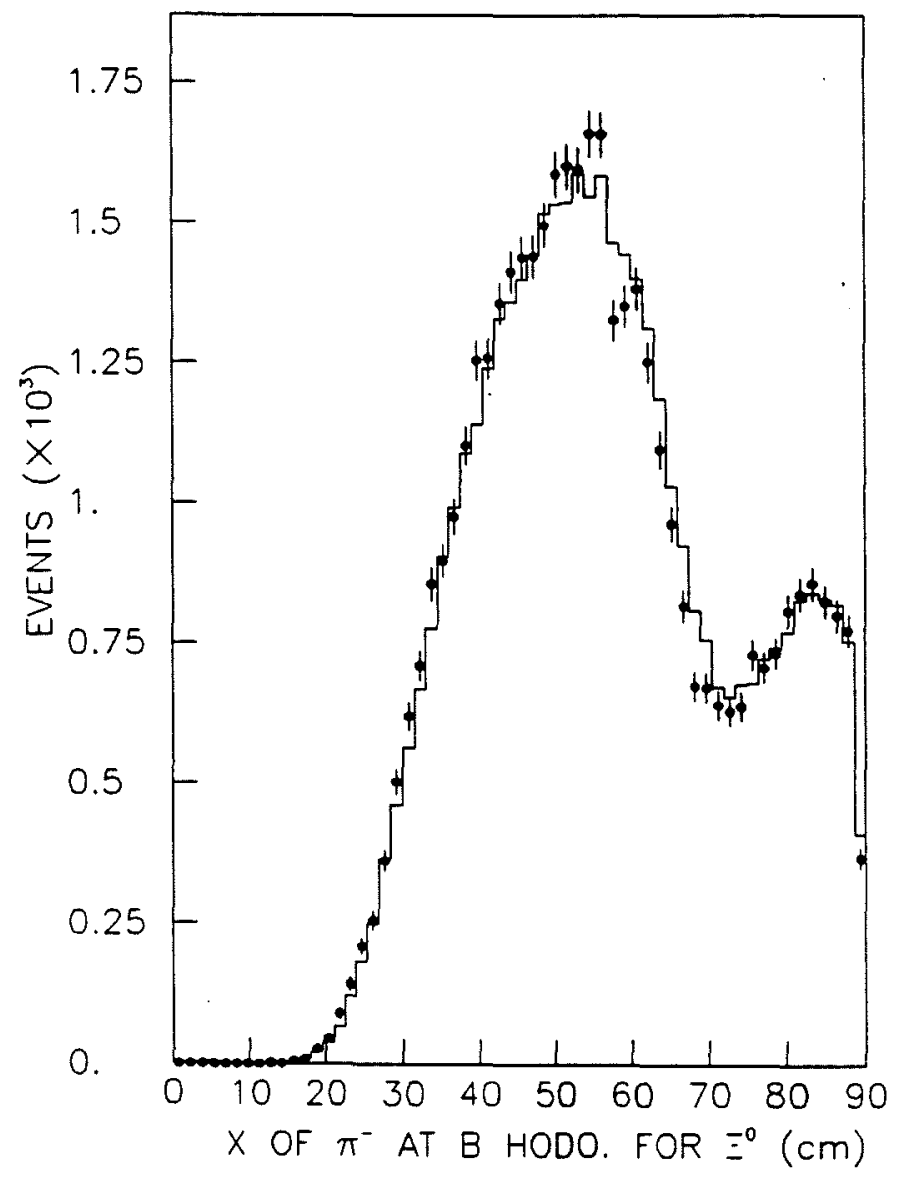

Figure 4.5: Distribution in $\mathrm{X}$ of $\pi^{-}$tracks from $\Xi^{0}$ events projected to the plane of the $\mathrm{B}$ hodoscope. Note the depletion of events centered at about $+70 \mathrm{~cm}$. (Data - dots, MC - histogram) 


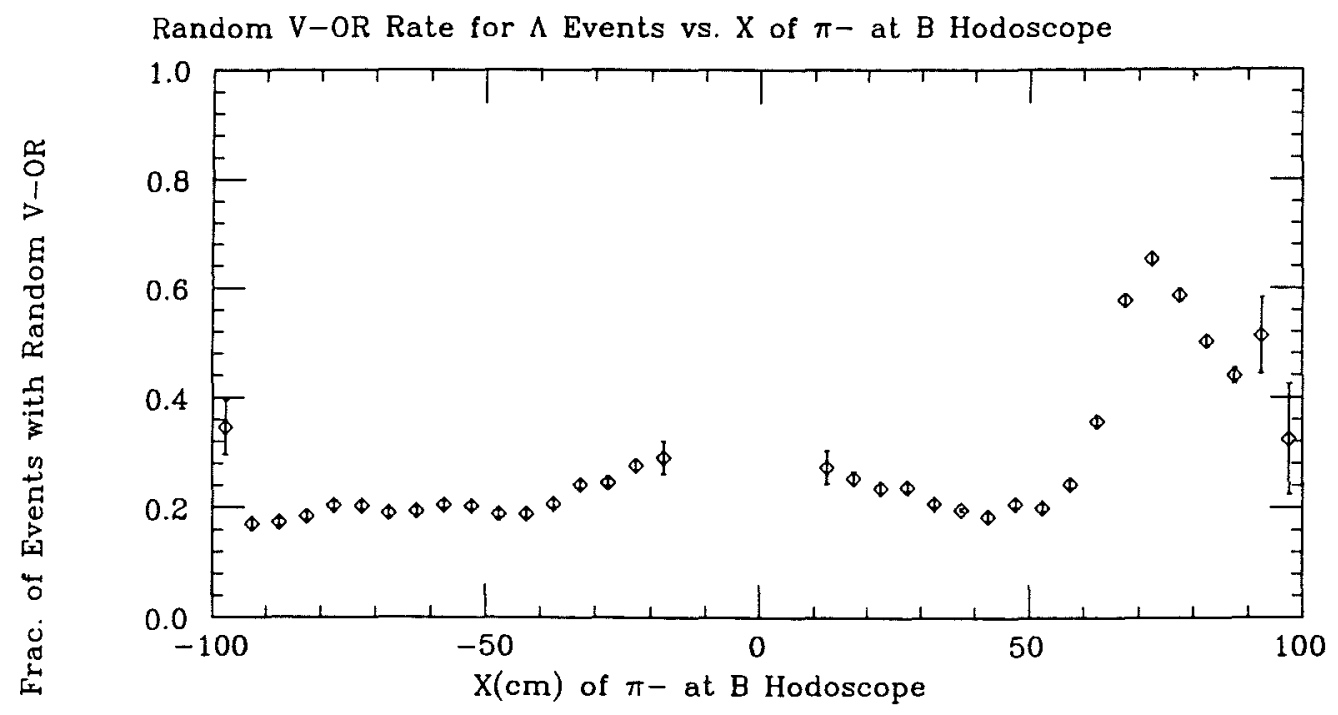

Figure 4.6: Rate of $V_{O R}$ vetoes for $\Lambda$ trigger events as a function of the $\mathrm{x}$ of the $\pi^{-}$at the $B$ hodoscope. 


\section{Chapter 5}

\section{Physics Analysis}

\subsection{Lifetime Measurements - General}

The probability that an unstable particle has not decayed after a given time $t$ is defined by the exponential decay law

$$
N(t)=N(0) \exp (-t / \tau)
$$

where $N(t)$ is the number of particles remaining at time $t$ from an initial set $N(0)$ at $t=0$. The quantity $\tau$ characterizing this relationship is the "lifetime". If this is a set of identical particles of momentum $p$ travelling along the $z$-axis, each particle travels some distance before decaying. The distance $z$ travelled from the origin is related to the time elapsed in the rest frame of the particle,

$$
z=\frac{p t}{m}
$$

where $m$ is the mass of the particle. Thus, the number of particles which travel a distance $z$ without decaying is

$$
N(p, z)=N(p, 0) \exp (-m z / p \tau)
$$

What was observed in the experiment was the number of particles $n(p, z)$ which did decay. Summed over the interval from 0 to $z$, this was $1-N(p, z)$. The change in the 
total number of decayed particles $n(p, z)$ as a function of $z$ is found by differentiating $1-N(p, z)$.

$$
\frac{d n(p, \dot{z})}{d z}=N(p, 0)\left(\frac{m}{p \tau}\right) \exp (-m z / p \tau)
$$

The decay events were summed into arrays $n\left(p_{i}, z_{j}\right)$ according to their momentum and decay vertex. $p_{i}$ and $z_{j}$ were the central momentum and decay vertex z-positions of each bin, which had finite bin widths of $\Delta p$ and $\Delta z$. The expression for the contents of a $z$-bin is found by integrating Equation 5.4 over the bin. In the limit that $m \Delta z / p_{j} \tau$ is small, then:

$$
n\left(p_{i}, z_{j}\right)=N\left(p_{i}, 0\right)\left(\frac{m \Delta z}{p_{i} \tau}\right) \exp \left(-m z_{j} / p_{i} \tau\right)
$$

\subsubsection{Lifetime Fitting Technique}

The actual number of events in each bin of the $n\left(p_{i}, z_{j}\right)$ arrays was reduced from the total number that decayed by a factor $\epsilon$ which reflected the efficiency with which decay events were observed and reconstructed. This "acceptance" was a factor in both the real $\left[\mathcal{N}_{R}\left(p_{i}, z_{j}\right)\right]$ and $\mathrm{MC}\left[\mathcal{N}_{M C}\left(p_{i}, z_{j}\right)\right]$ event arrays:

$$
\begin{gathered}
\mathcal{N}_{R}\left(p_{i}, z_{j}\right)=\epsilon_{R}\left(p_{i}, z_{j}\right) N_{R}\left(p_{i}\right) \frac{m \Delta z}{p_{i} \tau} \exp \left(-\frac{m z_{j}}{p_{i} \tau}\right) \\
\mathcal{N}_{M C}\left(p_{i}, z_{j}, \tau_{0}\right)=\epsilon_{M C}\left(p_{i}, z_{j}\right) N_{M C}\left(p_{i}\right) \frac{m \Delta z}{p_{i} \tau_{0}} \exp \left(-\frac{m z_{j}}{p_{i} \tau_{0}}\right)
\end{gathered}
$$

$\tau_{0}$ was the value for the lifetime which was used in generating the original MC sample. For the lifetime fit to be successful, the MC acceptance had to match that of the real events.

The lifetime fit was done by finding the particular value of the lifetime which minimized the difference between the $\mathrm{MC}$ and real event arrays. Rather than generate a new MC sample for each lifetime value to be tested, we weighted the MC array contents in each bin by a factor that accounted for differences caused by changing the lifetime from its initial value of $\tau_{0}[23]$. This was possible because the acceptance was a function of the momentum and decay vertex, and not of the lifetime. 
First, the weighting factors $W\left(p_{i}, z_{j}, \tau^{\prime}\right)$ for the trial lifetime $\tau^{\prime}$ were calculated.

$$
\begin{aligned}
W\left(p_{i}, z_{j}, \tau^{\prime}\right) & =\frac{\mathcal{N}_{M C}\left(p_{i}, z_{j}, \dot{\tau}^{\prime}\right)}{\mathcal{N}_{M C}\left(p_{i}, z_{j}, \tau_{0}\right)} \\
& =\frac{m \Delta z / p_{i} \tau^{\prime}}{\exp \left(-m z_{j} / p_{i} \tau^{\prime}\right)} \\
& =\frac{\tau_{0}}{\tau^{\prime}} \exp \left[-\frac{m p_{j}}{p_{i}}\left(\frac{1}{\tau^{\prime}}-\frac{1}{\tau_{0}}\right)\right]
\end{aligned}
$$

Next, the new $M C$ arrays $\mathcal{N}_{M C}\left(p_{i}, z_{j}, \tau^{\prime}\right)$ were calculated using these weights. Sums were then taken of the arrays over the $\mathrm{z}$-bins and the p-bins:

$$
\begin{array}{ll}
\mathcal{N}_{R}\left(p_{i}\right)=\sum_{j} \mathcal{N}_{R}\left(p_{i}, z_{j}\right) & \mathcal{N}_{M C}\left(p_{i}, \tau^{\prime}\right)=\sum_{j} \mathcal{N}_{M C}\left(p_{i}, z_{j}, \tau^{\prime}\right) \\
\mathcal{N}_{R}\left(z_{j}\right)=\sum_{i} \mathcal{N}_{R}\left(p_{i}, z_{j}\right) & \mathcal{N}_{M C}\left(z_{j}, \tau^{\prime}\right)=\sum_{i} \mathcal{N}_{M C}\left(p_{i}, z_{j}, \tau^{\prime}\right)
\end{array}
$$

The MC event samples had been generated with sufficient statistics that there were 4 to 5 times as many $\mathrm{MC}$ events per bin as there were real events. In order to compare them to the array of real events, the MC array had to be normalized to the real events, both to correct for the difference in statistics and minor momentum-spectrum differences. The momentum normalization factors $C\left(p_{i}, \tau^{\prime}\right)$ were calculated from the p-bin sums:

$$
C\left(p_{i}, \tau^{\prime}\right)=\mathcal{N}_{R}\left(p_{i}\right) / \mathcal{N}_{M C}\left(p_{i}, \tau^{\prime}\right)
$$

The MC arrays were multiplied bin-by-bin by the normalization factors to create the arrays $\mathcal{N}_{M C}^{\prime}\left(z_{j}, \tau^{\prime}\right)$ to be compared to the real events:

$$
\mathcal{N}_{M C}^{\prime}\left(z_{j}, \tau^{\prime}\right)=\sum_{i} C\left(p_{i}, \tau^{\prime}\right) \mathcal{N}_{M C}\left(p_{i}, z_{j}, \tau^{\prime}\right)
$$

The initial determination of the lifetime was made by minimizing a $\chi^{2}$ which had a contribution from each z-bin:

$$
\chi^{2}\left(\tau^{\prime}\right)=\sum_{j} \frac{\left[\mathcal{N}_{R}\left(z_{j}\right)-\mathcal{N}_{M C}^{\prime}\left(z_{j}, \tau^{\prime}\right)\right]^{2}}{\sigma_{j}^{2}}
$$


The variance was calculated from :

$$
\sigma_{j}^{2}=\sigma_{R}^{2}\left(z_{j}\right)+\sum_{i} C^{2}\left(p_{i}, \tau^{\prime}\right) \mathcal{N}_{M C}\left(p_{i}, \dot{z}_{j}, \tau^{\prime}\right)
$$

Where the number of events in a z-bin was great enough that the statistical uncertainty could be approximated by a Gaussian distribution, then $\sigma_{R}\left(z_{j}\right)=\sqrt{N_{R}\left(z_{j}\right)}$. Where the bin contents were low $(<20)$, the uncertainty due to Poisson statistics was approximated[23] by letting $\sigma=1.9$ if $N=0$, and $1.3+\sqrt{N}$ otherwise.

Because the bins were summed together in momentum to perform the $\chi^{2}$ fit, this method did not take full advantage of the data. The lifetime fit method was improved by using a "maximum-likelihood" method which used each bin separately in the fit.

To determine the maximum likelihood, one first determines the probability $P$ that each bin could have the observed number of events, $n_{i}$, based on an expected occupancy $\nu_{i}$, which is a function of some vector of parameters $\vec{a}$. For a Poisson distribution, assuming no uncertainty in the value of $\nu_{i}$, this probability is:

$$
P_{n_{i}}\left(\nu_{i}\right)=\frac{\left(\nu_{i}\right)^{n_{i}}}{n_{i} !} e^{-\nu_{i}}
$$

The product of these probabilities is the likelihood function $\mathcal{L}$ :

$$
\mathcal{L}(\vec{a})=\prod_{i} P_{n_{i}}\left(\nu_{i}(\vec{a})\right)
$$

The parameters are then varied to maximize the value of $\mathcal{L}$. Here, it was more convenient to deal with the negative "log-likelihood", which has a minimum at the same values of $\vec{a}$ :

$$
\begin{aligned}
L(\vec{a}) & =-2 \ln [\mathcal{L}(\vec{a})] \\
& =2 \sum_{i}\left[\nu_{i}-n_{i} \ln \left(\nu_{i}\right)\right]+\text { const. }
\end{aligned}
$$

For samples which are large enough that Gaussian statistics are valid, this $L(\vec{a})$ is equivalent to a $\chi^{2}$. The factor of 2 was added so that a difference of one in $L$ was equal to one unit of $\chi^{2}$. This allowed the $1-\sigma$ uncertainties to be calculated from the fit. 
In the lifetime fit, the $n_{i j}$ array was just $\mathcal{N}_{R}\left(p_{i}, z_{j}\right)$. The estimated occupancy $\nu_{i j}$ was determined from the MC:

$$
\begin{aligned}
\nu_{i j}\left(\tau^{\prime}, C\left(p_{i}\right)\right) & =C\left(p_{i}\right) W\left(p_{i}, z_{j}, \tau^{\prime}\right) \mathcal{N}_{M C}\left(p_{i}, z_{j}, \tau_{0}\right) \\
& =C\left(p_{i}\right) \frac{\tau_{0}}{\tau^{\prime}} \exp \left[-\frac{m z_{j}}{p_{i}}\left(\frac{1}{\tau^{\prime}}-\frac{1}{\tau_{0}}\right)\right] \mathcal{N}_{M C}\left(p_{i}, z_{j}, \tau_{0}\right)
\end{aligned}
$$

Here, the momentum spectrum normalization factors, $C\left(p_{i}\right)$, were not calculated, but were allowed to float as parameters in the likelihood maximization. The negative loglikelihood function for the lifetime fit was

$$
L\left(\tau^{\prime}, C_{i}\right)=2 \sum_{i j}\left[\nu_{i j}\left(\tau^{\prime}, C_{i}\right)-\mathcal{N}_{R}\left(p_{i}, z_{j}\right) \ln \left(\nu_{i j}\left(\tau^{\prime}, C_{i}\right)\right)\right]+\text { const. }
$$

The maximum-likelihood fit gave results consistent with those from the $\chi^{2}$ fit.

\section{2 $\Lambda$ Lifetime Fits}

The beam $\Lambda$ data was divided into sets both by trigger (F.C5 and $\Lambda$ ) and by target (Downstream-East, and Downstream-West) The samples from the two triggers were used in separate determinations of the $\Lambda$ lifetime, and then the results compared as a check of systematics due to different acceptances.

\subsection{1 $\tau_{\Lambda}$ from F.C5 Trigger}

The F.C5 data and MC events were reconstructed using the same cuts, as was described in the previous chapter. The events were then put into $\left(P_{\Lambda}, Z_{\Lambda}\right)$ bins using the results of the kinematic $\Lambda$ fit. The momentum bins were $10 \mathrm{GeV} / \mathrm{c}$ wide, and ranged from 90-530 GeV/c, while the vertex bins were $0.5 \mathrm{~m}$ wide, ranging from 2.0 to 19.0 meters downstream of the exit of the collimator. This restricted vertex range ensured that the events fell within the decay volume defined by the V1 and DK counters. Fits were then made over different momentum ranges and targets. The results using the DE and DW target events separately are presented in Table 5.1. The events from the DE and DW 


\begin{tabular}{|c|c|rcc|rcc|}
\hline$P_{\Lambda}$ Bin & Avg. $P_{\Lambda}$ & \multicolumn{3}{|c|}{ DE Target } & \multicolumn{3}{|c|}{ DW Target } \\
$(\mathrm{GeV} / \mathrm{c})$ & $(\mathrm{GeV} / \mathrm{c})$ & Events & $c \tau_{\Lambda}(\mathrm{cm})$ & $\chi^{2}$ & Events & $c \tau_{\Lambda}(\mathrm{cm})$ & $\chi^{2}$ \\
\hline$<110$ & 102 & 1268 & $7.43 \pm 0.35$ & 28.4 & 1345 & $7.96 \pm 0.38$ & 14.5 \\
$110-170$ & 145 & 10699 & $\mathbf{8 . 2 2} \pm 0.19$ & 29.3 & 11619 & $8.37 \pm 0.19$ & 18.8 \\
$170-230$ & 201 & 17977 & $7.55 \pm 0.17$ & 27.4 & 19920 & $7.95 \pm 0.18$ & 28.2 \\
$230-290$ & 260 & 20354 & $7.72 \pm 0.21$ & 45.4 & 22606 & $7.83 \pm 0.21$ & 28.1 \\
$290-350$ & 319 & 19181 & $7.73 \pm 0.28$ & 32.8 & 21656 & $7.36 \pm 0.23$ & 41.7 \\
$350-410$ & 379 & 15362 & $7.15 \pm 0.32$ & 36.4 & 17603 & $8.10 \pm 0.39$ & 19.8 \\
$410-470$ & 439 & 11682 & $7.40 \pm 0.45$ & 34.2 & 13395 & $7.39 \pm 0.43$ & 27.8 \\
$470-530$ & 498 & 8051 & $8.23 \pm 0.80$ & 37.0 & 9707 & $7.63 \pm 0.63$ & 24.3 \\
\hline $110-410$ & 269 & 83573 & $7.78 \pm 0.10$ & 39.5 & 93404 & $7.99 \pm 0.10$ & 39.7 \\
$110-470$ & 290 & 95255 & $7.76 \pm 0.09$ & 43.8 & 106799 & $7.97 \pm 0.09$ & 39.2 \\
\hline
\end{tabular}

Table 5.1: Fits of $c \tau_{\Lambda}$ for DE and DW target F.C5 trigger data. The $\chi^{2}$ is a sum over $34 \mathrm{Z}$ bins.

targets were then taken together in a combined fit (Table 5.2). Figure 5.1 compares the lifetime from the different momentum ranges. The relatively large $\chi^{2}$ for the overall fit was due mostly to the difference between the lifetimes measured with the DE and DW target event samples.

\section{Systematic Errors}

The errors quoted above were due only to statistical uncertainties. The results were also analyzed for the presence of systematic biases which would increase the uncertainty of the lifetime measurement. Sources considered were the choice of $p$ and $z$ bin sizes, remaining backgrounds, and the sensitivity to the selection criteria.

Table 5.2 shows that changing the end of the $\Lambda$ momentum range at $410 \mathrm{GeV} / \mathrm{c}$ by $60 \mathrm{GeV} / \mathrm{c}$ caused a $\pm 0.02 \mathrm{~cm}$ shift in $c \tau_{\Lambda}$. Changing the size of the z-bins and p-bins had no significant effect on the fits. Any remaining background events of $\Lambda$ 's which were produced or scattered in the walls of the neutral beam collimator had no detrimental effect on the lifetime measurement, because they were all produced upstream of the decay volume, and thus were merely an additional source of events for the lifetime fit. If a significant number of background events of $\Lambda$ 's from $\Xi^{0}$ decays 


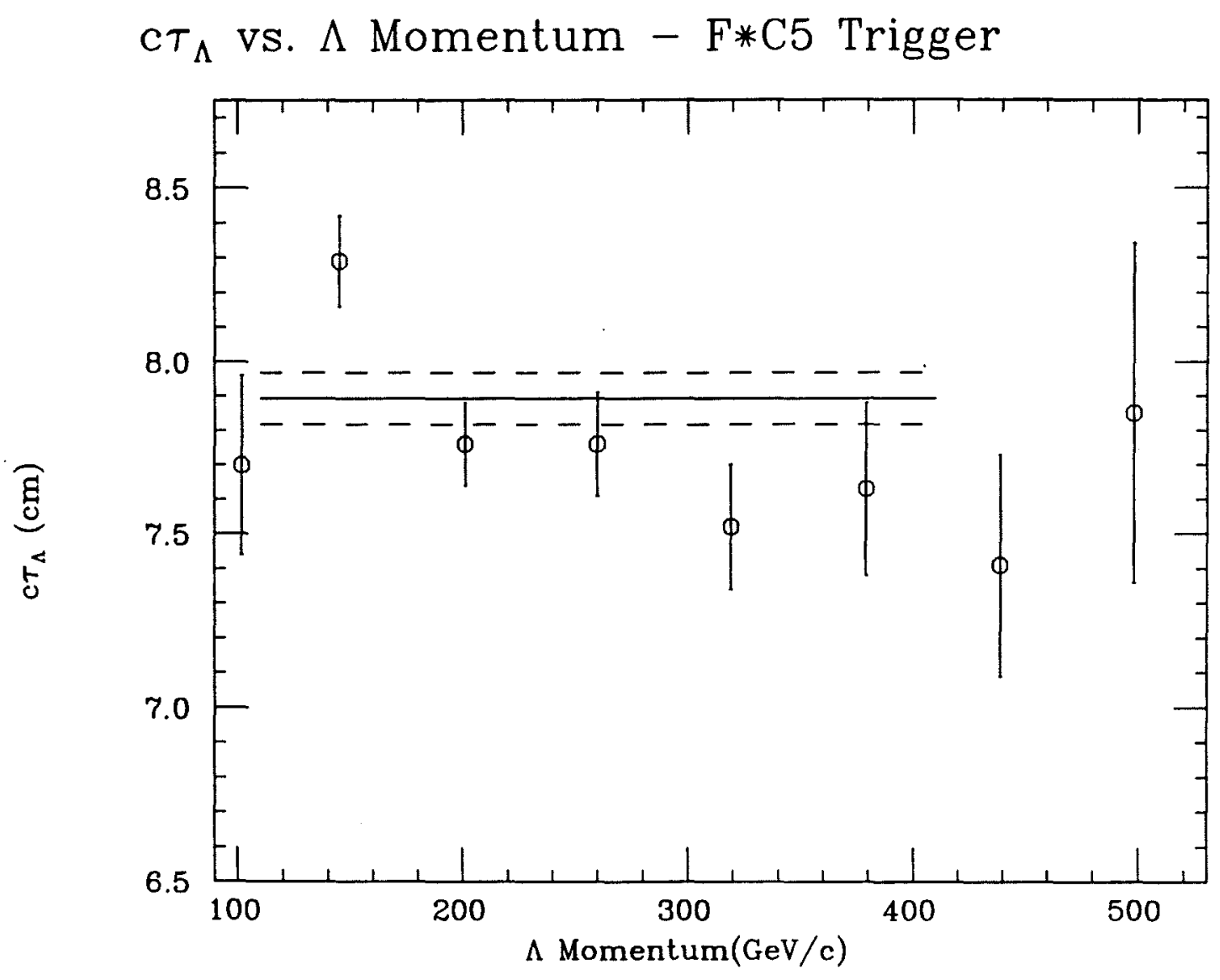

Figure 5.1: Momentum dependence of $c \tau_{\Lambda}$ for F.C5 trigger events. The solid and dashed lines indicate the average $c \tau_{\Lambda}$ of $7.89 \pm 0.07$ over the indicated momentum range of $110-410 \mathrm{GeV} / \mathrm{c}$. 


\begin{tabular}{|c|c|rcc|}
\hline $\begin{array}{c}P_{\Lambda} \text { Bin } \\
(\mathrm{GeV} / \mathrm{c})\end{array}$ & $\begin{array}{c}\text { Avg. } P_{\Lambda} \\
(\mathrm{GeV} / \mathrm{c})\end{array}$ & \multicolumn{3}{|c|}{$\mathrm{DE}+\mathrm{DW}$} \\
\hline $90-110$ & 102 & 2613 & $7.70 \pm 0.26$ & $\mathbf{2 0 . 6}$ \\
$110-170$ & 145 & 22318 & $8.29 \pm 0.13$ & $\mathbf{2 4 . 8}$ \\
$170-230$ & 201 & 37897 & $7.76 \pm 0.12$ & 36.2 \\
$230-290$ & 260 & 42960 & $7.76 \pm 0.15$ & 48.6 \\
$290-350$ & 319 & 40837 & $7.52 \pm 0.18$ & 45.7 \\
$350-410$ & 379 & 32965 & $7.63 \pm 0.25$ & $\mathbf{2 5 . 4}$ \\
$410-470$ & 439 & 25077 & $7.41 \pm 0.32$ & 37.6 \\
$470-530$ & 498 & 17758 & $7.85 \pm 0.49$ & 34.7 \\
\hline $110-350$ & 268 & 144012 & $7.91 \pm 0.07$ & $\mathbf{5 2 . 7}$ \\
$110-410$ & 268 & 176977 & $7.89 \pm 0.07$ & $\mathbf{5 4 . 6}$ \\
$110-470$ & 289 & 202054 & $7.87 \pm 0.07$ & $\mathbf{5 7 . 6}$ \\
$110-530$ & 268 & 219812 & $7.87 \pm 0.07$ & 48.9 \\
\hline
\end{tabular}

Table 5.2: Fits of $c \tau_{\Lambda}$ for combined F.C5 trigger data. Again, the $\chi^{2}$ is the total for 34 $\mathrm{Z}$ bins.

existed, they would bias the lifetime fit towards larger values of $c \tau_{\Lambda}$, because their vertex distributions (Figure 3.18) were peaked much farther downstream than those of the beam $\Lambda$ 's (Figure 3.11). These had been removed by the restrictions on $R_{\Lambda}^{2}, \Delta \Theta_{x}$ , $\Delta \Theta_{y}$, and requiring no photon showers in the lead glass array. The sensitivity to the selection criteria was studied by preparing alternative real and MC event samples using more stringent selection criteria, and then observing what effect these changes had on the fitted value for the lifetime. Three cases were analyzed, with the resulting fitted lifetimes presented in Table 5.3, as well as their difference from the results above (Table 5.2) for the $110-410 \mathrm{GeV} / \mathrm{c}$ momentum range. In the first case, the maximum $R_{\Lambda}^{2}$ of $0.25 \mathrm{~cm}^{2}$ was reduced by $0.025 \mathrm{~cm}^{2}$ and the maximum $\Delta \Theta_{x}$ and $\Delta \Theta_{y}$ were reduced by 0.0005 . In the second case, a wider track separation was required with the combined separation in the $\mathrm{x}$-plane at chambers $\mathrm{C} 1$ and $\mathrm{C} 3$ being $>1.0 \mathrm{~cm}$ and in the $y$-plane $>0.2 \mathrm{~cm}$. In the third case, the fiducial volume in $\mathrm{x}$ and $\mathrm{y}$ at chambers $\mathrm{C} 4$ and $\mathrm{C5}$ that the charged particles had to pass through was reduced by $1 \mathrm{~cm}$ in each dimension. The results in Table 5.3 show a maximum possible systematic deviation of $\pm 0.02 \mathrm{~cm}$. This result was combined with the $0.02 \mathrm{~cm}$ deviation due to changes in the momentum 


\begin{tabular}{|l|c|c|c|c|}
\hline Additional Criteria & Events & $c \tau_{\Lambda}(\mathrm{cm})$ & $\chi^{2}$ & Deviation $(\mathrm{cm})$ \\
\hline Smaller $R_{\Lambda}^{2}, \Delta \Theta_{x}, \Delta \Theta_{y}$ & 173463 & $7.87 \pm 0.07$ & 55.6 & -0.02 \\
Wider Track Separation & 174805 & $7.89 \pm 0.07$ & 54.2 & 0 \\
Smaller MWPC Apertures & 175498 & $7.87 \pm 0.07$ & $\mathbf{5 5 . 1}$ & -0.02 \\
\hline
\end{tabular}

Table 5.3: Systematic variation of $c \tau_{\Lambda}$ from F.C5 trigger data, due to changes in the selection criteria.

range for an estimated systematic error of $\pm 0.04 \mathrm{~cm}$. Thus the final result for the F.C5 trigger data set was:

$$
c \tau_{\Lambda}=7.89 \pm 0.07(\text { statistical }) \pm 0.04(\text { systematic }) \mathrm{cm}
$$

If the discrepancy in $c \tau_{\Lambda}$ between the DE and DW events (Table 5.1) was not just a statistical fluctuation, but an actual difference, then the error in the combined $c \tau_{\Lambda}$ should be increased.

\subsection{2 $\tau_{\Lambda}$ from $\Lambda$ Trigger}

The $\Lambda$ trigger events were treated in a similar way to the F.C5 data. The selection criteria used differed slightly to ensure that events fell well within the limits of the $A$ and B trigger hodoscopes. Fits for the DE and DW target events separately are presented in Table 5.4, and for the combined DE and DW target data in Table 5.5. Figure 5.2 compares the lifetime from the different momentum ranges. The only significant systematic deviation occurs above $410 \mathrm{Gev} / \mathrm{c}$. This deviation is probably because at high momentum the average opening angle between the proton and $\pi^{-}$are so small that the acceptance becomes a strong function of the vertex position. This results in a vertex distribution which is skewed towards low values of $z$, an effect which was apparently not totally modelled by the MC. Because of this systematic deviation, the data above $410 \mathrm{GeV} / \mathrm{c}$ was not used in the final average. This same momentum range of $110-410$ $\mathrm{GeV} / \mathrm{c}$ was also used for the F.C5 trigger data covered above. Even if this data had been used, it would only change the overall average by $-0.01 \mathrm{~cm}$. 


\begin{tabular}{|c|c|rcc|rcc|}
\hline$P_{\Lambda}$ bin & Avg. $P_{\Lambda}$ & \multicolumn{3}{|c|}{ DE Target } & \multicolumn{3}{c|}{ DW Target } \\
$(\mathrm{GeV} / \mathrm{c})$ & $(\mathrm{GeV} / \mathrm{c})$ & Events & $c \tau_{\Lambda}(\mathrm{cm})$ & $\chi^{2}$ & Events & $c \tau_{\Lambda}(\mathrm{cm})$ & $\chi^{2}$ \\
\hline $90-110$ & 105 & 1134 & $\mathbf{8 . 7 4} \pm \mathbf{0 . 5 3}$ & $\mathbf{3 3 . 5}$ & $\mathbf{1 3 2 2}$ & $7.67 \pm 0.39$ & $\mathbf{2 7 . 2}$ \\
$110-170$ & 146 & $\mathbf{2 6 6 9 4}$ & $7.95 \pm \mathbf{0 . 1 1}$ & $\mathbf{2 1 . 9}$ & $\mathbf{2 8 3 0 0}$ & $7.93 \pm \mathbf{0 . 1 1}$ & $\mathbf{2 7 . 1}$ \\
$170-230$ & 201 & 49936 & $8.00 \pm \mathbf{0 . 1 2}$ & 29.6 & 54151 & $7.79 \pm 0.10$ & $\mathbf{2 3 . 0}$ \\
$\mathbf{2 3 0 - 2 9 0}$ & 260 & 57767 & $7.62 \pm \mathbf{0 . 1 3}$ & $\mathbf{3 7 . 5}$ & 63024 & $7.71 \pm \mathbf{0 . 1 2}$ & $\mathbf{2 9 . 1}$ \\
$\mathbf{2 9 0 - 3 5 0}$ & $\mathbf{3 1 9}$ & $\mathbf{5 6 1 1 3}$ & $7.84 \pm \mathbf{0 . 1 7}$ & $\mathbf{3 2 . 4}$ & 62595 & $7.90 \pm \mathbf{0 . 1 6}$ & $\mathbf{3 1 . 8}$ \\
$\mathbf{3 5 0 - 4 1 0}$ & $\mathbf{3 7 8}$ & $\mathbf{4 3 3 9 8}$ & $7.72 \pm \mathbf{0 . 2 2}$ & $\mathbf{2 0 . 4}$ & 48346 & $8.13 \pm 0.23$ & $\mathbf{3 2 . 8}$ \\
$410-470$ & 436 & $\mathbf{2 2 7 6 7}$ & $7.39 \pm \mathbf{0 . 3 4}$ & $\mathbf{2 1 . 3}$ & $\mathbf{2 6 3 0 0}$ & $\mathbf{7 . 5 2} \pm \mathbf{0 . 3 2}$ & $\mathbf{2 1 . 2}$ \\
$470-530$ & 495 & 8452 & $\mathbf{6 . 7 7} \pm \mathbf{0 . 5 5}$ & $\mathbf{2 4 . 3}$ & 10673 & $7.01 \pm \mathbf{0 . 5 2}$ & $\mathbf{1 6 . 7}$ \\
\hline $110-410$ & 271 & $\mathbf{2 3 3 9 0 8}$ & $7.87 \pm \mathbf{0 . 0 6}$ & 40.6 & $\mathbf{2 5 6 4 1 6}$ & $\mathbf{7 . 8 5} \pm \mathbf{0 . 0 6}$ & $\mathbf{2 8 . 8}$ \\
$110-470$ & 286 & 256675 & $7.85 \pm \mathbf{0 . 0 6}$ & $\mathbf{3 9 . 2}$ & $\mathbf{2 8 2 7 1 6}$ & $7.84 \pm \mathbf{0 . 0 6}$ & $\mathbf{2 5 . 8}$ \\
\hline
\end{tabular}

Table 5.4: Fits of $c \tau_{\Lambda}$ for DE and DW target $\Lambda$ trigger events. The $\chi^{2}$ is the total for $34 \mathrm{Z}$ bins.

\begin{tabular}{|c|c|rcc|}
\hline$P_{\Lambda}$ Bin & Avg. $P_{\Lambda}$ & \multicolumn{3}{|c|}{ DE+DW Targets } \\
$(\mathrm{GeV} / \mathrm{c})$ & $(\mathrm{GeV} / \mathrm{c})$ & Events & $c \tau_{\Lambda}(\mathrm{cm})$ & $\chi^{2}$ \\
\hline $90-110$ & 105 & 2456 & $8.13 \pm 0.31$ & 28.8 \\
$110-170$ & 146 & 54994 & $7.94 \pm 0.08$ & 31.8 \\
$170-230$ & 201 & 104087 & $7.89 \pm 0.08$ & 21.0 \\
$230-290$ & 260 & 120791 & $7.66 \pm 0.09$ & 31.9 \\
$290-350$ & 320 & 118708 & $7.87 \pm 0.12$ & 29.6 \\
$350-410$ & 378 & 91744 & $7.93 \pm 0.16$ & 29.8 \\
$410-470$ & 436 & 49067 & $7.45 \pm 0.23$ & 22.4 \\
$470-530$ & 495 & 19125 & $6.90 \pm 0.37$ & 21.7 \\
\hline $110-350$ & 246 & 398580 & $7.85 \pm 0.04$ & 26.9 \\
$110-410$ & 271 & 490324 & $7.86 \pm 0.04$ & 35.6 \\
$110-470$ & 286 & 539391 & $7.85 \pm 0.04$ & 30.9 \\
$110-530$ & 293 & 558516 & $7.84 \pm 0.04$ & 28.6 \\
\hline
\end{tabular}

Table 5.5: Fits of $c \tau_{\Lambda}$ for combined $\Lambda$ trigger data. The $\chi^{2}$ is over $34 \mathrm{Z}$ bins. 


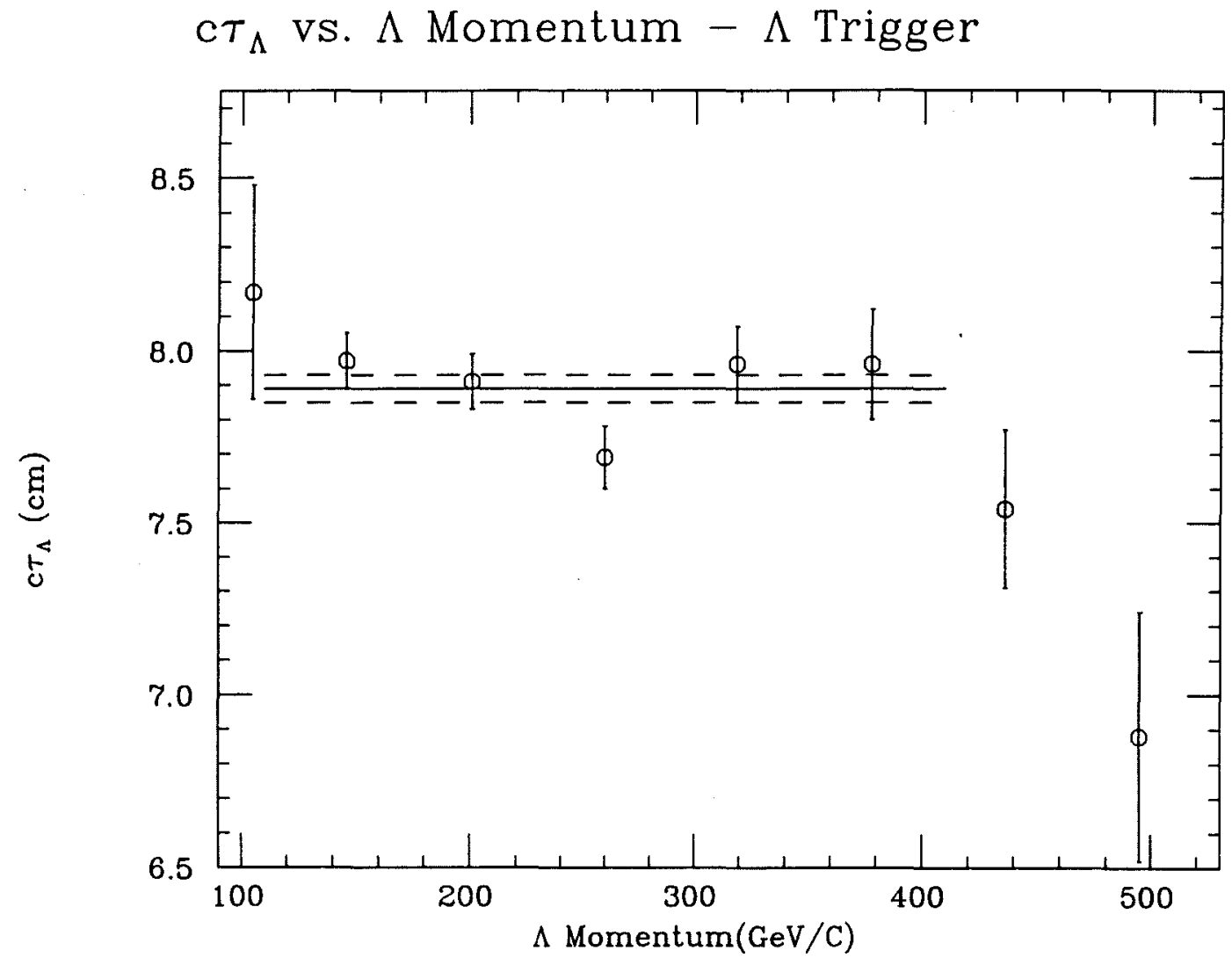

Figure 5.2: Momentum dependence of $c \tau_{\Lambda}$ for $\Lambda$ trigger events. The solid and dashed lines indicate the average $c \tau_{\Lambda}$ of $7.86 \pm 0.04$ over the indicated momentum range of $110-410 \mathrm{GeV} / \mathrm{c}$. 


\begin{tabular}{|l|c|c|c|c|}
\hline Additional Criteria & Events & $c \tau_{\Lambda}(\mathrm{cm})$ & $\chi^{2}$ & Deviation $(\mathrm{cm})$ \\
\hline Smaller $R_{\Lambda}^{2}, \Delta \Theta_{x}, \Delta \Theta_{y}$ & 480661 & $7.86 \pm 0.04$ & 35.3 & 0.0 \\
Wider Track Separation & 483786 & $7.87 \pm 0.04$ & $\mathbf{3 5 . 6}$ & +0.01 \\
Smaller Fiducial Volume & 470894 & $7.85 \pm 0.04$ & $\mathbf{3 2 . 9}$ & -0.01 \\
\hline
\end{tabular}

Table 5.6: Systematic variation of $c \tau_{\Lambda}$ from $\Lambda$ trigger data due to changes in the selection criteria

\section{Systematic Errors}

A systematic error analysis was made of the $\Lambda$ trigger data as had been done with the F.C5 trigger sample. The value of $c \tau_{\Lambda}$ did not vary with reductions in the maximum allowed $Z_{\Lambda}$ of $19.0 \mathrm{~m}$. The variations in the result for $c \tau_{\Lambda}$ due to changes in the selection criteria are shown in Table 5.6. The first and second cases had the same changes in maximum $R_{\Lambda}^{2}, \Delta \Theta_{x}, \Delta \Theta_{y}$ and track separation as for the F.C5 event analysis. In the third case, the fiducial volume criteria used the positions at the $\mathrm{A}$ and $\mathrm{B}$ trigger hodoscopes and the proton counters instead of at the MWPC's. Based on the $\pm 0.01 \mathrm{~cm}$ maximum systematic deviation from Table 5.6, and the small $0.01 \mathrm{~cm}$ variation with the change in momentum range, the systematic error was estimated to be $\pm 0.02 \mathrm{~cm}$. Thus the final result for the $\Lambda$ trigger data set was:

$$
c \tau_{\Lambda}=7.86 \pm 0.04(\text { statistical }) \pm 0.02(\text { systematic }) \mathrm{cm}
$$

The F.C5 trigger data fit result of $c \tau_{\Lambda}=7.89 \pm 0.07 \pm 0.04 \mathrm{~cm}$ was consistent with this result for the $\Lambda$ trigger data. The weighted average of the two results, after combining the statistical and systematic error for each set in quadrature, was

$$
c \tau_{\Lambda}=7.87 \pm 0.04(\text { statistical }+ \text { systematic }) \mathrm{cm}
$$

The F.C5 and $\Lambda$ trigger data were also averaged bin-by-bin in momentum, with the results presented in Table 5.7. Expressed in units of time only, the result was:

$$
\left.\tau_{\Lambda}=2.62 \pm 0.01 \text { (statistical }+ \text { systematic }\right) \times 10^{-10} \mathrm{~s}
$$




\begin{tabular}{|c|c|rc|}
\hline$P_{\Lambda}$ Bin & Avg. $P_{\Lambda}$ & \multicolumn{2}{|c|}{ DE+DW Targets } \\
$(\mathrm{GeV} / \mathrm{c})$ & $(\mathrm{GeV} / \mathrm{c})$ & Events & $c \tau_{\Lambda}(\mathrm{cm})$ \\
\hline $90-110$ & 104 & 5069 & $7.88 \pm 0.20$ \\
$110-170$ & 146 & 77312 & $8.04 \pm 0.07$ \\
$170-230$ & 201 & 141984 & $7.85 \pm \mathbf{0 . 0 7}$ \\
$230-290$ & 260 & 163751 & $7.69 \pm 0.08$ \\
$290-350$ & 320 & 159545 & $7.76 \pm 0.10$ \\
$350-410$ & 378 & 124709 & $7.84 \pm \mathbf{0 . 1 3}$ \\
$410-470$ & 437 & 74144 & $7.44 \pm 0.19$ \\
$470-530$ & 496 & 36883 & $7.24 \pm 0.30$ \\
\hline $110-410$ & 270 & 667301 & $7.87 \pm 0.03$ \\
\hline
\end{tabular}

Table 5.7: Weighted average of $c \tau_{\Lambda}$ for F.C5 and $\Lambda$ trigger data. Only the statistical errors are included here.

\section{$5.3 \Xi^{0}$ Lifetime Fit}

For the $\Xi^{0}$ lifetime measurement, the $\Xi^{0}$ decay events were binned according to the $\Xi^{0}$ momentum $\left(P_{\Xi}\right)$ and decay vertex $\left(Z_{\Xi}\right)$. The fit then proceeded as was described above. Because of possible systematic errors with using the $\Xi^{0}$ vertex distribution, a alternate fit was investigated which used the distributions of the daughter $\Lambda$ 's, where the resolution on the vertex was much better. However, the resulting mathematical uncertainty in the $\Xi^{0}$ lifetime from the daughter $\Lambda$ method was inferior to that from the direct measurement.

\subsection{1 $\Xi^{0}$ Lifetime Using $\Xi^{0}$ Vertex}

The data and MC events were put in arrays according to their reconstructed $\Xi^{0}$ momentum and decay vertex. The momentum bins were $10 \mathrm{GeV} / \mathrm{c}$ wide, and ranged from $110 \mathrm{GeV} / \mathrm{c}$ to $440 \mathrm{GeV} / \mathrm{c}$. The decay vertex was required be between 0.0 and 19.0 $m$ downstream of the end of the collimator. In addition, the $\Lambda$ vertex was required to be between 2.0 and $19.0 \mathrm{~m}$ downstream of the collimator. The MC sample used was generated with the existing world averages of $c \tau_{\Lambda}=7.89 \mathrm{~cm}$ and $c \tau_{\Xi}=8.69 \mathrm{~cm}$ respectively. 


\begin{tabular}{|c|c|rcc|rcc|}
\hline $\begin{array}{c}P_{\Xi} \text { bin } \\
(\mathrm{GeV} / \mathrm{c})\end{array}$ & $\begin{array}{c}\text { Avg. } P_{\Xi} \\
(\mathrm{GeV} / \mathrm{c})\end{array}$ & \multicolumn{3}{|c|}{ All DE } & \multicolumn{3}{|c|}{ All DW } \\
\hline $110-140$ & 133 & 385 & $8.30 \pm 0.81$ & 7.1 & 443 & $9.30 \pm 0.96$ & 7.9 \\
$140-200$ & 176 & 8755 & $9.57 \pm 0.29$ & 12.2 & 9355 & $8.71 \pm 0.24$ & 11.7 \\
$220-260$ & 230 & 16137 & $8.95 \pm 0.25$ & 15.9 & 17635 & $9.05 \pm 0.25$ & 15.5 \\
$260-320$ & 287 & 11998 & $8.99 \pm 0.38$ & 13.9 & 12972 & $8.00 \pm 0.29$ & 17.1 \\
$320-380$ & 345 & 5356 & $8.92 \pm 0.77$ & 11.7 & 5889 & $9.28 \pm 0.73$ & 20.2 \\
$380-440$ & 403 & 1520 & $8.21 \pm 1.45$ & 5.8 & 1636 & $8.49 \pm 1.32$ & 19.4 \\
\hline $140-380$ & 248 & 42246 & $9.18 \pm 0.17$ & 18.5 & 45851 & $8.73 \pm 0.15$ & 19.8 \\
$140-440$ & 254 & 43766 & $9.17 \pm 0.17$ & 19.5 & 47847 & $8.73 \pm 0.15$ & 18.1 \\
\hline
\end{tabular}

Table 5.8: Fits of $c \tau_{\Xi}$ from $\Xi^{0}$ vertex separated by target. The $\chi^{2}$ is over $19 \mathrm{Z}$ bins.

\begin{tabular}{|c|c|rcc|}
\hline$P_{\Xi}$ bin & Avg. $P_{\Xi}$ & \multicolumn{3}{|c|}{$\mathrm{DE}+\mathrm{DW}$} \\
$(\mathrm{GeV} / \mathrm{c})$ & $(\mathrm{GeV} / \mathrm{c})$ & Events & $c T_{\Xi}(\mathrm{cm})$ & $\chi^{2}$ \\
\hline $110-140$ & 133 & 828 & $8.79 \pm 0.73$ & 6.0 \\
$140-200$ & 176 & 18110 & $9.11 \pm 0.18$ & 13.2 \\
$220-260$ & 230 & 33772 & $9.00 \pm 0.23$ & 17.0 \\
$260-320$ & 287 & 24970 & $8.45 \pm 0.30$ & 24.3 \\
$320-380$ & 345 & 11245 & $9.14 \pm 0.58$ & 11.0 \\
$380-440$ & 403 & 3156 & $8.36 \pm 1.16$ & 13.3 \\
\hline $140-380$ & 248 & 88097 & $8.94 \pm 0.11$ & 18.9 \\
$140-440$ & 254 & 91253 & $8.94 \pm 0.11$ & 19.0 \\
\hline
\end{tabular}

Table 5.9: Fits of $c \tau \Xi$ from $\Xi^{0}$ vertex for the combined DE and DW target data. The $\chi^{2}$ is over $19 \mathrm{Z}$ bins.

The results for $c \tau_{\Xi}$ separated by target are presented in Table 5.8. The $c \tau_{\Xi}$ fit result with the DE and DW target event samples combined are listed in Table 5.9. Figure 5.3 compares the lifetime from the different momentum ranges. There was no significant deviation from the average $c T \Xi$ of $8.94 \pm 0.11 \mathrm{~cm}$.

\section{Systematic Errors}

For the $\Xi^{0}$ events, several cases were evaluated in order to reveal any systematic biases in $c \tau_{\Xi}$ (Table 5.10). In the first case, only events without a photon near the hole were used. In the second case, the neutral beam selection criteria were strengthened to check for beam $\Lambda$ backgrounds. The minimum $R_{\Lambda}^{2}$ of $0.15 \mathrm{~cm}^{2}$ was increased by $0.025 \mathrm{~cm}$, 


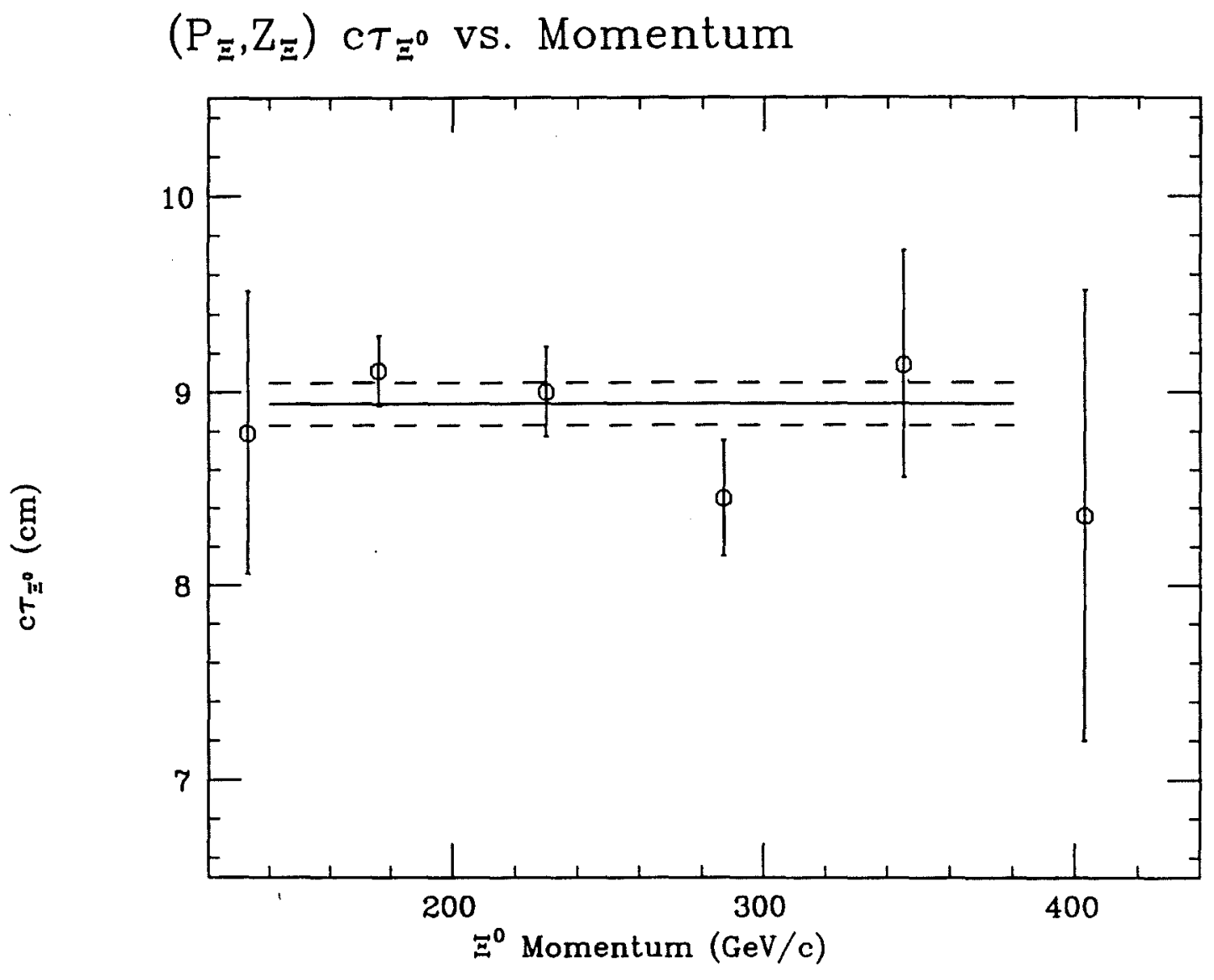

Figure 5.3: Momentum dependence of $c \tau_{\Xi}$ for $\Xi^{0}$ data. The solid and dashed lines indicate the average $c T_{\Xi}$ of $8.94 \pm 0.11 \mathrm{~cm}$ over the indicated momentum range of $140-380 \mathrm{GeV} / \mathrm{c}$. 


\begin{tabular}{|l|c|c|c|c|}
\hline Additional Criteria & Events & $c \tau_{\Xi}(\mathrm{cm})$ & $\chi^{2}$ & Deviation $(\mathrm{cm})$ \\
\hline No hole $\gamma^{\prime}$ s & 55316 & $8.87 \pm 0.15$ & 24.7 & -0.07 \\
Greater $R_{\Lambda}^{2}$, Smaller $\Delta X_{G L}, \Delta Y_{G L}$ & 85000 & $8.96 \pm 0.11$ & 17.8 & +0.02 \\
Smaller $\chi^{2}$ & 86918 & $8.98 \pm 0.11$ & 20.6 & +0.04 \\
$0.2 \%$ Smaller Gain Constants & 88097 & $8.98 \pm 0.11$ & 18.5 & +0.04 \\
\hline
\end{tabular}

Table 5.10: Systematic variation of $c r_{\Xi}$ from $\Xi^{0}$ vertex fit, due to changes in the selection criteria.

while the positions of the projected $\Xi^{0}$ at the lead glass, $X_{G L}$ and $Y_{G L}$, were required to be $0.25 \mathrm{~cm}$ closer to the neutral beam axis. In the third case, the maximum $\chi^{2}$ of the $\Xi^{0}$ fit of 40 was reduced to 37 . In the fourth case, the MC was reconstructed with the gain constants of the lead glass signals reduced by $0.2 \%$ to check the sensitivity to the lead glass calibration. Any difference in relative gain between the MC and data samples which was $>0.2 \%$ would have been seen as a significant shift in the average reconstructed $\pi^{0}$ mass. Based on these results and the absence of momentum dependence mentioned above, the possible systematic error was estimated to be \pm 0.07 cm. Thus the final result for $c T_{\Xi}$ was:

$$
\left.c \tau_{\Xi^{0}}=8.94 \pm 0.11 \text { (statistical }\right) \pm 0.07(\text { systematic }) \mathrm{cm}
$$

Expressing this in units of time, the result was:

$$
\tau_{\Xi^{0}}=2.98 \pm 0.04(\text { statistical }) \pm 0.02(\text { systematic }) \times 10^{-10_{s}}
$$

\subsection{2 $\Xi^{0}$ Lifetime Using $\Lambda$ Vertex}

Previous experiments[15] which were unable to reconstruct the $\Xi^{0}$ decay vertex for their $\Xi^{0}$ events have used the distribution of the daughter $\Lambda$ vertices, in conjunction with the reconstructed momentum of the $\Xi^{0}$ 's.

To determine the functional form of the observed distribution $\mathcal{N}_{\Lambda}\left(p_{\Xi}, p_{\Lambda}, z\right)$, let $s$ be the distance from the target to the $\Xi^{0}$ decay vertex, while $z$ is the distance from the 
target to the $\Lambda$ decay vertex. The distribution of $\Xi^{0}$ particles observed to decay at $s$ is:

$$
d \mathcal{N}_{\Xi}\left(p_{\Xi}, p_{\Lambda}, s\right)=N_{\Xi}\left(p_{\Xi}, p_{\Lambda}, 0\right) \lambda_{\Xi} \exp \left(-\lambda_{\Xi} s\right) d s
$$

where $\lambda_{\Xi}=m_{\Xi} / p_{\Xi} \tau_{\Xi}$. This distribution defines the distribution of $\Lambda$ 's observed to decay as a function of $z$ :

$$
\delta \mathcal{N}_{\Lambda}\left(p_{\Xi}, p_{\Lambda}, s, z\right)=d \mathcal{N}_{\Xi}\left(p_{\Xi}, p_{\Lambda}, s\right) \lambda_{\Lambda} \exp \left[-\lambda_{\Lambda}(z-s)\right] d z
$$

where $\lambda_{\Lambda}$ has the expected definition. To find the total distribution of events as a function of $z$, one integrates $\delta \mathcal{N}_{\Lambda}$ from $s=0$ to $z$, and applies the acceptance $\epsilon\left(p_{\Xi}, p_{\Lambda}, z\right)$ :

$$
\begin{aligned}
& d \mathcal{N}_{\Lambda}\left(p_{\Xi}, p_{\Lambda}, z\right) \\
& \quad=\epsilon\left(p_{\Xi}, p_{\Lambda}, z\right) \int_{0}^{z} \delta \mathcal{N}_{\Lambda}\left(p_{\Xi}, p_{\Lambda}, s, z\right) \\
& =\epsilon\left(p_{\Xi}, p_{\Lambda}, z\right)\left[\lambda_{\Lambda} \exp \left(-\lambda_{\Lambda} z\right) d z\right]\left[N\left(p_{\Xi}, p_{\Lambda}, 0\right) \lambda_{\Xi}\right] \int_{0}^{z} \exp \left[-\left(\lambda_{\Xi}-\lambda_{\Lambda}\right) s\right] d s \\
& \quad=\epsilon\left(p_{\Xi}, p_{\Lambda}, z\right) N\left(p_{\Xi}, p_{\Lambda}, 0\right) \frac{\lambda_{\Xi} \lambda_{\Lambda}}{\lambda_{\Xi}-\lambda_{\Lambda}}\left[\exp \left(-\lambda_{\Lambda} z\right)-\exp \left(-\lambda_{\Xi} z\right)\right] d z
\end{aligned}
$$

This distribution could then be used in $\chi^{2}$ and max-likelihood fits, where the events would be binned accordingly.

As the fit to the $\Xi^{0}$ vertex gave acceptable results, it was not necessary to fall back on the fit to the $\Lambda$ vertex distribution. In addition, because the distribution of daughter $\Lambda$ decay vertices was necessarily the result of a convolution of the $\Xi^{\mathbf{0}}$ decay vertex distribution, it was much less sensitive to the magnitude of $\tau_{\Xi^{0}}$. In fact, in this experiment, a $\Xi^{0}$ lifetime derived from a fit to the $\Lambda$ vertex distribution had an uncertainty more than twice as large as that using the $\Xi^{0}$ vertex distribution.

\subsection{Asymmetry Measurements - General}

As was discussed in Chapter 1, the angular distribution of the daughter baryon in non-leptonic hyperon decays is a function of the asymmetry parameter $(\alpha)$ and the 
polarization $(\mathcal{P})$ of the decaying baryon. In $\Lambda \rightarrow p \pi^{-}$decays, the distribution of the proton in the rest frame of the $\Lambda$ is

$$
\frac{d n(p)}{d \Omega}=\frac{n(p)}{4 \pi}\left(1+\alpha_{\Lambda} \overrightarrow{\mathcal{P}}_{\Lambda} \cdot \hat{p}\right)
$$

where $\overrightarrow{\mathcal{P}}_{\Lambda}$ is the $\Lambda$ polarization vector and $\hat{p}$ the unit vector along the proton momentum. If the $\Lambda$ 's are from the decay $\Xi^{0} \rightarrow \Lambda \pi^{0}$, then their polarization is related to the asymmetry parameters and polarization of the parent $\Xi^{0}[2]$.

$$
\overrightarrow{\mathcal{P}}_{\Lambda}=\frac{\left(\alpha_{\Xi}+\hat{\Lambda} \cdot \overrightarrow{\mathcal{P}}_{\Xi}\right) \hat{\Lambda}-\beta_{\Xi}\left(\hat{\Lambda} \times \overrightarrow{\mathcal{P}}_{\Xi}\right)-\gamma_{\Xi} \hat{\Lambda} \times\left(\hat{\Lambda} \times \overrightarrow{\mathcal{P}}_{\Xi}\right)}{1+\alpha_{\Xi} \hat{\Lambda} \cdot \overrightarrow{\mathcal{P}}_{\Xi}}
$$

The terms on the right-hand side of the equation refer to the rest frame of the $\Xi^{0}$. To use this definition of $\overrightarrow{\mathcal{P}}_{\Lambda}$ with the proton distribution from the $\Lambda$ (Equation 5.29), the lab quantities must first be Lorentz transformed to the $\Xi^{0}$ rest frame, before a subsequent Lorentz transformation to a $\Lambda$ rest frame.

As will be shown below, the proton angular distribution for $\Lambda$ 's from $\Xi^{0}$ decays was used to measure $\alpha$ and $\vec{P}_{\Xi}$ by analyzing it relative to various axes. Using such an arbitrary axis $\hat{n}$, which for each event has an angle $\theta$ relative to $\hat{p}$, the angular distribution of Equation 5.29 can be integrated about the azimuthal angle $\phi$ of $\hat{p}$ about $\hat{n}$.

$$
\frac{d n(p)}{d(\cos \theta)}=\frac{n(p)}{2}\left(1+\alpha_{\Lambda} \overrightarrow{\mathcal{P}}_{\Lambda} \cdot \hat{n} \hat{n} \cdot \hat{p}\right)
$$

The angular dependence on $\alpha_{\Lambda} \alpha_{\Xi}$, the product of the $\Lambda$ and $\Xi^{0}$ asymmetry parameters, was isolated by using the the unit vector $\hat{\Lambda}$ along the $\Lambda$ momentum (Figure 5.4) in each event as the arbitrary axis $\hat{n}$ in Equation 5.31. After substitution of the definition of $\overrightarrow{\mathcal{P}}_{\Lambda}$ from Equation 5.30 into Equation 5.31, the expression for the angular asymmetry became

$$
\frac{d n(p)}{d(\cos \theta)}=\frac{n(p)}{2}\left(1+\left[\frac{\alpha_{\Lambda} \alpha_{\Xi}+\alpha_{\Lambda} \hat{\Lambda} \cdot \overrightarrow{\mathcal{P}}_{\Xi}}{1+\alpha_{\Xi} \hat{\Lambda} \cdot \overrightarrow{\mathcal{P}}_{\Xi}}\right] \cos \theta\right)
$$

Previous measurements had established that $\alpha_{\Lambda} \alpha_{\Xi}$ was $\sim-0.25$, while $\left|\overrightarrow{\mathcal{P}}_{\Xi}\right|$ was expected to be at most 0.05 in this experiment, with its effect on the distribution reduced by 


\section{$\wedge$ Rest Frame}

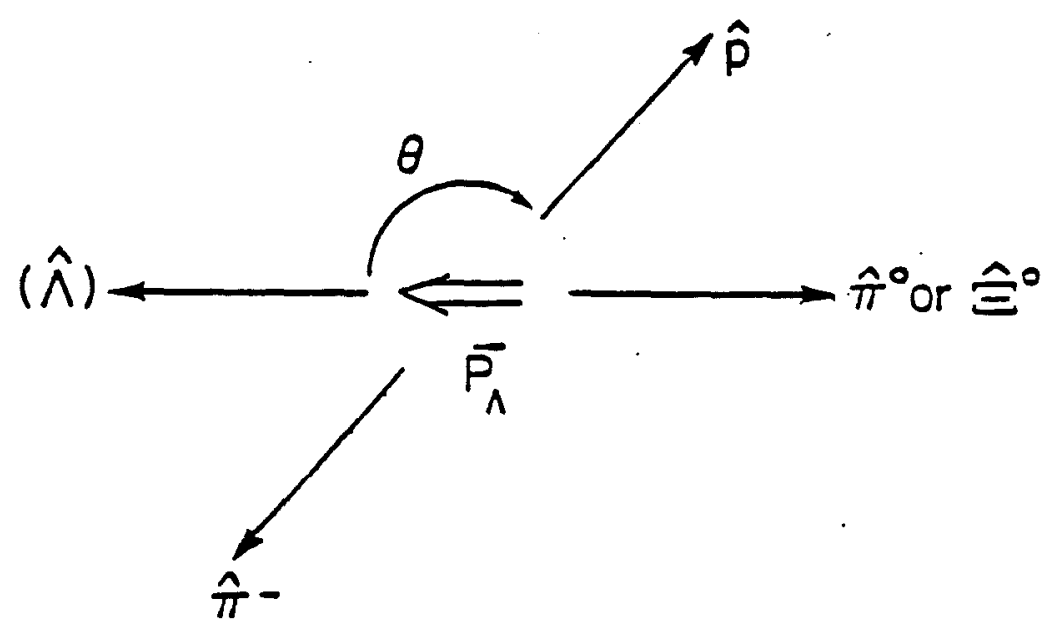

Figure 5.4: Definition of directions used in $\alpha_{\Xi}$ determination

the scalar product $\hat{\Lambda} \cdot \overrightarrow{\mathcal{P}}_{\Xi}$. This meant that the asymmetry parameters $\left(\alpha_{\Lambda} \alpha_{\Xi}\right)$ term dominated the angular distribution about $\hat{\Lambda}$. If the $\Xi^{0}$ polarization was assumed to be zero, $\left(\vec{P}_{\Xi}=0\right)$, then the angular distribution of Equation 5.32 would have a slope equal to $\alpha_{\Lambda} \alpha_{\Xi}$.

$$
\frac{d n(p)}{d(\cos \theta)}=\frac{n(p)}{2}\left(1+\alpha_{\Lambda} \alpha_{\Xi} \cos \theta\right)
$$

Any polarization $\overrightarrow{\mathcal{P}}_{\Xi}$ of the $\Xi^{0}$ particles in the experiment was relative to the fixed lab axes, $x, y$ and $z$. If the incoming proton beam in the experiment hit the target at a finite angle relative to the axis of the neutral beam collimator, then the resulting $\Xi^{0}$ 's would have a polarization $\mathcal{P}_{\Xi}$ normal to the plane defined by the incoming protons and the neutral beam. As the neutral beam was along the $z$-axis, any initial polarization was in the $x-y$ plane. Because the collimator had a vertical magnetic field, the $x$ component of the $\Xi^{0}$ polarization was precessed about the $y$-axis as it passed through the collimator, resulting in a polarization at the exit of the collimator in the $z$ direction as well.

In this experiment, while the relative angle between the incident proton beam and 
the neutral beam was small, it was not necessarily zero. The small angles involved could still have resulted in a significant transverse momentum $\left(P_{T}\right)$ of the beam particles, due to their high momentum. For instance, a $400 \mathrm{GeV}$ particle produced at an vertical angle of $1 \mathrm{mrad}$. would have a $P_{T}$ of $0.4 \mathrm{GeV}$. Previous measurements[24] had shown that this is sufficient to result in a $5 \%$ polarization. As we were at a fixed angle, the polarization would have been an increasing function of the $\Xi^{0}$ momentum.

The $\Xi^{0}$ polarization was extracted by analyzing the angular distribution of Equation 5.31 with respect each of the lab axes, i.e. $\hat{n}=$ the $x, y$ or $z$ axis. Assuming time-reversal invariance $(\beta=1)$, the expression for the distribution compared to the $x$ axis was:

$$
\left.\frac{d n(p)}{d(\cos \theta}\right)=\frac{n(p)}{2}\left(1+\alpha_{\Lambda}\left[\frac{\alpha_{\Xi} \hat{\Lambda} \cdot \hat{x}+\left(\gamma_{\Xi} \overrightarrow{\mathcal{P}}_{\Xi} \cdot \hat{x}\right)}{1+\alpha_{\Xi} \hat{\Lambda} \cdot \overrightarrow{\mathcal{P}}_{\Xi}}\right] \hat{x} \cdot \hat{p}\right)
$$

The dominant terms were due to the asymmetry parameters $\left(\alpha_{\Lambda} \alpha_{\Xi} \hat{\Lambda}\right)$ and the $\Xi^{0}$ polarization $\left(\alpha_{\Lambda} \gamma_{\Xi} \overrightarrow{\mathcal{P}}_{\Xi}\right)$. Given a value for $\alpha_{\Lambda} \alpha_{\Xi}$, then $\overrightarrow{\mathcal{P}}_{\Xi}$ could be found by fitting this distribution.

\subsubsection{Hybrid $\alpha_{\Lambda} \alpha_{\Xi}$ Fit Technique}

As was shown in Figure 4.1, the raw angular asymmetry distribution of the data did not have the expected linear dependence on $\cos \theta$ (Equation 5.33). This was because the acceptance was not uniform as a function of $\cos \theta$. The $M C$ event sample could have been used to measure this acceptance, but this would have made the $\alpha_{\Xi^{0}}$ measurement sensitive to how well the MC matched other aspects of the real events, such as the $\Xi^{0}$ lifetime. Instead, a "hybrid MC" technique was employed to correct the data for the acceptance. This method[25] had been used to analyze a series of hyperon experiments, which included a previous measurement [13] of $\alpha_{\Lambda} \alpha_{\Xi}$.

In the "hybrid MC" technique, a sample of "fake" events was generated for each real event. These fakes had all the same $\Lambda$ vertex and $\Lambda$ momenta as the real event, but had a $\cos \theta$ randomly generated from a flat distribution. Each fake event, as well 
as the real event, was required to pass selection criteria which modelled the apertures of the experimental trigger and apparatus. Only real events which passed were used in the final event sample. Sufficient numbers of possible fake events were generated for each accepted real event until 10 of them passed the selection criteria. This resulting fake event sample thus shared the same acceptance as the real data, but without being biased by $\overrightarrow{\mathcal{P}}_{\Lambda}$.

Because these fake events were not passed through the reconstruction software, the selection criteria used had to ensure that the events only fell in regions were the reconstruction efficiency was uniform and effects due to resolution were minimized. The MC event sample was very useful in determining these criteria.

Previous experience[13] with such a hybrid MC technique had shown that an imperfect matching of the acceptance and elimination of resolution effects would result in a systematic bias $\left(\vec{B}_{\Lambda}\right)$ being added to the $\Lambda$ polarization observed in the fit.

$$
\overrightarrow{\mathcal{P}}_{\Lambda}(\text { observed })=\overrightarrow{\mathcal{P}}_{\Lambda}(\text { true })+\vec{B}_{\Lambda}
$$

$\overrightarrow{\mathcal{P}}_{\Lambda}$ (true) was the $\Lambda$ polarization defined in Equation 5.30 , which was due to the $\Xi^{0}$ decay asymmetry and polarization. Because the acceptance and reconstruction were functions of the lab coordinates, such a bias would be fixed with respect to the lab coordinates. Thus, $\vec{B}_{\Lambda}$ could be separated from the $\Lambda$ polarization due to $\alpha_{\Lambda} \alpha_{\Xi}$, as $\vec{B}_{\Lambda}$ only couples indirectly through its scalar product with $\hat{\Lambda}$. It is not as clear how $\vec{B}_{\Lambda}$ could be separated from the the effects of any $\Xi^{0}$ polarization. As will be shown below, though, $\vec{B}_{\Lambda}$ was determined by splitting the data into two sets which had inherently opposite directions for $\overrightarrow{\mathcal{P}}_{\Xi}$, but not for $\vec{B}_{\Lambda}$.

The hybrid MC technique was then used to measure $\alpha_{\Lambda} \alpha_{\Xi}$ and $\overrightarrow{\mathcal{P}}_{\Xi}$. All events were summed into $20 \cos \theta$ bins, each of width 0.1 to span the range from -1 to 1 . The fake events were generated with a flat $\cos \theta$ distribution, but they were taken from real events whose distribution was affected by their asymmetry. To correct for this, each fake event $j$ generated with $\cos \theta_{i j}$ from a real event $i$ with $\cos \theta_{i}$ had to be weighted 
by a factor $W_{i j}$ :

$$
W_{i j}=\frac{1+\alpha_{\Lambda} \overrightarrow{\mathcal{P}}_{\Lambda} \cdot \hat{n} \cos \dot{\theta}_{i j}}{1+\alpha_{\Lambda} \overrightarrow{\mathcal{P}}_{\Lambda} \cdot \hat{n} \cos \theta_{i}}
$$

A $\chi^{2}$ was calculated for each $\cos \theta$ bin $I$,

$$
\chi_{I}^{2}=\frac{\left(n_{I}-n_{I}^{\prime} / N_{0}\right)^{2}}{n_{I}}
$$

where $n_{I}$ was the number of real events in $\cos \theta$ bin $I, n_{I}^{\prime}$ the weighted number of fake events, and $N_{0}$ the ratio of fake to real events $(=10)$. The number of fake events in the bin was calculated from the weights,

$$
n_{I}^{\prime}=\sum_{i j} W_{i j}(I)
$$

where the sum $j$ was only over those fake events with $\cos \theta$ within bin $I$. The overall $\chi^{2}$ was the sum of the contributions from the $20 \cos \theta$ bins. To enable the $\chi^{2}$ to be minimized as a function of the desired parameter, either $\alpha_{\Lambda} \alpha_{\Xi}$ or $\overrightarrow{\mathcal{P}}_{\Xi}$, the weights $W_{i j}$ were expanded in a Taylor series of the parameter.

\subsubsection{Initial $\alpha_{\Lambda} \alpha_{\Xi}$ Fit}

As presented above, the axis $\hat{n}$ used to extract $\alpha_{\Lambda} \alpha_{\Xi}$ was $\hat{\Lambda}$. An initial pass was made assuming $\left|\overrightarrow{\mathcal{P}}_{\Xi}\right|$ (and $\left.\left|\vec{B}_{\Lambda}\right|\right)=0$. In that case, the weights $W_{i j}$ were simply

$$
W_{i j}=\frac{1+\alpha_{\Lambda} \alpha_{\Xi} \cos \theta_{i j}}{1+\alpha_{\Lambda} \alpha_{\Xi} \cos \theta_{i}}
$$

The overall $\chi^{2}$ was then minimized as a function of $\alpha_{\Lambda} \alpha_{\Xi}$. The first few terms of the Taylor series expansion were

$$
\begin{aligned}
W_{i j}\left(\alpha_{\Lambda} \alpha_{\Xi}\right)= & 1-\left(\alpha_{\Lambda} \alpha_{\Xi}\right)\left(C_{i}-C_{i j}\right) \\
& +\left(\alpha_{\Lambda} \alpha_{\Xi}\right)^{2}\left[C_{i}\left(C_{i}-C_{i j}\right)\right]-\left(\alpha_{\Lambda} \alpha_{\Xi}\right)^{3}\left[C_{i}^{2}\left(C_{i}-C_{i j}\right)\right] \cdots
\end{aligned}
$$

Here, $C_{i}$ and $C_{i j}$ are shorthand for $\cos \theta_{i}$ and $\cos \theta_{i j}$ respectively.

Table 5.11 shows the results of this pass, broken down by $\Xi^{0}$ momentum range. The results from DE and DW targets were consistent with each other and are included 


\begin{tabular}{|c|c|r|c|c|}
\hline$P_{\Xi}(\mathrm{GeV} / \mathrm{c})$ & Avg. $P_{\Xi}$ & Events & $\alpha_{\Lambda} \alpha_{\Xi}$ & $\chi^{2}$ \\
\hline $140-180$ & 165 & 11332 & $-0.258 \pm 0.028$ & 21 \\
$180-220$ & 202 & 29087 & $-0.222 \pm 0.015$ & 26 \\
$220-260$ & 240 & 36168 & $-0.235 \pm 0.012$ & 17 \\
$260-300$ & 279 & 30750 & $-0.226 \pm 0.013$ & 25 \\
$300-340$ & 318 & 20889 & $-0.238 \pm 0.013$ & 19 \\
$340-380$ & 358 & 11559 & $-0.207 \pm 0.018$ & 25 \\
$380-420$ & 397 & 5352 & $-0.270 \pm 0.027$ & 28 \\
\hline $140-420$ & 262 & 145413 & $-0.232 \pm 0.006$ & 27 \\
\hline
\end{tabular}

Table 5.11: First pass on $\alpha_{\Lambda} \alpha_{\Xi}$ measurements, assuming $\overrightarrow{\mathcal{P}}_{\Xi}=0$. The $\chi^{2}$ is over 20 bins in $\cos \theta$

together in this sample. The average over the full momentum range was $\alpha_{\Lambda} \alpha_{\Xi}=$ $-0.232 \pm 0.006$. As is shown in Figure 5.5, no obvious momentum bias was observed. To check the technique, a MC event sample which had been generated with $\alpha_{\Lambda} \alpha_{\Xi}$ $=-0.264$ and no polarization, was analyzed with the same program. The resulting fitted $\alpha_{\Lambda} \alpha_{\Xi}$ value of $-0.268 \pm 0.006$, over the momentum range $180-380 \mathrm{GeV} / \mathrm{c}$ where statistical accuracy was good, was consistent.

\subsection{3 $\overrightarrow{\mathcal{P}}_{\Xi}$ and $\vec{B}_{\Lambda}$ Determination}

Before attempting to separate the $\overrightarrow{\mathcal{P}}_{\Xi}$ and $\vec{B}_{\Lambda}$ signals, the $\Xi^{0}$ data was analyzed for a sum of them, $\gamma_{\Xi} \overrightarrow{\mathcal{P}}_{\Xi}+\vec{B}_{\Lambda}$, to determine if there were any non-zero components. As mentioned above for this analysis, the $\hat{n}$ axis was defined to be one of the three lab axes, i.e. $\hat{\boldsymbol{x}}$. To first order in $\overrightarrow{\mathcal{P}}_{\Xi}$ and $\vec{B}_{\Lambda}$, the expression for the scalar product of $\overrightarrow{\mathcal{P}}_{\Lambda}$ and $\hat{x}$ was

$$
\begin{aligned}
\overrightarrow{\mathcal{P}}_{\Lambda} \cdot \hat{x} & =\alpha \Xi \hat{\Lambda} \cdot \hat{x}+\gamma_{\Xi} \overrightarrow{\mathcal{P}}_{\Xi} \cdot \hat{x}+\vec{B}_{\Lambda} \cdot \hat{x} \\
& =\alpha_{\Xi} \Lambda_{X}+\gamma_{\Xi} \mathcal{P}_{x}+B_{x}
\end{aligned}
$$

Here $\Lambda_{x}=\hat{\Lambda} \cdot \hat{x}, \mathcal{P}_{x}=\overrightarrow{\mathcal{P}}_{\Xi} \cdot \hat{x}$, and $B_{x}=\vec{B}_{\Lambda} \cdot \hat{x}$. In expanding the weights in a Taylor series, it was more convenient to multiply each side by $\alpha_{\Lambda}$,

$$
\alpha_{\Lambda} \overrightarrow{\mathcal{P}}_{\Lambda} \cdot \hat{x}=\left(\alpha_{\Lambda} \alpha_{\Xi}\right)_{1} \Lambda_{X}+\alpha_{\Lambda}\left[\gamma_{\Xi} \mathcal{P}_{x}+B_{x}\right]
$$




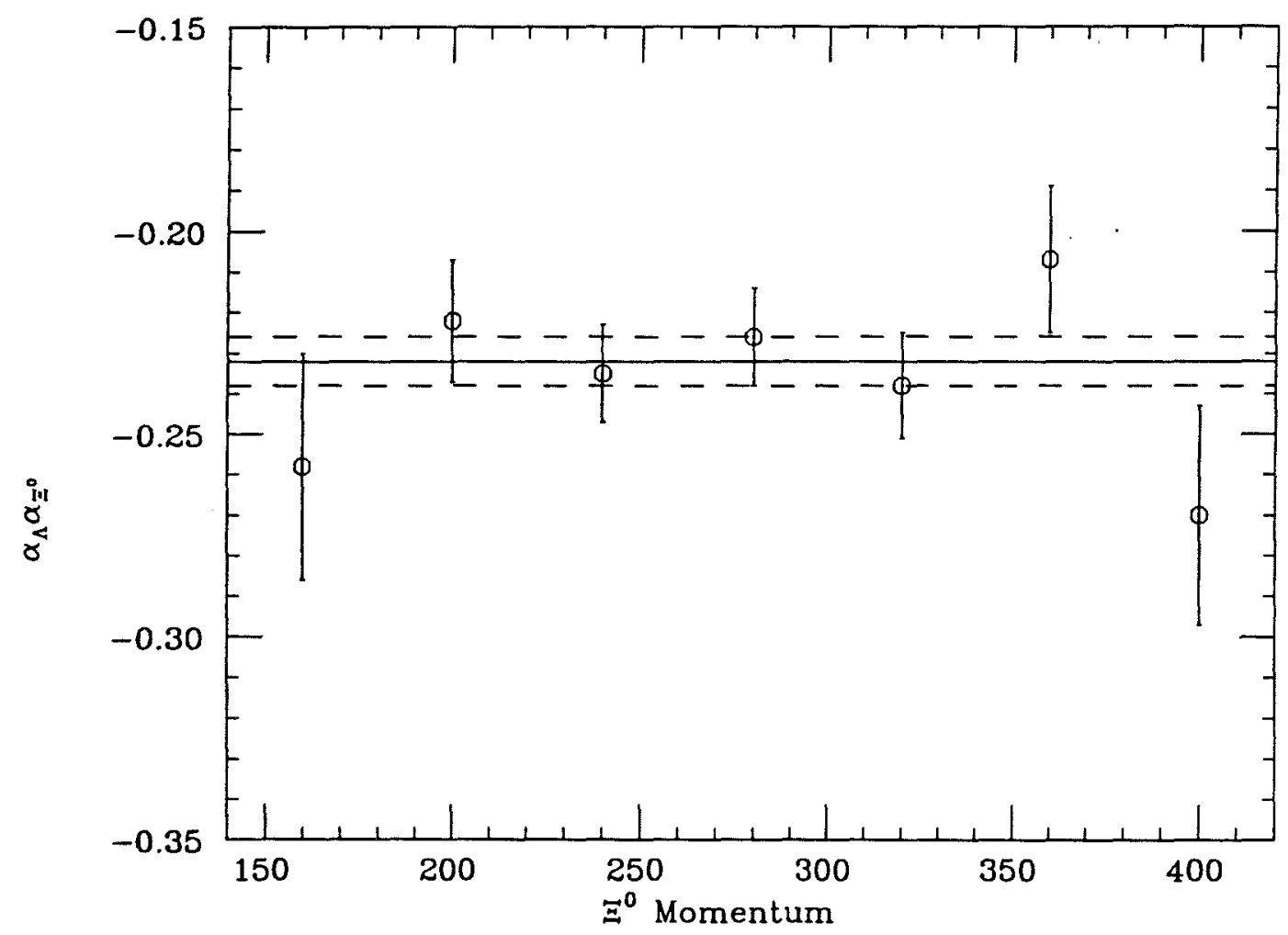

Figure 5.5: $\alpha_{\Lambda} \alpha_{\Xi}$ as a function of $\Xi^{0}$ momentum, assuming $\overrightarrow{\mathcal{P}}_{\Xi}=0$ 


\begin{tabular}{|c|cc|rc|rc|}
\hline$P_{\Xi}(\mathrm{GeV} / \mathrm{c})$ & \multicolumn{1}{c|}{$\gamma_{\Xi} \mathcal{P}_{x}+B_{x}$} & $\chi^{2}$ & \multicolumn{1}{c|}{$\gamma_{\Xi} \mathcal{P}_{y}+B_{y}$} & $\chi^{2}$ & \multicolumn{1}{c|}{$\gamma_{\Xi} \mathcal{P}_{z}+B_{z}$} & $\chi^{2}$ \\
\hline $140-180$ & $-0.003 \pm 0.038$ & 27 & $-0.016 \pm 0.034$ & 32 & $0.193 \pm 0.067$ & 19 \\
$180-220$ & $-0.034 \pm 0.019$ & 17 & $-0.053 \pm 0.019$ & 18 & $-0.141 \pm 0.037$ & 25 \\
$220-260$ & $-0.005 \pm 0.016$ & 28 & $-0.006 \pm 0.016$ & 23 & $-0.100 \pm 0.026$ & 18 \\
$\mathbf{2 6 0 - 3 0 0}$ & $-0.011 \pm 0.016$ & 12 & $0.006 \pm 0.016$ & 23 & $-0.100 \pm 0.026$ & 27 \\
$\mathbf{3 0 0 - 3 4 0}$ & $-0.055 \pm 0.017$ & 28 & $-0.036 \pm 0.020$ & 17 & $-0.161 \pm 0.028$ & 25 \\
$\mathbf{3 4 0 - 3 8 0}$ & $-0.028 \pm 0.023$ & 19 & $-0.065 \pm 0.026$ & 28 & $-0.102 \pm 0.035$ & 27 \\
$\mathbf{3 8 0 - 4 2 0}$ & $-0.055 \pm 0.034$ & 28 & $-0.040 \pm 0.039$ & 29 & $\mathbf{0 . 0 0 8} \pm 0.050$ & 35 \\
\hline $140-420$ & $-0.025 \pm \mathbf{0 . 0 0 8}$ & 45 & $-0.023 \pm 0.008$ & $\mathbf{3 1}$ & $-0.096 \pm 0.014$ & 25 \\
\hline
\end{tabular}

Table 5.12: Measurements of $\gamma_{\Xi} \mathcal{P}_{x}+B_{x}, \gamma_{\Xi} \mathcal{P}_{y}+B_{y}$ and $\gamma_{\Xi} \mathcal{P}_{z}+B_{z}$, averaged over the entire data set. The $\chi^{2}$ is again the total over $20 \cos \theta$ bins.

where $\left(\alpha_{\Lambda} \alpha_{\Xi}\right)_{1}$ was the initial value found above. The expression for the weights (Equation 5.43) was derived from this scalar product.

$$
W_{i j}=\frac{1+\left(\left(\alpha_{\Lambda} \alpha_{\Xi}\right)_{1} \Lambda_{x}+\alpha_{\Lambda}\left[\gamma_{\Xi} \mathcal{P}_{x}+B_{x}\right]\right) \cos \theta_{i j}}{1+\left(\left(\alpha_{\Lambda} \alpha_{\Xi}\right)_{1} \Lambda_{x}+\alpha_{\Lambda}\left[\gamma_{\Xi} \mathcal{P}_{x}+B_{x}\right]\right) \cos \theta_{i}}
$$

This expression was then expanded in a Taylor series of $\alpha_{\Lambda}\left[\gamma_{\Xi} \mathcal{P}_{x}+B_{x}\right]$.

$$
\begin{aligned}
W_{i j}\left(\alpha_{\Lambda}\left[\gamma_{\Xi} \mathcal{P}_{x}+B_{x}\right]\right)= & \frac{1+\left(\alpha_{\Lambda} \alpha_{\Xi}\right)_{1} \Lambda_{x} C_{i j}}{1+\left(\alpha_{\Lambda} \alpha_{\Xi}\right)_{1} \Lambda_{x} C_{i}} \\
& -\left(\alpha_{\Lambda}\left[\gamma_{\Xi} \mathcal{P}_{x}+B_{x}\right]\right) \frac{C_{i}-C_{i j}}{\left(1+\left(\alpha_{\Lambda} \alpha_{\Xi}\right)_{1} \Lambda_{x} C_{i}\right)^{2}} \\
& +\left(\alpha_{\Lambda}\left[\gamma_{\Xi} \mathcal{P}_{x}+B_{x}\right]\right)^{2} \frac{C_{i}\left(C_{i}-C_{i j}\right)}{\left(1+\left(\alpha_{\Lambda} \alpha_{\Xi}\right)_{1} \Lambda_{x} C_{i}\right)^{3}} \\
& -\left(\alpha_{\Lambda}\left[\gamma_{\Xi} \mathcal{P}_{x}+B_{x}\right]\right)^{3} \frac{C_{i}^{2}\left(C_{i}-C_{i j}\right)}{\left(1+\left(\alpha_{\Lambda} \alpha_{\Xi}\right)_{1} \Lambda_{x} C_{i}\right)^{4}} \cdots
\end{aligned}
$$

The $\chi^{2}$ (Equation 5.37) was then minimized with respect to $\alpha_{\Lambda}\left[\gamma_{\Xi} \mathcal{P}_{x}+B_{x}\right]$. The process was repeated for $\alpha_{\Lambda}\left[\gamma_{\Xi} \mathcal{P}_{y}+B_{y}\right]$ and $\alpha_{\Lambda}\left[\gamma_{\Xi} \mathcal{P}_{z}+B_{z}\right]$. The fit (Tables 5.12) was evaluated over the same momentum ranges as were used in the asymmetry measurement, and the results are plotted in Figures 5.6,5.7 and 5.8. While the $x$ and $y$ components of $\gamma_{\Xi} \overrightarrow{\mathcal{P}}_{\Xi}+\vec{B}_{\Lambda}$ were small, the z component of $\gamma_{\Xi} \overrightarrow{\mathcal{P}}_{\Xi}+\vec{B}_{\Lambda}$ was significant, averaging -0.10 .

When the MC event sample, which was created assuming no real $\Xi^{0}$ polarization, was analyzed in the same way as the real events, the resulting components of $\vec{B}_{\Lambda}$ (also 


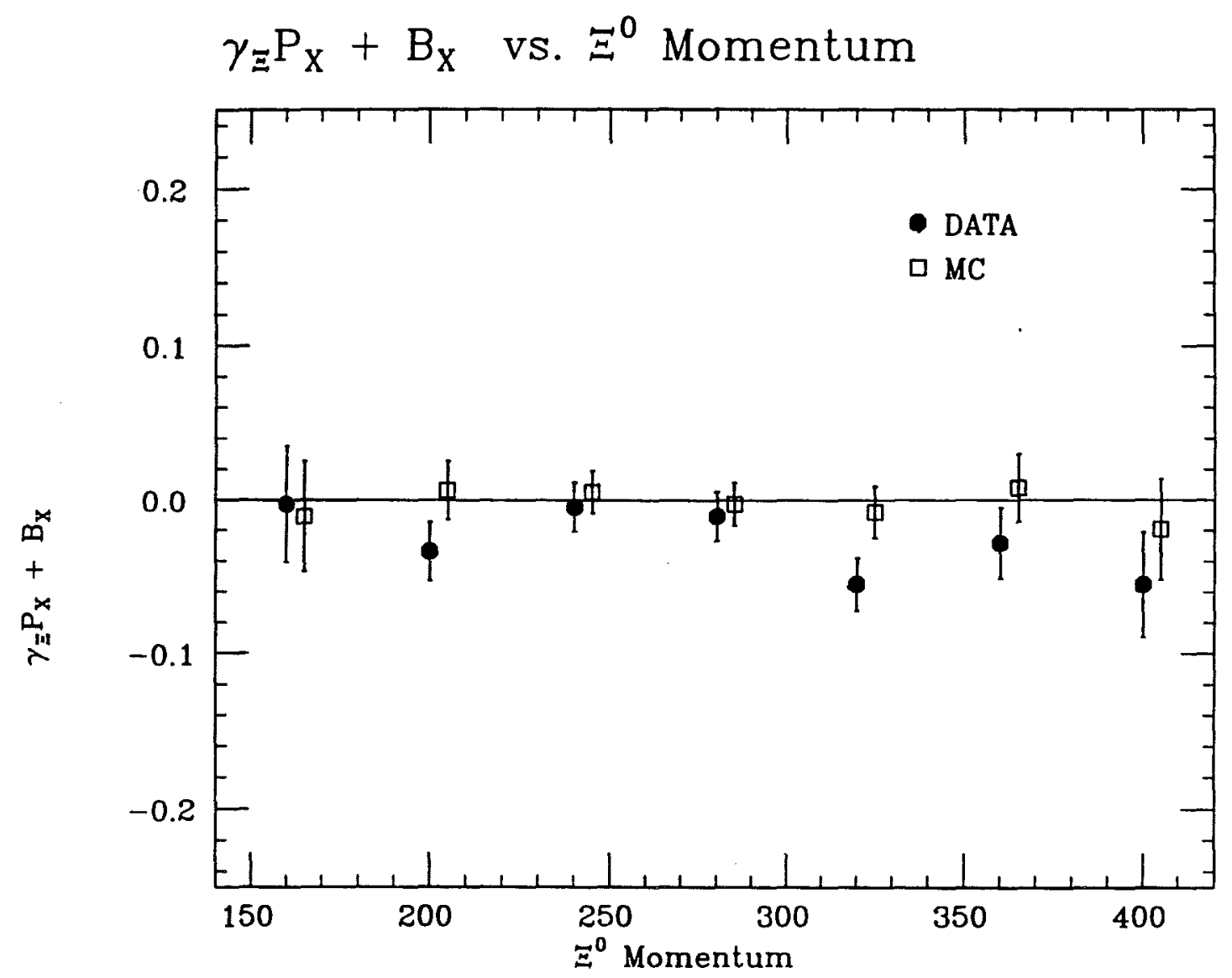

Figure 5.6: $\gamma_{\Xi} \mathcal{P}_{x}+B_{x}$ as a function of $\Xi^{0}$ momentum. Results for both data (filled circles) and MC (open squares) events are presented. 


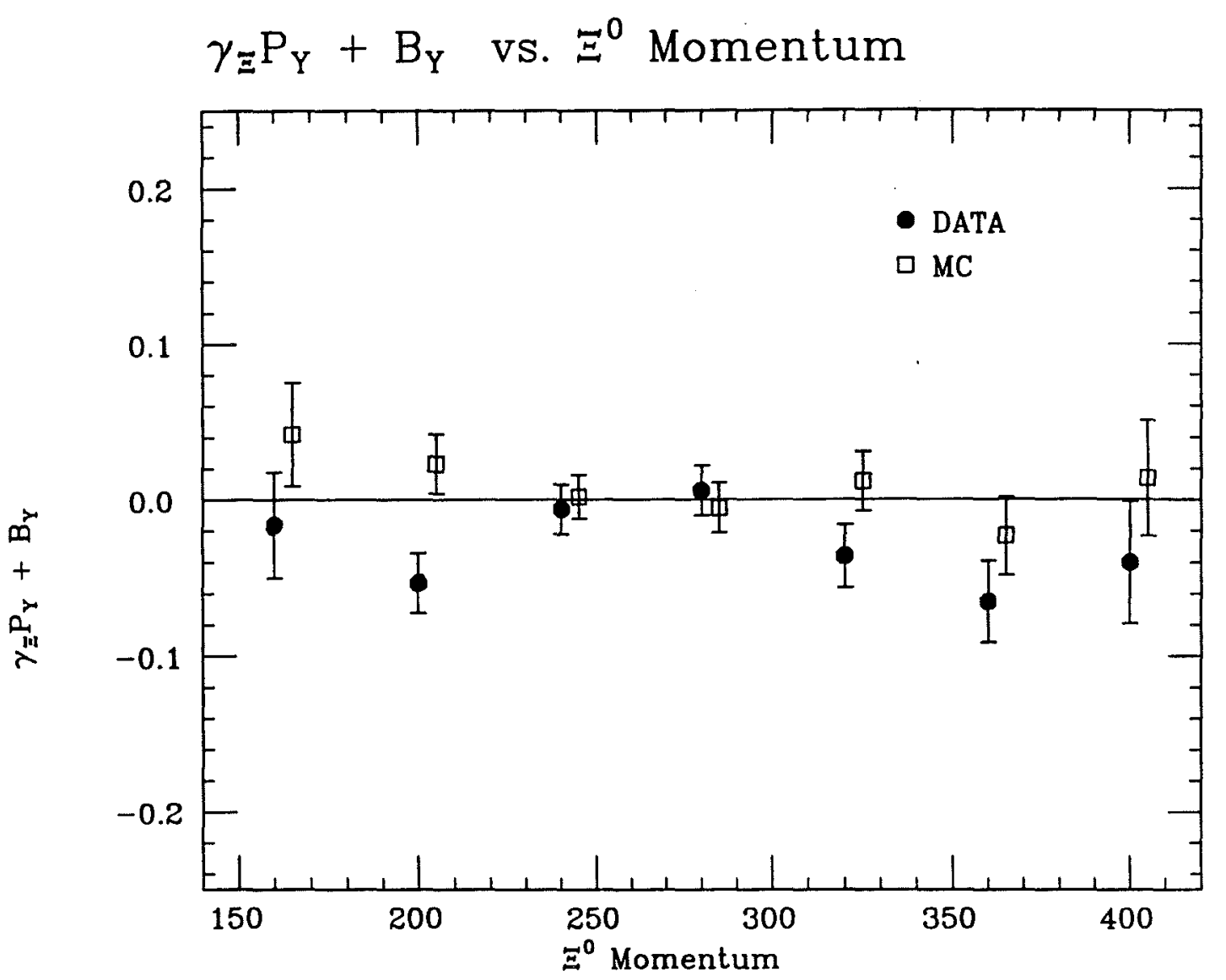

Figure 5.7: $\gamma \Xi \mathcal{P}_{y}+B_{y}$ as a function of $\Xi^{0}$ momentum. Again, results for the data (filled circles) are plotted along with those from MC events (open squares). 


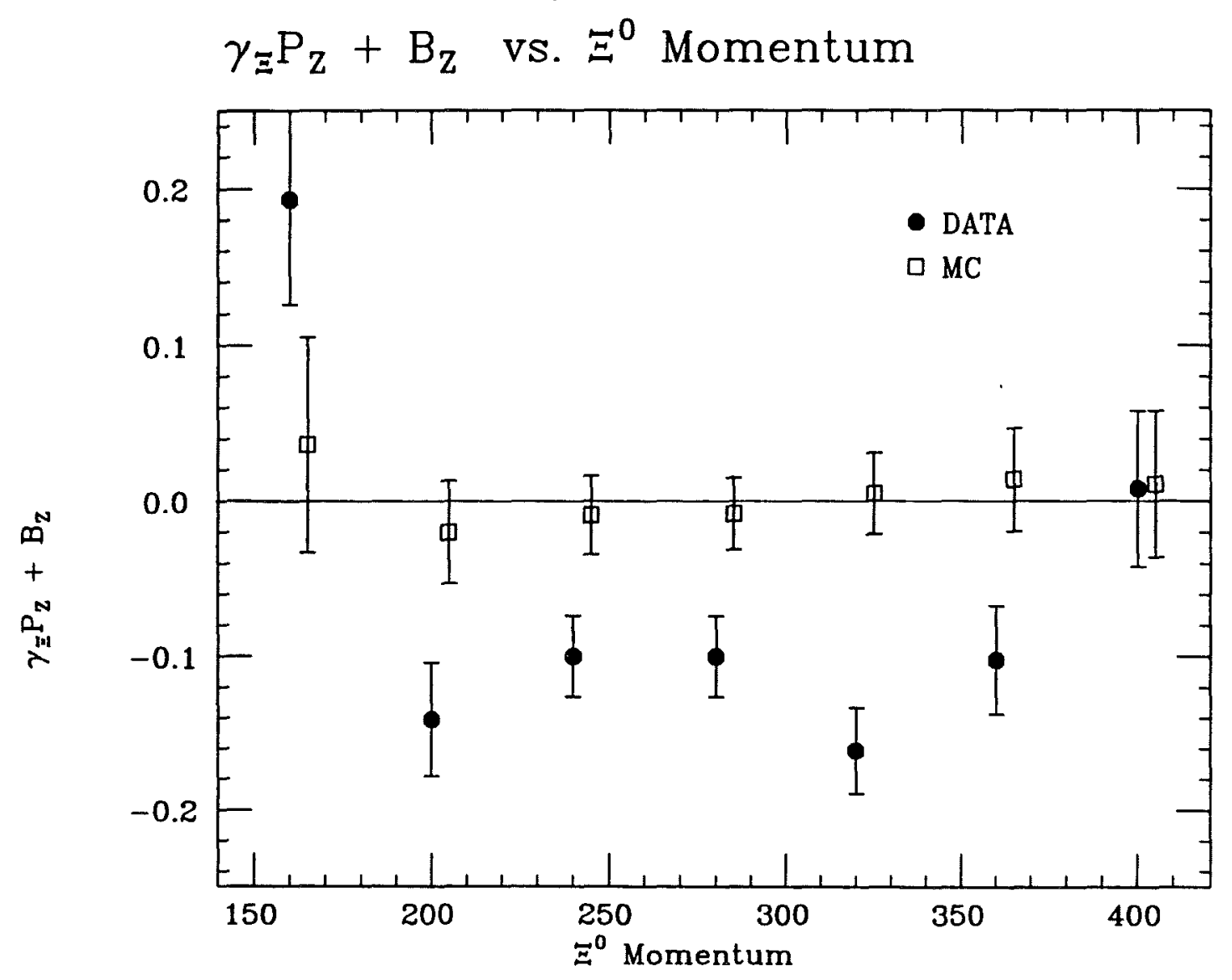

Figure 5.8: $\gamma \Xi \mathcal{P}_{z}+B_{z}$ as a function of $\Xi^{0}$ momentum. Note that the results for the data (filled circles) show a significant deviation from zero, while the MC (open squares) results were consistent with zero. 
plotted in Figures 5.6, 5.7 and 5.8) were consistent with zero. Thus, any non-zero components of $\gamma_{\Xi} \overrightarrow{\mathcal{P}}_{\Xi}+\vec{B}_{\Lambda}$ were not expected to be artifacts of the fitting procedure.

To determine whether the significant $z$ "polarization" signal was due to a true $\Xi^{0}$ polarization $\left(\overrightarrow{\mathcal{P}}_{\Xi}\right)$ in the data, or to a systematic bias $\left(\vec{B}_{\Lambda}\right)$, the signal's dependence on the polarity of the field of the sweeper magnet was analyzed. As was mentioned above, any $\Xi^{0}$ polarization in the $z$ direction could only arise due to the precession of an initial $x$ polarization of the $\Xi^{0}$ beam in the magnetic field of the collimator. The precession angle $\Phi$ which any $\overrightarrow{\mathcal{P}}_{\Xi}$ would have been rotated through in the $\mathbf{x}-\mathbf{z}$ plane was a function of the integral magnetic field $(B \cdot d \ell)$ and the magnetic moment $\mu_{\Xi}$ :

$$
\Phi=-18.30 \mu \Xi \int B \cdot d \ell
$$

Here $\Phi$ is in degrees, while $\mu_{\Xi}$ is in nuclear magnetons, and $B \cdot d \ell$ in Tesla-meters. In this experiment, $\Phi=528$ degrees, which would have rotated $\mathcal{P}_{x}$ so that at the exit of the collimator there were both $\mathrm{x}$ and $\mathrm{z}$ components. The direction of the precession, and hence the sign of $\mathcal{P}_{z}$, changed with the polarity of the magnetic field. On the other hand, any bias $\left(B_{z}\right)$ would be independent of the sweeper field polarity, which had no effect on the apparatus. The lack of any significant $\gamma_{\Xi} \mathcal{P}_{x}+B_{x}$ signal in the data indicated that the $\gamma_{\Xi} \mathcal{P}_{z}+B_{z}$ signal should be predominantly due to bias. During the experiment, this sweeper magnet polarity was changed on a regular basis (Table 2.3).

The $\Xi^{0}$ data was split into two roughly equal sets according to the polarity of the sweeper magnet during each run. $\gamma_{\Xi} \mathcal{P}_{z}+B_{z}$ was then measured separately for each set, resulting in $\gamma_{\Xi} \mathcal{P}_{z}+B_{z}(+)$ from the positive-polarity runs, and $\gamma_{\Xi} \mathcal{P}_{z}+B_{z}(-)$ from the negative-polarity runs. The $\gamma_{\Xi} \mathcal{P}_{z}+B_{z}$ signals from each set could then be combined in different ways to determine the $z$ component of $\overrightarrow{\mathcal{P}}_{\Xi}$ and $\vec{B}_{\Lambda}$. The difference in $\gamma_{\Xi} \mathcal{P}_{z}+B_{z}(+)$ and $\gamma_{\Xi} \mathcal{P}_{z}+B_{z}(-)$ provided the $z$ component of $\overrightarrow{\mathcal{P}}_{\Xi}$.

$$
\gamma_{\Xi} \mathcal{P}_{z}=\frac{\left[\gamma_{\Xi} \mathcal{P}_{z}+B_{z}\right](+)-\left[\gamma_{\Xi} \mathcal{P}_{z}+B_{z}\right](-)}{2}
$$




\begin{tabular}{|c|c|c|c|c|}
\hline$P_{\Xi}(\mathrm{GeV})$ & $\mathrm{DE} \gamma_{\Xi} \mathcal{P}_{z}$ & $\mathrm{DE} B_{z}$ & $\mathrm{DW} \gamma_{\Xi} \mathcal{P}_{z}$ & $\mathrm{DW} B_{z}$ \\
\hline $140-180$ & $-0.034 \pm 0.100$ & $0.090 \pm 0.100$ & $0.030 \pm 0.084$ & $0.229 \pm 0.084$ \\
\hline $180-220$ & $0.003 \pm 0.055$ & $-0.145 \pm 0.055$ & $-0.025 \pm 0.051$ & $-0.137 \pm 0.051$ \\
\hline $220-260$ & $0.020 \pm 0.039$ & $-0.050 \pm 0.039$ & $-0.019 \pm 0.037$ & $-0.094 \pm 0.037$ \\
\hline $260-300$ & $0.039 \pm 0.037$ & $-0.092 \pm 0.037$ & $-0.016 \pm 0.036$ & $-0.151 \pm 0.036$ \\
\hline $300-340$ & $0.016 \pm 0.040$ & $-0.136 \pm 0.040$ & $-0.044 \pm \mathbf{0 . 0 3 9}$ & $-0.170 \pm 0.039$ \\
\hline $340-380$ & $-0.107 \pm 0.051$ & $-0.128 \pm 0.051$ & $-0.017 \pm 0.048$ & $-0.051 \pm 0.048$ \\
\hline $380-420$ & $0.226 \pm 0.075$ & $0.083 \pm 0.075$ & $0.131 \pm 0.070$ & $-0.033 \pm 0.070$ \\
\hline $140-420$ & $0.020 \pm 0.020$ & $-0.083 \pm 0.020$ & $-0.014 \pm 0.020$ & $-0.083 \pm 0.020$ \\
\hline
\end{tabular}

Table 5.13: $\gamma_{\Xi} \mathcal{P}_{z}$ and $B_{z}$ for $\mathrm{DE}$ and DW target event samples. These results show that the $\Xi^{0}$ data was consistent with no polarization along $z$.

The average of $\gamma_{\Xi} \mathcal{P}_{z}+B_{z}(+)$ and $\gamma_{\Xi} \mathcal{P}_{z}+B_{z}(-)$ provided the $z$ component of $\vec{B}_{\Lambda}$.

$$
B_{z}=\frac{\left[\gamma_{\Xi} \mathcal{P}_{z}+B_{z}\right](+)+\left[\gamma_{\Xi} \mathcal{P}_{z}+B_{z}\right](-)}{2}
$$

As the DE and DW target events might have had different polarizations, the samples were evaluated separately, with the results presented in Table 5.13. It was clear from these results that the $z$ component of $\gamma_{\Xi} \overrightarrow{\mathcal{P}}_{\Xi}+\vec{B}_{\Lambda}$ was not consistent with any polarization of the $\Xi^{0}$ beam, but had to be due to the bias, which was consistent between the DE and DW event samples. It was then also assumed that the small $x$ and $z$ components were also associated with the bias.

\subsubsection{Final $\alpha_{\Lambda} \alpha_{\Xi}$ Fit}

The measured biases (Table 5.12) were then used in a second iteration of the $\alpha_{\Lambda} \alpha_{\Xi}$ measurement. The expression for $\overrightarrow{\mathcal{P}}_{\Lambda} \cdot \hat{\Lambda}$ was expanded to

$$
\overrightarrow{\mathcal{P}}_{\Lambda} \cdot \hat{\Lambda}=\alpha_{\Xi}+\vec{B}_{\Lambda} \cdot \hat{\Lambda}
$$

using the measured $\left|\overrightarrow{\mathcal{P}}_{\Xi}\right|$. This was inserted into the expression for the weights (Equation 5.36), which was again expanded in a Taylor series in $\alpha_{\Lambda} \alpha_{\Xi}$.

$$
\begin{aligned}
& W_{i j}\left(\alpha_{\Lambda} \alpha_{\Xi}\right)= \\
& \quad \frac{1+\alpha_{\Lambda} \vec{B}_{\Lambda} \cdot \hat{\Lambda} C_{i j}}{1+\alpha_{\Lambda} \vec{B}_{\Lambda} \cdot \hat{\Lambda} C_{i}}-\left(\alpha_{\Lambda} \alpha_{\Xi}\right) \frac{\left(C_{i}-C_{i j}\right)}{\left(1+\alpha_{\Lambda} \vec{B}_{\Lambda} \cdot \hat{\Lambda} C_{i}\right)^{2}}
\end{aligned}
$$




\begin{tabular}{|c|c|c|}
\hline$P_{\Xi}(\mathrm{GeV})$ & $\alpha_{\Lambda} \alpha_{\Xi}$ & $\chi^{2}$ \\
\hline $140-180$ & $-0.195 \pm 0.028$ & 20 \\
$180-220$ & $-0.257 \pm 0.015$ & 25 \\
$220-260$ & $-0.248 \pm 0.012$ & 16 \\
$260-300$ & $-0.236 \pm 0.012$ & 21 \\
$300-340$ & $-0.245 \pm 0.013$ & 19 \\
$340-380$ & $-0.205 \pm 0.018$ & 22 \\
$380-420$ & $-0.268 \pm 0.027$ & 27 \\
\hline $140-420$ & $-0.242 \pm 0.006$ & 27 \\
\hline
\end{tabular}

Table 5.14: Final $\alpha_{\Lambda} \alpha_{\Xi}$ measurements. The $\chi^{2}$ is for $20 \cos \theta$ bins.

$$
+\left(\alpha_{\Lambda} \alpha_{\Xi}\right)^{2} \frac{C_{i}\left(C_{i}-C_{i j}\right)}{\left(1+\alpha_{\Lambda} \vec{B}_{\Lambda} \cdot \hat{\Lambda} C_{i}\right)^{3}}-\left(\alpha_{\Lambda} \alpha_{\Xi}\right)^{3} \frac{C_{i}^{2}\left(C_{i}-C_{i j}\right)}{\left(1+\alpha_{\Lambda} \vec{B}_{\Lambda} \cdot \hat{\Lambda} C_{i}\right)^{4}} \cdots
$$

The results of this second $\alpha_{\Lambda} \alpha_{\Xi}$ analysis are in Table 5.14. If the first and last momentum bins were dropped, the average of $\alpha_{\Lambda} \alpha_{\Xi}(-0.242 \pm 0.006)$ was the same as for the full momentum range. Figure 5.9 shows that there was no significant momentumdependent behavior in the asymmetry measurement. This new value of $\alpha_{\Lambda} \alpha_{\Xi}$ was then used in an iteration of the fit for $\vec{B}_{\Lambda}$. The results were nearly identical to the first pass results (Table 5.12) on the bias, indicating that the fits had converged.

The systematic error due to the bias was estimated by varying the values of the biases which were input to the asymmetry fits within the measured uncertainties. The systematic error due to this was $\mathbf{0 . 0 0 4}$. Other systematic uncertainties added another 0.002 to the dominant systematic due to the bias correction. Thus the final average using the hybrid method was

$$
\alpha_{\Lambda} \alpha_{\Xi}=-0.242 \pm 0.006 \text { (statistical) } \pm 0.006(\text { systematic })
$$

Using the the existing world average for $\alpha_{\Lambda}$ of $0.642 \pm 0.013$, the value of $\alpha_{\Xi^{0}}$ derived from the measured $\alpha_{\Lambda} \alpha_{\Xi}$ was $\alpha_{\Xi^{0}}=-0.377 \pm 0.011 \pm 0.009$. 


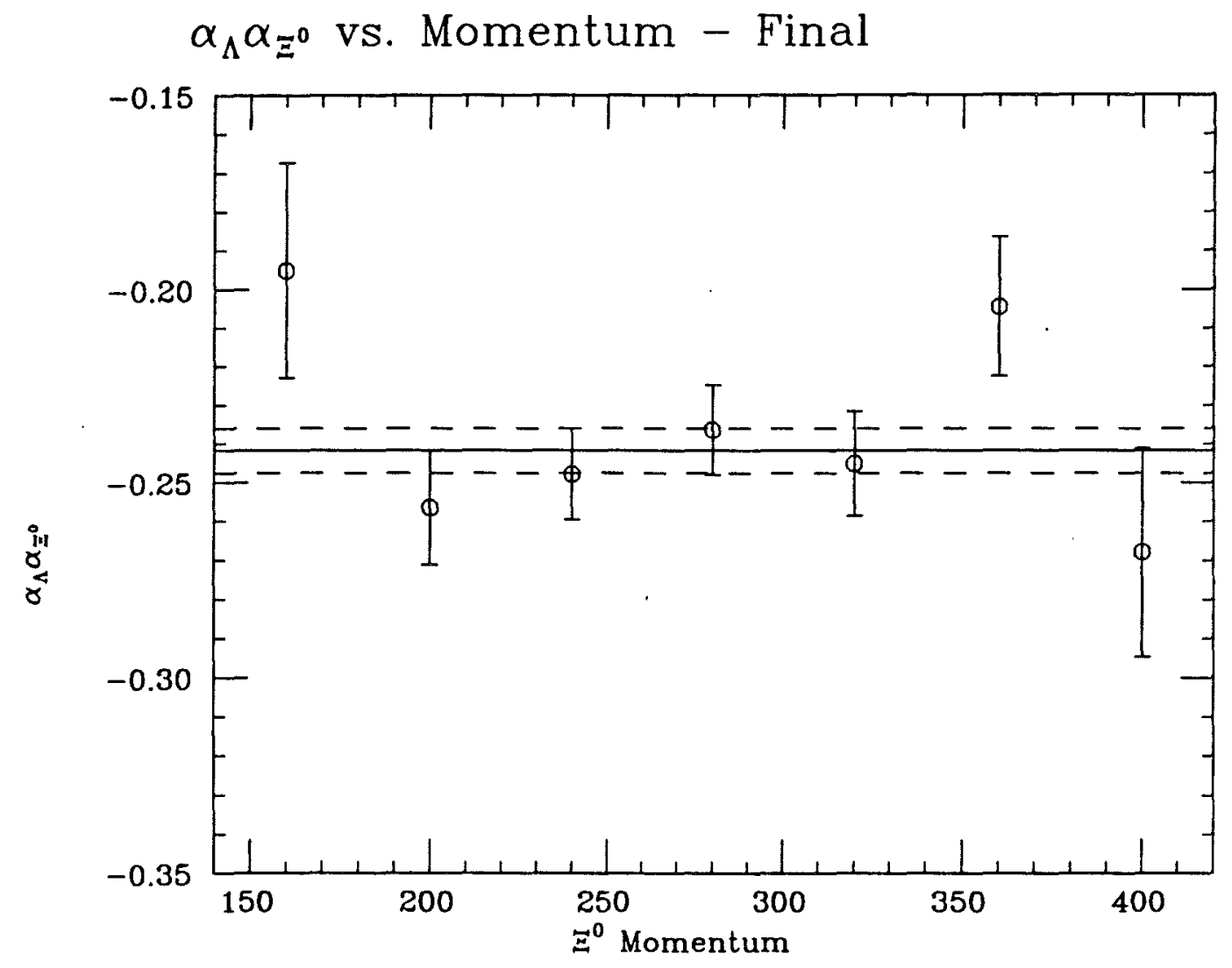

Figure 5.9: $\alpha_{\Lambda} \alpha_{\Xi}$ as a function of $\Xi^{0}$ momentum. The solid and dashed lines indicate the average $\alpha_{\Lambda} \alpha_{\Xi}$ of $-0.242 \pm 0.006$. 


\section{Chapter 6}

\section{Conclusions}

\section{1 $\Lambda$ Lifetime}

The measured value for $\tau_{\Lambda}$ from this experiment was $2.62 \pm 0.01 \times 10^{-10} \mathrm{~s}$, where the error was a combination of statistical and systematic uncertainties. As shown in Figure 6.1, our result was consistent with the other high-statistics measurements $[26,27,15]$ used to calculate the existing world average for $\tau_{\Lambda}$ of $2.631 \pm 0.020 \times 10^{-10} \mathrm{~s}$. When this new result was taken in a weighted average with the others, the new average value was $2.624 \pm 0.008 \times 10^{-10} \mathrm{~s}$. The total $\chi^{2}$ of the four measurements used, compared to the new average, was 5.0. This indicated that the variation in the measurements was not totally due to statistical fluctuations. To take account of this variation in the measurements, the Particle Data Group[3] scales the uncertainty in the world average by a scale factor $\mathrm{SF}$ :

$$
\mathrm{SF}=\sqrt{\chi^{2} /(N-1)}
$$

where $N$ is the number of measurements. In this case, the scale factor was 1.3 . Thus, the new world average would be

$$
\tau_{\Lambda}=2.624 \pm 0.010 \times 10^{-10} \mathrm{~s}
$$




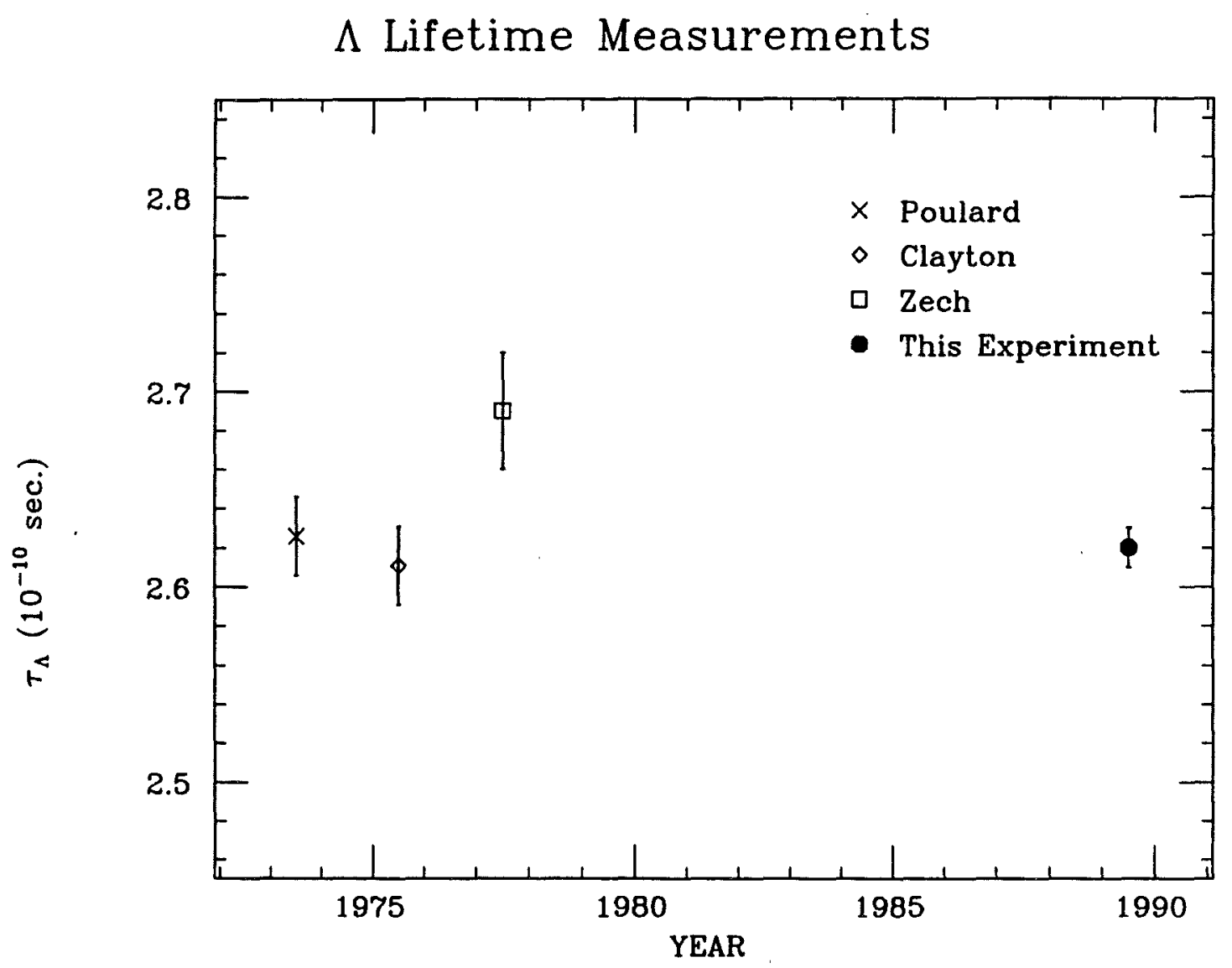

Figure 6.1: Comparision of $\tau_{\Lambda}$ result with other existing measurements $[26,27,15]$. 


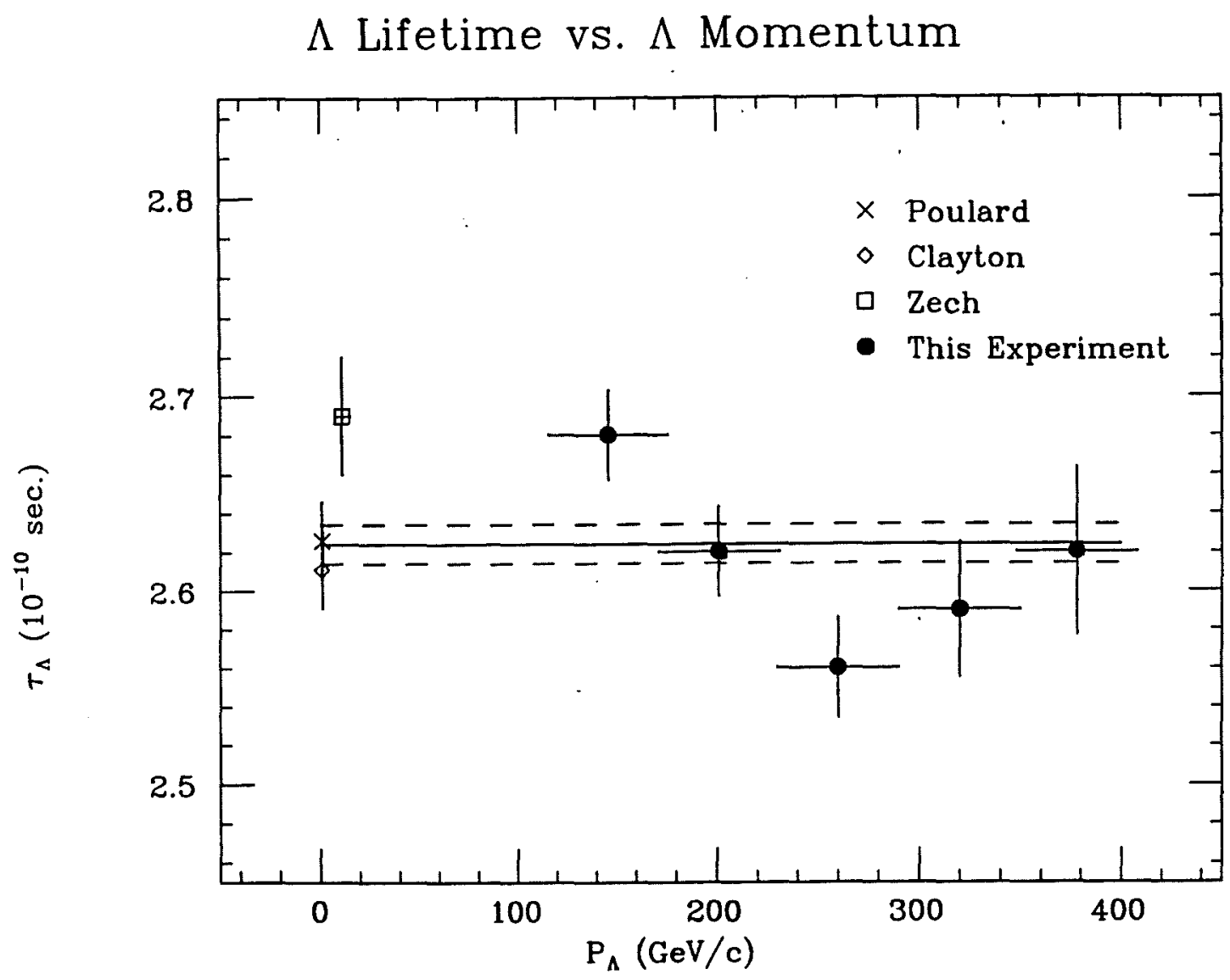

Figure 6.2: Measurements of $\tau_{\Lambda}$ as a function of $\Lambda$ momentum. The solid and dashed lines indicate the new world average of $2.624 \pm 0.010 \times 10^{-10} \mathrm{~s}$. The lack of variation with momentum is a strong confirmation of Lorentz invariance.

The previous measurements were all taken at low $\Lambda$ momentum $(<20 \mathrm{GeV} / \mathrm{c})$. If the data from this experiment (Table 5.7) and that from the previous measurements is plotted as a function of the $\Lambda$ momentum (Figure 6.2), no momentum dependence was observed. The stability of the $\Lambda$ lifetime from 1 to $400 \mathrm{GeV} / \mathrm{c}$ is a strong confirmation of its Lorentz invariance.

\section{$6.2 \Xi^{0}$ Lifetime}

The measured value for $\tau_{\Xi^{0}}$ from this experiment was $2.98 \pm 0.04$ (stat.) \pm 0.02 (syst.) $\times$ $10^{-10} \mathrm{~s}$. When the statistical and systematic errors were taken in quadrature, the result 


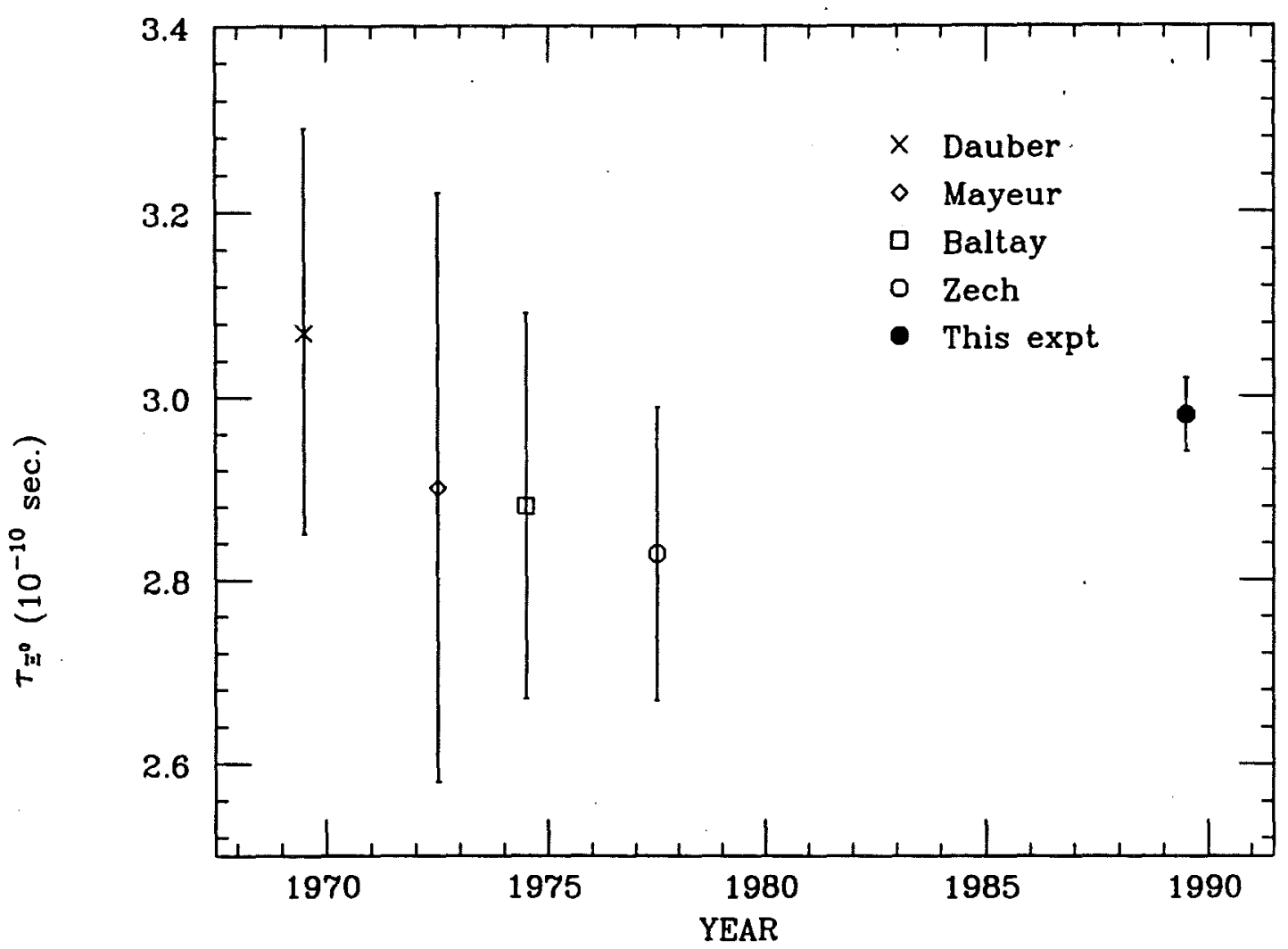

Figure 6.3: Comparision of $\tau_{\Xi^{0}}$ result with other existing measurements $[28,29,30,15]$.

was:

$$
\tau_{\Xi^{0}}=2.98 \pm 0.04 \times 10^{-10} \mathrm{~s}
$$

As shown in Figure 6.3, this new measurement was consistent with the other existing measurements $[28,29,30,15]$, as well as with the existing world average of $2.90 \pm 0.10 \times$ $10^{-10} \mathrm{~s}$. Combining the new result with the others gave a new world average of

$$
\tau_{\Xi^{0}}=2.97 \pm 0.04 \times 10^{-10} \mathrm{~s}
$$

As with the $\Lambda$ data, the previous measurements were all taken at low momentum $(<20 \mathrm{GeV} / \mathrm{c}$ ). If the data from this experiment (Table 5.9) and that from the previous measurements is plotted as a function of the $\Xi^{0}$ momentum (Figure 6.4), no momentum dependence was observed. The stability of the $\Xi^{0}$ lifetime from 1 to $380 \mathrm{GeV} / \mathrm{c}$ is 
$\Xi^{0}$ Lifetime vs. $\Xi^{0}$ Momentum

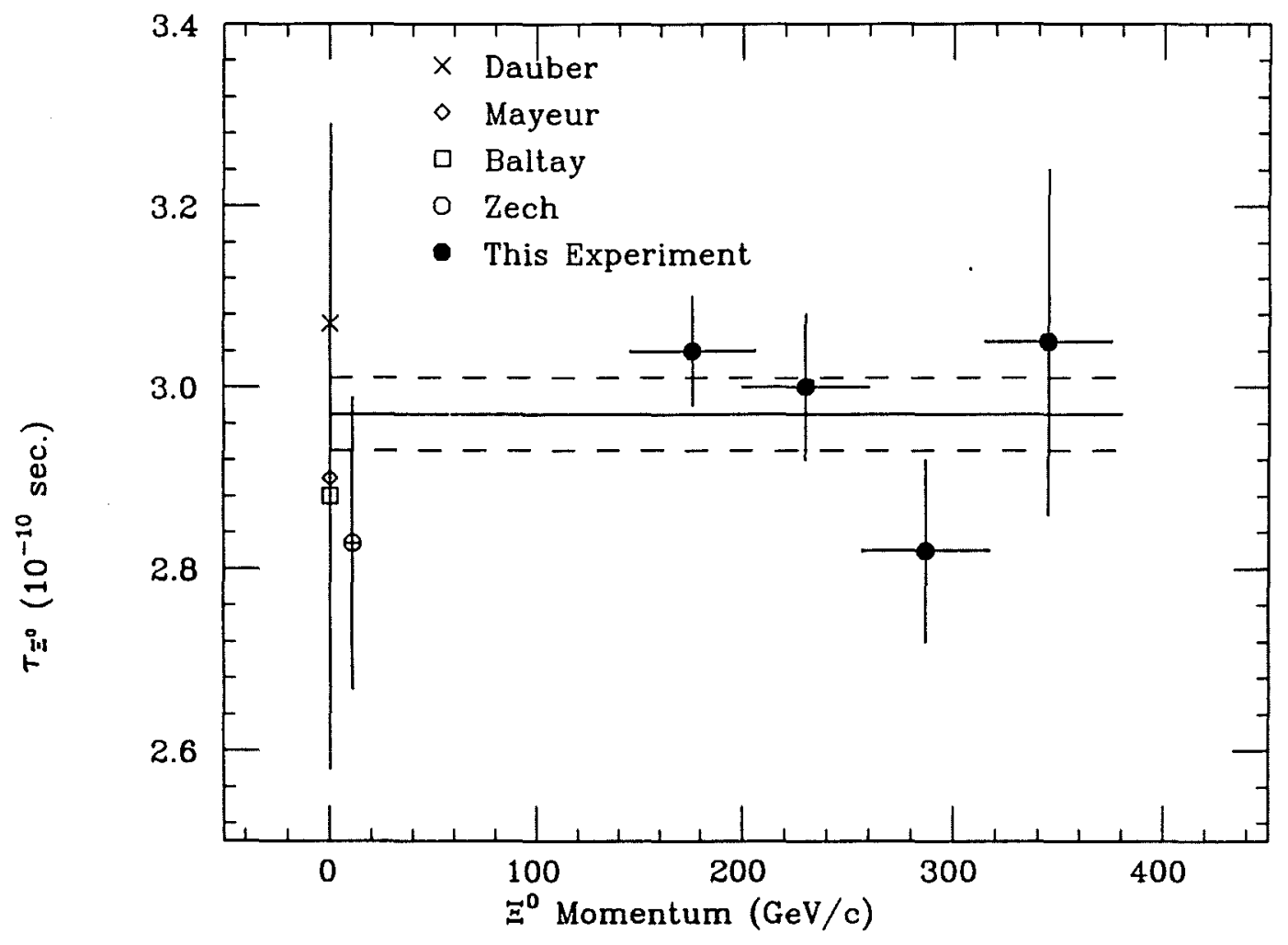

Figure 6.4: Measurements of $\tau_{\Xi^{0}}$ as a function of $\Xi^{0}$ momentum. The solid and dashed lines indicate the new world average of $2.97 \pm 0.04 \times 10^{-10} \mathrm{~s}$. Again, the lack of variation with momentum is consistent with Lorentz invariance. 


\section{$\alpha_{\Lambda} \alpha_{\Xi^{0}}$ Measurements}

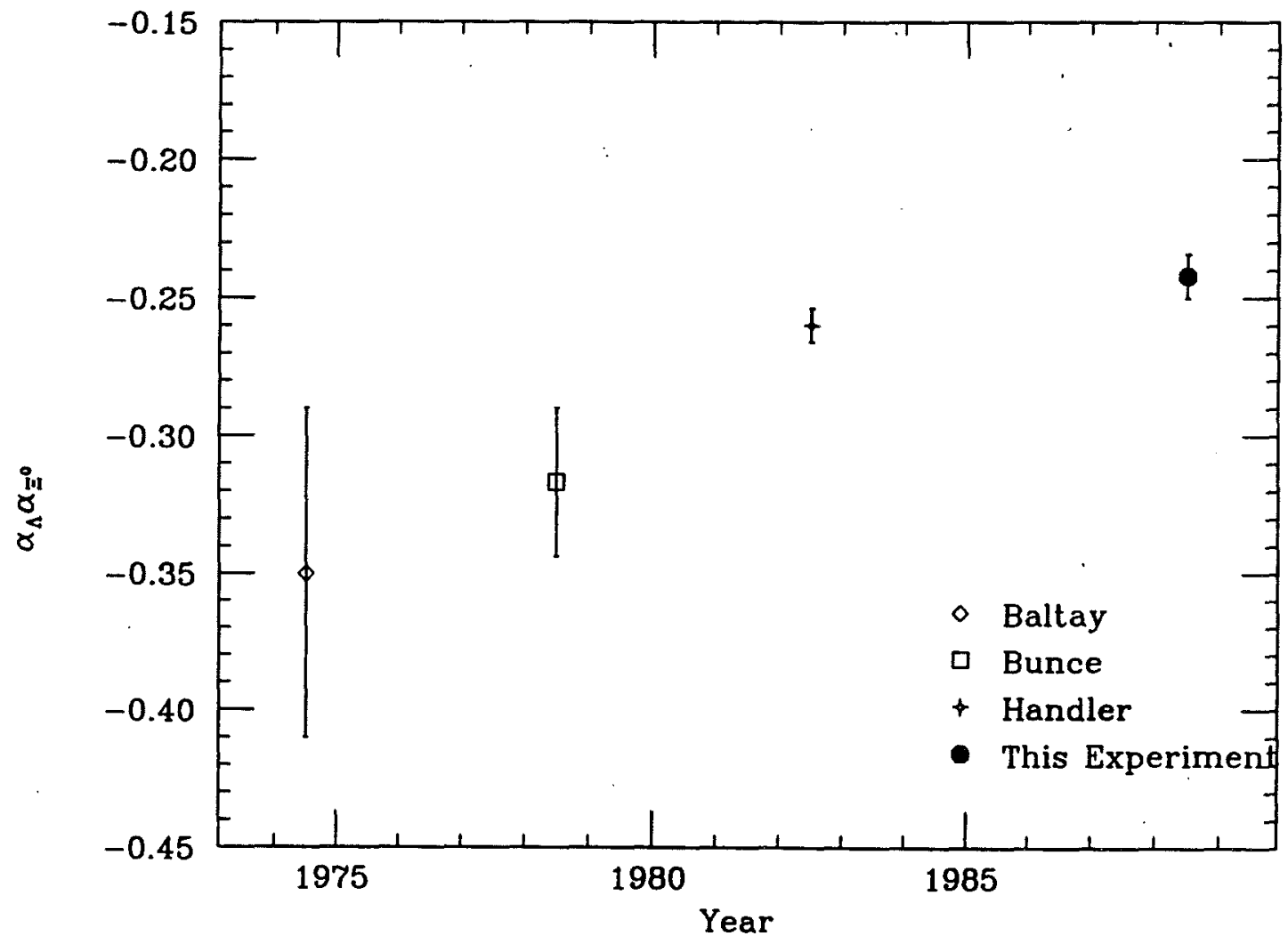

Figure 6.5: Comparision of $\alpha_{\Lambda} \alpha_{\Xi}$ result with other existing measurements[13,31].

consistent with Lorentz invariance.

\section{3 $\quad \Xi^{0}$ Asymmetry}

The value for $\alpha_{\Lambda} \alpha_{\Xi}$ from this experiment was $-0.242 \pm 0.006$ (stat.) \pm 0.006 (syst.). When the statistical and systematic errors were taken in quadrature, the total uncertainty was 0.008. As shown in Figure 6.5, the $\alpha_{\Lambda} \alpha_{\Xi}$ result from this experiment was moderately consistent with the two most recent existing measurements[13,31], differing by about two standard deviations. The new world average, using this new result and the other existing measurements, was $-0.256 \pm 0.006$ with a combined $\chi^{2}$ of 11.2 over 5 points. The resulting scale factor of 1.68 was used to increase the uncertainty, giving a final 
world average of

$$
\alpha_{\Lambda} \alpha_{\Xi}=-0.256 \pm 0: 008
$$

Using the world average for the value of $\alpha_{\Lambda}, 0.642 \pm 0.013$, to evaluate the value of $\alpha_{\Xi^{0}}$, the new world average was

$$
\alpha_{\Xi^{0}}=-0.399 \pm 0.015
$$

This was the first time that both the $\Xi^{0}$ lifetime and decay asymmetry had been measured in the same experiment. This meant that the resulting measurements would minimize any systematic difficulties when comparing the results of different experiments.

\subsection{Theoretical Implications}

The new world averages for the $\Xi^{0}$ lifetime and decay asymmetry imply new values for the $S$ and $P$-wave decay amplitudes (See Section 1.1). The new value for the $S$ wave amplitude, $A$, is $1.54 \pm 0.01$, which reduces the uncertainty a factor of 3 . The new value of $B$, the $P$ wave amplitude, is $5.30 \pm 0.21$, compared to the previous value of $5.53 \pm 0.23$. While these are not dramatic improvements, they do lead to the overall refinement of the theoretical predictions.

A summary of the new measurements as well as the old and new world averages is presented in Table 6.1. 


\begin{tabular}{|l|c|r|r|c|}
\hline Quantity & Units & Old World Average & This Experiment & New Average \\
\hline$\tau_{\Lambda}$ & $10^{-10} \mathrm{~s}$ & $2.631 \pm 0.020$ & $2.62 \pm 0.01$ & $2.624 \pm 0.008$ \\
$\tau_{\Xi^{0}}$ & $10^{-10} \mathrm{~s}$ & $2.90 \pm 0.10$ & $2.98 \pm 0.04 \pm 0.02$ & $2.97 \pm 0.04$ \\
$\alpha_{\Lambda} \alpha_{\Xi}$ & - & $-0.264 \pm 0.008$ & $-0.242 \pm 0.006 \pm 0.006$ & $-0.256 \pm 0.008$ \\
& & $( \pm 0.013)$ & & \\
$\alpha_{\Xi^{0}}$ & - & $-0.411 \pm 0.015$ & & $-0.399 \pm 0.015$ \\
& & $( \pm 0.022)$ & & \\
$A\left(\Xi^{0}\right)$ & - & $1.55 \pm 0.03$ & & $1.54 \pm 0.01$ \\
$B\left(\Xi^{0}\right)$ & - & $5.53 \pm 0.23$ & & $5.30 \pm 0.21$ \\
& & $( \pm 0.32)$ & & \\
\hline
\end{tabular}

Table 6.1: Summary of experimental results and old and new world averages. The old world averages were taken from the Particle Data Group summary of 1988[3]. The errors in brackets for $\alpha_{\Lambda} \alpha_{\Xi}, \alpha_{\Xi^{0}}$ and $B\left(\Xi^{0}\right)$ reflect the published uncertainties, which appear to over-estimate the uncertainty in $\alpha_{\Lambda} \alpha_{\Xi}$ by using a scaling factor of 2.1. This value is the square of the quantity (1.46) that one calculates using the PDG formula for the scale factor(Equation 6.1). The unbracketed errors are those found using this reduced scale factor of 1.46 . 


\section{Appendix A}

\section{$\gamma$ Trigger Electronics}

The electronics to implement the photon trigger described in Chapter 2 were designed and built by the High Energy Physics Group at the University of Minnesota. The $\gamma$ trigger electronics had to be fast enough to determine the number of clusters within $100 \mathrm{~ns}$ of receiving the signals from the lead glass blocks and the $\pi$-killer veto counters. Because the rates of lead glass signals during the run was expected to be high ( > $1 \mathrm{MHz} / \mathrm{block}$ in the center of the array), the trigger needed to have very little or no dead-time. Due the constraints of time and budget, the electronics also had to be simple and relatively inexpensive.

A block diagram of the trigger is shown in Figure A.1 The trigger electronics were spread over five modules, which were installed in a CAMAC electronics crate. This crate provided the power for the trigger and housed the discriminators for the lead glass and $\pi$-killer signals. The two Veto Boards used the horizontal and vertical $\pi$-killer counter signals to veto the appropriate lead glass signals before passing them on to the Matrix Boards. These Matrix Boards implemented the three cluster-edge counting algorithms in parallel circuits. Each of the three algorithm circuits output a signal whose level was proportional to the number of clusters. The Summing Output Board summed the outputs from each Matrix Board and used discriminators to provide output logic signals 


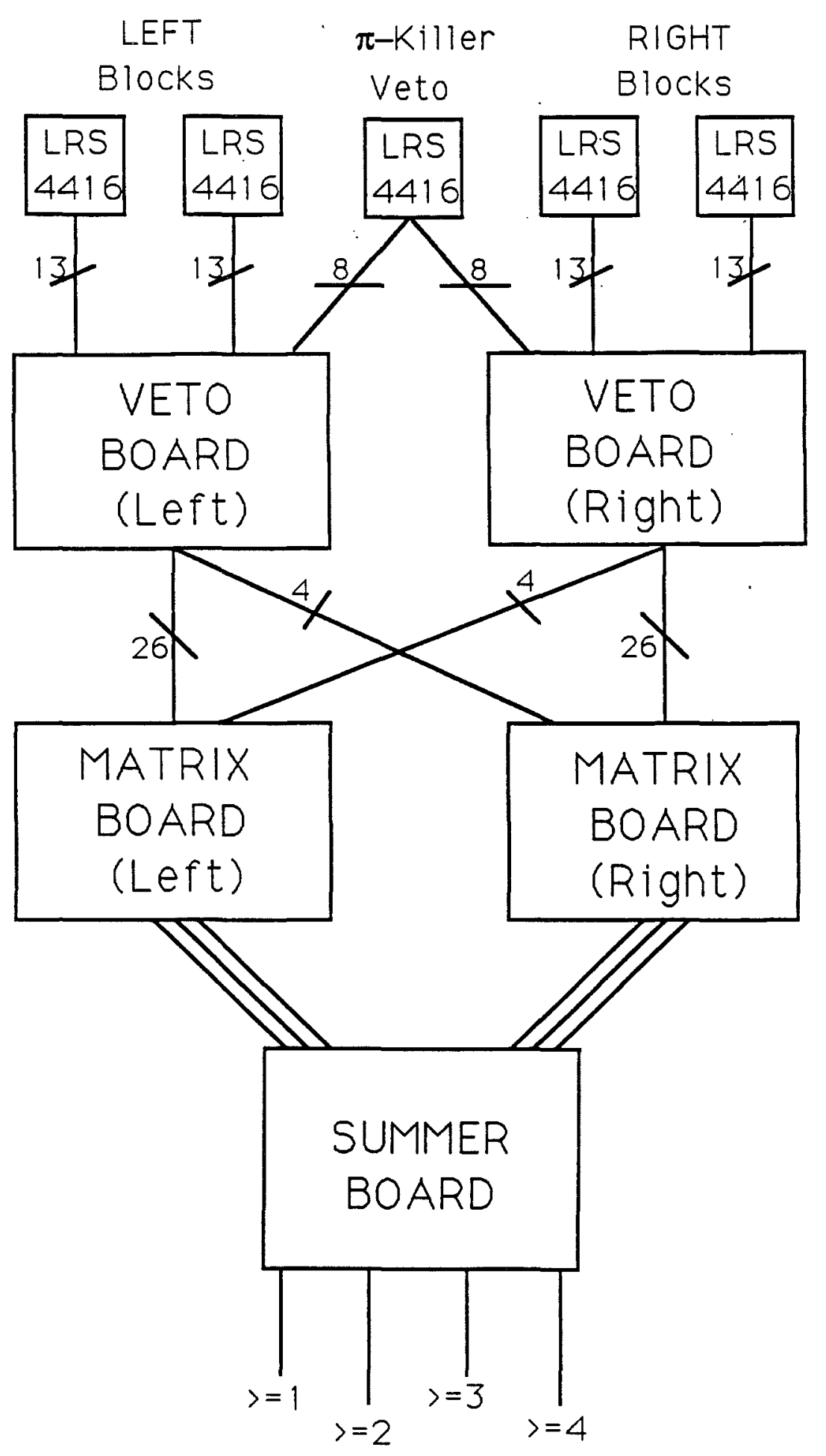

Figure A.1: Block Diagram of the $\gamma$ Trigger Electronics 
corresponding to $\geq 1, \geq 2, \geq 3$, and $\geq 4$ clusters in the array.

Most of the electronics were implemented with Advanced Transistor-Transistor Logic (TTL) circuits. The inputs were Emitter-Coupled Logic (ECL) signals to be compatible with the discriminator outputs, and the outputs were NIM level logic signals.

\section{A.1 Discriminators}

The signals from the photo-multipliers attached to the $\pi$-killer veto counters, as well as those from the lead glass blocks, were carried on coaxial cables into LeCroy Research System (LRS) Model 4416 Discriminators. These CAMAC modules had 16 channels each, with bipolar ECL outputs. The $16 \pi$-killer inputs, 6 from the horizontal elements and 10 from the vertical elements (See Figure 2.6), went to one LRS 4416. The output widths for the $\pi$-killer discriminator were set at $60 \mathrm{~ns}$. For the lead glass blocks, only the signals from the 52 blocks not on the outside of the array (Figure A.2) were used in the trigger. To take advantage of the bi-lateral symmetry of the lead glass array, these 52 blocks were separated into matching left and right halves of 26 blocks each. The output of the $\pi$-killer discriminator was similarly split into two halves of 8 counters each. The blocks were connected to 4 LRS 4416's, 13 blocks per discriminator. The lead glass block discriminators had an output width of $20 \mathrm{~ns}$ each. This allowed for a 20 ns cushion on either side of a coincidence between a lead glass block and corresponding veto signal from the $\pi$-killer.

\section{A.2 Veto Boards}

The outputs of the discriminators went to two Veto Boards (Figure A.3). Due to the symmetry of the array, the left and right Veto, and subsequent Matrix, Boards were nearly identical. The $\pi$-killer Overlap Circuit (Figure A.4) on the Veto Boards formed coincidences between overlapping horizontal and vertical $\pi$-killer counters, and 


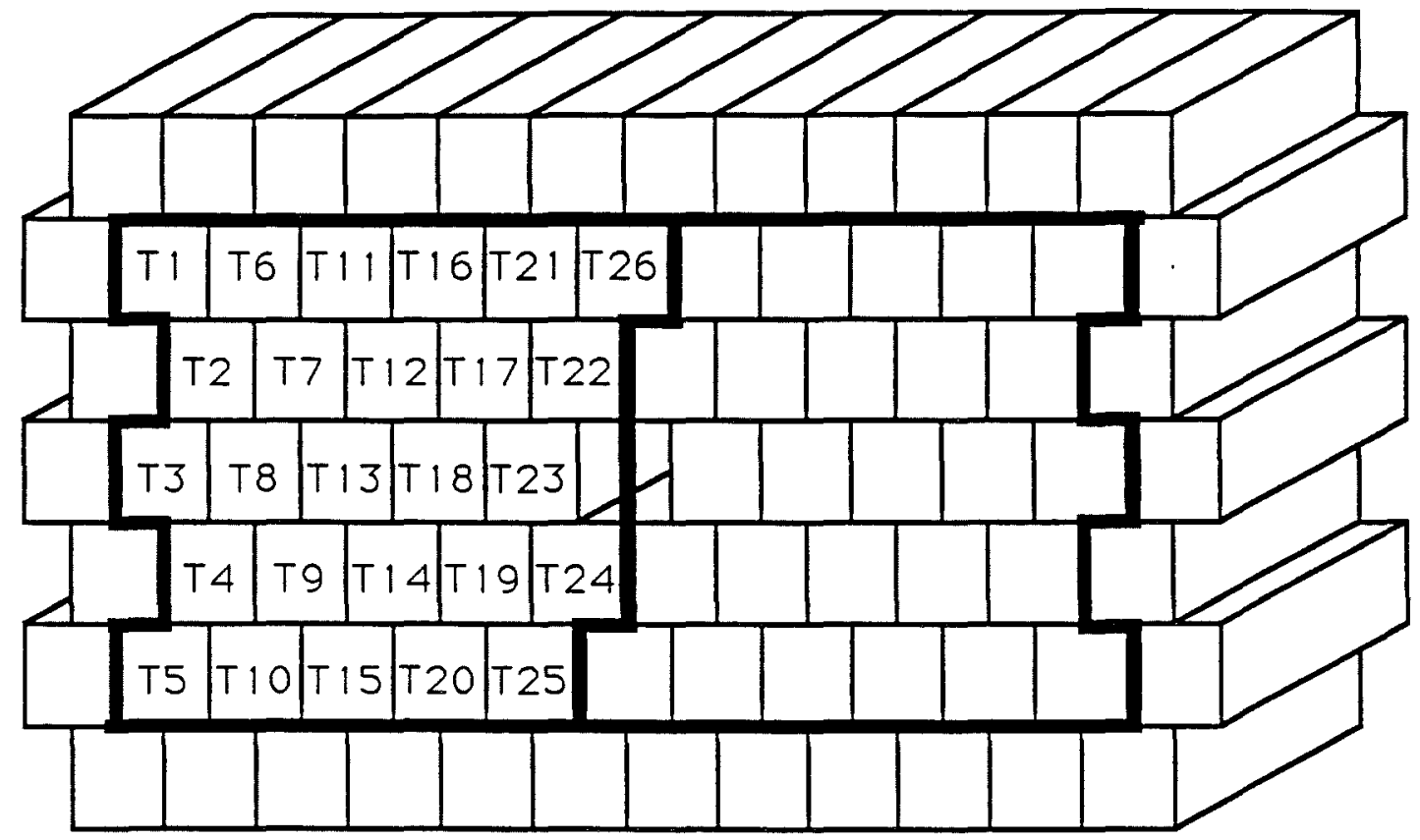

Figure A.2: Lead glass array blocks used in the $\gamma$ trigger. Note that the 52 inner blocks were split into two matching halves of 26 blocks each. 


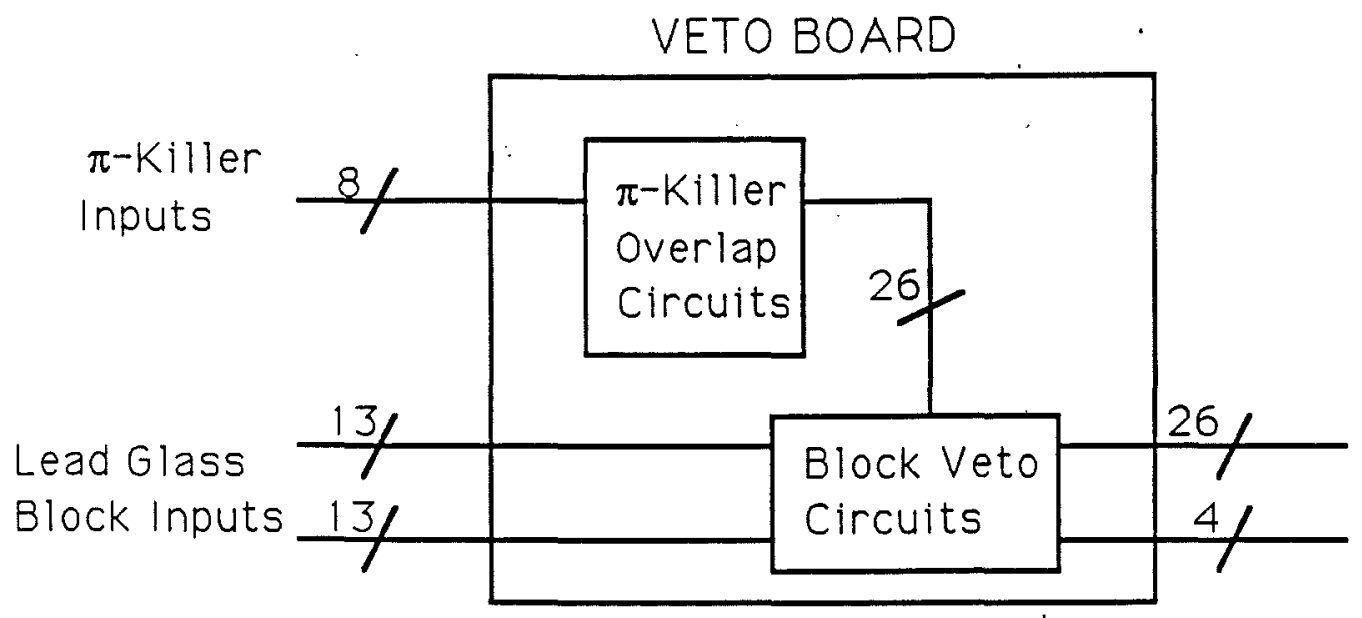

Figure A.3: Block diagram of $\gamma$ trigger Veto Board.

generated veto outputs for up to eight blocks in the trigger adjacent to the overlap of the $\pi$-killer counters (See Figure 2.12). The results of a coincidence between $\pi$-killer counters $H V 2$ and $V V 3$ are highlighted in in Figure A.4. These block vetoes were then combined with the 26 lead glass block inputs in the Block Veto circuits (Figure A.5). If there were a signal from a given lead glass block, and no corresponding $\pi$-killer block veto, then the output from the NOR gate (74S27) was a TTL HIGH level for that block, otherwise it was a LOW. The following driver (74ALS244) boosted the signals for fanout to the Matrix Boards. To simplify checking that each of the blocks had the same relative timing at the input to the trigger, a test point which was the sum of all 26 block inputs was provided. Another test point which was the sum of the $8 \pi$-killer inputs was also provided on the board.

\section{A.3 Matrix Boards}

The outputs of the Veto Boards were the inputs to the two Matrix Boards. Each Matrix Board contained the edge-algorithm circuitry for the 26 blocks on either the left or right 

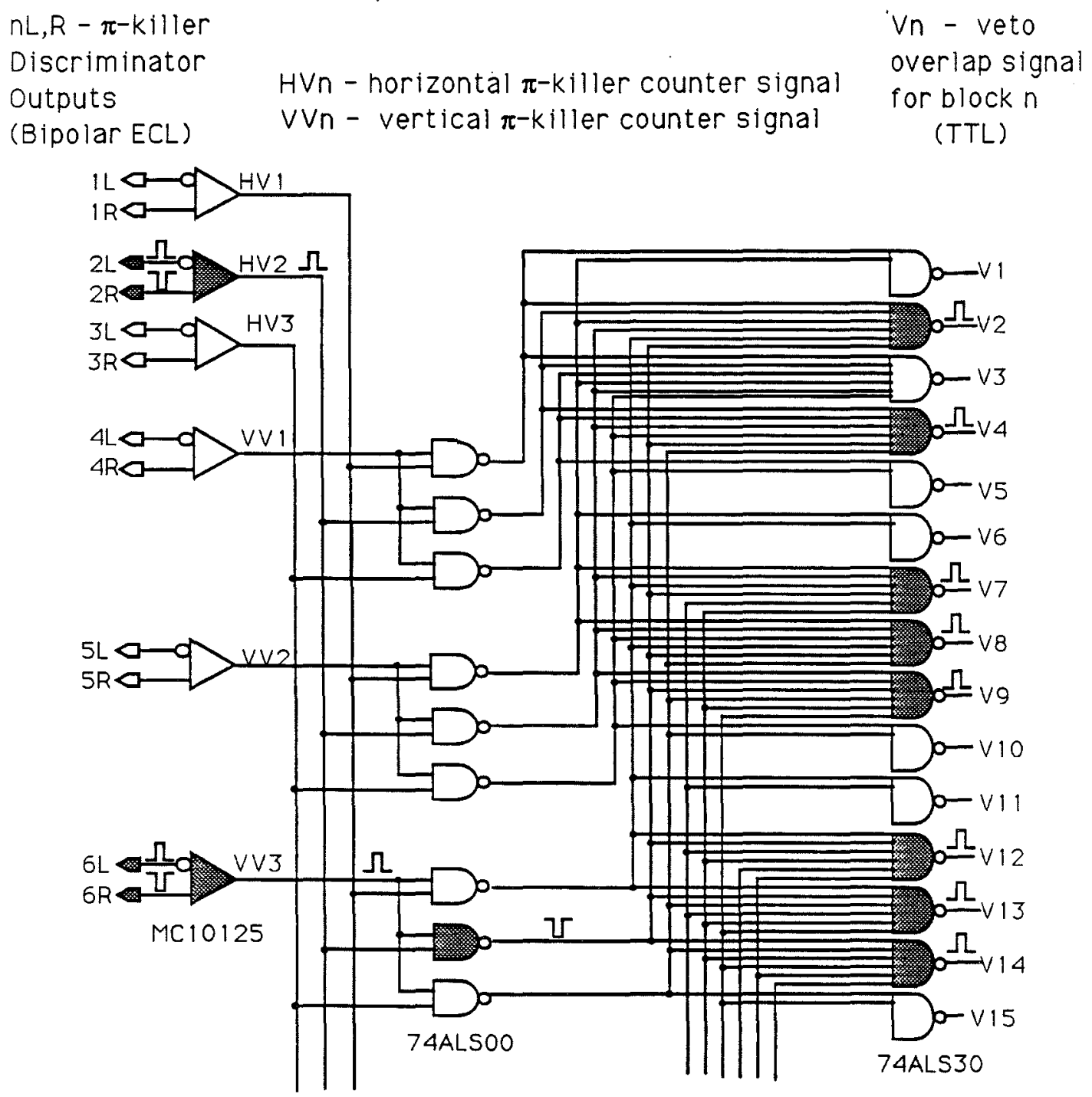

Figure A.4: $\pi$-killer Overlap Circuit on the Veto Boards. $H V 1-H V 3$ were the horizontal $\pi$-killer counter inputs, while $V V 1-V V 5$ were the vertical $\pi$-killer counter inputs. Only a portion of the full circuit is shown. The cross-hatching highlights which blocks were vetoed by a coincidence of hits in the $H V 2$ and $V V 3$ counters. $V 1-V 26$ were the veto outputs corresponding to blocks $T 1-T 26$. 


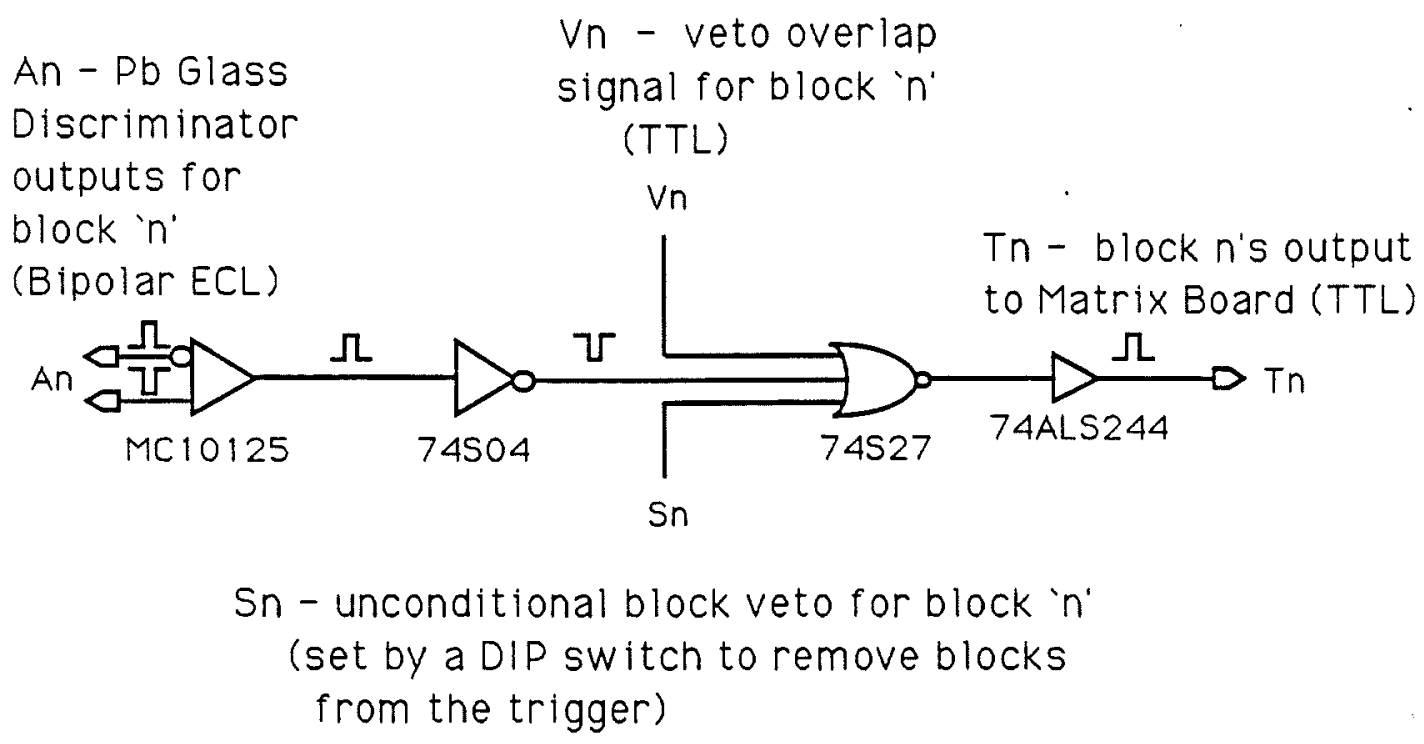

Figure A.5: Block Veto circuit on the Veto Boards. This is one of 26 such circuits on each board. The $\pi$-killer block veto for block ' $n$ ' $(V n)$ and lead glass block signal $(A n)$ were combined to provide the outputs $(T n)$ to be sent to the Matrix Boards. A block could be removed from the trigger by connecting switch input $S n$ to ground. 
side of the array. Thus, most of their inputs were only from the corresponding Veto Board for that side. To complete the algorithms for the. 4 blocks on the boundary between the two sides, there were 4 inputs from the other Veto Board. The Matrix Boards consisted of 26 identical unit cell circuits, which were only differentiated by the particular blocks hooked to the inputs of each circuit (Figure A.6).

The input to each circuit was a TTL driver (74ALS244). This driver fanned out the incoming block signal to an inverter (74S04), and a pulse-shaping Schottky inverter $(7414)+$ AND gate (74S08) circuit. The inverted block signal was then fanned out to be used as a nearest-neighbor input to other unit cells. The Schottky inverter had an inherent pulse delay of 15-22 ns. The logical AND of this delayed inverted pulse and the original pulse resulted in the AND gate ouput pulse width being limited to the same 15-22 ns. The output of the AND was fanned out to the three edgecounting circuits for that block. A four-input AND (74H21) was used to implement each edge algorithm, combining the block signal and the inverted signals from three nearest neighbors. Nearest-neighbor inputs from blocks which were not in the trigger were tied to $+5 \mathrm{~V}$, so they would be interpreted as a logical 'TRUE'. A 'TRUE' output (a TTL High) from the AND gate switched on a transistor (2N5770). The emitters of all the transistors for one of the three edge algorithms (MV1,2 or 3) were connected together to form a "wire OR" which would have its current increased by a fixed amount for each AND gate which was HIGH. A $1 \%$ tolerance $1 \mathrm{k} \Omega$ biasing resistor was used to keep the current contributions from each of the 26 unit cell relatively consistent.

\section{A.4 Summing Output Board}

The three summed outputs (MV1,2 and 3) from each of the two Matrix Boards were the inputs to the Summing Output Board (Figure A.7). The two inputs (Left and Right) from the same edge-counting algorithm (MV1 in the figure) were first summed on the Output Summing Board, and then passed to four discrimination circuits (AM287 / 
Block $X$ and its Nearest-Neighbors $A-F$ :
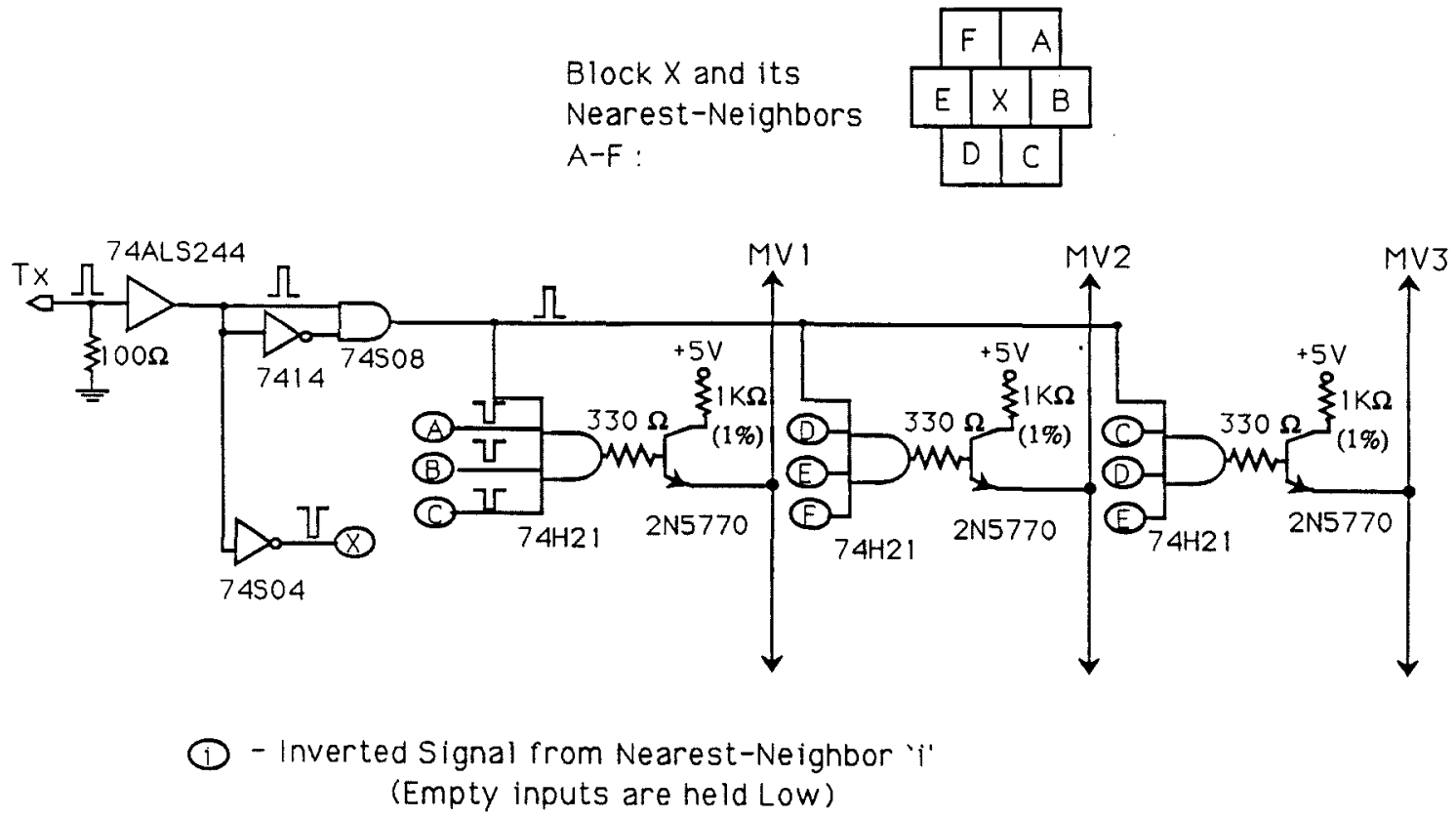

MV1,2,3 - Wire-OR Sum Outputs from the 26 Matrix Unit Cells For Edge Algorithms 1,2,3

Figure A.6: Unit cell for a block on the Matrix Board. The circuit had a TTL driver (74ALS244) on its input, which fanned out the signal to both a Schmitt-trigger circuit $(7414+74$ S08), which limited the pulse width into the edge algorithm gates to $15-22$ $\mathrm{ns}$, and an inverter (74S04) to drive the block's inputs as a nearest-neighbor in other unit cells. The three edge-algorithm gates (74H21) each were used to switch on a coupled-emitter transistor (2N5770) circuit, which added a fixed current level to the Wire-OR (MV1,2 and 3) 


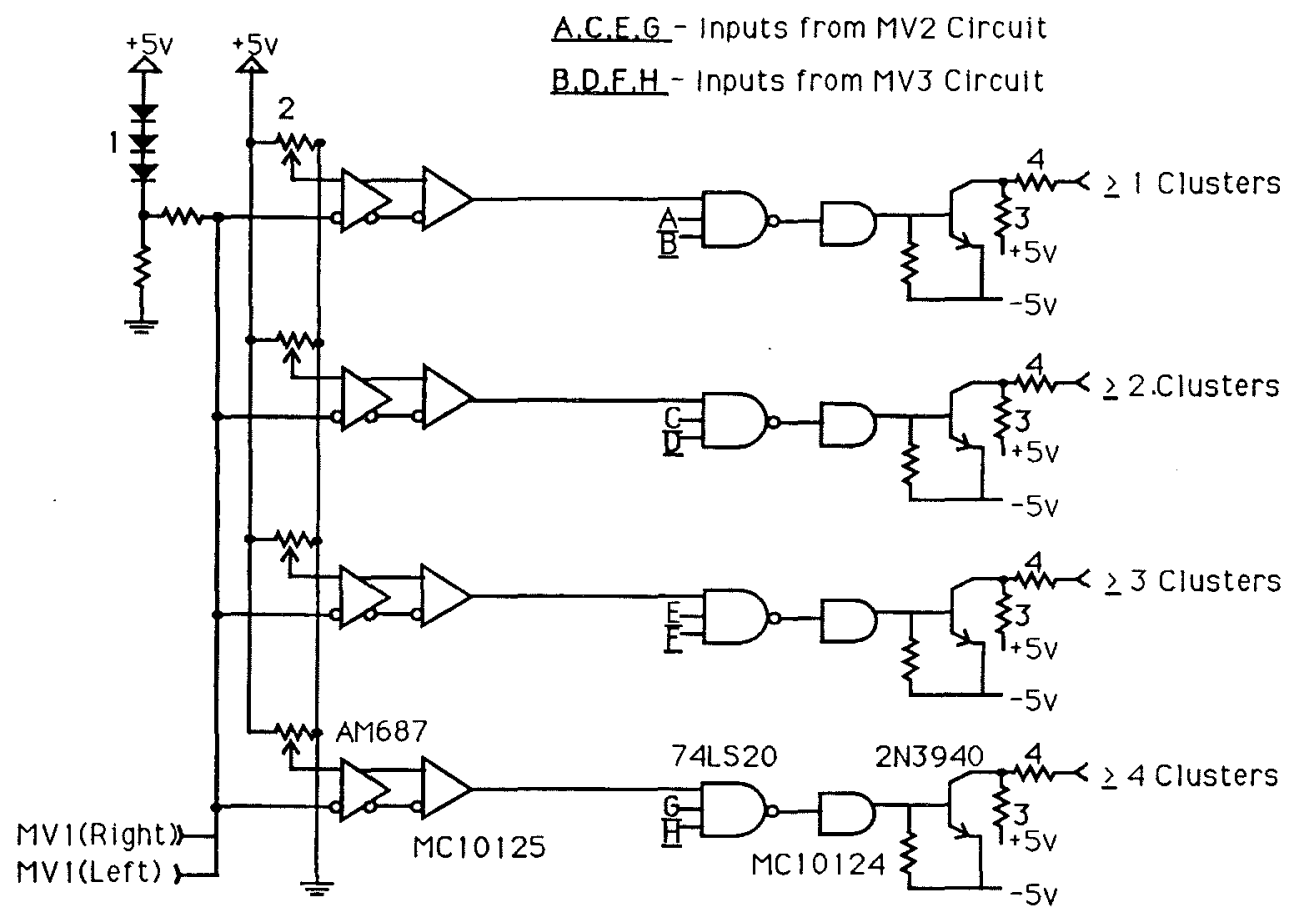

Note:

1 all diodes are IN914

2 2Kת-20 turn pot: sets AM687 threshold

- turn clockwise for lower threshold voltage

3 blas resistors are $\mathrm{IK} \Omega$

4 series output resistors are $10 \Omega$

Figure A.7: Schematic of Summing Output Board. Note that only the discrimination circuits for one of the edge-algorithms (MV1) is shown in the schematic. The circuits for MV2 and MV3 were identical to that shown for MV1. Their inputs to the final NAND gates (74LS20) are indicated in the note at the top of the diagram. 
MC10125), each with a threshold adjusted through a 20-turn $2 K \Omega$ potentiometer. The threshold of the first discriminator was adjusted so that its output went HIGH when the level of the summed inputs indicated $\geq 1$ cluster edge. The second discriminator was set for $\geq 2$ clusters, the third for $\geq 3$ and so on. The analog nature of the wireOR inputs meant that care had to be taken to make sure that the thresholds were set properly, and they were checked for each unit cell on the Matrix Boards.. The logical AND (74LS20/MC10124) of the three discriminated edge-algorithm outputs was used to make the final outputs of the trigger, as we required that only the minimum of the results of the three algorithms be used. A transistor stage (2N3940) provided NIM-level output signals.

The $\gamma$ trigger had a total delay between the input to the block signal discriminators and the final cluster-count outputs of $<70 \mathrm{~ns}$, which met the design requirement. While the interconnection between the circuits of the trigger was defined by the particular lead glass array configuration used, these circuits could be re-wired for use on other arrays. 


\section{References}

[1] S. Rudaz. Spin and polarization studies of weak decays. In K. Heller, editor, Proceedings of the 8th International Symposium on High Energy Physics, 1988.

[2] T. D. Lee and C. N. Yang. General partial wave analysis of the decay of a hyperon of spin $\frac{1}{2}$. Phys. Rev., 108:1645, 1957.

[3] Particle Data Group. Review of particle properties. Phys. Lett., 204B:1, 1988.

[4] O. E. Overseth and S. Pakvasa. Final-state interactions in nonleptonic hyperon decays. Phys. Rev., 184:1663, 1969.

[5] J. F. Donoghue. Overview of the theory of hyperon decays. In K. Heller, editor, Proceedings of the 8th International Symposium on High Energy Physics, 1988.

[6] B. W. Lee and A. Swift. Phys. Rev., 136:B228, 1964.

[7] J. Donoghue et. al. Low-energy weak interactions of quarks. Phys. Rep., 131:320, 1986.

[8] A. LeYaounc et. al. Nucl. Phys., B148:321, 1979.

[9] J. Pati and C.H. Woo. Phys. Rev., D3:2920, 1971.

[10] J. Finjord and M. K. Gaillard. Hyperon decays revisited. Phys. Rev., D22:778, 1980. 
[11] G. Nardulli. A fresh look at the pole model of weak non leptonic and radiative hyperon decays. Il Nuovo Cim., 100A:485, 1988.

[12] B. Stech H.G. Dosch, M. Lamin. Diquarks, qcd sum rules, and weak decays. 1988. HE-THEP-88-28 (to be published).

[13] Robert Handler et. al. Precise measurement of the asymmetry parameter in the decay $\Xi^{0} \rightarrow \Lambda \pi^{0}$. Physical Review $D, 25: 639,1982$.

[14] Rameika et. al. Phys. Rev., D33:3172, 1986.

[15] Zech et. al. Nucl. Phys., B124:413, 1977.

[16] P. T. Cox et. al. Phys. Rev. Lett., 46:877, 1981.

[17] Philip Scott Martin. Lambda-Proton Scattering at Fermilab. PhD thesis, University of Wisconsin-Madison, 1977. (unpublished).

[18] P. Skubic et. al. Phys. Rev., D18:3115, 1978.

[19] Peter MacDonald Border. CP-Violation in the Decay $K^{0} \rightarrow \pi^{+} \pi^{-} \pi^{0}$. PhD thesis, University of Michigan, 1988. (unpublished).

[20] Catherine Chase James. A Measurement of the Branching Ratio of the Weak Radiative Decay $\Xi^{0} \rightarrow \Lambda \gamma$. $\mathrm{PhD}$ thesis, University of Minnesota, 1987. (unpublished).

[21] A. Beretvas et. al. Phys. Rev., D34:53, 1986.

[22] V. L. Highland. Nucl. Instr. and Meth., 129:497, 1975. and Nucl. Instr. and Meth. $161,171,(1979)$.

[23] K. B. Luk et. al. Phys. Rev., D38:19, 1988.

[24] K. Heller et. al. Phys. Rev. Lett., 51:2025, 1983.

[25] G. Bunce et. al. Nucl. Instr. and Meth., 172:553, 1980. 
[26] Poulard et. al. Phys. Lett., 46B:135, 1973.

[27] Clayton et. al. Nucl. Phys., B95:130, 1975.

[28] Dauber et. al. Phys. Rev., 179:1262, 1969.

[29] Mayeur et. al. Nucl. Phys., B47:33, 1972. Errata Nucl. Phys. B53 268.

[30] Baltay et. al. Phys. Rev., D9:49, 1974.

[31] G. Bunce et. al. Phys. Rev. Lett., 36:1113, 1976. 